UTF 325, to appear in Physics Reports

\title{
Quantum Fields and Extended Objects in Space-Times with Constant Curvature Spatial Section
}

\author{
Andrei A. Bytsenko 出 \\ Department of Theoretical Physics, State Technical University \\ St. Petersburg 195251, Russia \\ Guido Cognolaf, Luciano Vanzo and Sergio Zerbinif \\ Dipartimento di Fisica, Università di Trento \\ and Istituto Nazionale di Fisica Nucleare, \\ Gruppo Collegato di Trento, Italia
}

\begin{abstract}
The heat-kernel expansion and $\zeta$-regularization techniques for quantum field theory and extended objects on curved space-times are reviewed. In particular, ultrastatic space-times with spatial section consisting in manifold with constant curvature are discussed in detail. Several mathematical results, relevant to physical applications are presented, including exact solutions of the heat-kernel equation, a simple exposition of hyperbolic geometry and an elementary derivation of the Selberg trace formula. With regards to the physical applications, the vacuum energy for scalar fields, the one-loop renormalization of a self-interacting scalar field theory on a hyperbolic space-time, with a discussion on the topological symmetry breaking, the finite temperature effects and the Bose-Einstein condensation, are considered. Some attempts to generalize the results to extended objects are also presented, including some remarks on path integral quantization, asymptotic properties of extended objects and a novel representation for the one-loop (super)string free energy.
\end{abstract}

PACS: 04.62.+v, 11.10.Wx, 11.25.-w

\footnotetext{
1 e-mail: root@fmf.stu.spb.su (subject: Prof. A.A. Bytsenko)

e-mail: cognola@science.unitn.it

${ }^{3}$ e-mail: vanzo@science.unitn.it

${ }^{4}$ e-mail: zerbini@science.unitn.it
} 


\section{Contents}

\begin{tabular}{llc}
\hline 1 & Introduction & 3
\end{tabular}

\begin{tabular}{|lll}
2 & Path integral and regularization techniques in curved space-times & 7
\end{tabular}

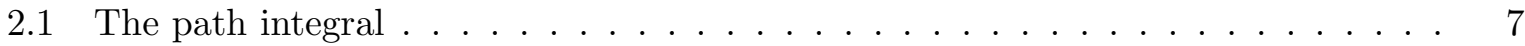

2.2 The $\zeta$-function regularization $\ldots \ldots \ldots \ldots \ldots \ldots$

$2.2 .1 \quad$ Complex powers and heat kernel of elliptic operators . . . . . . . . . . . 9

2.2 .2 The $\zeta$-function $\ldots \ldots \ldots \ldots \ldots \ldots$

2.3 Other regularization techniques $\ldots \ldots \ldots \ldots \ldots \ldots \ldots$

2.4 The one-loop effective action and the renormalization group equations . . . . . . 16

$2.5 \quad$ Static and ultrastatic space-time $\ldots \ldots \ldots \ldots \ldots \ldots \ldots$

2.6 Finite temperature effects $\ldots \ldots \ldots \ldots \ldots \ldots \ldots \ldots$

2.6 .1 The free energy $\ldots \ldots \ldots \ldots \ldots \ldots \ldots \ldots$

2.6 .2 The thermodynamic potential . . . . . . . . . . . . . . . 21

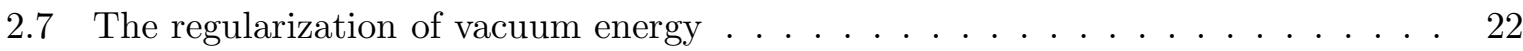

\begin{tabular}{|lll}
\hline & Constant curvature manifolds & 25
\end{tabular}

3.1 The heat kernel and $\zeta$-function on the torus . . . . . . . . . . . . . . 25

3.2 Representations and recurrence relations for $\zeta$-function on the sphere . . . . . . . 26

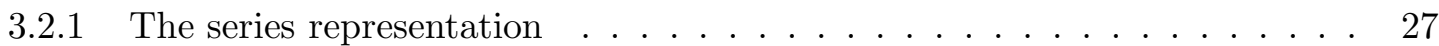

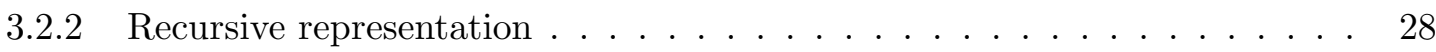

$3.2 .3 \quad$ A complex integral representation . . . . . . . . . . . . . . . 28

3.3 Hyperbolic manifolds . . . . . . . . . . . . . . . . . . . . . . . . . . . . 29

3.3 .1 The Laplace operator and the density of states . . . . . . . . . . . . . . . 32

3.3 .2 The Dirac operator and the density of states $\ldots \ldots \ldots$. . . . . . . . 35

3.4 Compact hyperbolic manifolds $\ldots \ldots \ldots \ldots \ldots \ldots \ldots$

3.4 .1 The Selberg trace formula for scalar fields . . . . . . . . . . . . . . . . 39

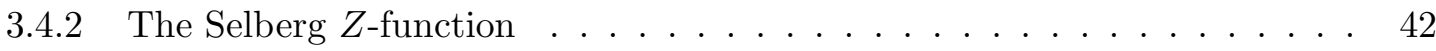

$3.4 .3 \quad$ The Selberg trace formula for compact $H^{3} / \Gamma \ldots \ldots$. . . . . . . . . . . 43

3.4.4 The Selberg trace formula for compact $H^{2} / \Gamma \ldots \ldots$. . . . . . . . . . . 46

$3.4 .5 \quad$ Recurrence relations for heat kernel and $\zeta$-function . . . . . . . . . . . . 47

$3.4 .6 \quad$ A factorization formula and zero modes . . . . . . . . . . . . . . . 48

4 Zero temperature quantum properties on ultrastatic manifolds with constant $\begin{array}{ll}\text { curvature section } & 51\end{array}$

4.1 The vacuum energy for massive scalar fields on $\mathbb{R} \times T^{N} \ldots \ldots \ldots$. . . . . . . . 51

$4.2 \quad$ The vacuum energy for massive scalar fields on $\mathbb{R} \times S^{N} \ldots \ldots \ldots \ldots$. . . . . . 53

4.3 The vacuum energy for massive scalar fields on $\mathbb{R} \times H^{N} / \Gamma$. . . . . . . . . . . . 53

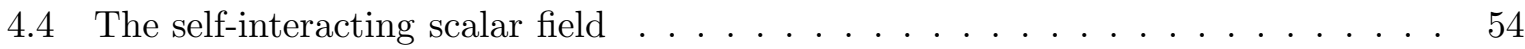

4.4 .1 Renormalization conditions. . . . . . . . . . . . . . . . . 56

$4.4 .2 \quad$ The ultrastatic space-time $\mathbb{R} \times H^{3} / \Gamma \ldots \ldots \ldots \ldots \ldots \ldots$

$4.4 .3 \quad$ The ultrastatic space-times $\mathbb{R} \times T^{3}, \mathbb{R} \times S^{3}, \mathbb{R}^{2} \times S^{2}, \mathbb{R}^{2} \times H^{2} / \Gamma$. . . 60

\begin{tabular}{|lll}
5 & Quantum $p$-branes in curved space-times & $\mathbf{6 1}$
\end{tabular}

$5.1 \quad$ Classical properties of (super) $p$-branes . . . . . . . . . . . . . . . . . 61

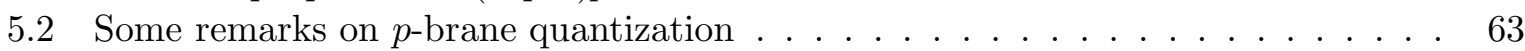

5.3 Classification of 3-geometries $\ldots \ldots \ldots \ldots \ldots \ldots \ldots$

5.4 Classification of 4 -geometries $\ldots \ldots \ldots \ldots \ldots \ldots$

5.5 The path integral associated with loop expansion $\ldots \ldots \ldots \ldots$. . . . . . . 70

5.5 .1 The free relativistic point particle $\ldots \ldots \ldots \ldots$. . . . . . . 70 
5.5 .2 The $p$-brane model . . . . . . . . . . . . . . . . . 70

5.6 The Casimir energy for $p$-branes in space-times with constant curvature $\ldots$. . . 72

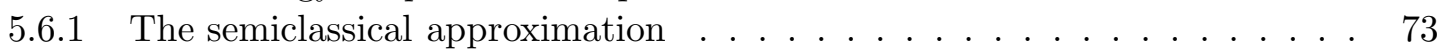

5.6 .2 The static potential on toroidal spaces . . . . . . . . . . . . . . . 74

\begin{tabular}{|lll}
\hline & Asymptotic properties of $p$-brane quantum state density & $\mathbf{7 6}$
\end{tabular}

6.1 Asymptotic properties of generating functions . . . . . . . . . . . . . 76

6.2 Asymptotic density of $p$-brane quantum states $\ldots \ldots \ldots \ldots$. . . . . . . 78

6.3 Asymptotic density of parabosonic string quantum states . . . . . . . . . . . 80

6.4 Extented objects and black holes $\ldots \ldots \ldots \ldots \ldots$. . . . . . . . . . 82

7 Finite temperature quantum properties on ultrastatic space-time with hyper$\begin{array}{ll}\text { bolic spatial section } & 85\end{array}$

7.1 The free energy and thermodynamic potential . . . . . . . . . . . . . . . . . . 85

7.2 The Bose-Einstein condensation on $\mathbb{R} \times H^{3} \ldots \ldots \ldots$. . . . . . . . . . . . . . . 89

7.3 The finite temperature effective potential for a self-interacting scalar field on

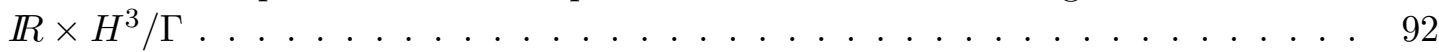

\begin{tabular}{|lll}
\hline & Strings at finite temperature & 95
\end{tabular}

8.1 The Mellin-Barnes representation for one-loop string free energy $\ldots . \ldots$

$8.2 \quad$ High genus contributions to string free energy $\ldots \ldots \ldots \ldots$. . . . . . . . . 98

\begin{tabular}{ll}
\hline Appendices & 102
\end{tabular}

\begin{tabular}{|lll}
\hline A Admissible regularization functions for the determinant & 102
\end{tabular}

B The heat kernel on a Riemannian manifold without boundary $\quad 104$

B.1 Spectral coefficients for a Laplace-like operator . . . . . . . . . . . . . . . . . 104

B.2 Heat kernel exact solutions on constant curvature manifolds . . . . . . . . . . . . . 105

C The explicit computation of $\zeta$-function on compact manifolds without bound$\begin{array}{ll}\text { ary } & 107\end{array}$

\begin{tabular}{ll}
\hline D Useful relations & 109
\end{tabular}

\begin{tabular}{ll}
\hline References & 111
\end{tabular} 


\section{Introduction}

The problem of reconciling general relativity with quantum theory, which has been deeply studied in the early twentieth century, has not yet found a consistent and satisfactory solution. The theory of quantum fields in curved space-time deals with quantum matter field in an external gravitational field and may be considered as a preliminary step toward the complete quantum theory of gravity (see for example Refs. 11, 2, 3]).

Electromagnetic, weak and strong interactions may be unified in the so called grand unified theory and this may be achieved in the well established framework of relativistic quantum field theory. Roughly speaking, this framework deals with point objects (particles) and their related description in terms of local quantum fields. However, it is well known that the gravitational interaction, which classically is well described by general relativity, cannot be consistently described within this framework (non perturbative renormalizability). For this reason, the interest for extended objects has been grown.

The most simple extended object is the (super)-string [4]. An interesting feature of string is that its energy-mass spectrum contains a massless spin-two particle, which may be interpreted as a graviton. Moreover, a fundamental length (Regge slope) appears in a natural way and so the ultraviolet problems seem to be cured. When the fundamental length goes to zero the usual field theory can be recovered. The consequence of these facts leads to the hope that the second quantized version of string theory will provide a consistent picture of all elementary interactions at quantum level (see for example Ref. [4]).

A further insight for the construction of a consistent quantum theory of all known elementary interactions has come with the proposal of the modern Kaluza-Klein theories, which may provide a unified fundamental theory in 4-dimensions by starting from an underlying theory in higher dimensions. Some years ago these ideas were used in the framework of field theory, including gravitation and extended objects, that is (super)strings and (super)p-branes (generalization of strings), which at the moment are receiving a great interest not only among physicists of fundamental particles, but also among cosmologists.

In this report, we shall maily concentrate on space-times admitting a constant curvature spatial section. The main motivation for studying fields and (super)strings (at zero and finite temperature) on ultrastatic space-times in which the spatial section is a manifold with constant curvature, stems from the fact that, for a fixed value of the cosmological time, such manifolds describe locally spatially homogeneous isotropic universes.

More recently, there have been attempts to investigate also the role of topology in the physics of quantum fields and extended objects and the phenomenon of topological mass generation and dynamical symmetry breaking have been discovered. The non trivial topological space-times which mainly have been considered till now, are the ones with the torus and sphere geometries. In these cases, the spectrum of the relevant operators is generally known. So far, this fact has been fruitfully used in all the investigations. It has to be noted however, that within the space-time with constant curvature spatial sections, the compact hyperbolic manifolds have also to be considered. The distinguish features of them are the huge number of possible geometries and the highly non trivial topologies with respect to the previous ones and for this reason they may provide many interesting alternative solutions for the construction of models of our universe. In these cases the spectrum of the relevant operators is, in general, not explicitly known. However, there exists a powerful mathematical tool, the Selberg trace formula [5], which permits to evaluate some interesting physically global quantities, like the vacuum energy or the one-loop effective action. Unfortunately, for a compact hyperbolic manifold, it is difficult to get an explicit and manageable form for local quantities like the propagators, which are explicitly known for the torus, sphere and non compact hyperbolic geometries (see for example [6]).

As we have already mentioned, in the last decades there has been a great deal of investigations 
on the properties of interacting quantum field theories in a curved space-time (see Ref. [7]). Several techniques have been employed, among these we would like to mention the backgroundfield method [1] within path-integral approach, which is very useful in dealing with the oneloop approximation and which permits the one-loop effective action to be evaluated. As a consequence, all physical interesting quantities can be, in principle, evaluated. Throughout the paper, we mainly shall make use of the path-integral quantization.

The (Euclidean) one-loop effective action, as derived from the path-integral, is a ill defined quantity, being related to the determinant of the fluctuation operator, which is a second order elliptic non negative differential operator. Since it is not a bounded operator, its real eigenvalues $\lambda_{n}$ diverge for large $n$. Hence, the naive definition of the determinant leads to a formally divergent quantity. It turns out that these divergences are controlled by geometrical quantities, which are referred to as Seeley-DeWitt or spectral coefficients. In order to control the divergences and extract the finite part, many regularizations have been proposed and used. One of the most promising, which works very well also in a curved manifold, is the so called "zetafunction regularization" (see [8]), which gives a rigorous definition of the determinant of the fluctuation operator and therefore of the regularized one-loop effective action. This latter has to be renormalized, since it contains an arbitrary normalization parameter, coming from the path integral measure. Here, other regularizations will be analyzed and their close relation with the $\zeta$-function one will be pointed out.

It should be stressed that, on a generic Riemannian manifold, the $\zeta$-function related to the Laplace-Beltrami operator is generally unknown, but nevertheless, some physical interesting quantities can be related to its Mellin inverse transform, which has a computable asymptotic expansion (heat kernel expansion). The coefficients of this asymptotic expansion are the ones which control the ultraviolet one-loop divergences (see for example [1, 9]. Thus, they are suitable only in describing local properties (ultraviolet behaviour), but some important physical issues, like phase transitions in cosmological models, quantum anomalies and symmetry breaking due to changes in topology, are insensitive to them.

The situation is really better on space-time with constant curvature spatial sections, where the effective action can be explicitly constructed, because the spectrum is explicitly known (zero and positive curvature) or by making use of Selberg trace formula (compact hyperbolic manifolds).

The zeta-function regularization technique is also useful in the study of quantum fields or strings at finite temperature. It provides complex integral representations (Mellin-Barnes) for one-loop thermodynamic quantities, from which low and high temperature expansions can be directly obtained.

With regards to the mathematical results we shall employ, for the reader's convenience, part of the paper (mainly Secs. 2 and 3) is devoted to the summary of some useful techniques, with particular attention to the physical applications. We derive the main features of $\zeta$-function and its relation with the heat kernel expansion and report the results we need in several Appendices. In particular, for torus $T^{N}$, sphere $S^{N}$ and hyperbolic space $H^{N}$, we give heat kernel and $\zeta$-function for the Laplace-Beltrami operator in closed form. We briefly review the Minakshisundaram-Pleijel-Schwinger-Seeley-DeWitt methods which permit to obtain the heat parametrix for a differential operator on a compact manifold without boundary (see, for example, the review article [9] and the monography [2]. With regard to this, important results on a partly summed form of the heat-kernel expansion have been presented in Refs. [10, 11, 12].

After having discussed some properties of the hyperbolic space $H^{N}$, the Harish-Chandra measure associated with scalar and spinor fields, is derived solving the Laplace and Dirac equations on $H^{N}$ and evaluating explicitly the corresponding density of states. We also present a brief survey of the geometry and topology of compact hyperbolic manifolds of the form $H^{N} / \Gamma$, $\Gamma$ being group of isometries of $H^{N}$ (see for example [13]). An elementary and self-contained dis- 
cussion of Selberg trace formula for the strictly hyperbolic manifold $H^{N} / \Gamma$ and the properties of the Selberg $Z$-function and its logarithmic derivative are also presented. Here, our aim is merely didactic and we refer to the original mathematical literature for a more rigorous exposition. In particular, for the two cases $H^{2} / \Gamma$ and $H^{3} / \Gamma$, which are potentially interesting for a physical viewpoint, we also include elliptic elements in the group $\Gamma$. In this way we shall deal more properly with orbifold.

As for as the physical applications are concerned, making use of the $\zeta$-function regularization, we derive the expressions of vacuum and free energies (finite temperature effects within the canonical and grandcanonical ensemble) for scalar and spinor fields, on arbitrary $D$-dimensional ultrastatic Riemannian manifolds. The relation of these results with the more general static case is pointed out, if does not exist any Killing horizon in the manifold. Making use of the Selberg trace formula, such expressions are specialized to compact manifolds of the form $\mathbb{R} \times H^{2} / \Gamma$ and $\mathbb{R} \times H^{3} / \Gamma$ or, more generally, on Kaluza-Klein space-times with the topology $\mathbb{R}^{D-p} \times$ $H^{p} / \Gamma$. It is found that, for trivial line bundles, one can obtain a negative contribution to the vacuum energy. On such manifolds with compact hyperbolic spatial section, we also compute the effective potential. In the special but important case of $\mathbb{R} \times H^{3} / \Gamma$, we carry on the oneloop renormalization program for the $\lambda \phi^{4}$ self-interacting field theory. The role of topology is analyzed in this context and the possibility of mass generation, symmetry breaking or restoration is discussed.

The finite temperature effects are studied by employing a complex integral representation for the free energy, which will be referred to as Mellin-Barnes representation. We show how it is particular useful in deriving the high temperature expansion.

The inclusion in the previous theories of the chemical potential is quite straightforward and this permits the grand canonical potential and all related thermodynamic quantities to be computed. In particular, we give high and low temperature expansions for the thermodynamic potential on $\mathbb{R} \times H^{3}$ and we discuss in detail the problem of the Bose-Einstein condensation for a relativistic ideal gas. The critical temperature is evaluated and its dependence on curvature is exhibited.

Some attempts are made in order to generalize these results to the extended objects. We discuss a semiclassical approximation to path-integral quantization for the bosonic sector, when a closed $p$-brane sweeps out on a $D=(p+1)$-dimensional compact hyperbolic manifold. Within the same approximation, we present a general formula of the static potential for $p$-branes compactified on Kaluza-Klein space-times of the form $T^{q} \times \Pi_{\alpha} S^{N_{\alpha}} \times \Pi_{\beta}\left(H^{2} / \Gamma_{\beta}\right) \times \mathbb{R}^{D-p}$.

It is shown that the quantum behaviour of the potential and its extrema depend on the choice of twists.

Starting from the semiclassical quantization of $p$-branes (torus compactification leads to discrete mass spectrum) and making use of some rigorous results of number theory (the theorem of Meinardus) we evaluate the asymptotic form of the density of states. In particular, the explicit form of the prefactors of total level degeneracy is derived. The universal feature of the density, which grows slower than

the one for black holes, but faster than that for strings, leads to the breakdown on the canonical description for $p>1$. With this regard, we also comment on the possible connection between black holes and $p$-branes.

As far as the finite temperature effects for extended objects are concerned, due to the asymptotic behaviour of the state level density, we have argued above that only the $p=1$ (string) seems to be consistently tractable within the canonical ensemble. For this reason, by using the analogous of the Mellin-Barnes representation for one-loop thermodynamic quantities, we exhibit a novel representation for the free energy of open and closed bosonic strings and open superstrings, in terms of a Laurent series. It is shown that in this formalism, the Hagedorn temperatures arise as the radius of convergence of the corresponding Laurent series (convergence 
condition). The high genus (interacting) string case is also discussed and the independence of the Hagedorn temperature on the genus is pointed out.

\section{Notations.}

- Throughout the paper we shall use units in which the speed of light, Planck and Boltzmann constants have the values $c=\hbar=k=1$.

- $K_{\nu}(z)$ are the Mc Donald functions, $\Gamma(s)$ is the Euler gamma function, $\zeta_{R, H}$ are the RiemannHurwitz zeta-functions, $Z_{\mathcal{R}}(z, \vec{g}, \vec{h})$ is the Epstein $Z$-function, $Z(s)$ and $\Xi(s)$ are the Selberg functions.

- $K_{n}(A)$ and $\zeta(s \mid A)$ are the spectral coefficients and the $\zeta$-function respectively, related to an elliptic operator $A$.

- By $\mathcal{M}^{\mathcal{N}}$ we shall indicate a $N$ dimensional Riemannian manifold, with Euclidean metric $g_{i j}$, $g$ being its determinant $(i, j=1, \ldots, N)$. $A$ or $L_{N}$ are generic differential operators and $\Delta_{N}$ is the Laplace-Beltrami operator.

- In the physical applications, we shall deal with space-times $\mathcal{M}^{\mathcal{D}}$ with metric $g_{\mu \nu},(\mu, \nu=$ $0, \ldots, D-1)$ and with spatial section $\mathcal{M}^{\mathcal{N}}, N=D-1$.

- We shall mainly deal with manifolds $\mathcal{M}^{\mathcal{N}}$ with constant curvature $\kappa$. Then we shall set $\mathrm{E}_{N}=\Delta_{N}+\alpha^{2}+\kappa \varrho_{N}^{2}$, where $\varrho_{N}=(N-1) / 2$ and $\alpha$ is an arbitrary constant. 


\section{Path integral and regularization techniques in curved space- times}

Path-integral quantization [14 is a very powerful approach to the quantization of a generic physical system. By means of it, it is possible to investigate, in an elegant and economic way, several issues including for example gauge theory [15], finite temperature effects [16], quantum anomalies [17], effective action [18] and quantum gravity [19, 20].

In the present section we discuss in some detail the quantization, via path integral, of matter systems living on curved background manifolds. With regard to this, the evaluation of the propagator and heat-kernel in curved space-time by a path integral was presented in Ref. [21], where the Einstein universe (having constant curvature spatial section) was solved exactly.

Since in the external field or in the Gaussian approximation of the full theory, the oneloop effective action can be formally expressed by means of determinants of elliptic differential operators, one needs a regularization scheme in order to give a rigorous meaning to such determinants. For this purpose, $\zeta$-function regularization [22, 23, 8, 13], as we have already anticipated, is very convenient and it shall be extensively used throughout the paper. Some properties and representations will be derived in Sec. 2.2.2. For the sake of completeness, other (equivalent) regularizations of operator determinants will be discussed in Sec. 2.3.

In this Section, the one-loop effective potential is introduced and, for a $\lambda \phi^{4}$ theory, the renormalization group equations are derived. The problems concerning the regularization of physical quantities like the vacuum energy are analyzed in some detail. Zero temperature as well as finite temperature effects are considered and partition function, free energy and thermodynamic potential, for which we give some useful representations, are investigated.

\subsection{The path integral}

To start with, let $\phi$ a matter quantum field living on a given curved background $D$ dimensional manifold with metric $g_{\mu \nu}$. Its physical properties are conveniently described by means of the path integral functional (in the following, an infinite normalization constant we will be systematically neglected)

$$
Z[g]=\int d[\phi] e^{i S[\phi, g]},
$$

where $S[\phi, g]$ is the classical action and the functional integral is taken over all matter fields satisfying suitable boundary or periodicity conditions. By the generic argument $g$ we leave understood the functional dependence of the external background field.

We recall that when the space-time is asymptotically flat and the integral is over the fields infinitesimally closed to the classical vacuum at infinity, $Z[g]$ is supposed to give the vacuumto-vacuum amplitude [24]. Feymann boundary conditions are implicitly assumed in the action functional. The related functional $W[g]=-i \ln Z[g]$ plays also un important role, since it generates the effective field equations, namely the classical ones plus quantum corrections. For a non-asymptotically flat space-time, the physical meaning of the functional $Z[g]$ as vacuum persistence amplitude is less clear, but the functional $W[g]$ is still supposed to describe the effective action. Of particular interest is the case when a complex metric (manifold) can be found such that there exist both a Lorentzian and an Euclidean real sections. In this case, one can relate the functional $Z[g]$ to the Euclidean "partition function"

$$
Z_{E}[g]=\int d[\phi] e^{-S_{E}[\phi, g]}
$$

for which the methods of the theory of the elliptic operators can be applied [8]. It should be noted that, in the general case, this is not an unique procedure. However, for a static metric, 
it is always possible to find the associated Euclidean section by simply looking at the form of the metric tensor. Thus, in general, it could not be possible to define consistently the partition function. A sufficient condition is to restrict the analisys to the so called "strongly elliptic metrics", namely complex metrics for which a function $f$ exists such that $\operatorname{Re}\left(f g_{\mu \nu}\right)$ is positive definite. In this case, the path integral is well defined [8].

Throughout the paper we shall dealing with matter field in a external gravitational field or we shall make use of the one-loop approximation. In the latter case, since the dominant contributions to $Z_{E}[g]$ will come from fields which are near the classical solution $\phi_{c}$, which extremizes the action, for our purposes, it will be sufficient to consider classical actions which are quadratic in the quantum fluctuations $\tilde{\phi}=\phi-\phi_{c}$. In fact, a functional Taylor expansion of the action around $\phi_{c}$ gives

$$
S[\phi, g]=S_{c}\left[\phi_{c}, g\right]+\left.\frac{\delta^{2} S[\phi, g]}{\delta \phi^{2}}\right|_{\phi=\phi_{c}} \frac{\tilde{\phi}^{2}}{2}+\text { higher order terms in } \tilde{\phi},
$$

$S_{c}\left[\phi_{c}, g\right]$ being the classical action and so the one-loop approximated theory is determined by the "zero temperature partition function"

$$
Z\left[\phi_{c}, g\right] \sim \exp \left(-S_{c}\left[\phi_{c}, g\right]\right) \int d[\tilde{\phi}] \exp \left(-\frac{1}{2} \int \tilde{\phi} A \tilde{\phi} d^{D} x\right)
$$

To ensure diffeomorphism invariance of the functional measure, the functional integral dummy variables $\tilde{\phi}$ are chosen to be scalar densities of weight $-1 / 2$ [17] (see also Ref. [25]). In Eq. (2.3) the analogue of the Wick rotation of the time axis in the complex plane has to be understood. In this manner, the metric $g_{\mu \nu}$ becomes Euclidean and the small disturbance operator $A$ selfadjoint and non-negative. For non self-interacting fields minimally coupled to gravity, $A$ is equal to the field operator, that is the Klein-Gordon operator for scalar fields and the Dirac operator for fermion fields.

With these assumptions, the partition function can be formally computed in terms of the real eigenvalues $\lambda_{n}$ of the operator $A$. This can be easily done by observing that the functional measure $d[\tilde{\phi}]$ reads

$$
d[\tilde{\phi}]=\prod_{n} \frac{d c_{n}}{\sqrt{2 \pi \ell}}
$$

$c_{n}$ being the expansion coefficients of $\tilde{\phi}$ in terms of the eigenvectors of $A$ and $\ell$ an arbitrary parameter necessary to adjust dimensions. It will be fixed by renormalization. In this manner, for neutral scalar fields one has [8]

$$
Z^{(1)}=\prod_{n} \frac{1}{\sqrt{2 \pi \ell}} \int_{-\infty}^{\infty} d c_{n} e^{-\frac{1}{2} \lambda_{n} c_{n}^{2}}=\left[\operatorname{det}\left(A \ell^{2}\right)\right]^{-1 / 2} .
$$

The functional $Z^{(1)}\left[\phi_{c}, g\right]$ determines the one-loop quantum corrections to the classical theory. An expression very similar to the latter is valid for charged scalar fields and also for anticommuting spinor fields. To consider all the cases, we may write

$$
Z^{(1)}=\left[\operatorname{det}\left(A \ell^{2}\right)\right]^{-\nu}, \quad \ln Z^{(1)}=-\nu \ln \operatorname{det}\left(A \ell^{2}\right),
$$

where $\nu=-1,1,1 / 2$ according to whether one is dealing with Dirac spinor, charged or neutral scalar fields respectively. Eq. (2.6) is valid also for a multiplet of matter fields. In such a case $A$ is a matrix of differential operators. 


\subsection{The $\zeta$-function regularization}

Looking at equations above, we see that in order to define the (one-loop) quantum theory via path integral, one needs a suitable regularization of determinant of (elliptic) differential operator, which is formally infinity, since the naive definition of the product over the eigenvalues gives rise to badly divergent quantity. This stems from the fact that the number $\mathcal{N}(\lambda)$ of eingenvalues whose value is less than $\lambda$ admits an asymptotic expansion of the form [26, 27, 19, 28]

$$
\mathcal{N}(\lambda) \simeq \sum_{n<N} \frac{K_{n}}{\Gamma\left(\frac{N-n}{2}\right)+1} \lambda^{\frac{N-n}{2}}, \quad \lambda \rightarrow \infty,
$$

where $K_{n}$ are the integrated spectral coefficients we are going to introduce in the next subsection. As one can see, they control the degree of divergence of the determinant. Throughout the paper, we shall mainly make the choice of $\zeta$-function regularization. In Sec. 2.3 we shall also discuss a class of other possible and equivalent regularizations. We recall that $\zeta(s \mid A)$ is defined in terms of the complex power of the operator $A$ or, via a Mellin transform, by the trace of the heat kernel related to $A$.

\subsubsection{Complex powers and heat kernel of elliptic operators}

Here we just remind the definition of complex power of an elliptic operator as given by Seeley in Ref. 29] and its link with the heat kernel. We briefly discuss some properties of it, which we need in the following, referring the interested reader to the literature for more details.

Let $A$ be an invertible elliptic differential (or pseudo-differential) operator of positive order $p$, defined on a $\mathrm{N}$-dimensional compact manifold $\mathcal{M}^{N}$, with boundary or without boundary. Then, for $\operatorname{Re} s<0$, one can define a semigroup $A^{s}$ by

$$
A^{s}=-\frac{1}{2 \pi i} \int_{\Gamma} \frac{z^{s}}{A-z} d z,
$$

and this semigroup can be extended to a group containing $A$ and the identity. The curve $\Gamma$ goes from $\infty$ to $\infty$ and around the origin along a small circle, enclosing the spectrum of $A$. If $A^{s}$ is a pseudo-differential operator of order $p \cdot s$ the Seeley method permits to build up a parametrix for its kernel $A\left(x, x^{\prime} ; s\right)$, which is a continuous function for $\operatorname{Re}(p \cdot s)<-N$.

The Seeley method for the construction of the parametrix of $A^{s}$ and as a consequence, a related asymptotic expansion for $\operatorname{det} A$, is very general in principle, but quite complicated with regard to the computational point of view. Several alternative approaches to the problem have been proposed, but mostly associated with a related quantity. In fact the kernel of $A^{s}$ can be expressed, by means of a Mellin transform, as a function of the heat kernel $K_{t}\left(x, x^{\prime} \mid A\right)=$ $e^{-t A}\left(x, x^{\prime}\right)$. That is

$$
A^{-s}\left(x, x^{\prime}\right)=\frac{1}{\Gamma(s)} \int_{0}^{\infty} t^{(s-1)}\left[K_{t}\left(x, x^{\prime} \mid A\right)-P_{0}\left(x, x^{\prime} \mid A\right)\right] d t,
$$

$K_{t}\left(x, x^{\prime} \mid A\right)$ being a bi-spinor or bi-tensor density of weight $-1 / 2$ and $P_{0}$ the projector onto zero modes. The presence of zero modes does not create particular problems. Here we continue to suppose the operator $A$ to be invertible, then zero modes are absent and the projector $P_{0}$ can be ignored. When necessary zero modes shall be carefully considered.

$K_{t}\left(x, x^{\prime}\right)$ satisfies the heat-type equation (the argument $A$ is understood)

$$
\partial_{t} K_{t}\left(x, x^{\prime}\right)+A K_{t}\left(x, x^{\prime}\right)=0, \quad \quad \lim _{t \rightarrow 0_{+}} K_{t}\left(x, x^{\prime}\right)=[g(x)]^{1 / 4} \delta\left(x, x^{\prime}\right)\left[g\left(x^{\prime}\right)\right]^{1 / 4},
$$

the solution of which admits an asymptotic expansion of the kind (see for example Ref. [30])

$$
K_{t}(x, x) \simeq \frac{\sqrt{g(x)}}{(4 \pi)^{N / p}} \sum_{0}^{\infty} k_{n}(x) t^{(n-N) / p} .
$$


This expansion is also valid when the manifold has a boundary. Thus $K_{t}\left(x, x^{\prime}\right)$, together Eq. (2.9), must satisfy suitable boundary conditions.

A useful property of the heat kernel is factorization on a product space, that is

$$
K_{t}^{\mathcal{M}_{1} \times \mathcal{M}_{2}}\left(\left(x_{1}, x_{2}\right),\left(x_{1}^{\prime}, x_{2}^{\prime}\right) \mid A\right)=K_{t}^{\mathcal{M}_{1}}\left(x_{1}, x_{1}^{\prime} \mid A_{1}\right) K_{t}^{\mathcal{M}_{2}}\left(x_{2}, x_{2}^{\prime} \mid A_{2}\right),
$$

where the operators $A_{1}$ and $A_{2}$ are defined on $\mathcal{M}_{1}$ and $\mathcal{M}_{2}$ respectively and $A=A_{1}+A_{2}$. Eq. (2.11) is equivalent to the fact that $\operatorname{Tr} e^{-t\left(A_{1}+A_{2}\right)}=\operatorname{Tr} e^{-t A_{1}} \operatorname{Tr} e^{-t A_{2}}$ when the operators $A_{1}$ and $A_{2}$ commute.

On a manifold without boundary, all $k_{n}(x)$ coefficients with odd $n$ are vanishing and so Eq. (2.10) assumes a simpler form. In the present work we shall essentially deal with second order differential operators $(p=2)$ on manifolds without boundary $\left(k_{2 n+1}=0\right)$. Putting $k_{2 n}(x)=a_{n}(x)$ one gets [31, 32]

$$
K_{t}(x, x) \simeq \frac{\sqrt{g(x)}}{(4 \pi t)^{N / 2}} \sum_{0}^{\infty} a_{n}(x) t^{n},
$$

the expansion coefficients $a_{n}(x)$ being local invariant matrices depending on curvature and torsion tensors and their covariant derivatives. They all, but $a_{0}$ vanish in the flat-free case, while $a_{0}$ is always equal to the identity matrix.

We have been mentioned (see also Ref. [1]) that these coefficients control the one-loop divergences of the effective action and of the related quantities, like the stress energy momentum tensor. Hence, the explicit knowledge of the $a_{n}$ is important in the physical applications. In general, a closed form for the heat kernel is unknown also for simple operators like the Laplacian and for this reason the related asymptotic expansion has been extensively investigated . Many methods for computing the $K_{n}$ on smooth Riemannian manifolds have been proposed by physicists and mathematicians and some coefficients have been computed for Riemannian manifolds with and without boundary as well as for the case of Riemann-Cartan manifolds.

Here we would like just to mention the general procedure related to Seeley method [33, 34], and the (well known to physicists) Schwinger [35] and DeWitt 32 technique, which work very well for manifolds without boundary. When the manifold has a boundary the computation of the spectral coefficients is a much more difficult task than the boundaryless case [36, 30]. The literature on this subject is very vast and we refer the reader to Ref. [37] (and references therein), where some spectral coefficients in the case of manifolds with boundary have been computed by a very powerful method on any smooth Riemannian manifold. For a general derivation of heat coefficients on a Riemann-Cartan manifold see Ref. [38]. Extension of previous methods to manifolds with boundary can be found in Refs. [39, 40] and references cited therein.

\subsubsection{The $\zeta$-function}

Let $A$ be an invertible operator with the properties of Sec. 2.2.1. The (generalized Riemann) $\zeta$-function related to $A$, first investigated in Ref. [41], can be defined by

$$
\zeta(s \mid A)=\operatorname{Tr} A^{-s}=\int_{\mathcal{M}} A^{-s}(x, x) d^{N} x=\frac{1}{\Gamma(s)} \int_{0}^{\infty} t^{s-1} \operatorname{Tr} e^{-t A} d t
$$

where, in writing the last term (the Mellin transform of the heat kernel), we have made use of Eq. (2.8) disregarding zero modes. When $x \neq x^{\prime}, A^{-s}\left(x, x^{\prime}\right)$ is an entire function of $s$, while $A^{-s}(x, x)$ is a meromorphic function having simple poles on the real axis. Positions and number of poles depend on the order of operator and dimension of $\mathcal{M}$. Then we see that according to definition (2.13), also $\zeta(s \mid A)$ is a meromorphic function with simple poles on the real axis. In particular, $\zeta(s \mid A)$ is analytic at $s=0$. When the operator $A$ admits a complete set of 
eigenvectors with eigenvalues $\lambda_{n}$, then Eq. (2.13) is the analytic continuation of the definition (valid for Re $s$ sufficiently large)

$$
\zeta(s \mid A)=\sum_{n} \lambda_{n}^{-s}
$$

from which

$$
\zeta^{\prime}(s \mid A)=-\sum_{n} \lambda_{n}^{-s} \ln \lambda_{n} .
$$

When $A$ is not an invertible operator, the null eigenvalues must be omitted in Eq. (2.14).

From definition (2.14), we easily obtain the scaling property

$$
\zeta\left(s \mid A \ell^{2}\right)=\ell^{-2 s} \zeta(s \mid A),
$$

valid for any $\ell$, while from Eqs. (2.15) and (2.16), we see that a natural definition of determinant of $A$, in the sense of analytic continuation, is given by means of formula [8]

$$
\ln \operatorname{det}\left(A \ell^{2}\right)=-\zeta^{\prime}(0 \mid A)+\zeta(0 \mid A) \ln \ell^{2} .
$$

The above equation shows that $\zeta(0 \mid A)$ governs the scale dependence of the quantity $\ln \operatorname{det}\left(A \ell^{2}\right)$.

The residues of the poles of $\zeta$-function are directly related to heat kernel coefficients. Integrating the heat expansion, we get a parametrix for $K(t \mid A)=\operatorname{Tr} e^{-t A}$ of the kind (here we limit our analisys to a second order differential operator, namely $p=2$ )

$$
K(t \mid A) \simeq \sum_{n=0}^{\infty} K_{n}(A) t^{(n-N) / 2}
$$

where $K_{n}(A)$ are the (integrated) spectral coefficients, that is

$$
K_{n}(A)=\frac{1}{(4 \pi)^{N / 2}} \int_{\mathcal{M}} k_{n}(x \mid A) \sqrt{g(x)} d x .
$$

In order to derive the meromorphic structure of $\zeta(s \mid A)$, the standard procedure is to split the integration over $t$ of Eq. (2.13) in two parts, $(0,1)$ and $(1, \infty) . K(t \mid A)$ is a smooth function of $t$ for $t \rightarrow \infty$ and so the integral from 1 to $\infty$ in Eq. (2.8) is an entire function of $s$. The rest of the integral can be explicitly computed using expansion (2.18), which is valid for small $t$. Thus, for a second order differential operator, we get

$$
\begin{aligned}
\zeta(s \mid A) & \simeq \frac{1}{\Gamma(s)}\left[\sum_{n=0}^{\infty} K_{n}(A) \int_{0}^{1} t^{s-(N-n) / 2-1} d t+\int_{1}^{\infty} t^{s-1} K(t \mid A) d t\right] \\
& =\sum_{n=0}^{\infty} \frac{K_{n}(A)}{\Gamma(s)\left(s-\frac{N-n}{2}\right)}+\frac{\hat{G}(s \mid A)}{\Gamma(s)}=\frac{K_{N}(A)}{\Gamma(s+1)}+\frac{G(s \mid A)}{\Gamma(s)},
\end{aligned}
$$

where

$$
\hat{G}(s \mid A)=\int_{1}^{\infty} t^{s-1} K(t \mid A) d t
$$

is an entire function of $s$ while $G(s \mid A)$ has poles at all the points $s=(N-n) / 2$, but $s=0$. In fact $G(0 \mid A)$ is the principal part of $\Gamma(s) \zeta(s \mid A)$ at $s=0$. On the contrary, due to the presence of $\Gamma(s)$ in Eq. 2.20), not all the points $s=(N-n) / 2$ are simple poles of $\zeta(s \mid A)$. Moreover, in the absence of boundaries, all $K_{n}$ with odd $n$ vanishes, so in this case, for even $\mathrm{N}$ we have $\mathrm{N} / 2$ poles situated at the integers $n=1,2, \ldots, N / 2$ with residues $K_{N-2 n} / \Gamma(n)$ while for odd $\mathrm{N}$ we have infinite poles at all half integers $n \leq N / 2$ with residues $K_{N-2 n} / \Gamma(n)$. In particular, $\zeta(s \mid A)$ is finite for $s=0$ and one has the useful relations

$$
\begin{aligned}
& \zeta(0 \mid A)=K_{N}(A), \\
& \zeta^{\prime}(0 \mid A)=\gamma K_{N}(A)+G(0 \mid A)=-\ln \operatorname{det} A .
\end{aligned}
$$


Zero-modes. As we have already mentioned before, zero modes must be omitted in the definition of $\zeta$-function. Now we briefly describe a possible way to proceed in order to define the determinant of the operator $A$ when $\mathcal{N}$ zero-modes are present. First of all we introduce the positive operator $A_{\lambda}=A+\lambda(\lambda>0)$ and define the regularized determinant of $A$ by means of equation

$$
\begin{aligned}
\ln \operatorname{det} A & =-\lim _{\lambda \rightarrow 0}\left[\zeta^{\prime}\left(0 \mid A_{\lambda}\right)+\mathcal{N} \ln \lambda\right] \\
& =-\gamma K_{N}(A)-\lim _{\lambda \rightarrow 0}\left[G\left(0 \mid A_{\lambda}\right)+\mathcal{N} \ln \lambda\right] .
\end{aligned}
$$

In this way all zero-modes are automatically subtracted. In writing the latter equation we have used Eq. (2.22) and the fact that the logarithmic divergence for $\lambda \rightarrow 0$ comes from $G\left(A_{\lambda}\right)$ (actually from $\hat{G}\left(0 \mid A_{\lambda}\right)$ ). In fact, it is easy to see looking at Eq. (2.21) that $\hat{G}\left(0 \mid A_{\lambda}\right) \sim-\mathcal{N} \ln \lambda$ plus a regular function in the limit $\lambda \rightarrow 0$.

The next step is to observe that the function $\exp \left(-G\left(0 \mid A_{\lambda}\right)\right.$ has a zero of order $\mathcal{N}$ in $\lambda=0$. Thus, deriving Eq. (2.23) $\mathcal{N}$ times with respect to $\lambda$ and taking the limit $\lambda \rightarrow 0$ we finally get

$$
\operatorname{det} A=\frac{e^{-\gamma K_{N}(A)}}{\mathcal{N} !} \lim _{\lambda \rightarrow 0} \frac{d^{\mathcal{N}}}{d \lambda^{\mathcal{N}}} \exp \left[-G\left(0 \mid A_{\lambda}\right)\right] .
$$

The factorization property. We consider two positive elliptic operators $A_{1}$ and $A_{2}$ acting on functions in $\mathcal{M}^{\mathcal{N}_{\infty}}$ and $\mathcal{M}^{\mathcal{N} \in}\left(N_{1} \leq N_{2}\right)$. According to the factorization property, Eq. (2.11), the heat kernel related to the operator $A=A_{1}+A_{2}$ is the product of the two kernels related to the operators $A_{1}$ and $A_{2}$ or, what is the same,

$$
K(t \mid A)=K\left(t \mid A_{1}\right) K\left(t \mid A_{2}\right) .
$$

Using such a relation for the trace of the heat kernel now we obtain an interesting representation for $\zeta(s \mid A)$ in terms of $\zeta\left(s \mid A_{1}\right)$ and $\zeta\left(s \mid A_{2}\right)$. Of course, when $\mathcal{M}_{\infty}$ or/and $\mathcal{M}_{\in}$ are non-compact, we have to consider $\zeta$-function densities.

We start with Mellin representation of $\zeta$-function, Eq. (2.13), that is

$$
\zeta(s \mid A)=\frac{1}{\Gamma(s)} \int_{0}^{\infty} t^{s-1} K\left(t \mid A_{1}\right) K\left(t \mid A_{2}\right) d t .
$$

The right hand side of the latter equation can be transformed in an integral in the complex plane using Mellin-Parseval identity, Eq. (D.2). To this aim we choose $f(t)=K\left(t \mid A_{1}\right) t^{s / 2}$, $g(t)=K\left(t \mid A_{2}\right) t^{s / 2-1}$ and rewrite Eq. (2.24) by means of Eq. (D.2). In this way we obtain what we call the Mellin-Barnes representation for the $\zeta$-function

$$
\zeta(s \mid A)=\frac{1}{2 \pi i \Gamma(s)} \int_{\operatorname{Re} z=c} \Gamma\left(\frac{s}{2}+z\right) \zeta\left(\frac{s}{2}+z \mid A_{1}\right) \Gamma\left(\frac{s}{2}-z\right) \zeta\left(\frac{s}{2}-z \mid A_{2}\right),
$$

where $c$ is a real number in the common strip in which $\hat{f}(z)$ and $\hat{g}(1-z)$ are analytical (see Appendix $\mathrm{D}$ ). In our case this corresponds to the condition $-\frac{s-N_{1}}{2}<c<\frac{s-N_{2}}{2}$. Such a condition is restrictive but, as we shall see in the following, it can be relaxed in the applications of physical interest.

The generalization of the latter formula to the case in which $A_{1}$ or/and $A_{2}$ have also zeromodes can be derived by the same technique. It reads

$$
\begin{aligned}
\zeta(s \mid A)= & \mathcal{N}_{1} \zeta\left(s \mid A_{1}\right)+\mathcal{N}_{2} \zeta\left(s \mid A_{2}\right) \\
& +\frac{1}{2 \pi i \Gamma(s)} \int_{\operatorname{Re} z=c} \Gamma\left(\frac{s}{2}+z\right) \zeta\left(\frac{s}{2}+z \mid A_{1}\right) \Gamma\left(\frac{s}{2}-z\right) \zeta\left(\frac{s}{2}-z \mid A_{2}\right) d z,
\end{aligned}
$$

$\mathcal{N}_{1}$ and $\mathcal{N}_{2}$ being the number of zero-modes of $A_{1}$ and $A_{2}$ respectively. As usual, all zero-modes have to be omitted in the evaluation of $\zeta$-functions. 
Example: $S^{1} \times \mathcal{M}^{\mathcal{N}}$. It is of particular interest for physical applications (finite temperature quantum field theories), the case $\mathcal{M}^{\mathcal{D}}=\mathcal{S}^{\infty} \times \mathcal{M}^{\mathcal{N}}$, i.e. the manifold is the product between a circle $S^{1}$ and a (compact) $N$-dimensional manifold $\mathcal{M}^{\mathcal{N}}$. Then $A=-\partial_{\tau}^{2}+L_{N}$, where $\tau$ runs on $S^{1}$ and $L_{N}$ is an operator in $\mathcal{M}^{\mathcal{N}}$. If we assume that the field to satisfy periodic conditions (only for illustrative purposes) on $S^{1}$, then the eigenvalues of $A$ have the form

$$
\lambda_{n, j}=\left(\frac{2 \pi n}{\beta}\right)^{2}+\omega_{j}^{2}, \quad n=0, \pm 1, \pm 2, \ldots,
$$

$\beta$ (the inverse of the temperature $T$ ) being the circumference of $S^{1}$ and $\omega_{j}^{2}$ the eigenvalues of $L_{N}$. In this case we have

$$
\begin{aligned}
\zeta(s \mid A) & =\sum_{n=-\infty}^{\infty} \zeta\left(s \mid L_{N}+[2 \pi n / \beta]^{2}\right) \\
& =\frac{\beta \Gamma\left(s-\frac{1}{2}\right)}{\sqrt{4 \pi} \Gamma(s)} \zeta\left(s-\frac{1}{2} \mid L_{N}\right)+\frac{\beta}{\pi} \sum_{n=1}^{\infty} \int_{-\infty}^{\infty} e^{i n \beta t} \zeta\left(s \mid L_{N}+t^{2}\right) d t \\
& =\frac{\beta \Gamma\left(s-\frac{1}{2}\right)}{\sqrt{4 \pi} \Gamma(s)} \zeta\left(s-\frac{1}{2} \mid L_{N}\right)+\frac{\beta}{\sqrt{\pi} \Gamma(s)} \sum_{n=1}^{\infty} \int_{0}^{\infty} t^{s-3 / 2} e^{-n^{2} \beta^{2} / 4 t} K\left(t \mid L_{N}\right) d t .
\end{aligned}
$$

Eq. (2.28) have been derived from Eq. (2.27) using the Poisson summation formula, Eq. (D.1), while Eq. (2.29) have been directly obtained by using the Mellin representation (2.13) for $\zeta$ function in Eq. (2.28).

Using Eq. (2.25) we could obtain a complex integral representation of $\zeta$-function for this particular case, but we prefer to derive it directly from Eq. (2.29), since in this case the restriction on $c$ is not so stringent. This representation will be particularly useful for studying the high temperature expansion. To this aim we choose $f(t)=t^{s-3 / 2} K\left(t \mid L_{N}\right), g(t)=e^{-n^{2} \beta^{2} / 4 t}$ and use Eq. (2.29) and Mellin-Parseval identity, Eq. (D.2). As a result

$$
\begin{aligned}
\zeta(s \mid A)= & \frac{\beta \Gamma\left(s-\frac{1}{2}\right)}{\sqrt{4 \pi} \Gamma(s)} \zeta\left(s-\frac{1}{2} \mid L_{N}\right)+\frac{1}{\sqrt{\pi} \Gamma(s)} \frac{1}{2 \pi i} \times \\
& \int_{\operatorname{Re} z=c} \zeta_{R}(z) \Gamma\left(\frac{z}{2}\right) \Gamma\left(\frac{z-1}{2}+s\right) \zeta\left(\frac{z-1}{2}+s \mid L_{N}\right)\left(\frac{\beta}{2}\right)^{-(z-1)} d z,
\end{aligned}
$$

where $\zeta_{R}$ represents the usual Riemann $\zeta_{R}$-function and $c>N+1$.

From Eqs. (2.20), (2.28), (2.29) and (2.30) we get three representations for the derivative of $\zeta$, namely

$$
\begin{aligned}
\zeta^{\prime}(0 \mid A) & =-\beta \zeta^{(r)}\left(-\frac{1}{2} \mid L_{N}\right)+\frac{\beta}{\pi} \sum_{n=1}^{\infty} \int_{-\infty}^{\infty} e^{i n \beta t} \zeta^{\prime}\left(0 \mid L_{N}+t^{2}\right) d t \\
& =-\beta \zeta^{(r)}\left(-\frac{1}{2} \mid L_{N}\right)+\frac{\beta}{\sqrt{\pi}} \sum_{n=1}^{\infty} \int_{0}^{\infty} t^{-3 / 2} e^{-n^{2} \beta^{2} / 4 t} K\left(t \mid L_{N}\right) d t \\
& =-\beta \zeta^{(r)}\left(-\frac{1}{2} \mid L_{N}\right)+\frac{\beta}{\pi i} \int_{\operatorname{Re} z=c} \zeta_{R}(z) \Gamma(z-1) \zeta\left(\frac{z-1}{2} \mid L_{N}\right) \beta^{-z} d z,
\end{aligned}
$$

where we have introduced the definition

$$
\zeta^{(r)}\left(-\frac{1}{2} \mid L_{N}\right)=\operatorname{PP} \zeta\left(-\frac{1}{2} \mid L_{N}\right)+(2-2 \ln 2) \operatorname{Res} \zeta\left(-\frac{1}{2} \mid L_{N}\right),
$$

$\mathrm{PP}$ and Res being respectively the finite part and the residue of the function at the specified point. Note that the residue of $\zeta\left(s \mid L_{N}\right)$ at $s=-1 / 2$ is equal to $-K_{N+1}\left(L_{N}\right) / \sqrt{4 \pi}, K_{N+1}\left(L_{N}\right)$ being the coefficient of $\sqrt{t}$ in the asymptotic expansion of $K\left(t \mid L_{N}\right)$. Thus, when this coefficient 
is vanishing, there is no pole at $s=-1 / 2$ and the $\mathrm{PP}$ prescription gives the value of $\zeta\left(s \mid L_{N}\right)$ at the same point.

For use later, we define, for any constant $\ell$

$$
\zeta^{(r)}\left(s \mid L_{N} \ell^{2}\right)=\operatorname{PP} \zeta\left(s \mid L_{N}\right)+(2-2 \ln 2 \ell) \operatorname{Res} \zeta\left(s \mid L_{N}\right) .
$$

Example: $\mathbb{R}^{p} \times \mathcal{M}^{\mathcal{N}}$. In many physical problems (for example in Kaluza-Klein theories), the manifold $\mathcal{M}$ is the product of the flat $p$-dimensional manifold $\mathbb{R}^{p}$ and a compact $N$-dimensional manifold $\mathcal{M}^{\mathcal{N}}$ and the differential operator of interest takes the form $A=-\Delta_{p}+L_{N}$, where $\Delta_{p}$ is the Laplace operator acting on functions in $\mathbb{R}^{p}$ and $L_{N}$ is an operator acting on functions in $\mathcal{M}^{\mathcal{N}}$. In this case, using Eq. (2.24), we obtain the useful relation

$$
\begin{aligned}
\frac{\zeta(s \mid A)}{\Omega_{p}} & =\frac{1}{(4 \pi)^{\frac{p}{2}} \Gamma(s)} \int_{0}^{\infty} t^{s-\frac{p}{2}-1} K\left(t \mid L_{N}\right) d t \\
& =\frac{\Gamma\left(s-\frac{p}{2}\right)}{(4 \pi)^{\frac{p}{2}} \Gamma(s)} \zeta\left(s-\frac{p}{2} \mid L_{N}\right),
\end{aligned}
$$

where $\Omega_{p}$ is a large volume in $\mathbb{R}^{p}$. The left hand side of Eq. 2.35) then represents a density in $\mathbb{R}^{p}$.

In the particular case in which $p=1$, we write down the derivative at $s=0$ of the latter equation. Using Eq. (2.16) we easily obtain

$$
\frac{\ln \operatorname{det} A}{\Omega_{1}}=-\frac{\zeta^{\prime}(0 \mid A)}{\Omega_{1}}=\zeta^{(r)}\left(-1 / 2 \mid L_{N}\right)
$$

\subsection{Other regularization techniques}

Here we shall discuss a class of regularizations based on the Schwinger representation (see for example [42]), which contains $\zeta$-function regularization as particular case. In order to work with dimensionless quantities, we put $B=A \ell^{2}$. The regularized determinant of the operator $B$ may be defined by

$$
(\ln \operatorname{det} B)_{\varepsilon}=-\int_{0}^{\infty} t^{-1} \varrho(\varepsilon, t) \operatorname{Tr} e^{-t B} d t,
$$

where $\varrho(\varepsilon, t)$ is a suitable regularization function of the dimensionless parameter $t$, which has to satisfy the two requirements we are now going to discuss. First, for fixed $t>0$, the limit as $\varepsilon$ goes to zero must be equal to one. Second, for fixed and sufficiently large $\varepsilon, \varrho(\varepsilon, t)$ has to regularize the singularity at $t=0$ coming from the heat kernel expansion (2.18) related to $B$. The analytic continuation will be used to reach small values of $\varepsilon$. As we shall see in the following, these requirements do not uniquely determine the regularization function $\varrho$.

Using Eq. (2.18) in Eq. (2.37) one can easily see that the number of divergent terms for $\varepsilon \rightarrow 0$ is equal to $Q+1, Q$ being the integer part of $D / 2$. They are proportional to the spectral coefficients $K_{0}, \ldots K_{Q}$ (which contain the full dependence on the geometry), the prefactors depending on the regularization function 42]. In Appendix A, this general result is explicitly verified in several examples.

Regularizations in $\mathbb{R}^{D}$. In the rest of this Section, in the particular but important case $A=-\Delta_{D}+M^{2}$ in $\mathbb{R}^{D}$, we would like to show that the finite part of (ln $\left.\operatorname{det} B\right)_{\varepsilon}$ (that is the effective potential, see following Sec. 2.4) is uniquely determined, modulo a constant which can be absorbed by the arbitrary scale parameter $\ell$ and can be evaluated without making use of the explicit knowledge of the regularization function. As usual, in $\mathbb{R}^{D}$ we consider a large region $\mathcal{R}$ of volume $\Omega_{\mathcal{R}}$ and the limit $\Omega_{\mathcal{R}} \rightarrow \infty$ shall be taken at the end of calculations. It has to be 
stressed that, apart from a topological contribution, this calculation is already enough to give the one loop-effective potential in a constant curvature space-time, i.e. the identity contribution.

From the known results on $\mathbb{R}^{D}$ (see Eq. $(\overline{B .13})$ ) we have

$$
\frac{\operatorname{Tr} e^{-t B}}{\Omega_{\mathcal{R}}}=\frac{e^{-t \ell^{2} M^{2}}}{\left(4 \pi t \ell^{2}\right)^{D / 2}}
$$

where $M^{2}$ is a positive constant. As a consequence, from Eq. (2.37), we obtain the regularized expression

$$
\frac{(\ln \operatorname{det} B)_{\varepsilon}}{2 \Omega_{\mathcal{R}}}=-\frac{1}{2}\left(\frac{1}{4 \pi \ell^{2}}\right)^{D / 2} \int_{0}^{\infty} t^{-(1+D / 2)} \varrho(\varepsilon, t) e^{-t \ell^{2} M^{2}} d t \equiv f_{D}(\varepsilon, M) .
$$

Now it is convenient to distinguish between even $(D=2 Q)$ and odd $(D=2 Q+1)$ dimensions. In order to derive a more explicit form of the divergent terms for $\varepsilon \rightarrow 0$, let us consider the $Q^{\text {th }}$-derivative of $f(\varepsilon, M)$ with respect to $M^{2}$. One gets

$$
\frac{d^{Q} f_{D}(\varepsilon, M)}{d M^{2 Q}}=\frac{(-1)^{Q}}{2}\left(\frac{1}{4 \pi \ell^{2}}\right)^{D / 2} B_{e, o}(\varepsilon, M)
$$

where

$$
\begin{aligned}
& B_{e}(\varepsilon, M)=-\int_{0}^{\infty} t^{-1} \varrho(\varepsilon, t) e^{-t M^{2}} d t=\ln M^{2}+b+c_{Q}(\varepsilon)+O(\varepsilon), \\
& B_{o}(\varepsilon, M)=-\int_{0}^{\infty} t^{-3 / 2} \varrho(\varepsilon, t) e^{-t M^{2}} d t=2 M \sqrt{\pi}+b+c_{Q}(\varepsilon)+O(\varepsilon) .
\end{aligned}
$$

In the derivation of the above equations we have made use of the properties of the regularization functions. Furthermore, in Eqs. (2.41) and (2.42) $b$ is a constant and $c_{Q}(\varepsilon)$ is a function of $\varepsilon$, but not of $M$. We have used the same symbols for even and odd dimensions, but of course they represent different quantities in the two cases.

Making use of Eqs. (2.41) and (2.42) in Eq. (2.40) a simple integration gives

$$
\begin{aligned}
& f_{2 Q}(\varepsilon, M)=\frac{(-1)^{Q}}{2 Q !(4 \pi)^{Q}}\left[\ln \left(\ell^{2} M^{2}\right)-C_{Q}+b\right] M^{D}+\ell^{-D} \sum_{n=0}^{Q} c_{n}(\varepsilon)(\ell M)^{2 n}+O(\varepsilon), \\
& f_{2 Q+1}(\varepsilon, M)=\frac{(-1)^{Q} \Gamma(-Q-1 / 2)}{2(4 \pi)^{Q+1 / 2}} M^{D}+\ell^{-D} \sum_{n=0}^{Q} c_{n}(\varepsilon)(\ell M)^{2 n}+O(\varepsilon),
\end{aligned}
$$

where we have set $C_{Q}=\sum_{n=1}^{Q} \frac{1}{n}$.

The dimensionless integration constants $c_{n}(\varepsilon)$, which are divergent for $\varepsilon \rightarrow 0$, define the counterterms which must be introduced in order to remove the divergences. For the physical interesting case $D=4$, one gets

$$
\frac{(\ln \operatorname{det} B)_{\varepsilon}}{2 \Omega_{\mathcal{R}}}=\frac{M^{4}}{64 \pi^{2}}\left[\ln \left(\ell^{2} M^{2}\right)-\frac{3}{2}+b\right]+c_{2}(\varepsilon) f^{4}+c_{1}(\varepsilon) M^{2} \ell^{-2}+c_{0}(\varepsilon) \ell^{-4},
$$

in agreement with the well known result obtained in Refs. [43, 44], where some specific regularizations were used for $D=4$.

Some remarks are in order. The constants $b$ and $c_{n}(\varepsilon)$ depend on the choice of the regularization function and on the Seeley-Dewitt coefficient $a_{n}(x, B)$, but $b$ can be absorbed by the arbitrary scale parameter $\ell$. As a result, the finite part of $(\ln \operatorname{det} B)_{\varepsilon}$ (the effective potential) does not depend on the regularization, as expected. In Appendix A several examples of admissible regularizations of the kind we have discussed are reported as an illustration of the above general result. In particular we show that $\zeta$-function, as well as other known regularizations, can be derived from Eq. (2.37) with a suitable choice of $\varrho(\varepsilon)$.

In the rest of the paper, we shall make use of the $\zeta$-function regularization. 


\subsection{The one-loop effective action and the renormalization group equations}

In this subsection we assume $A$ to be an elliptic second order differential operator acting on fields in $\mathcal{M}^{\mathcal{D}}$ and depending on the classical solution $\phi_{c}$. It will be of the form $A=-\Delta_{D}+V^{\prime \prime}\left(\phi_{c}, g\right)$ (the prime indicates the derivative with respect to $\phi), V(\phi, g)$ being a scalar function describing the self-interaction of the field, the coupling with gravity and containing furthermore local expressions of dimension $D$ involving curvature tensors and non-quadratic terms in the field. These latter terms have, in general, to be included in order to ensure the renormalizability of the theory [45]. When $V(\phi, g)$ is almost quadratic in the matter fields, then $A$ is just the classical field operator.

The one loop effective action takes the form [18]

$$
\begin{aligned}
\Gamma\left[\phi_{c}, g\right] & =S_{c}\left[\phi_{c}, g\right]+\frac{1}{2} \ln \operatorname{det}\left(A \ell^{2}\right) \\
& =\int\left[V_{e f f}\left(\phi_{c}, g\right)+\frac{1}{2} Z\left(\phi_{c}, g\right) g^{i j} \partial_{i} \phi_{c} \partial_{j} \phi_{c}+\cdots\right] \sqrt{g} d^{D} x .
\end{aligned}
$$

Eq. (2.46) defines the one-loop effective potential $V_{e f f}\left(\phi_{c}\right)$. It is given by

$$
V_{e f f}\left(\phi_{c}, g\right)=V_{c}\left(\phi_{c}, g\right)+V^{(1)}\left(\phi_{c}, g\right)=V\left(\phi_{c}, g\right)+\frac{\ln \operatorname{det}\left(A \ell^{2}\right)}{2 \Omega(\mathcal{M})},
$$

$\Omega(\mathcal{M})$ being the volume of $\mathcal{M}$. The quantum corrections $V^{(1)}\left(\phi_{c}, g\right)$ to the classical potential $V\left(\phi_{c}, g\right)$ are of order $\hbar$ and are formally divergent.

Renormalization group equations. For the sake of completeness, now we derive the renormalization group equations for the $\lambda \phi^{4}$ theory [46] on a 4-dimensional compact smooth manifold without boundary, the extension to manifolds with boundary being quite straightforward. The derivation which we present is valid only at one-loop level. For a more general discussion see for example Ref. [47, 48] and references cited therein.

We regularize the one-loop effective action $(2.46)$ by means of $\zeta$-function. In this way we have

$$
\Gamma\left[\phi_{c}, g\right]=S_{c}\left[\phi_{c}, g\right]-\frac{1}{2} \zeta^{\prime}\left(0 \mid A \ell^{2}\right) .
$$

The more general classical action has the form

$$
\begin{aligned}
& S_{c}(\eta)=\int\left[\Lambda-\frac{\phi_{c} \Delta \phi_{c}}{2}+\right.\left.\frac{\lambda \phi_{c}^{4}}{24}+\frac{m^{2} \phi_{c}^{2}}{2}+\frac{\xi R \phi_{c}^{2}}{2}\right] \sqrt{g} d^{4} x \\
&+\int\left[\varepsilon_{1} R+\frac{\varepsilon_{2} R^{2}}{2}+\varepsilon_{3} W\right] \sqrt{g} d^{4} x,
\end{aligned}
$$

$W=C^{i j r s} C_{i j r s}$ being the square of the Weyl tensor and $\eta=\eta_{q} \equiv\left(\Lambda, \lambda, m^{2}, \xi, \varepsilon_{1}, \varepsilon_{2}, \varepsilon_{3}\right)(q=$ $0, \ldots, 6)$ the collection of all coupling constants. We disregard the Gauss-Bonnet invariant since its integral over the manifold is proportional to the Euler-Poincare characteristic, namely, it is both a topological invariant as well as scale independent. As a consequence it does not affect the scale dependence of the effective action and we can dispense with it.

Now we consider a conformal transformation $\tilde{g}_{\mu \nu}=\exp (2 \sigma) g_{\mu \nu}$ with $\sigma$ a constant (scaling). By the conformal transformation properties of the fields, one can easily check that $\tilde{S}_{c}(\eta)=S_{c}(\tilde{\eta})$, where $\tilde{\eta}$ are all equal to $\eta$ except $\Lambda, m^{2}$, and $\varepsilon_{1}$. For these, we have

$$
\tilde{\Lambda}=\Lambda e^{4 \sigma}, \quad \tilde{m}^{2}=m^{2} e^{2 \sigma}, \quad \tilde{\varepsilon}_{1}=\varepsilon_{1} e^{2 \sigma} .
$$


In the same way, for the eigenvalues $\tilde{\alpha}_{n}(\eta)$ of the small disturbance operator $\tilde{A}(\eta)$ we have $\tilde{\alpha}_{n}(\eta)=e^{-2 \sigma} \alpha_{n}(\tilde{\eta})$. From this transformation rule for the eigenvalues, we immediately get the transformations for $\zeta\left(s \mid A \ell^{2}\right)$ and $\zeta^{\prime}\left(s \mid A \ell^{2}\right)$. They read

$$
\begin{aligned}
& \zeta\left(s \mid \tilde{A} \ell^{2}\right)=e^{2 s \sigma} \zeta\left(s \mid A(\tilde{\eta}) \ell^{2}\right), \\
& \zeta^{\prime}\left(s \mid \tilde{A} \ell^{2}\right)=e^{2 s \sigma}\left[\zeta^{\prime}\left(s \mid A(\tilde{\eta}) \ell^{2}\right)+2 \sigma \zeta\left(s \mid A\left(\tilde{\eta} \ell^{2}\right)\right]\right.
\end{aligned}
$$

and finally

$$
\tilde{\Gamma}(\eta)=\Gamma(\tilde{\eta})-\sigma \zeta(0 \mid A(\tilde{\eta})) .
$$

We have seen in Sec. 2.2.2 that in $N$ dimensions $\zeta(0 \mid A)$ is related to the spectral coefficient $k_{N}(x \mid A)$ by means of the equation

$$
\zeta(0 \mid A)=\frac{1}{(4 \pi)^{N / 2}} \int k_{N}(x \mid A) \sqrt{g} d^{N} x .
$$

What is relevant for our case is $k_{4}(x)=a_{2}(x \mid A(\tilde{\eta}))$ which is well known to be (see Eq. (B.2))

$$
\begin{aligned}
a_{2}(x \mid A(\tilde{\eta}))= & -\frac{\lambda \Delta \phi_{c}^{2}}{12}+\frac{\lambda \tilde{m}^{2} \phi_{c}^{2}}{2}+\frac{\lambda(\xi-1 / 6) R \phi_{c}^{2}}{2}+\frac{\lambda^{2} \phi_{c}^{4}}{8}+\frac{\tilde{m}^{4}}{2} \\
& +\tilde{m}^{2}\left(\xi-\frac{1}{6}\right) R+\frac{(\xi-1 / 6)^{2} R^{2}}{2}+\frac{W}{120}-\frac{G}{360}+\frac{(5-\xi) \Delta R}{6},
\end{aligned}
$$

where $G=R^{i j r s} R_{i j r s}-4 R^{i j} R_{i j}+R^{2}$ is the Gauss-Bonnet invariant. Hence, integrating $a_{2}$ over the manifold and disregarding total divergences, one finally gets

$$
\tilde{\Gamma}(\eta)=S_{c}(\tilde{\eta}(\sigma))-\frac{1}{2} \zeta^{\prime}(0 \mid \tilde{A})=\Gamma(\tilde{\eta}(\sigma))+O\left(\hbar^{2}\right)
$$

The new parameters $\eta(\sigma)$ are related to the old ones $\eta=\eta(0)$ by

$$
\begin{array}{lr}
\Lambda(\sigma)-\Lambda=\frac{m^{4}}{2} \hat{\sigma}, & \lambda(\sigma)-\lambda=3 \lambda^{2} \hat{\sigma}, \\
m^{2}(\sigma)-m^{2}=\lambda m^{2} \hat{\sigma}, & \xi(\sigma)-\xi=\left(\xi-\frac{1}{6}\right) \lambda \hat{\sigma}, \\
\varepsilon_{1}(\sigma)-\varepsilon_{1}=\left(\xi-\frac{1}{6}\right) m^{2} \hat{\sigma}, & \varepsilon_{2}(\sigma)-\varepsilon_{2}=\left(\xi-\frac{1}{6}\right)^{2} \hat{\sigma}, \\
\varepsilon_{3}(\sigma)-\varepsilon_{3}=\frac{1}{30} \hat{\sigma} . &
\end{array}
$$

Here we have set $\hat{\sigma}=-\sigma / 16 \pi^{2}$. With the substitution $\sigma \rightarrow-2 \tau$, the latter equations have been given in Refs. [49, 50]. These formulae tell us that all parameters (coupling constants) develop, as a result of quantum effects, a scale dependence, even if classically they are dimensionless parameters. This means that in the quantum case, we have to define the coupling constants at some particular scale. Differentiating Eq. (2.53) with respect to $\sigma$, one immediately gets the renormalization group equations [49, 50]. 


\subsection{Static and ultrastatic space-times}

We have seen that the renormalization procedure, in general requires a bare cosmological constant in the gravitational action. Thus one has to consider solutions of Einstein equations with a non vanishing cosmological constant. Among the globally static solutions of Einstein equations in vacuum, besides the Minkowski space-time with $\Lambda=0$, de Sitter $(\Lambda>0)$ and anti-de Sitter $(\Lambda<0)$ space-times are particularly interesting. The first one is simply connected, while the second one has a simple connected universal covering (see for example Ref. [51]). These manifolds are also maximally symmetric and consequently they have constant curvature. One may also consider other static space-times by quotienting with a discrete group of isometries, but then one can encounter pathologies, like the existence of closed time-like geodesics. It is an interesting fact that the Euclidean sections corresponding to Minkowski, de Sitter and anti-de Sitter space-time are the three constant curvature space forms $\mathbb{R}^{4}, S^{4}$ and $H^{4}$ respectively. As a consequence, the Euclidean field theory on these manifolds can have some relevance. However, in this subsection, we shall deal with an arbitrary and globally static space-time and we shall briefly discuss how it is possible, making use of conformal transformation techniques, to restrict ourselves to ultrastatic space-times.

By definition, an ultrastatic space-time admits a globally defined coordinate system in which the components of the metric tensor are time independent and the condition $g_{00}=1$ and $g_{0 i}=0$ hold true. This means that the metric admits a global time-like orthogonal Killing vector field. In all physical applications of the present paper, we shall deal with an ultrastatic space-time $\mathcal{M}^{\mathcal{D}}=\mathbb{R} \times \mathcal{M}^{\mathcal{N}}(D=N+1)$. This is not a true restriction, since any static metric may be transformed in an ultrastatic one (optical metric) by means of a conformal transformation [52]. This fact permits us to compute all physical quantities in an ultrastatic manifold and, at the end of calculations, transform back them to a static one, with an arbitrary $g_{00}$. This has been done, for example, in Refs. [53, 54, 55, 56, 57, 58, 59] and in Ref. [60], where the existence of boundaries has also been taken into account. In particular, the approximation for the heatkernel on a static Einstein space-time was first introduced in Ref. [54], where the approximate propagator has been obtained in closed form by making use of the results contained in Ref. [21]. Here we would like to review the techniques of those papers.

To start with we consider a scalar field $\phi$ on a $D=N+1$-dimensional static space-time defined by the metric

$$
d s^{2}=g_{00}(\vec{x})\left(d x^{0}\right)^{2}+g_{i j}(\vec{x}) d x^{i} d x^{j}, \quad \vec{x}=x^{j}, \quad i, j=1, \ldots, N,
$$

The one-loop partition function is given by

$$
Z[g]=\exp \left(-S_{c}\left[\phi_{c}, g\right]\right) \int d[\tilde{\phi}] \exp \left(-\frac{1}{2} \int \tilde{\phi} A \tilde{\phi} d^{D} x\right),
$$

where the operator $A$ has the form

$$
A=-g^{00}\left(\partial_{\tau}-\mu\right)^{2}-\Delta_{N}+m^{2}+\xi R,
$$

$\Delta_{N}$ being the Laplace-Beltrami operator on the $N$ dimensional hypersurface $x^{0}=\tau=$ const, $m$ and $\xi$ arbitrary parameters and $R$ the scalar curvature of the manifold. For the sake of completeness, we consider the combination $\partial_{\tau}-\mu$, which is relevant in the finite temperature theories with chemical potential.

The ultrastatic metric $g_{\mu \nu}^{\prime}$ can be related to the static one by the conformal transformation

$$
g_{\mu \nu}^{\prime}(\vec{x})=e^{2 \sigma(\vec{x})} g_{\mu \nu}(\vec{x}),
$$

$\sigma(\vec{x})$ being a scalar function. We have to choose $\sigma(\vec{x})=-\frac{1}{2} \ln g_{00}$. In this manner, $g_{00}^{\prime}=1$ and

$g_{i j}^{\prime}=g_{i j} / g_{00}$ (optic metric). 
Recalling that by a conformal transformation

$$
\begin{aligned}
R^{\prime} & =e^{-2 q \sigma}\left[R-2(D-1) \Delta_{D} \sigma-(D-1)(D-2) g^{\mu \nu} \partial_{\mu} \sigma \partial_{\nu} \sigma\right], \\
\tilde{\phi}^{\prime} & =e^{q \sigma} \tilde{\phi} \\
\Delta_{D}^{\prime} \tilde{\phi}^{\prime} & =e^{-q \sigma}\left[\Delta_{D}-\frac{D-2}{2} \Delta_{D} \sigma-\frac{(D-2)^{2}}{4} g^{\mu \nu} \partial_{\mu} \sigma \partial_{\nu} \sigma\right] \tilde{\phi} \\
& =e^{-\sigma}\left[\Delta_{D}+\xi_{D}\left(e^{2 \sigma} R^{\prime}-R\right)\right] \tilde{\phi},
\end{aligned}
$$

where $\xi_{D}=(D-2) / 4(D-1)$ is the conformal factor, $R^{\prime}$ and $\Delta_{D}^{\prime}$ the scalar curvature and Laplace operator in the metric $g^{\prime}$, one obtains

$$
A \tilde{\phi}=e^{\sigma}\left\{-g^{\prime 00}\left(\partial_{\tau}-\mu\right)^{2}-\Delta_{N}^{\prime}+\xi_{D} R^{\prime}+e^{-2 \sigma}\left[m^{2}+\left(\xi-\xi_{D}\right) R\right]\right\} \tilde{\phi}^{\prime} .
$$

From the latter equation we have $\tilde{\phi} A \tilde{\phi}=\tilde{\phi}^{\prime} A^{\prime} \tilde{\phi}^{\prime}$, where, by definition

$$
A^{\prime}=e^{-\sigma} A e^{-\sigma}=-g^{\prime 00}\left(\partial_{\tau}-\mu\right)^{2}-\Delta_{N}^{\prime}+\xi_{D} R^{\prime}+e^{-2 \sigma}\left[m^{2}+\left(\xi-\xi_{D}\right) R\right] .
$$

This means that the action $S^{\prime}=S$ by definition. Note that classical conformal invariance requires the action to be invariant in form, that is $S^{\prime}=S[\phi, g]$, as to say $A^{\prime}=A$. As is well known, this happens only for conformally coupled massless fields $\left(\xi=\xi_{D}\right)$.

For the one-loop partition function we have

$$
Z^{\prime}=J\left[g, g^{\prime}\right] Z,
$$

where $J\left[g, g^{\prime}\right]$ is the Jacobian of the conformal transformation. Such a Jacobian can be computed for any infinitesimal conformal transformation (see for example [57] and references cited therin). To this aim it is convenient to consider a family of continuous conformal transformations

$$
g_{\mu \nu}^{q}=e^{2 q \sigma} g_{\mu \nu}
$$

in such a way that the metric is $g_{\mu \nu}$ or $g_{\mu \nu}^{\prime}$ according to whether $q=0$ or $q=1$ respectively. In this manner one has

$$
\ln J\left[g_{q}, g_{q+\delta q}\right]=\ln \frac{Z_{q+\delta q}}{Z_{q}}=\frac{\delta q}{(4 \pi)^{D / 2}} \int k_{D}\left(x \mid A_{q}\right) \sigma(x) \sqrt{g^{q}} d^{D} x,
$$

where $k_{D}\left(x \mid A_{q}\right)$, is the Seeley-DeWitt coefficient, which in the case of conformal invariant theories, is proportional to the trace anomaly.

The Jacobian for a finite transformation can be obtained from Eq. (2.55) by an elementary integration in $q$ [57]. In particular we have

$$
\ln J\left[g, g^{\prime}\right]=\frac{1}{(4 \pi)^{D / 2}} \int_{0}^{1} d q \int k_{D}\left(x \mid A_{q}\right) \sigma(x) \sqrt{g^{q}} d^{D} x,
$$

and finally

$$
\ln Z=\ln Z^{\prime}-\ln J\left[g, g^{\prime}\right] .
$$

Then we see that in principle the knowledge of the partition function $Z^{\prime}$ in the ultrastatic manifold and the heat coefficient $k_{D}\left(A_{q}\right)$ are sufficient in order to get the partition function in the static manifold. We know that the heat coefficients depend on invariant quantities build up with curvature (field strength) and their derivatives. As a consequence, they do not depend on the parameter $\mu$, since we may regard such a parameter as the temporal component of a (pure gauge) abelian potential. Thus we can simply put $\mu=0$ in the computation of the Jacobian determinant $J\left[g, g^{\prime}\right]$ using Eq. (2.56). 


\subsection{Finite temperature effects}

A reason to consider finite temperature quantum field theories is mainly based on the recent developments of cosmological models. According to the standard Big-Bang cosmology and the more recent inflationary models, the very early universe has passed through a phase of thermal equilibrium at very high temperature and density, where the symmetry was restored but with a large cosmological constant. As the universe has become cool, it has gone through several phase transitions (see for example Ref. [61]). Although the usual thermodynamical concepts may be inappropriate in the presence of very strong gravitational interactions, the need for considering finite temperature field theory in a curved background has been arisen. Strictly speaking, it has been shown that thermal equilibrium can be maintained for conformally invariant field theories in conformally flat expanding space-times [62]. Otherwise the expansion must be nearly adiabatic [63, 64, 65, 66, 67]. On the other hand, particle production in a hot Friedman universe is exponentially suppressed due to the increase of the mass by thermal effects at temperatures smaller than the Planck one [68] and the expansion should be nearly adiabatic. Phase transitions in de Sitter space-time have been first considered in Ref. [69] with the important result that critical behaviour strongly depends on curvature. Very little is known about them in anti-de Sitter space-time, mainly because de Sitter is more relevant than anti-de Sitter in inflationary scenarios.

Now we would like to present a survey of finite temperature quantum field theory and discuss some useful representations of thermodynamical quantities.

\subsubsection{The free energy}

To begin with, let us consider a (scalar) field in thermal equilibrium at finite temperature $T=1 / \beta$. It is well known that the corresponding partition function $Z_{\beta}$ may be obtained, within the path integral approach, simply by Wick rotation $\tau=i x^{0}$ and imposing a $\beta$ periodicity in $\tau$ for the field $\phi\left(\tau, x^{i}\right)(i=1, \ldots, N, N=D-1)$ [16, 70, 71, 72]. In this way the one loop approximation reads

$$
Z_{\beta}\left[\phi_{c}, g\right]=e^{-S_{c}\left[\phi_{c}, g\right]} \int_{\tilde{\phi}\left(\tau, x^{i}\right)=\tilde{\phi}\left(\tau+\beta, x^{i}\right)} d[\tilde{\phi}] \exp \left(-\int_{0}^{\beta} d \tau \int \tilde{\phi} A \tilde{\phi} d^{N} x\right) .
$$

If the space-time is ultrastatic then we are dealing with the manifold $S^{1} \times \mathcal{M}^{\mathcal{N}}$. The relevant differential operator is $A=L_{D}=-\partial_{\tau}^{2}+L_{N}$ and its eigenvalues are given by Eq. (2.26). Then, using Eqs. (2.6) and (2.31)-(2.33) we obtain [73, 74]

$$
\begin{aligned}
\ln Z_{\beta}^{(1)}+\nu \beta \zeta^{(r)}\left(-1 / 2 \mid L_{N} \ell^{2}\right) & =\frac{\nu \beta}{\pi} \sum_{n=1}^{\infty} \lim _{s \rightarrow 0} \int_{-\infty}^{\infty} e^{i n \beta t} \zeta\left(s \mid L_{N}+t^{2}\right) d t \\
& =\frac{\nu \beta}{\sqrt{\pi}} \sum_{n=1}^{\infty} \int_{0}^{\infty} t^{-3 / 2} e^{-n^{2} \beta^{2} / 4 t} K\left(t \mid L_{N}\right) d t \\
& =\frac{\nu \beta}{\pi i} \int_{\operatorname{Re} z=c} \zeta_{R}(z) \Gamma(z-1) \zeta\left(\frac{z-1}{2} \mid L_{N}\right) \beta^{-z} d z,
\end{aligned}
$$

where $\nu=1(\nu=1 / 2)$ for charged (neutral) scalar fields. Eqs. (2.59)-(2.61) not only define the finite temperature properties of quantum fields but, as we shall see in Sec. 2.7, they will be our starting point for the computation of the regularized vacuum energy.

The free energy is related to the canonical partition function by means of equation

$$
F(\beta)=-\frac{1}{\beta} \ln Z_{\beta}=F_{0}+F_{\beta},
$$

where $F_{\beta}$ represents the temperature dependent part (statistical sum) and so Eqs. 2.59)-(2.61) give different representations of $F_{\beta}$. 


\subsubsection{The thermodynamic potential}

The generalization to the more general case of a charged (scalar) field with a non vanishing chemical potential $\mu$, in thermal equilibrium by some unspecified process at finite temperature $T=1 / \beta$ is quite immediate. The grand canonical partition function has the path integral representation [75, 76, 77, 78]

$$
\begin{aligned}
Z_{\beta, \mu} & =e^{-S_{c}} \int_{\phi\left(\tau, x^{i}\right)=\phi\left(\tau+\beta, x^{i}\right)} d[\bar{\phi}] d[\phi] \exp \left(-\int_{0}^{\beta} d \tau \int \bar{\phi} A(\mu) \phi d^{N} x\right) \\
& =\exp [-\beta \Omega(\beta, \mu)],
\end{aligned}
$$

where now, the operator $A$ depends on the chemical potential $\mu$, i.e. $A(\mu)=-\left(\partial_{\tau}-\mu\right)^{2}+L_{N}$ and the latter equation defines the thermodynamic potential $\Omega(\beta, \mu)$. The operator $A(\mu)$ is still elliptic but not hermitian, in fact it is normal and its eigenvalues are complex and read

$$
\lambda_{n, j}=\left(\frac{2 \pi n}{\beta}+i \mu\right)^{2}+\omega_{j}^{2} \quad n=0, \pm 1, \pm 2, \ldots
$$

$\omega_{j}^{2}$ being the eigenvalues of $L_{N}$. Nevertheless, one can still define the related $\zeta$-function. One formally has

$$
\begin{aligned}
\Omega(\beta, \mu) & =\frac{1}{\beta} S_{c}\left[\phi_{c}, g\right]+\frac{1}{\beta} \ln \operatorname{det}\left[A(\mu) \ell^{2}\right] \\
\ln \operatorname{det} A & =\sum_{n, j} \ln \left[\omega_{j}^{2}+(2 \pi n / \beta+i \mu)^{2}\right]=\sum_{n, j} \int \frac{d \omega_{j}^{2}}{\omega_{j}^{2}+(2 \pi n / \beta+i \mu)^{2}} \\
& =\frac{\beta}{2} \sum_{j} \int\left[\operatorname{coth} \frac{\beta\left(\omega_{j}+\mu\right)}{2}+\operatorname{coth} \frac{\beta\left(\omega_{j}-\mu\right)}{2}\right] \frac{d \omega_{j}}{\omega_{j}}
\end{aligned}
$$

and integrating on $\omega_{j}$ and summing over all $j$ the well known result

$$
\begin{aligned}
\Omega(\beta, \mu) & =\sum_{j} \omega_{j}+\frac{1}{\beta} \sum_{j} \ln \left(1-e^{-\beta\left(\omega_{j}+\mu\right)}\right)+\frac{1}{\beta} \sum_{j} \ln \left(1-e^{-\beta\left(\omega_{j}-\mu\right)}\right) \\
& =\sum_{j} \omega_{j}+\frac{1}{\beta} \operatorname{Tr} \ln \left(1-e^{-\beta Q^{+}}\right)+\frac{1}{\beta} \operatorname{Tr} \ln \left(1-e^{-\beta Q^{-}}\right)
\end{aligned}
$$

follows. Above, we have introduced the two pseudo-differential operators $Q^{ \pm}=L_{N}^{1 / 2} \pm \mu$, whose eigenvalues are $\omega_{j} \pm \mu$. We see that $\Omega(\beta, \mu)$ is the sum of vacuum energy (zero-temperature contribution, formally divergent) and two finite-temperature contributions, one for particles and one for anti-particles. We also see from Eq. (2.63), that the temperature contribution, as a function of the complex parameter $\mu$, has branch points when $\mu^{2}$ is equal to an eigenvalue $\omega_{j}^{2}$. Then $\Omega_{\beta}(\beta, \mu)$ is analytic in the $\mu$ complex plain with a cut from $\omega_{0}$ to $\infty$ and from $-\omega_{0}$ to $-\infty$. Thus, the physical values of $\mu$ are given by $|\mu| \leq \omega_{0}$.

If one makes use of the $\zeta$-function regularization, one obtains

$$
\Omega(\beta, \mu)=\frac{1}{\beta} S_{c}\left[\phi_{c}, g\right]-\frac{1}{\beta} \zeta^{\prime}\left(0 \mid A(\mu) \ell^{2}\right) .
$$

Using Eq. (D.1) and Mellin representation of $\zeta$-function, Eq. 2.13), after some manipulations similar to the ones of Sec. 2.2.2, one gets the three useful representations for the temperature 
dependent part $\Omega_{\beta}(\beta, \mu)$ of the thermodynamic potential (2.64) [74, 79]

$$
\begin{aligned}
\Omega_{\beta}(\beta, \mu) & =\Omega(\beta, \mu)-\Omega_{0} \\
& =-\frac{1}{\pi} \sum_{n=1}^{\infty} \int_{-\infty}^{\infty} e^{i n \beta t} \zeta^{\prime}\left(0 \mid L_{N}+[t+i \mu]^{2}\right) d t \\
& =-\frac{1}{\sqrt{\pi}} \sum_{n=1}^{\infty} \cosh n \beta \mu \int_{0}^{\infty} t^{-3 / 2} e^{-n^{2} \beta^{2} / 4 t} K\left(t \mid L_{N}\right) d t \\
& =-\frac{1}{\pi i} \sum_{n=0}^{\infty} \frac{\mu^{2 n}}{(2 n) !} \int_{\operatorname{Re} s=c} \zeta_{R}(s) \Gamma(s+2 n-1) \zeta\left(\frac{s+2 n-1}{2} \mid L_{N}\right) \beta^{-s} d s .
\end{aligned}
$$

Here $\Omega_{0}=\frac{1}{\beta} S_{c}\left[\phi_{c}, g\right]+\zeta^{(r)}\left(-1 / 2 \mid L_{N} \ell^{2}\right)$ is the zero-temperature contribution (classical and vacuum energy). Eqs. (2.65)-(2.67) are valid for any $|\mu|<\omega_{0}, \omega_{0}$ being the smallest eigenvalue of $L_{N}$. When $|\mu|=\omega_{0}$ a more careful treatment is needed. The physical reason for this behaviour is the occurrence of Bose-Einstein condensation. Of course, in the limit $\mu \rightarrow 0$, Eqs. (2.65)-(2.67) reduces to Eqs. (2.59)-(2.61) which has been discussed in the previous section.

For the sake of completeness, we report another integral representation of the thermodynamic potential, which can be obtained in a similarly to the one used for deriving Eq. 22.67) [79]. To this aim, we observe that the poles of $\zeta\left(s \mid Q^{ \pm}\right)$(related to the two pseudo-differential operators $\left.Q^{ \pm}\right)$are given by Seeley theorem [29] at the points $s=N-k(k=0,1, \ldots)$ with residues which are polynomials in $\mu$. The formula which generalizes the result of Ref. 80 to compact manifolds then reads

$$
\Omega_{\beta}(\beta, \mu)=-\frac{1}{2 \pi i} \int_{\operatorname{Re} s=c} \zeta_{R}(s) \Gamma(s-1)\left[\zeta\left(s-1 \mid Q^{+}\right)+\zeta\left(s-1 \mid Q^{-}\right)\right] \beta^{-s} d s .
$$

This should be used to discuss $\Omega_{\beta}(\beta, \mu)$ as a function of complex $\mu$.

\subsection{The regularization of vacuum energy}

The vacuum energy density (the first term on the right hand side in Eq. (2.63)) is the only source of divergences in the thermodynamic potential. Therefore we shall briefly discuss how to give it a mathematical meaning. We present a general formula for the vacuum energy of a scalar field defined on an ultrastatic space-time with compact spatial section consisting in general in a manifold with boundary.

We start with some general considerations. In the last decade there has been un increasing interest in investigating vacuum effects or zero point fluctuations in the presence of boundaries [81] or in space-time with non trivial topology. The introduction of boundaries and related boundary conditions on quantum fields may be considered as an excellent idealization of complicated matter configurations. This is certainly true for the original Casimir configuration, namely two neutral parallel conducting plates placed at a distance L. In fact the interpretation due to Casimir of the attractive force present in such a configuration (experimentally verified) seems to support such a point of view. In this case, the boundary conditions associated with the electromagnetic field are the perfect conductor boundary conditions and the related Casimir energy is negative. Casimir conjectured the same thing to be hold for a spherical shell. In this way the fine structure constant could have been determined.

The computation for the spherical shell was performed in Refs. [82, 83, 84] and the result was a positive vacuum energy, proving that the Casimir conjecture was wrong (see however Ref. [85], where interpreting the Casimir effect as a screening effect, a negative energy is obtained for a scalar field and also Ref. [86] for a recent treatment). Other computations associated with different geometries of the boundary seems to support the idea that the sign of Casimir energy depends on the cavity in a non trivial way. 
On general ground, as it has been stressed by DeWitt [1, 87], the introduction of boundaries or the identification of surfaces modifies the global topology of a local space-time. As a consequence one is effectively dealing with quantum field theory on a curved manifold.

We would like to mention that Casimir energy plays an important role in hadronic physics where, due to the confinement of quark and gluon fields, vacuum effects cannot be neglected as soon as one is working in the framework of bag models [88]. In such situation, one is forced to consider a compact cavity. For computational reasons, only the case of a spherical cavity has been investigated in some detail (see for example Ref. [89]).

For the sake of simplicity, here we shall mainly deal with a scalar field defined on a $D$ dimensional space-time $\mathcal{M}^{D}$ with a $N=(D-1)$-dimensional smooth boundary. The metric is supposed to be ultrastatic, then the results of Sec. 2.6 can be applied. Vector and spinor fields may be treated along the same line, paying attention however to the related boundary conditions.

Given an arbitrarily shaped cavity, one may consider an internal and an external problem. For the sake of simplicity, we shall assume that the internal problem is associated with a simply connected manifold. The external problem is usually associated with a double connected manifold. The reason stems from the necessity to introduce a very "large" manifold in order to always deal with a compact manifold. This large manifold may be considered as a reference manifold, namely a volume cut-off of Minkowski space-time. Its boundary can be pushed to infinity at the end of the computation 83]. As far as the boundary conditions associated with the external problem, we shall mainly leave understood the use of the same internal boundary conditions, paying attention however to the proposal of Ref. [90], in which the external boundary condition is different from the internal one.

To start with, we shall consider a compact arbitrarily shaped manifold with boundary, which may be multi-connected. The main issue related to vacuum energy $E_{v}$ is the necessity of a regularization scheme. In fact, if one naively makes use of canonical quantization of a scalar field, the result is (see for example Ref. 91])

$$
E_{v}=\nu \sum_{j} \omega_{j}
$$

$\omega_{n}^{2}$ being the eingenvalues of $L_{N}$ defined on $\mathcal{M}^{N}$, the eigenfunctions of which are the mode functions satisfying suitable conditions on the boundary of $\mathcal{M}^{N}$ and, as in Sec. 2.6, $\nu=1(\nu=$ $1 / 2$ ) for charged (neutral) scalar fields. As we have seen in Sec. 2.6, also formal manipulations lead to such an expression like Eq. (2.69). Of course it is an ill defined object and one needs some prescriptions in order to extract a finite observable quantity.

In order to regularize Eq. (2.69) by the use of $\zeta$-function, one may try to write

$$
E_{v}=\nu \lim _{s \rightarrow-\frac{1}{2}} \zeta\left(s \mid L_{N}\right) \text {. }
$$

Indeed, in the case of hyper-rectangular cavities and massless free fields, this expression gives a result in agreement with other regularizations [92]. However, Eq. (2.70) does not work when one is dealing with an arbitrarily shaped cavity. This can be easily understood looking at Eq. (2.20). From such an equation we see in fact that for $s \rightarrow-1 / 2, \zeta\left(s \mid L_{N}\right)$ has in general a simple pole, the residue of which is given by $-K_{D}\left(L_{N}\right) / \sqrt{4 \pi}$. As a consequence the regularization given by Eq. (2.70) is meaningless, unless $K_{D}\left(L_{N}\right)$ vanishes. This is just the case of massless free fields in hyper-rectangular cavities.

A possible definition of vacuum energy in terms of $\zeta$-function, which is valid for a manifold with an arbitrary smooth boundary, can be obtained starting from the finite temperature partition function $Z_{\beta}$. According to Ref. 933, we define

$$
E_{v}=-\lim _{\beta \rightarrow \infty} \partial_{\beta} \ln Z_{\beta}
$$


Using for $\ln Z_{\beta}$ the regularized expression (2.60), one immediately gets (here $S_{c}\left[\phi_{c}, g\right]=0$ because we are considering free fields)

$$
E_{v}=\nu \zeta^{(r)}\left(-1 / 2 \mid L_{N} \ell^{2}\right) .
$$

With regard to this result, we would like to observe that a similar prescription has been appeared in many places in the literature (see for example [94, 53]). Here we would like to stress the proposal contained in Ref. [95], which is very close to our approach. In other words, the pathintegral $\zeta$-function derivation presented here, leads directly to the "principal part prescription" of Ref. [95]. It is interesting to observe that the term in the vacuum energy, which depends on $\ell$ (see Eq. (2.34)), is proportional to the integral of the conformal anomaly [2].

The total contribution (interior plus exterior minus the reference one) to vacuum energy is relevant from the physical point of view, for example in the classic electromagnetic Casimir effect associated to a conducting cavity or in some extensions of the bag model. If one is dealing with a free massless field on a $3+1$ dimensional manifold, as in the electromagnetic case, it turns out that the total Casimir energy seems to be free from the ambiguity associated with $\ell$ [83. As we shall see in Sec. 4.4.2, the ambiguity due to the free parameter $\ell$ can be removed by renormalization when the theory has a natural scale. This is true for massive or self-interacting fields, but also for massless fields on curved manifolds. 


\section{Constant curvature manifolds}

The present section is devoted to the derivation of heat kernel and $\zeta$-function related to the Laplace-Beltrami operator acting on fields living on compact manifolds with constant curvature. In the spirit of the report, we describe general aspects, relegating specific results to the appendices.

For a detailed discussion of $N$ dimensional torus and sphere, we refer the reader to the vast literature on the subject (for a recent review see for example Ref. [6] and references therein). Here we shall very briefly describe some useful techniques and we shall give some known, but also less known representations for $\zeta$-function, which shall be used in the physical applications. On the contrary, compact hyperbolic manifolds shall be analyzed in some detail, because we suppose the reader to be not familiar with the hyperbolic geometry, the related isometry groups and the Selberg trace formula (see, for example, Refs. [96, 97, 13] and references therein).

\subsection{The heat kernel and $\zeta$-function on the torus}

Here we perform the analytic continuation for the $\zeta$-function of the operator $\mathrm{E}_{N}=-\Delta_{T^{N}}+\alpha^{2}$ acting on twisted scalar fields on the torus $T^{N}$. $T^{N}$ is the direct product of $N$ circles $S^{1}$ with radii $r_{i}$. In order to take into account of twists, we introduce a $N$-vector $\vec{q}$ with components 0 or $1 / 2$, in such a manner that the eigenvalues of the Laplacian have the form $(\vec{k}+\vec{q}) \cdot \mathcal{R}_{N}^{-1}(\vec{k}+\vec{q})$, $\mathcal{R}_{N}$ being the diagonal matrix $\operatorname{diag}\left(\mathcal{R}_{N}\right)=\left(r_{1}^{2}, \ldots, r_{N}^{2}\right)$.

The trace of the heat kernel $K\left(t \mid \mathrm{E}_{N}\right)=\operatorname{Tr} \exp \left(-t \mathrm{E}_{N}\right)$ on the torus can be directly derived by the factorization property (2.11) knowing the kernel on $S^{1}$. So we have

$$
K\left(t \mid \mathrm{E}_{N}\right)=e^{-t \alpha^{2}} \sum_{\vec{k}} e^{-t(\vec{k}+\vec{q}) \cdot \mathcal{R}_{N}^{-1}(\vec{k}+\vec{q})}=\frac{\Omega_{N} e^{-t \alpha^{2}}}{(4 \pi t)^{\frac{N}{2}}} \sum_{\vec{k}} e^{-2 \pi i \vec{k} \cdot \vec{q}} e^{-\pi^{2}\left(\vec{k} \cdot \mathcal{R}_{N} \vec{k}\right) / t},
$$

where $\vec{k} \in \mathbb{Z}^{N}$ and $\Omega_{N}=(2 \pi)^{N} \sqrt{\operatorname{det} \mathcal{R}_{N}}$ is the hypersurface of the torus. The latter equation has been derived by using Eq. (B.15) in Appendix B.2.

As it is well known, the $\zeta$-function on the torus is given in terms of Epstein $Z$-function (see Appendix $\mathrm{D}$ for definition and properties). In fact, one directly obtains

$$
\zeta\left(s \mid \mathrm{E}_{N}\right)=Z_{\mathcal{R}_{N}^{-1}}\left(\frac{2 s}{N} ; \vec{q}, 0\right) .
$$

Other useful representations can be obtained by making use of Mellin representation (2.13). They read

$$
\begin{aligned}
\zeta\left(s \mid \mathrm{E}_{N}\right) & -\frac{\Omega_{N} \Gamma(s-N / 2) \alpha^{N-2 s}}{(4 \pi)^{\frac{N}{2}} \Gamma(s)}=\frac{2 \Omega_{N} \alpha^{N-2 s}}{(4 \pi)^{\frac{N}{2}} \Gamma(s)} \sum_{\vec{k} \neq 0} \frac{e^{-2 \pi i \vec{k} \cdot \vec{q}} K_{N / 2-s}\left(2 \pi \alpha\left[\vec{k} \cdot \mathcal{R}_{N} \vec{k}\right]^{\frac{1}{2}}\right)}{\left(\pi \alpha\left[\vec{k} \cdot \mathcal{R}_{N} \vec{k}\right]^{\frac{1}{2}}\right)^{\frac{N}{2}-s}} \\
= & \frac{\Omega_{N} \alpha^{N-2 s}}{(4 \pi)^{\frac{N-1}{2}} \Gamma(s) \Gamma\left(\frac{N+1}{2}-s\right)} \sum_{\vec{k} \neq 0} e^{-2 \pi i \vec{k} \cdot \vec{q}} \int_{1}^{\infty}\left(u^{2}-1\right)^{\frac{N-1}{2}-s} e^{-2 \pi \alpha u\left[\vec{k} \cdot \mathcal{R}_{N} \vec{k}\right]^{\frac{1}{2}}} d u .
\end{aligned}
$$

The representation of $\zeta$ given by Eq. (3.3) is valid for any $s$, since the Mc Donald functions $K_{\nu}(z)$ are exponentially vanishing for $z \rightarrow \infty$ and so the series is convergent (here $\alpha>0$ ). On the contrary, the representation (3.4) is only valid for $\operatorname{Re} s<N / 2$, but this is what we need in the evaluation of physical quantities. It has to be remarked that the right hand sides of the above formula, in the limit $\alpha \rightarrow 0$, give exactly the $\zeta$-function in the massless case. 
The $\zeta$-function on $T^{Q} \times \mathcal{M}^{\mathcal{N}}$ Here we just write down the Mellin-Barnes representation of $\zeta$-function, Eq. 2.25), for this particular case, $\mathcal{M}^{\mathcal{N}}$ being an arbitrary compact manifold. The operator is assumed to be of the form $A=-\Delta_{Q}+L_{N}$, with $\Delta_{Q}$ the Laplace operator in $T^{Q}$ and $L_{N}$ a differential operator in $M^{N}$. For the $\zeta$-function related to $\Delta_{Q}$ we use the representation in terms of Epstein $Z$-function (see Eq. (3.2)). Then, supposing $L_{N}$ to be an invertible operator, from Eq. (2.25) we obtain

$$
\zeta(s \mid A)=\frac{1}{2 \pi i \Gamma(s)} \int_{\operatorname{Re} z=c} \Gamma\left(\frac{s}{2}+z\right) \zeta\left(\frac{s}{2}+z \mid L_{N}\right) \Gamma\left(\frac{s}{2}-z\right) Z_{\mathcal{R}_{Q}^{-1}}\left(\frac{s-2 z}{Q} \mid \vec{q}, 0\right) d z .
$$

Here we have implicitly assumed also $\Delta_{Q}$ to be an invertible operator. This happens when there is at least one twist. If $\vec{q}=0$ (no twists), there is a zero-mode and to take account of it on the right hand side of the latter equation we must add $\zeta\left(s \mid L_{N}\right)$. In Eq. (3.5), $c$ must satisfy the condition $-\frac{s-Q}{2}<c<\frac{s-N}{2}$, which is quite restrictive for physical aims. Such a restriction can be relaxed if we compute $\zeta$-function by a technique similar to the one used in Sec. 2.2.2. In this way we have

$$
\begin{aligned}
\zeta(s \mid A)= & \frac{\Omega_{Q}}{(4 \pi)^{Q / 2} \Gamma(s)}\left[\Gamma\left(s-\frac{Q}{2}\right) \zeta\left(s-\frac{Q}{2} \mid L_{N}\right)\right. \\
& \left.+\frac{1}{2 \pi i} \int_{\operatorname{Re} z=c} \pi^{-\frac{z}{2}} \Gamma\left(s+\frac{z-Q}{2}\right) \zeta\left(s+\frac{z-Q}{2} \mid L_{N}\right) \Gamma\left(\frac{z}{2}\right) Z_{\mathcal{R}_{Q}}\left(\frac{z}{Q} \mid 0,-\vec{q}\right) d z\right],
\end{aligned}
$$

which reduces to Eq. (2.30) for $Q=1$ and $q=0$. Now $c>N+Q$. Similar expressions can be also obtained for manifolds with other kinds of sections, for example spheres or hyperbolic manifolds.

\subsection{Representations and recurrence relations for $\zeta$-function on the sphere}

Here we shall review some techniques used in the literature, which provide useful analytical extensions for $\zeta$-function of Laplacian on spheres (compact rank-one symmetric spaces). We shall explain the known method based on binomial expansion, which has been recently used in Ref. 98] in order to evaluate $\zeta$-function on orbifold-factored spheres $S^{N} / \Gamma, \Gamma$ being a group of isometry. We shall closely follow Ref. [6]. We would like to mention the pionering work by Minakshisundaram, who first introduced these kind of generalized zeta functions [99, 100]. His results are very close to the ones we shall obtain for the hyperbolic case. Recently, these results have been derived by a different technique also in Ref. 101. Finally we shall also give an integral representation in the complex plane for the trace of an arbitrary function of the Laplacian acting on scalar fields in $S^{N}$ and we shall derive some recurrence relations for the trace of the heat kernel and for the $\zeta$-function density. Here we normalize the constant curvature to $\kappa=1$.

To start with, we recall that for compact rank-one symmetric spaces the spectrum and its degeneration are at disposal (see for example Refs. [102, 6]). In particular, for the eigenvalues $\lambda_{n}$ and their degeneration $d_{n}^{N}$ of the Laplace operator $-\Delta_{N}$ on $S^{N}$ we have

$$
\lambda_{n}=n\left(n+2 \varrho_{N}\right), \quad \quad d_{n}^{N}=\frac{2\left(n+\varrho_{N}\right) \Gamma\left(n+2 \varrho_{N}\right)}{\Gamma\left(2 \varrho_{N}+1\right) \Gamma(n+1)}, \quad n \geq 0,
$$

with $\varrho_{N}=(N-1) / 2$. Eqs. (3.6) are valid for any $N$, but $d_{0}^{1}=1$. One can see that the degeneration of eigenvalues for odd $N$ (respectively even $N$ ) is an even (respectively odd) polynomial of $N-1$ degree in $n+\varrho_{N}$. In fact we have

$$
d_{0}^{1}=1, \quad d_{n}^{1}=2, \quad d_{n}^{2}=2\left(n+\varrho_{2}\right),
$$




$$
\begin{array}{ll}
d_{n}^{N}=\frac{2}{(N-1) !} \prod_{k=0}^{\frac{N-3}{2}}\left[\left(n+\varrho_{N}\right)^{2}-k^{2}\right], & \text { for odd } N \geq 3, \\
d_{n}^{N}=\frac{2\left(n+\varrho_{N}\right)}{(N-1) !} \prod_{k=0}^{\frac{N-4}{2}}\left[\left(n+\varrho_{N}\right)^{2}-\left(k+\frac{1}{2}\right)^{2}\right], & \text { for even } N \geq 4 .
\end{array}
$$

Then we can define the coefficients $a_{k}^{N}$ by means of equation

$$
d_{n}^{N}=\Omega_{N} c_{n}^{N}=\Omega_{N} \sum_{k=1}^{N-1} a_{k}^{N}\left(n+\varrho_{N}\right)^{k},
$$

valid for any $N \geq 2$. Here $\Omega_{N-1}=2 \pi^{\frac{N}{2}} / \Gamma\left(\frac{N}{2}\right)$ is the volume (hypersurface) of $S^{N-1}$, which we shall explicitly write in all formulae in order to point out the strictly similarity with the compact hyperbolic case, which shall be extensively treated in Sec. 3.4.5. The case $N=1$ is quite trivial and will be treated separately. It can be also considered as a particular case of previous section. To consider both even and odd cases, we have written a general polynomial, but of course only even or odd coefficients $a_{k}^{N}$ are non vanishing, according to whether $N$ is odd or even.

By definition, heat kernel and $\zeta$-function are given by

$$
\begin{aligned}
& K\left(t \mid \mathrm{E}_{N}\right)=\Omega_{N} e^{-t \alpha^{2}} \sum_{n=0}^{\infty} c_{n}^{N} e^{-t\left(n+\varrho_{N}\right)^{2}}, \\
& \zeta\left(s \mid \mathrm{七}_{N}\right)=\Omega_{N} \sum_{n=0}^{\infty} c_{n}^{N}\left[\left(n+\varrho_{N}\right)^{2}+\alpha^{2}\right]^{-s} .
\end{aligned}
$$

For more generality, we have considered the massive operator $\mathrm{t}_{N}=-\Delta_{S^{N}}+\alpha^{2}+\kappa \varrho_{N}^{2}$, $\alpha$ being an arbitrary constant. The choices $\alpha^{2}=m^{2}-\varrho_{N}^{2}$ corresponds to minimal coupling while the choice $\alpha^{2}=0$ correspond to conformal coupling (we think of $S^{N}$ as the spatial section of an ultrastatic $N+1$ dimensional manifold). As usual zero modes must be omitted in Eq. (3.10). Because of homogeneity of $S^{N}, K_{t}^{S^{N}}(x, x)$ does not depend on $x$ and so, a part the volume, Eq. (3.9) determines also the heat kernel in the coincidence limit.

\subsubsection{The series representation}

Using Eq. (3.8) in Eq. (3.10) and making a binomial expansion we obtain a representation of $\zeta\left(s \mid \mathrm{E}_{N}\right)$ as an infinite sum of Riemann-Hurwitz $\zeta_{H}$-functions (See Appendix D, Eq. (D.4)). For any $N \geq 2$ and $\left|\alpha^{2}\right|<\varrho_{N}^{2}$ (remember that the curvature is normalized to $\kappa=1$ ) it reads

$$
\zeta\left(s \mid \mathrm{E}_{N}\right)=\Omega_{N} \sum_{n=0}^{\infty} \sum_{k=1}^{N-1} a_{k}^{N} \frac{(-1)^{n} \Gamma(s+n) \alpha^{2 n}}{\Gamma(n+1) \Gamma(s)} \zeta_{H}\left(2 s+2 n-k ; \varrho_{N}\right) .
$$

By the same method, for $N=1$ we obtain

$$
\zeta\left(s \mid \mathrm{E}_{1}\right)=\frac{1}{\alpha^{2 s}}+2 \sum_{n=1}^{\infty} \frac{(-1)^{n} \Gamma(s+n) \alpha^{2 n}}{\Gamma(n+1) \Gamma(s)} \zeta_{R}(2 s+2 n) .
$$

Eq. (3.11) notably simplify in the case of conformal coupling $(\alpha=0)$. In fact we have

$$
\zeta\left(s \mid \mathrm{E}_{N}\right)=\Omega_{N} \sum_{k=1}^{N-1} a_{k}^{N} \zeta_{H}\left(2 s-k ; \varrho_{N}\right) .
$$


For the massless minimal coupling $\left(\alpha^{2}=-\varrho_{N}^{2}\right)$, one has to pay attention to the zero mode. After the subtraction of it one obtains

$$
\zeta\left(s \mid \mathrm{E}_{N}\right)=\Omega_{N} \sum_{n=0}^{\infty} \sum_{k=1}^{N-1} a_{k}^{N} \frac{\Gamma(s+n) \varrho_{N}^{2 n}}{\Gamma(s) \Gamma(n+1)} \zeta_{H}\left(2 s+2 n-k ; \varrho_{N}+1\right) .
$$

These equations are valid for $N \geq 2$. For $N=1$ we have the simpler result $\zeta\left(s \mid \mathrm{E}_{1}\right)=2 \zeta_{R}(2 s)$.

\subsubsection{Recursive representation}

Now we are going to derive a representation of $\zeta\left(s \mid \mathrm{E}_{N}\right)$ in terms od $\zeta\left(s \mid \mathrm{E}_{1}\right)$ or $\zeta\left(s \mid \mathrm{E}_{2}\right)$ according to whether $N$ is odd or even. Similar results can be found in Ref. [6]. We first consider the odd dimensional case with $N \geq 3$. Using again Eq. (3.10) and observing that $\varrho_{N}$ is an integer we easily get

$$
\begin{aligned}
\zeta\left(s \mid \mathrm{E}_{N}\right) & +\Omega_{N} \sum_{n=1}^{\varrho_{N}-1} \sum_{k=1}^{\varrho_{N}} a_{2 k}^{N} n^{2 k}\left(n^{2}+\alpha^{2}\right)^{-s} \\
& =\left.\Omega_{N} \sum_{n=1}^{\infty} \sum_{k=1}^{\varrho_{N}} \frac{(-1)^{k} a_{2 k}^{N}}{\Gamma(s)} \frac{d^{k}}{d \lambda^{k}} \int_{0}^{\infty} t^{s-k-1} e^{-\lambda t\left(n^{2}+\alpha^{2} / \lambda\right)} d t\right|_{\lambda=1} \\
& =\Omega_{N} \sum_{k=1}^{\varrho_{N}} \frac{a_{2 k}^{N} \Gamma(s-k) \alpha^{-2 s}}{2 \Gamma(s)}\left(\alpha^{4} \frac{d}{d \alpha^{2}}\right)^{k}\left[\alpha^{2(s-k)} \zeta\left(s-k \mid \mathrm{E}_{1}\right)\right] .
\end{aligned}
$$

A similar equation can be obtained also in the even dimensional case with $N \geq 4$. By taking into account that now $\varrho_{N}$ is a half-integer one obtains

$$
\begin{aligned}
\zeta\left(s \mid \mathrm{E}_{N}\right) & \left.+\Omega_{N} \sum_{n=0}^{\left[\varrho_{N}\right]-1} \sum_{k=0}^{\left[\varrho_{N}\right]} a_{2 k+1}^{N}\left(n+\frac{1}{2}\right)^{2 k+1}\left[\left(n+\frac{1}{2}\right)^{2}+\alpha^{2}\right)\right]^{-s} \\
& =\left.\Omega_{N} \sum_{n=1}^{\infty} \sum_{k=0}^{\left[\varrho_{N}\right]} \frac{(-1)^{k} a_{2 k+1}^{N}(n+1 / 2)}{\Gamma(s)} \frac{d^{k}}{d \lambda^{k}} \int_{0}^{\infty} t^{s-k-1} e^{-\lambda t\left[(n+1 / 2)^{2}+\alpha^{2} / \lambda\right]} d t\right|_{\lambda=1} \\
& =\Omega_{N} \sum_{k=0}^{\left[\varrho_{N}\right]} \frac{a_{2 k+1}^{N} \Gamma(s-k) \alpha^{-2 s}}{2 \Gamma(s)}\left(\alpha^{4} \frac{d}{d \alpha^{2}}\right)^{k}\left[\alpha^{2(s-k)} \zeta\left(s-k \mid \mathrm{E}_{2}\right)\right]
\end{aligned}
$$

where $\left[\varrho_{N}\right]$ represents the integer part of $\varrho_{N}$. Eqs. (3.12) and (3.13) are valid in the absence of zero-modes. Zero-modes must be not consider in the definition of $\zeta$-function and this is equivalent to disregard (possible) singularities in the latter equations.

We know that the knowledge of $\zeta$-function on $S^{1}$ and $S^{2}$ is sufficient in order to get the $\zeta$-function on any sphere. The results for $S^{1}, S^{2}$ and $S^{3}$ are derived in Appendix $\mathrm{Q}$.

\subsubsection{A complex integral representation}

To finish the section, we exhibit an integral representation and some recurrence relations for $\zeta$-function, which look like to the ones we shall obtain for the hyperbolic case in Sec. 3.4.5.

First of all, we observe that

$$
c_{n}^{N+2}=\frac{(n+1)(n+N)}{2 \pi N} c_{n+1}^{N}
$$

from which we easily get the recurrence relations

$$
\frac{K\left(t \mid \mathrm{七}_{N+2}\right)}{\Omega_{N+2}}=-\frac{1}{2 \pi N \Omega_{N}}\left[\partial_{t}+\alpha^{2}+\kappa \varrho_{N}^{2}\right] K\left(t \mid \mathrm{七}_{N}\right),
$$




$$
\frac{\zeta\left(s \mid \mathrm{E}_{N+2}\right)}{\Omega_{N+2}}=-\frac{1}{2 \pi N \Omega_{N}}\left[\left(\alpha^{2}+\kappa \varrho_{N}^{2}\right) \zeta\left(s \mid \mathrm{E}_{N}\right)-\zeta\left(s-1 \mid \mathrm{E}_{N}\right)\right] .
$$

It is easy to see that Eq. (3.15) is also valid if we substitute the corresponding regularized quantities according to Eq. (2.34). As we shall see if Sec. 3.4.5 with small changes, Eqs. (3.14) and (3.15) are also valid on $H^{N}$. Knowing the $\zeta$-function on $S^{1}$ and $S^{2}$ and using recurrence formulae above, we obtain the $\zeta$-function on the sphere in any dimension.

In order to get the integral representation in the complex plane, we consider an analytic function $h\left(z^{2}\right)$ such that $\sum h\left(\lambda_{n}+\varrho_{N}^{2}\right)$ exists, the sum being extended to all eigenvalues (counted with their multiplicity) of $-\Delta_{N}$ on the sphere $S^{N}$. Then one can easily check that for any $N>1$ the following complex integral representation holds:

$$
\sum_{n} h\left(\lambda_{n}+\varrho_{N}^{2}\right)=\frac{\Omega_{N}}{2 \pi i} \int_{\Gamma} h\left(z^{2}\right) \Phi_{N}^{S}(z) d z
$$

where $\Gamma$ is an open path in the complex plane going (clockwise) from $\infty$ to $\infty$ around the positive real axix enclosing the point $z=\varrho_{N}$ and

$$
\Phi_{N}^{S}(z)=\frac{2 z \Gamma\left(\varrho_{N}+z\right) \Gamma\left(\varrho_{N}-z\right)}{(4 \pi)^{N / 2} \Gamma(N / 2)} \cos \pi\left(\varrho_{N}-z\right),
$$

which satisfy the recurrence formula

$$
\Phi_{N+2}^{S}(z)=\frac{\varrho_{N}^{2}-z^{2}}{2 \pi N} \Phi_{N}^{S}(z) .
$$

We have

$$
\Phi_{2}^{S}(z)=\frac{z \tan \pi z}{2}, \quad \Phi_{3}^{S}(z)=-\frac{z^{2} \cot \pi z}{2 \pi} .
$$

Using Eq. (3.16) we obtain for example

$$
\begin{aligned}
& K\left(t \mid \mathrm{E}_{2}\right)=\frac{\Omega_{2} e^{-t \alpha^{2}}}{8 i t} \int_{\Gamma} \frac{e^{-t z^{2}}}{\cos ^{2} \pi z} d z, \\
& \zeta\left(s \mid \mathrm{E}_{2}\right)=\frac{\Omega_{2}}{8 i(s-1)} \int_{\Gamma} \frac{\left(z^{2}+\alpha^{2}\right)^{-(s-1)}}{\cos ^{2} \pi z} d z .
\end{aligned}
$$

The latter equation is valid for any $s$ if we choose a suitable path, for example $z=a+r e^{ \pm i \pi / 4}$, $a$ being an arbitrary real number satisfying $|a|<1 / 2,|\alpha|<a<1 / 2,1 / 2<a<3 / 2$ according to whether $\alpha^{2}>0,0 \geq \alpha^{2}>-1 / 4, \alpha^{2}=-1 / 4$.

\subsection{Hyperbolic manifolds}

The geometry of $N$-dimensional torus and sphere are quite known. On the contrary, the hyperbolic one is less familiar. For this reason, in the following two subsections, we shall present an elementary and self-contained survey of some issues on hyperbolic geometry. For further details, we refer to the cited references.

By definition, hyperbolic manifolds are the Riemannian space forms with constant negative curvature. Actually, they can be defined for any metric signature in which case they are called pseudo-Riemannian hyperbolic space forms. The Riemann tensor of such spaces is locally characterized by the condition $R_{i j k l}=\kappa\left(g_{i k} g_{j l}-g_{i l} g_{j k}\right)$ with $\kappa$ a constant. We denote the signature of a non degenerate metric $g_{i j}$ by $(n, N-n)$, where $n$ is the number of negative eigenvalues of $g_{i j}(i, j=0, \ldots, N-1)$. The unique simply connected and flat pseudo-Riemannian manifold of signature $(n, N-n)$ is just $\mathbb{R}^{N}$ endowed with the standard diagonal metric with the given signature. The following theorem is quoted from 103: 
Theorem 1 Let $\kappa$ be non zero and $a>0$ defined by $\kappa=e a^{-2}, e= \pm 1$ and

$$
\Sigma_{n}^{N}=\left\{x \in \mathbb{R}^{N}: g_{i j} x^{i} x^{j}=e a^{2}\right\}, \quad N \geq 3,
$$

where $g_{i j}$ has signature $(n, N-n)$ and $x^{i}=\left(x^{0}, \ldots, x^{N-1}\right)$ are rectilinear coordinates on $\mathbb{R}^{N}$. Then every $\Sigma_{n}^{N}$, endowed with the induced metric, is a complete pseudo-Riemannian manifold of constant curvature $\kappa$ and signature $(n, N-n-1)$ if $e=1$ or $(n-1, N-n)$ if $e=-1$. The geodesics of $\Sigma_{n}^{N}$ are the intersections $\Pi \cap \Sigma_{n}^{N}$, $\Pi$ being a plane through the origin in $\mathbb{R}^{N}$. The group of all isometries of $\Sigma_{n}^{N}$ is the pseudo-orthogonal group $O(n, N-n)$ of the metric $g_{i j}$.

The class of spaces described by the theorem are the fundamental models for constant curvature manifolds. The models with $\kappa>0$ are the pseudo-spherical space forms, denoted by $S_{n}^{N}$, the models with $\kappa<0$ are called pseudo-hyperbolic space forms, denoted by $H_{n}^{N}$. It may be noted that $\Sigma_{N}^{N}=\emptyset$ if $e=1$ and likewise $\Sigma_{0}^{N}=\emptyset$ if $e=-1$. Thus we define $S_{n}^{N}=\Sigma_{n}^{N+1}$ and $H_{n}^{N}=\Sigma_{n+1}^{N+1}$, so for both of them the signature is $(n, N-n)$, the dimension is $N$ and no one is the empty set. From the definition it follows that $H_{n}^{N}$ is simply connected for $n \neq 0,1$. On the other hand, $H_{1}^{N}$ is connected with infinite cyclic fundamental group. From the theorem we see it has Lorentz signature $(1, N-1)$ and contains closed time-like geodesics. In fact, $H_{1}^{N}$ is what in general relativity is called the $N$-dimensional anti-de Sitter space-time (similarly, the space $S_{1}^{N}$ is de Sitter space-time) [51]. Finally we have $H_{0}^{N}$ which has two simply connected, isometric components. We denote the component with $x_{0}>a$ by $H^{N}$. This is the manifold we are mainly interested in. It can be characterized as the unique simply connected Riemannian manifold with constant negative curvature. Its isometry group is the orthochronus Lorentz group $O^{+}(1, N)$ of matrices $\Lambda_{a b}$ such that $\Lambda_{00}>0$, in order to preserve the condition $x_{0}>a$. An interesting feature of this space is that it represents the Euclidean section appropriate for anti-de Sitter space-time 104. Accordingly, it can be shown that Euclidean quantum field theory on $H^{N}$ is the Wick rotation of a field theory on the anti-de Sitter, although on this space-time there are other field representations which cannot be obtained in this way.

Next, we describe all the connected spaces with constant negative curvature and arbitrary signature. They are obtained from the spaces $H_{n}^{N}$ by the operation of taking the quotient with respect to the action of a group of isometries. But of course the group action has to be restricted in some way. For example, fixed points are going to produce singularities in the quotient manifold, as can be seen with the following example. Let $\Gamma$ be the cyclic group generated by a rotation around the origin in $\mathbb{R}^{2}$ with angle $2 \pi / \alpha$, i.e. the set of all rotations whose angle is an integer multiple of $2 \pi / \alpha$. The quotient manifold (i.e. the set of orbits) $\mathbb{R}^{2} / \Gamma$ is then a cone with angle $2 \pi / \alpha$ at the vertex, which is also the fixed point of $\Gamma$. Note that the cone is a metric space but the infinitesimal metric is singular at the vertex.

When a group $\Gamma$ acts on a space without fixed points, the group is said to act freely. When every point has a neighborhood $V$ such that $\{\gamma \in \Gamma: \gamma(V) \cap V \neq \emptyset\}$ is finite, the group is said to act properly discontinuously (see, for example, Ref. [105]). These are the restrictions on the group action which permit to avoid conical singularities and other pathologies in the quotient manifold.

Let us denote by $\tilde{H}_{n}^{N}$ the universal covering space of $H_{n}^{N}$. Then $\tilde{H}^{N}=H^{N}$ and $\tilde{H}_{n}^{N}=H_{n}^{N}$ for $n \neq 0,1$, since these spaces are already simply connected. Now we have [103]

Theorem 2 Let $M_{n}^{N}$ be a complete, connected pseudo-Riemannian manifold of constant negative curvature $\kappa$, with signature $(n, N-n)$ and dimension $N \geq 2$. Then $M_{n}^{N}$ is isometric to a quotient $\tilde{H}_{n}^{N} / \Gamma$, where $\Gamma$ is a group of isometries acting freely and properly discontinuously on $\tilde{H}_{n}^{N}$.

The Riemannian case is due to Killing and Hopf. In this case $\Gamma$ is a discrete subgroup of $O^{+}(1, N)$, namely the elements of $\Gamma$ can be parametrized by a discrete label (more exactly, the 
relative topology of $\Gamma$ in $O^{+}(1, N)$ is the discrete topology). Hence the "Clifford-Klein space form problem", to classify all manifolds with constant negative curvature, is reduced to the problem of finding all the discrete subgroups of $O^{+}(1, N)$ acting freely and properly discontinuously on $H^{N}$.

The model spaces just defined have the further property to be homogeneous. By definition, this means that for every pair of points $x, y$ there is an isometry which moves $x$ into $y$. As a consequence they are complete, i.e. there are no geodesics ending or beginning at any point. In this connection, a remarkable fact concerning the hyperbolic space $H^{N}$ is that it is the only connected and homogeneous Riemannian manifold with constant negative curvature [103]. It follows that every quotient $H^{N} / \Gamma$, with $\Gamma \neq e$, cannot be a homogeneous space and thus it has less symmetry than the covering $H^{N}$. This fact restrict somewhat their use in cosmological models [106.

Up to now we have described the global properties of hyperbolic manifolds. In order to describe explicitly the metric tensor of $H^{N}$ we introduce some known model of this space.

The hyperboloid model. Let $x_{0}, \ldots, x_{N}$ on $\mathbb{R}^{N+1}$ be the standard coordinates with metric $d s^{2}=-d x_{0}^{2}+\sum_{i=1}^{N} d x_{i}^{2}$ such that the space $H^{N}$ is the upper sheet of the $N$-dimensional hyperboloid $x_{0}^{2}-\sum_{i=1}^{N} x_{i}^{2}=a^{2}$, with the induced metric. Then $\sigma \in[0, \infty)$ and $\vec{n} \in S^{N-1}$, such that

$$
x_{0}=a \cosh \sigma, \quad \vec{x}=a \vec{n} \sinh \sigma,
$$

will define a global system of coordinates for this model of $H^{N}$. A direct calculation leads to the metric tensor and volume element in the form

$$
\begin{aligned}
& d s^{2}=a^{2}\left[d \sigma^{2}+\sinh ^{2} \sigma d \ell_{N-1}^{2}\right], \\
& d V=a^{N-1}(\sinh \sigma)^{N-1} d \sigma d \Omega_{N-1},
\end{aligned}
$$

where $d \ell_{N-1}$ and $d \Omega_{N-1}$ are the line and volume element of $S^{N-1}$ respectively. This metric is of some interest, since the coordinate $\sigma$ measures the geodesic distance of any point from the bottom of the hyperboloid, the point $x_{0}=a$. It is also obvious that the group which fixes this point is the group $O(N)$ of rotations around the $x_{0}$-axis. Thus we can regard $H^{N}$ as the coset space $O^{+}(1, N) / O(N)$.

The cylinder model. Assuming $\rho \in[0, \pi / 2)$ and $\tau \in(-\infty,+\infty)$, the global metric tensor in this model is

$$
d s^{2}=a^{2}(\cos \rho)^{-2}\left[d \tau^{2}+d \rho^{2}+\sin ^{2} \rho d \ell_{N-2}^{2}\right],
$$

where $d \ell_{N-2}$ is the line element of $S^{N-2}$. This metric is conformal to half the cylinder $\mathbb{R} \times S^{N-1}$, with the product metric. In 2 -dimensions, this is the strip $-\pi / 2<\rho<\pi / 2,-\infty<\tau<\infty$ with metric 96 .

$$
d s^{2}=a^{2}(\cos \rho)^{-2}\left[d \tau^{2}+d \rho^{2}\right],
$$

which still provides another model for $H^{2}$. The coordinate $\tau$ can be directly interpreted as imaginary time, because under Wick rotation $\tau \rightarrow-i$, the metric (3.20) goes over to the anti-de Sitter metric, which is in fact conformal to a region of the Einstein static universe. 
The ball model. We shall seldom make use of this model but we give it for completeness. We denote by $B^{N}$ the ball in $\mathbb{R}^{N}$ of radius $2 a$. We set

$$
u=\frac{2 a \sinh \sigma}{\cosh \sigma+1}, \quad \quad u^{2}<4 a^{2} .
$$

In terms of $u$, the metric tensor Eq. (3.19), takes the conformally flat form

$$
d s^{2}=\left(1-\frac{u^{2}}{4 a^{2}}\right)^{-2}\left[d u^{2}+u^{2} d \ell_{N-1}^{2}\right] .
$$

The discovery of this metric and the observation that it has constant curvature, was one of Riemann great contributions. The geodesics are the circles or lines that meet the boundary of the disc orthogonally.

The Poincaré half-space model. There is a conformal map from the ball $B^{N}$ of radius $2 a$ in $\mathbb{R}^{N}$ onto the half space $x_{N}>0$ [105]. For convenience, we rename $x_{N}=r$. Poincaré used this map to transform the ball metric into the half-space metric

$$
d s^{2}=\frac{a^{2}}{r^{2}}\left(d x_{1}^{2}+\ldots+d x_{N-1}^{2}+d r^{2}\right) .
$$

The distance $d(X, Y)$ between two points $X=\left(x^{1}, \ldots, x^{N-1}, r\right)$ and $Y=\left(y^{1}, \ldots, y^{N-1}, s\right)$ in this model is implicitly given by

$$
\cosh \left[\frac{d(X, Y)}{a}\right]-1=\frac{|X-Y|^{2}}{2 r s},
$$

where $|X-Y|$ is the Euclidean distance. The formula is easily found, first for $X=(0, \ldots, 0, r)$ and $Y=(0, \ldots, 0, s)$ and then for any pair of points, using the transitivity of isometries, since both sides of Eq. (3.21) are invariants. The geodesics of this metric are the circles or straight lines that meet the boundary $r=0$ orthogonally. It is geometrically obvious that for every pair of points there is a unique minimizing geodesic connecting them.

This latter is the model we shall employ in discussing the Selberg trace formula. Of course, the curvature constant $\kappa=-1 / a^{2}$ in all the four models.

\subsubsection{The Laplace operator and the density of states}

In the present subsection, we normalize the curvature to $\kappa=-1$ for the sake of simplicity. The first step towards the Selberg trace formula is to compute the spectral decomposition of the Laplace operator on $H^{N}$. This is equivalent to find the density of states, since we expect this operator to have a continuous spectrum. This density gives the simplest kind of trace formula, like

$$
\operatorname{Tr}(h(\Delta))=\int_{S} h(r) \Phi_{N}(r) d r
$$

where the density $\Phi_{N}(r)$ is defined over the spectrum $S$ of $\Delta$ and for functions $h(r)$ for which the trace and the integral exist. Here it is convenient to use the hyperboloid model of $H^{N}$. A simple computation with the metric (3.19) gives the Laplace operator

$$
\Delta=\frac{\partial^{2}}{\partial \sigma^{2}}+(N-1) \operatorname{coth} \sigma \frac{\partial}{\partial \sigma}+(\sinh \sigma)^{-2} \Delta_{S^{N-1}}
$$

where the last term denotes the Laplacian on the unit sphere $S^{N-1}$. The eigenvalues equation is $-\Delta \phi=\lambda \phi$. Note that if we replace $\lambda$ with $\lambda+m^{2}$ we can also include the massive operator 
in our calculations. Clearly the eigenfunctions have the form $\phi=f_{\lambda}(\sigma) Y_{l m}$ where $Y_{l m}$ are the spherical harmonics on $S^{N-1}$ and for simplicity we leave understood the dependence of $f_{\lambda}$ on $l, l=0,1,2 \ldots$ and $m=\left(m_{1}, \ldots, m_{N-2}\right)$ being the set of angular momentum quantum numbers. For these harmonics we have

$$
\Delta_{S^{N-1}} Y_{l m}=-l(l+N-2) Y_{l m} .
$$

Thus, the radial wave functions will satisfy the ordinary differential equation

$$
f_{\lambda}^{\prime \prime}+(N-1) \operatorname{coth} \sigma f_{\lambda}^{\prime}+\left[\lambda-\frac{l(l+N-2)^{2}}{\sinh ^{2} \sigma}\right] f_{\lambda}=0 .
$$

If the zero angular momentum wave functions are normalized so that $f_{\lambda}(0)=1$, their density is known to mathematicians as the Harish-Chandra or Plancherel measure.

We continue by transforming the radial wave equation into a known form. On setting $\rho_{N}=(N-1) / 2, \mu_{l}=1-l-N / 2, r=\sqrt{\left(\lambda-\rho_{N}^{2}\right)}$, defining $v_{\lambda}(\sigma)$ by

$$
f_{\lambda}(\sigma)=(\sinh \sigma)^{1-N / 2} v_{\lambda}(\sigma)
$$

and performing the change of variable $x=\cosh \sigma$, we get the differential equation of associated Legendre functions

$$
\frac{d}{d x}\left[\left(1-x^{2}\right) \frac{d v_{\lambda}}{d x}\right]+\left[\nu(\nu+1)-\frac{\mu_{l}^{2}}{1-x^{2}}\right] v_{\lambda}=0,
$$

with parameters $\nu=-1 / 2 \pm i r$. The invariant measure defining the scalar product between eigenfunctions is

$$
\left(f_{\lambda}, f_{\lambda^{\prime}}\right)=\Omega_{N-1} \int_{0}^{\infty} f_{\lambda}^{*} f_{\lambda^{\prime}}(\sinh \sigma)^{N-1} d \sigma=\Omega_{N-1} \int_{1}^{\infty} v_{\lambda}^{*} v_{\lambda^{\prime}} d x
$$

where $\Omega_{N-1}$ is the volume of the $N-1$ dimensional sphere.

The only bounded solutions of Eq. (3.25) are the associated Legendre functions of the first kind and denoted by $P_{\nu}^{\mu}(x)$ [107. Hence, the radial wave functions are

$$
f_{\lambda}(\sigma)=\Gamma\left(\frac{N}{2}\right)\left(\frac{\sinh \sigma}{2}\right)^{1-N / 2} P_{-1 / 2+i r}^{\mu}(\cosh \sigma),
$$

where the multiplicative constant has been chosen in order to satisfy the normalization condition $f_{\lambda}(0)=1$.

The density of states is determined from the knowledge of the radial wave functions as follows. One chooses a variable $r$ parametrizing the continuum spectrum so that $\lambda=\lambda(r)$ and computes the scalar product

$$
\left(\phi_{\lambda}, \phi_{\lambda^{\prime}}\right)=\frac{1}{\Phi_{N}(r)} \delta\left(r-r^{\prime}\right),
$$

which defines the density of states $\Phi_{N}(r)$. Let us choose $r=\left(\lambda-\rho_{N}^{2}\right)^{1 / 2}$ as a label for the continuum states. The Legendre functions are given in terms of hypergeometric functions. For $\operatorname{Re} z>1$ and $|1-z|<2$ they are

$$
P_{\nu}^{\mu}(z)=\frac{1}{\Gamma(1-\mu)}\left(\frac{z+1}{z-1}\right)^{\mu / 2} F(-\nu, \nu+1 ; 1-\mu ;(1-z) / 2) .
$$

These are analytic throughout the complex plane, with a cut along the real axis from $-\infty$ up to 1 . The asymptotic behaviour for $|z| \gg 1$ is

$$
P_{\nu}^{\mu}(z) \approx \frac{2^{\nu} \Gamma(\nu+1 / 2)}{\pi^{1 / 2} \Gamma(\nu-\mu+1)} z^{\nu}+\frac{\Gamma(-\nu-1 / 2)}{2^{\nu+1} \pi^{1 / 2} \Gamma(-\nu-\mu)} z^{-\nu-1},
$$


from which we obtain the asymptotic behaviour of the eigenfunctions

$$
f_{\lambda}(\sigma) \simeq \frac{2^{N} \Gamma(N / 2) \Gamma(i r)}{4 \pi^{1 / 2} \Gamma\left(\rho_{N}+i r\right)} e^{-\rho_{N} \sigma+i r \sigma}+\text { c.c. } .
$$

As a result, the radial functions will remain bounded at infinity provided the parameter $r$ were real, which is equivalent to the condition $\lambda \geq \rho_{N}^{2}$. Thus, the spectrum of the Laplacian has a gap which is determined by the curvature and depends on $N$, although the excitations of the corresponding wave operator on $\mathbb{R} \times H^{N}$ still propagate on the light cone. Due to this gap, the Green functions of $\Delta$ on $H^{N}$ are exponentially decreasing at infinity. As a consequence, negative constant curvature provides a natural infrared cut-off [108] for interacting boson field theories.

Now we come to compute the scalar product between two radial eigenfunctions. Using the fact that the product of two eigenfunctions is the derivative of the their Wronskian $W[\cdot, \cdot]$, we get

$$
\left(f_{\lambda}, f_{\lambda^{\prime}}\right)=\frac{2^{N-1} \pi^{\frac{N}{2}} \Gamma\left(\frac{N}{2}\right)}{\nu^{*}\left(\nu^{*}+1\right)-\nu^{\prime}\left(\nu^{\prime}+1\right)} \lim _{x \rightarrow \infty}\left(1-x^{2}\right) W\left[P_{\nu^{*}}^{\mu}(x), P_{\nu^{\prime}}^{\mu}(x)\right]
$$

where the limit is taken in the sense of distributions. Now we can use the asymptotic form of the Legendre functions to compute the limit. In this way Eq. (3.31) reduces to Eq. (3.28) with the density of states given by

$$
\Phi_{N}(r)=\frac{2}{(4 \pi)^{N / 2} \Gamma(N / 2)} \frac{\left|\Gamma\left(i r+l+\rho_{N}\right)\right|^{2}}{|\Gamma(i r)|^{2}} .
$$

When $l=0$ we have the density of zero angular momentum radial functions and Eq. (3.32) reduces to the Harish-Chandra measure.

We stress that $d n=\Phi_{N}(r) d r$ is the number of states per unit volume in the range $d r$. Hence the scalar $\zeta$-function per unit volume reads

$$
\tilde{\zeta}\left(s \mid L_{N}\right)=a^{2 s-N} \int_{0}^{\infty}\left[r^{2}+(a \alpha)^{2}\right]^{-s} \Phi_{N}(r) d r .
$$

It is independent from $X$ because $H^{N}$ is homogeneous. As in Sec. 3.2, $L_{N}=-\Delta_{N}+\alpha^{2}+\kappa \varrho_{N}^{2}$, but now $\alpha \geq 0$ and $\kappa<0\left(a^{2}=|\kappa|^{-1}\right)$. The particular cases $N=3$ and $N=4$ deserve some attention. For these two cases, we have respectively

$$
\begin{aligned}
\tilde{\zeta}\left(s \mid L_{3}\right)= & \frac{\alpha^{3-2 s}}{(4 \pi)^{3 / 2}} \frac{\Gamma(s-3 / 2)}{\Gamma(s)}, \\
\tilde{\zeta}\left(s \mid L_{4}\right)= & \frac{1}{16 \pi^{2}}\left[\frac{\alpha^{4-2 s}}{(s-1)(s-2)}+\frac{\alpha^{2-2 s}}{4 a^{2}(s-1)}\right] \\
& \quad-\frac{a^{2 s-4}}{4 \pi^{2}} \int_{0}^{\infty}\left[r^{2}+(a \alpha)^{2}\right]^{-s} \frac{r\left(r^{2}+\frac{1}{4}\right)}{e^{2 \pi r}+1} d r,
\end{aligned}
$$

where the meromorphic structure can be immediately read off. The quantity $\zeta^{\prime}\left(0 \mid L_{4}\right)$ gives the one-loop functional determinant for a scalar field on anti-de Sitter space-time, while $\zeta\left(s \mid L_{4}\right)$ itself is related to the free energy density on such a space-time. 


\subsubsection{The Dirac operator and the density of states}

Here we consider the Dirac-like equations on $H^{N}$. Let $N$ be even. The $N$-dimensional Clifford algebra has only one complex, irreducible representation in the $2^{N / 2}$-dimensional spinor space. These spinors are reducible with respect to the even subalgebra (generated by products of an even number of Dirac matrices) and split in a pair of $2^{N / 2-1}$-component irreducible Weyl spinors.

The Dirac eigenvalue equation is

$$
i \not \nabla \psi-m \gamma_{0} \psi=-\omega \psi,
$$

where $m$ is a mass parameter and $i \not \nabla$ is the Dirac operator on $H^{N}$. It can be regarded as the full time-dependent Dirac equation on $\mathbb{R} \times H^{N}$ restricted on time-harmonic fields. When we use the hyperboloid model of $H^{N}$, the submanifolds with $r$ a constant are a family of spheres $S^{N-1}$ covering the space. The covariant derivative of a spinor field on $H^{N}$ can be decomposed into a radial part plus the covariant derivative along the unit $(N-1)$-sphere. Making this decomposition in a Dirac-like representation of gamma matrices, the equation takes the form of a coupled system

$$
\begin{aligned}
& i \gamma_{1}\left(\partial_{\sigma}+\rho_{N} \operatorname{coth} \sigma\right) \psi_{1}+\frac{1}{\sinh \sigma} i \not_{s} \psi_{1}=-(\omega+m) \psi_{2}, \\
& i \gamma_{1}\left(\partial_{\sigma}+\rho_{N} \operatorname{coth} \sigma\right) \psi_{2}+\frac{1}{\sinh \sigma} i \not_{s} \psi_{2}=-(\omega-m) \psi_{1},
\end{aligned}
$$

where $\psi_{1,2}$ are the $2^{N / 2-1}$-components Weyl spinors into which the original representation decomposes and $i \nabla_{s}$ is the Dirac operator on $S^{N-1}$. The spinors $\psi_{1,2}$ transform irreducibly under $S O(N)$ so that we can put $\psi_{1,2}=f_{1,2}(\sigma) \chi_{1,2}$, where $\chi_{1,2}$ are spinors on $S^{N-1}$. Separation of variables is achieved on requiring $i \nabla_{s} \chi_{1}=i \lambda \chi_{2}$ and $\gamma_{1} \chi_{1}=\chi_{2}$. Since $\gamma_{1} \gamma_{1}=1$ and $\left\{\gamma_{1}, i \not_{s}\right\}=0$ we also have $i \not_{s} \chi_{2}=-i \lambda \chi_{1}$ and $\gamma_{1} \chi_{2}=\chi_{1}$.

The eigenvalues of the Dirac operator on $S^{N-1}$ are known to be $\lambda= \pm\left(l+\rho_{N}\right), l=0,1,2, \ldots$ [109]. Hence, we obtain two radial equations which are equivalent to the $2^{\text {nd }}$-order system

$$
\begin{aligned}
& \frac{d}{d z}\left[\left(z^{2}-1\right) \frac{d \phi_{1}}{d z}\right]+\left[r^{2}+\rho_{N}^{2}-\frac{N}{2}-\frac{\left(\lambda^{2}-\rho_{N}^{2}+\rho_{N}\right)+\lambda z+\left(\rho_{N}-\frac{1}{2}\right)^{2} z^{2}}{z^{2}-1}\right] \phi_{1}=0, \\
& \frac{d}{d z}\left[\left(z^{2}-1\right) \frac{d \phi_{2}}{d z}\right]+\left[r^{2}+\rho_{N}^{2}-\frac{N}{2}-\frac{\left(\lambda^{2}-\rho_{N}^{2}+\rho_{N}\right)-\lambda z+\left(\rho_{N}-\frac{1}{2}\right)^{2} z^{2}}{z^{2}-1}\right] \phi_{2}=0,
\end{aligned}
$$

where we have defined $z=\cosh \sigma, \phi_{1,2}=(\sinh \sigma)^{N / 2-1} f_{1,2}(\sigma)$ and $r^{2}=\omega^{2}-m^{2}$.

For odd $N$, there are two $2^{(N-1) / 2}$-components irreducible spinors. Since we are regarding the Dirac equation on $H^{N}$ as the restriction of the Dirac equation on $\mathbb{R} \times H^{N}$ on time-harmonic fields, we must take into account both spinors. We can say that they are the Weyl spinors of the unphysical space-time $\mathbb{R} \times H^{N}$. Both belong to the same irreducible representation of $S O(N)$, thus we can separate variables as above and we obtain exactly the same equations as (3.33), (3.34) or (3.35) and (3.36). Of course, there is nothing wrong to consider the Dirac equation directly on $H^{N}$ without thinking about it as the spatial section of an unphysical space-time. However, for $N=3$ we do have a physical space-time. Alternatively, one may consider the spinor Laplacian as the relevant operator. This point of view is taken in Ref. [110]. We can set $m=0$, then the equations decouples into pairs of equivalent Dirac equations on $H^{N}$ and we should recover the results of Ref. [110]. 
The solutions of Eqs. (3.35) and (3.36) are given in terms of hypergeometric functions as follows. Let $f_{1,2}^{ \pm}(r)$ the solutions with $\lambda= \pm\left(l+\rho_{N}\right)$ and set $\alpha=l+N / 2+i r$. We find

$$
\begin{aligned}
& f_{1}^{+}(\sigma)=A(1+z)^{l / 2}(z-1)^{(l+1) / 2} F\left(\alpha, \alpha^{*} ; l+\rho_{N}+\frac{3}{2} ; \frac{1-z}{2}\right), \\
& f_{2}^{+}(\sigma)=B(1+z)^{(l+1) / 2}(z-1)^{l / 2} F\left(\alpha, \alpha^{*} ; l+\rho_{N}+\frac{1}{2} ; \frac{1-z}{2}\right), \\
& f_{1}^{-}(\sigma)=C(1+z)^{(l+1) / 2}(z-1)^{l / 2} F\left(\alpha, \alpha^{*} ; l+\rho_{N}+\frac{1}{2} ; \frac{1-z}{2}\right), \\
& f_{2}^{-}(\sigma)=D(1+z)^{l / 2}(z-1)^{(l+1) / 2} F\left(\alpha, \alpha^{*} ; l+\rho_{N}+\frac{3}{2} ; \frac{1-z}{2}\right) .
\end{aligned}
$$

The ratios $A / B=i(\omega+m)(l+N / 2)^{-1}$ and $D / C=i(\omega-m)(l+N / 2)^{-1}$ are determined by demanding that the solutions of the second order system satisfy the first order Dirac equation. Next we choose $B=(4 \omega)^{-1 / 2}(\omega-m)^{1 / 2}$ and $C=(4 \omega)^{-1 / 2}(\omega+m)^{1 / 2}$. Then the asymptotic behaviour for $r \rightarrow \infty$ of the radial solutions takes the symmetric form

$$
\begin{aligned}
& f_{1}^{ \pm}(\sigma) \simeq\left(\frac{\omega+m}{2 \omega}\right)^{1 / 2} \mathcal{C}(r) e^{-\rho_{N} \sigma+i r \sigma}+\text { c.c. } \\
& f_{2}^{ \pm}(\sigma) \simeq\left(\frac{\omega-m}{2 \omega}\right)^{1 / 2} \mathcal{C}(r) e^{-\rho_{N} \sigma+i r \sigma}+\text { c.c. }
\end{aligned}
$$

where we introduced the meromorphic function

$$
\mathcal{C}(r)=\frac{2^{N-1+l-2 i r} \Gamma(l+N / 2) \Gamma(2 i r)}{\Gamma(N / 2+l+i r) \Gamma(i r)} .
$$

One can see that the solutions remain bounded at infinity if $r$ is real. Hence, the spectrum of the Dirac operator on $H^{N}$ is $|\omega| \geq m$. For $m=0$ it extends over the entire real axis. Thus, we reach the conclusion that unlike the scalar case, there is no gap for fermions on $H^{N}$. Nevertheless, the solutions are exponentially vanishing at infinity.

The function (3.37) for $l=0$ determines the Plancherel measure or the density of states with zero angular momentum, which has to be used in the evaluation of the spinor $\zeta$-function. We define the density $\mu_{N}(r)$ so that the spinor $\zeta$-function per unit volume is given by

$$
\tilde{\zeta}\left(s \mid D_{N}\right)=: \operatorname{tr}\left(-\not^{2}+m^{2}\right)^{-s}(X, X)=2^{\left[\frac{N}{2}\right]} \int_{0}^{\infty}\left(r^{2}+m^{2}\right)^{-s} \mu_{N}(r) d r .
$$

Here $D_{N}$ represents the massive Dirac operator on $H^{N},[N / 2]$ denotes the integer part of $N / 2$ and the trace is over the spinor indices. Again, the $X$-independence comes from the homogeneity of $H^{N}$.

Assuming that the spherical spinors are already normalized and using $r$ as a label for the continuous spectrum, we obtain the density

$$
\mu_{N}(r)=\frac{\Gamma(N / 2) 2^{N-3}}{\pi^{N / 2+1}}|\mathcal{C}(r)|^{-2}
$$

which, apart from a slightly different definition of the measure, agrees with results given in Ref. [110], where it has been also noted that in the even case, the poles and residues of the density determine the spectrum and degeneracies of the spinor Laplacian on the sphere $S^{N}$. For odd $N$ the density is analytic.

In 3-dimensions the $\zeta$-function density becomes

$$
\tilde{\zeta}\left(s \mid D_{3}\right)=\frac{2 m^{3-2 s}}{(4 \pi)^{3 / 2}} \frac{\Gamma(s-3 / 2)}{\Gamma(s)}\left(1+\frac{2 s-3}{(2 a m)^{2}}\right) .
$$


Note that the zero mass limit is not uniform with respect to $s$, unlike the scalar case. The reason is the absence of the gap in the spectrum for fermions. In 4-dimensions it takes the form

$$
\begin{aligned}
\tilde{\zeta}\left(s \mid D_{4}\right)=\frac{1}{4 \pi^{2}}\left[\frac{m^{4-2 s}}{(s-1)(s-2)}+\frac{m^{2-2 s}}{a^{2}(s-1)}\right] \\
\quad+\frac{a^{2 s-4}}{\pi^{2}} \int_{0}^{\infty}\left[r^{2}+(a m)^{2}\right]^{-s} \frac{r\left(r^{2}+1\right)}{e^{2 \pi r}-1} d r .
\end{aligned}
$$

Again, this function determines the one-loop spinor determinant on anti-de Sitter space-time [11] as well as the fermion free energy density.

\subsection{Compact hyperbolic manifolds}

We have seen that every complete, connected hyperbolic Riemannian manifold $\mathcal{M}$ is a quotient of $H^{N}$ by a discontinuous group $\Gamma$ of isometries. There is a huge number of such manifolds. In the case $N=3$, their relevance as spatial sections for Robertson-Walker-Freedman cosmologies has been discussed in Ref. [106]. One interesting feature of these spaces lies in the mismatch between non trivial topology and negative curvature, which permits a variety of situations. In particular, they can describe a finite universe that expands forever, contrary to the belief that a locally hyperbolic universe must be infinite. To have some ideas of the spaces $H^{N} / \Gamma$, we start with an elementary classification of the isometries of $H^{N}$ since this is also the second important step towards the Selberg trace formula.

We write a point in $H^{N}$ as $X=(x, r)$, where $x=\left(x_{1}, \ldots, x_{N-1}\right)$ belongs to the boundary $\partial H^{N}$ (the plane $r=0$ ) and $r>0$, namely we are considering the half-space model of $H^{N}$. It is understood that $\{\infty\}$ is considered to be a point of $\partial H^{N}$, that is $\partial H^{N}=\mathbb{R}^{N-1} \cup\{\infty\}$. This is done in order to make the inversion well defined at the origin.

Let $G_{N}$ be the group of motions of $\mathbb{R}^{N} \cup\{\infty\}$ which is generated by the following transformations:

(i) translations: $(x, r) \rightarrow(x+y, r), \infty \rightarrow \infty$,

(ii) rotations: $(x, r) \rightarrow(\Lambda x, r), \infty \rightarrow \infty(\Lambda \in O(N-1))$,

(iii) dilatations: $X \rightarrow \lambda X, \infty \rightarrow \infty(\lambda>0)$,

(iv) inversions: $X \rightarrow a+(X-a) /|X-a|^{2}, a \rightarrow \infty, \infty \rightarrow a\left(a \in \partial H^{N}\right)$.

Three elementary facts about $G_{N}$ are noteworthy [105, 112]:

(a) $G_{N}$ preserves both $H^{N}$ and $\partial H^{N}$. As a group of motions of $\partial H^{N}$ (obtained setting $r=0$ in (i) through (iv)) it is the conformal group of the flat metric. The dimension is $N(N+1) / 2$.

(b) Regarding $\partial H^{N}$ as the plane $r=0$ in $\mathbb{R}^{N} \cup\{\infty\}$, there is a natural inclusion $G_{N} \subset G_{N+1}$, the full conformal group of $\mathbb{R}^{N} \cup\{\infty\}$.

(c) $G_{N}$ is the group of all isometries of $H^{N}$.

The first half of statement (a) is trivial as well as the statement (b). It is a simple exercise to show that elements of $G_{N}$ act as isometries of the Poincaré metric. Since $N(N+1) / 2$ is the maximal dimension permitted by a group of isometries, the statement (c) follows. As a consequence, $G_{N}$ is isomorphic to the Lorentz group $O^{+}(1, N)$. By inspection of the elements of $G_{N}$, it is clear that every $\gamma \in G_{N}$ has at least one fixed point in $H^{N} \cup \partial H^{N}$. We say that $\gamma$ is elliptic if $\gamma$ has at least one fixed point in $H^{N}$ (rotations and inversions are example of elliptic elements). If there is only one fixed point and it lies in $\partial H^{N}$, then $\gamma$ is parabolic (translations 
are examples of parabolic elements). Otherwise $\gamma$ is loxodromic (dilatations are examples of loxodromic elements). If there are three or more fixed points in $\partial H^{N}$, then $\gamma$ must be elliptic [112] (i.e. it fixes some point in $H^{N}$ ). Thus every loxodromic element has exactly two fixed points in $\partial H^{N}$.

Since fixed points produce metric singularities in the quotient manifold, we have that $H^{N} / \Gamma$ is a singularity-free Riemannian space only if $\Gamma$ does not contain elliptic elements. Now we can use the explicit form of the generators to find a canonical form for the various isometries. We recall that two elements $\gamma$ and $\gamma^{\prime}$ in a group are conjugate if $\gamma=g \gamma^{\prime} g^{-1}$ for some $g$. Conjugation is an equivalence relation so that the group is a disjoint union of conjugacy classes.

First let $\gamma$ be elliptic and $X$ a fixed point. We can move $X$ to the point $(0,1)$ with an isometry $g$. Thus $g \gamma g^{-1}$ fixes $(0,1)$. Since translations and dilatations do not fix $(0,1), \gamma$ is conjugate to a product of inversions and a rotation around the vertical line $\{(0, r): r>0\}$. For future references, we call this line $H^{1}$. A better description of elliptic elements may be the following: we regard $\gamma$ as acting in $B^{N}$ with fixed point the origin. Since the ball metric is radial, it is clear that $\gamma \in O(N)$ is a rotation or a reflection around the origin. But there is a conformal transformation $q \in G_{N+1}$ which sends $H^{N}$ onto $B^{N}$ and the point $(0,1)$ into the origin. Thus, every elliptic element is conjugate in $G_{N+1}$ to a rotation or reflection around the origin. This fact has an interesting consequence in $H^{3}$. A rotation in 3-dimensions always has an eigenvalue equal to 1 or -1 , i.e. an axis of rotation. This will be a radial geodesic in the ball $B^{3}$. Going back to $H^{3}$, we conclude that every elliptic element is a rotation around a unique invariant axis, namely around a circle or line that meets $\partial H^{N}$ orthogonally.

Now we suppose $\gamma$ is a loxodromic element with fixed points $x, y$ and $\ell$ is the geodesic in $H^{N}$ joining $x$ to $y$. This is called the axis of $\gamma$. Clearly $\gamma(\ell)=\ell$, since $\gamma$ maps geodesics into geodesics and there is only one geodesic joining $x$ to $y$. We can map the axis to the vertical line $H^{1}$ with an isometry $g$. Thus $g \gamma g^{-1}$ leaves $H^{1}$ invariant with fixed points 0 and $\infty$. Translations and inversions do not have this property, thus, $\gamma$ must be conjugate to a rotation around $H^{1}$ followed by a dilatation. If the rotation is trivial, i.e. if $\gamma$ is conjugate to a dilatation, we say that $\gamma$ is a hyperbolic isometry. In the Lorentz group of the hyperboloid model, such a $\gamma$ is conjugate to a Lorentz boost.

Finally, if $\gamma$ is parabolic, we can move the fixed point at $\infty$ with an isometry $g$. Hence, $g \gamma g^{-1}$ is an isometry fixing only $\infty$. Since the inversion and dilatations do not have this property, $\gamma$ must be conjugate to a rotation followed by a translation, i.e. $g \gamma g^{-1}(X)=\Lambda(X)+a$. Actually, one can choose the point $a$ and the matrix $\Lambda$ such that $\Lambda(a)=a$. This is the normal form of a parabolic element.

Clearly, conjugation preserves the isometry types so that the conjugacy classes are classified as elliptic, loxodromic and parabolic classes.

Now we suppose that $\mathcal{M}=H^{N} / \Gamma$ is a compact manifold. The action of $\Gamma$ must be free, otherwise $\mathcal{M}$ is not a manifold, in general. Then $\Gamma$ is torsion-free, which means it does not contain elements of finite order [113]. Moreover $\Gamma$ is discontinuous and thus it must be a discrete subgroup of $G_{N}$ [112].

What are the consequences of these facts? The most important for us is that every element of $\Gamma$ must be a loxodromic isometry. Thus $\Gamma$ will not contain any parabolic element. Such a group is called co-compact. Very briefly, the argument runs as follows. Consider the distance function $d(X, \gamma X)$ on $H^{N}$ where $\gamma \in \Gamma$. Any arc joining $X$ and $\gamma X$ projects to a closed homotopically non trivial loop on $\mathcal{M}$. For, if it were possible to shrink the loop continuously to a point, it would be possible to move $\gamma$ continuously into the identity without exit $\Gamma$. This cannot be done because $\Gamma$ is discrete. Thus the function is strictly positive and there must be an $X_{0}$ at which $d\left(X_{0}, \gamma X_{0}\right)$ is minimum. The reason is that the distance function is continuous and $\mathcal{M}$ is compact. It follows that the minimal geodesic $J_{\gamma}$ joining $X_{0}$ and $\gamma X_{0}$ is invariant under $\gamma$, since any other geodesic will have greater length. $J_{\gamma}$ will intersect $\partial H^{N}$ in two points which are fixed points of $\gamma$ and 
so $\gamma$ will be a loxodromic isometry. Recalling the normal form of such an isometry as given above, we see that if $\mathcal{M}$ is compact, then every $\gamma \in \Gamma$ is conjugate in $G_{N}$ to a transformation of the form $D \Lambda$, where $D:(x, r) \rightarrow(\lambda x, \lambda r)$ is a dilatation and $\Lambda$ is a rotation acting on $x$. The invariant geodesic $J_{\gamma}$ is called the axis of $\gamma$. Moreover, if $\gamma_{1}$ and $\gamma_{2}$ commute, they share a common axis. A second fact is that any non trivial abelian subgroup of $\Gamma$ must be infinite cyclic. In particular, the centralizer $\mathcal{C}_{\gamma}$ of any non trivial element $\gamma$ must be infinite cyclic. $\mathcal{C}_{\gamma}$ is the set of elements $g \in \Gamma$ which commute with $\gamma$. The third fact is the existence of a convex polyhedron $\mathcal{F}$ in $H^{N}$ such that [112] (a) the hyperbolic volume of $\mathcal{F}$ is finite, (b) $\gamma(\mathcal{F}) \cap \mathcal{F}=\emptyset$ for all non trivial $\gamma \in \Gamma$, (c) the translates of $\mathcal{F}$ tessellate $H^{N}$, that is $\bigcup_{\gamma \in \Gamma} \gamma(\mathcal{F})=H^{N}$, (d) for every face $s$ of $\mathcal{F}$ there is a face $s^{\prime}$ and an element $\gamma_{s} \in \Gamma$ with $\gamma_{s}(s)=s^{\prime}$ and $\gamma_{s}\left(s^{\prime}\right)=s$. That is the faces of $\mathcal{F}$ are paired by elements of $\Gamma$. Note that the faces of $\mathcal{F}$ are portions of $(N-1)$-dimensional spheres or planes orthogonal to $\partial H^{N}$. Such a polyhedron is called a fundamental domain for $\Gamma$. Conversely, the Poincaré polyhedron theorem roughly states that given a polyhedron with the above properties (plus additional technical assumptions), the group generated by the face pairing transformations is discrete with fundamental domain being the given polyhedron [112]. As a result, these manifolds can be obtained by identifying the faces of a finite volume polyhedron in a suitable way.

\subsubsection{The Selberg trace formula for scalar fields}

We begin by stating the trace formula and then we try to illustrate it, first restricting ourselves to a strictly hyperbolic group $\Gamma$ such that $H^{N} / \Gamma$ is compact with fundamental domain $\mathcal{F}_{N}$ and the volume $\Omega\left(\mathcal{F}_{N}\right)$ of $\mathcal{M}$ is finite.

Let $h(r)$ be an even and holomorphic function in a strip of width larger than $N-1$ about the real axis, such that $h(r)=O\left(r^{-(N+\varepsilon)}\right)$ uniformly in the strip as $r \rightarrow \infty$. Let $\chi(\gamma): \Gamma \rightarrow S^{1}$

be a character of $\Gamma$. Let us denote by $A_{\Gamma, \chi}$ the non negative, unbounded, self-adjoint extension of the Laplace operator $\Delta_{\Gamma}$ acting in the Hilbert space $\mathcal{H}(\Gamma, \chi)$ of square integrable automorphic functions on a fundamental domain $\mathcal{F}_{N}$, relative to the invariant Riemannian measure. A scalar function $\phi$ is called automorphic (relative to $\Gamma$ and $\chi$ ) if for any $\gamma \in \Gamma$ and $x \in H^{N}$ the condition $\phi(\gamma x)=\chi(\gamma) \phi(x)$ holds. We will refer automorphic functions with $\chi \neq 1$ as twisted scalar fields. For $\Gamma$ co-compact, the operator $A(\Gamma, \chi)$ has pure discrete spectrum with isolated eigenvalues $\lambda_{j}$, $j=0,1,2, . . \infty$ of finite multiplicity. With these assumptions, the Selberg trace formula holds (see Ref .114])

$$
\sum_{j=0}^{\infty} h\left(r_{j}\right)=\Omega\left(\mathcal{F}_{N}\right) \int_{0}^{\infty} h(r) \Phi_{N}(r) d r+\sum_{\{\gamma\}} \sum_{n=1}^{\infty} \frac{\chi^{n}(\gamma) l_{\gamma}}{S_{N}\left(n ; l_{\gamma}\right)} \hat{h}\left(n l_{\gamma}\right) .
$$

Above, each number $r_{j}$ is that root of $r_{j}^{2}=\left(\lambda_{j}-\rho_{N}^{2}\right)$ having positive imaginary part and the sum over $j$ includes the eigenvalues multiplicity. The symbols $S_{N}\left(n ; l_{\gamma}\right),\{\gamma\}, l_{\gamma}$ will be defined soon, while the function $\hat{h}(p)$ is the Fourier transform of $h(r)$ defined by

$$
\hat{h}(p)=\frac{1}{2 \pi} \int_{-\infty}^{\infty} e^{i p r} h(r) d r
$$

and $\Phi_{N}(r)$ is the density of states we computed in the previous section. In particular we have

$$
\Phi_{2}(r)=\frac{r}{2 \pi} \tanh \pi r, \quad \Phi_{3}(r)=\frac{r^{2}}{2 \pi^{2}}
$$

and $\Phi_{N}$ satisfies the recurrence relation

$$
\Phi_{N+2}(r)=\frac{\rho_{N}^{2}+r^{2}}{2 \pi N} \Phi_{N}(r),
$$


which permits to obtain any $\Phi_{N}$ starting from $\Phi_{2}$ or $\Phi_{3}$ according to whether $N$ is even or odd.

The left hand side of Eq. (3.38) is a result of functional calculus. To see this, let we define $\tilde{h}(\lambda)=h(r)$ for $\lambda=r^{2}+\rho_{N}^{2}$. Then the left hand side is the operator trace of $\tilde{h}\left(A_{\Gamma, \chi}\right)$, because the Laplacian is a self-adjoint operator, so the trace must be given by the sum of $\tilde{h}(\lambda)$ over the eigenvalues. Thus the left member of the trace formula is determined by the spectrum of the Laplace operator.

The twist condition is the analog on $H^{N} / \Gamma$ of the "periodicity up to a phase" for fields on the torus $\mathbb{R}^{N} / T$, where $T$ is a discrete group of translations in $\mathbb{R}^{N}$. Hence, the only things that need an explanation are the integral and the double series on the right hand side of Eq. (3.38). We give two descriptions and a partial interpretation of them.

The group-theoretic description. We recall that every element $\gamma^{\prime} \in \Gamma$ is a loxodromic isometry and its centralizer is infinite cyclic. Thus every element commuting with $\gamma^{\prime}$, and $\gamma^{\prime}$ itself, will be the power of a generator $\gamma$, namely $\gamma^{\prime}=\gamma^{n}$, for some $n$. This element $\gamma$ is called primitive and $\{\gamma\}$ denotes the primitive conjugacy class determined by $\gamma$, i.e. the set of all elements in $\Gamma$ of the form $\gamma^{\prime \prime}=g \gamma g^{-1}$. Let us denote the trivial class by $\{e\}$ and non trivial ones by $\{\gamma\}$. It is simple to see that $\gamma^{n}$ is not conjugate to $\gamma$ if $n>1$. This means that for any non trivial primitive class, we have infinitely many other distinct classes determined by the positive powers of $\gamma$. Now, the double series in Eq. (3.38) is the sum over all the powers of any selected non trivial primitive class followed by the sum over all the non trivial primitive classes, in order to include all possible elements of $\Gamma$. We have also learned that every $\gamma$ is conjugate to the product of a dilatation $D_{\gamma}:(x, r) \rightarrow\left(N_{\gamma} x, N_{\gamma} r\right)$ with $N_{\gamma}>1$ and a rotation $\Lambda_{\gamma} \in O(N-1)$ acting on $x$. The dilatation factor $N_{\gamma}$ is called the norm of $\gamma$ and $l_{\gamma}=\ln N_{\gamma}$ by definition. The factor $S_{N}\left(n ; l_{\gamma}\right)$ is defined in terms of $D_{\gamma}, \Lambda_{\gamma}$ and $l_{\gamma}$ by

$$
S_{N}\left(n ; l_{\gamma}\right)=\left|\operatorname{det}\left(I-\left(N_{\gamma} \Lambda_{\gamma}\right)^{n}\right)\right| e^{-n \rho_{N} l_{\gamma}},
$$

where the matrix $I-\left(N_{\gamma} \Lambda_{\gamma}\right)^{n}$ has dimension $(N-1) \times(N-1)$. The integral is the contribution to the trace of the trivial class, i.e. the identity element of the group and it will soon be computed.

The geometric description. This follows if we remind the definition of the axis of a $\gamma \in \Gamma$ : it is the unique invariant geodesic in $H^{N}$ joining $x$ to $\gamma x$. When we identify $x$ with $\gamma x$ in the quotient manifold $\mathcal{M}$, the axis clearly projects to a closed geodesic in $\mathcal{M}$. A simple calculation with the Poincaré metric shows that the length of the portion of the axis between $x$ and $\gamma x$ is just $l_{\gamma}$, the logarithm of the dilatation factor $N_{\gamma}$. Thus, there is a closed geodesic of length $l_{\gamma}$ associated with such a $\gamma$. If $\gamma$ is primitive the geodesic will go once from $x$ to $\gamma x$ in $\mathcal{M}$. If $\gamma=\delta^{n}$ with $\delta$ primitive, then $l_{\gamma}=\ln N_{\delta}^{n}=n \ln N_{\delta}=n l_{\delta}$. Hence, the geodesic will go $n$ times from $x$ to $\gamma x$ and $n$ is the winding number. It is evident that the closed geodesic depends only on the conjugacy class $\{\gamma\}$. If the class is primitive, the associated geodesic is likewise called primitive. The converse is also true, since every geodesic loop in $\mathcal{M}$, say $J$, must be the projection of a geodesic in $H^{N}$ joining $x$ and $\gamma x$ for some $\gamma \in \Gamma$ and $x \in H^{N}$ (because $J$ is closed). If $\gamma^{\prime}=g \gamma g^{-1}$ then the axis of $\gamma^{\prime}$ projects again to $J$ (because $x, g x, \gamma x$ and $g \gamma x$ all belong to the same orbit). As a result, to every geodesic loop there corresponds a conjugacy class in $\Gamma$. Thus, we can think of each $\{\gamma\}$ as a closed geodesic and the double sum in Eq. (3.38) is geometrically the same as the sum over all the primitive geodesics in $\mathcal{M}$ (those passing only once through any given point) together with the sum over the winding numbers. Furthermore, the integral is the "direct path" contribution to the trace. Note that it is determined by the volume of the manifold and corresponds to the Weyl asymptotic leading term of the spectral density. We see that we can regard Eq. (3.38) as a generalization to $H^{N} / \Gamma$ of the familiar method of images on the torus. 
The path integral interpretation. This stems from the use of the trace formula to compute $\operatorname{Tr} \exp \left(t \Delta_{\Gamma}\right)$ and from its path integral representation, valid also on non simply connected spaces [115]. The result for $\hat{h}\left(n l_{\gamma}\right)$ is essentially $\exp \left(-n^{2} l_{\gamma}^{2} / 4 t\right)$. This is the exponential of the classical action for a geodesic of length $n l_{\gamma}$, the factor $S_{N}\left(n ; l_{\gamma}\right)$ resembles the Van Vleck-Morette determinant and the integral involving $\Phi_{N}$ the direct path contribution. The character $\chi(\gamma)$ also must be present in general, but the path integral may select one or more among them. This happens, for instance, in the path integral treatment of identical particles, where only completely symmetric or anti-symmetric wave functions are allowed. It would be certainly interesting to establish this connection rigorously.

Finally, let us see how the trace formula itself comes out. Let us define the invariant function $u(X, Y)=[\cosh d(X, Y)-1] / 2=\sinh ^{2}[d(X, Y) / 2]$ (see Eq. (3.21)) and let $K$ be an invariant integral operator, its kernel being given by $k(X, Y)$. The invariance of the operator is equivalent to the fulfilment of the condition $k(X, Y)=k(u(X, Y))$, i.e. the kernel is a function of the geodesic distance. Now to any function $k(u)$ on $[0, \infty)$ with compact support, we can associate the integral operator $K_{\Gamma, \chi}$ in the space $\mathcal{H}(\Gamma, \chi)$ defined by the kernel

$$
K_{\Gamma, \chi}(X, Y)=\sum_{\gamma \in \Gamma} \chi(\gamma) k(u(X, \gamma Y))
$$

(the sum if finite because $k(u)$ has compact support and $\Gamma$ is discrete). It can be proved that if $K_{\Gamma, \chi}$ is trace-class, then a function $h(r)$ exists such that

$$
\begin{aligned}
\sum_{j} h\left(r_{j}\right) & =\int_{\mathcal{F}_{N}} K_{\Gamma, \chi}(X, X) d X \\
& =\sum_{\gamma \in \Gamma} \int_{\mathcal{F}_{N}} \chi(\gamma) k(u(X, \gamma X)) d X=\sum_{\{\gamma\}} \chi(\gamma) \int_{\mathcal{F}_{\gamma}} k(u(X, \gamma X) d X .
\end{aligned}
$$

Once more, each number $r_{j}$ is the root of $r_{j}^{2}=\lambda_{j}-\rho_{N}^{2}$ in the upper half complex plane and the sum runs over the eigenvalues of $A_{\Gamma, \chi}$. We also denoted by $d X=r^{-N} d r d x_{1} \ldots d x_{N-1}$ the invariant volume element and the integral extends over a fundamental domain for $\Gamma$. Moreover, $\mathcal{F}_{\gamma}$ is a fundamental domain for $C(\gamma)$, the centralizer of $\gamma$ in $\Gamma$. In addition, the formula

$$
\int_{H^{N}} K(u(X, X)) d X=\int_{0}^{\infty} h(r) \Phi_{N}(r) d r
$$

holds for any trace-class integral operator acting on square integrable functions on $H^{N}$.

The connection $k(u) \leftrightarrow h(r)$ is known as the Selberg transform [5]. In order to introduce such a transform, we define the function $p(z)=\cosh ^{-1}(1+2 z)$ and its inverse $z(p)=(\cosh p-1) / 2$. For $N=2 n+1$, the Selberg transform can be written as

$$
\begin{aligned}
& k(u)=\frac{(-1)^{n}}{(4 \pi)^{n}} \hat{h}^{(n)}(p(u)), \\
& \hat{h}(p)=\frac{(4 \pi)^{n}}{\Gamma(n)} \int_{z(p)}^{\infty} k(x)[x-z(p)]^{n-1} d x,
\end{aligned}
$$

while if $N=2 n$

$$
\begin{aligned}
& k(u)=\frac{2(-1)^{n}}{(4 \pi)^{n}} \int_{u}^{\infty} \hat{h}^{(n)}(p(z))(z-u)^{-1 / 2} d z, \\
& \hat{h}(p)=\frac{(4 \pi)^{n}}{2 \pi^{1 / 2} \Gamma(n-1 / 2)} \int_{z(p)}^{\infty} k(x)[x-z(p)]^{n-\frac{3}{2}} d x,
\end{aligned}
$$

from which we can reconstruct $h(r)$ by the inverse Fourier transform. For a simple derivation of both the theorem and the formulae see Ref. [114]. By $\hat{h}^{(n)}(p(u))$ we indicate the $n^{\text {th }}$ derivative 
of $\hat{h}(p(u))$ with respect to the variable $u$. It should be noted that these formulae are a particular case of the theorem on the expansion of an arbitrary spherical function in zonal functions (the Fourier-Harish-Chandra transform), which is valid in more general cases than the ones considered here.

Evaluating the integrals in Eq. (3.42), using Selberg transform, one finally gets the Selberg trace formula. For the proof, we refer the reader to the literature (see for example Refs. 116, 114]. The central point is that the domain $\mathcal{F}_{\gamma}$ is very simple and this makes possible to perform the integral. Here, we explicitly compute the contribution due to the identity element of the isometry group, that is the term $\Omega\left(\mathcal{F}_{N}\right) k(0)$ in Eq. (3.42). The integral operator determined by $k(u(X, Y))$ is the operator function $\tilde{h}(\Delta)$ of the Laplace operator in $H^{N}, k(u)$ being related to $h(r)$ by Selberg transform. Thus we can expand $k(u(X, Y))$ over the eigenfunctions of the continuous spectrum, which have been computed previously in the hyperboloid model. We choose $Y=0$ to be the bottom of the hyperboloid. In this way $u(X, 0)=(\cosh \sigma-1) / 2$ and $k(u(X, 0))$ is a radial function. Thus, the expansion contains only zero angular momentum eigenfunctions and reads

$$
k(u(X, 0))=\int_{0}^{\infty} \Phi_{N}(r) h(r) f_{\lambda(r)}(X) f_{\lambda(r)}(0) d r,
$$

since the radial eigenfunctions have density $\Phi_{N}(r)$. They were defined so that $f_{\lambda}(0)=1$. Hence, setting $X=0$ we obtain the formula

$$
k(0)=\int_{0}^{\infty} d r h(r) \Phi_{N}(r)
$$

between Selberg transform pairs determining the contribution of the identity.

\subsubsection{The Selberg $Z$-function}

This section is devoted to a brief discussion of Selberg $Z$-function and its associated $\Xi$-function, with a heuristic derivation of their main properties. Let $C$ be a contour in the complex plane from $+i \infty$ to $+i \infty$ around the positive imaginary axis with anti-clockwise orientation and including all the numbers $i r_{j}$. Then for any $h(r)$ for which the Selberg formula holds, we may rewrite

$$
\begin{aligned}
\sum_{j} \int_{C} \frac{2 z h(-i z)}{z^{2}+r_{j}^{2}} d z= & \Omega\left(\mathcal{F}_{N}\right) \int_{-\infty}^{\infty} d r \Phi_{N}(r) \int_{C} \frac{z h(-i z)}{z^{2}+r^{2}} d z \\
& +\sum_{\{\gamma\}} \sum_{n=1}^{\infty} \frac{\chi^{n}(\gamma) l_{\gamma}}{S_{N}\left(n ; l_{\gamma}\right)} \int_{-\infty}^{\infty} d r \frac{e^{i n l_{\gamma} r}}{2 \pi} \int_{C} \frac{2 z h(-i z)}{z^{2}+r^{2}} d z,
\end{aligned}
$$

The integration with respect to the variable $r$, in the second term on the right hand side of the equation above, can be done without any problem. Since the function $\Phi_{N}(r)$ goes like $|r|^{N-1}$ for $|r| \rightarrow \infty$, in order to exchange and perform the integration with respect to $r$ also in the first term, we introduce a regularization function $f(r, \varepsilon)$, which is analytic for $\operatorname{Re} r \geq 0$, goes to zero faster than $|r|^{N-2}$ for $|r| \rightarrow \infty$ and goes to 1 for $\varepsilon \rightarrow 0$ (an example is $f(r, \varepsilon)=(r+i)^{-\varepsilon}$ with $\varepsilon>N-2$ ). Then we can easily make the integration in the $r$ complex plane and, at the end, take the limit $\varepsilon \rightarrow 0$. The final result reads

$$
\sum_{j} \int_{C} \frac{2 z h(-i z)}{z^{2}+r_{j}^{2}} d z=\pi \Omega\left(\mathcal{F}_{N}\right) \int_{C} \Phi_{N}(i z) h(-i z) d z+\int_{C} \Xi\left(z+\rho_{N}\right) h(-i z) d z,
$$

where $\Xi(z)$ is the Selberg $\Xi$-function, defined for $\operatorname{Re} z>2 \rho_{N}=N-1$ by

$$
\Xi(z)=\sum_{\{\gamma\}} \sum_{n=1}^{\infty} \frac{\chi^{n}(\gamma) l_{\gamma}}{S_{N}\left(n ; l_{\gamma}\right)} e^{-\left(z-\rho_{N}\right) n l_{\gamma}} .
$$


This shows that

$$
\sum_{j} \frac{2 z}{z^{2}+r_{j}^{2}}=\pi \Omega\left(\mathcal{F}_{N}\right) \Phi_{N}(i z)+\Xi\left(z+\rho_{N}\right)
$$

where $z \rightarrow \Phi_{N}(i z)$ has to be considered as a regular analytic distribution and the series converge in the space of regular analytic distributions 117.

The asymptotic behaviour $S_{N}\left(n ; l_{\gamma}\right) \simeq \exp \left(n \rho_{N} l_{\gamma}\right)$ for large $n$ and the known fact that the number of conjugacy classes with a given length $l_{\gamma}$ is asymptotically $l_{\gamma}^{-1} \exp \left(2 \rho_{N} l_{\gamma}\right)$, show that the $\Xi$-function is analytic in the half-plane $\operatorname{Re} z>N-1$. Thus, from Eq. (3.44) we deduce that $\Xi(z)$ extends to a meromorphic function with simple poles at $z_{j}=\rho_{N} \pm i r_{j}$ whose residues are $d_{j}$ (the eigenvalues multiplicity), if the eigenvalue $\lambda_{j} \neq \rho_{N}$ or $2 d_{j}$, if $\lambda_{j}=\rho_{N}$. Moreover, since $\Phi_{N}(z)$ is an even function, while the left hand side of Eq. (3.44) is an odd function of $z$, we have the functional equation

$$
\Xi\left(z+\rho_{N}\right)+\Xi\left(-z+\rho_{N}\right)=-2 \pi \Omega\left(\mathcal{F}_{N}\right) \Phi_{N}(i z) .
$$

It can be proved that this holds true in any dimensions [118]. In even dimensions there are also the additional poles of the density $\Phi_{N}(i z)$ at $z_{k}= \pm\left(\rho_{N}+k\right), k \geq 0$ with certain real residues, say $R_{k}$. Since $\Xi(z)$ is analytic for $\operatorname{Re} z>N-1$, it follows that $\Xi(z)$ in even dimensions has additional simple poles at $z_{k}=-k, k \geq 0$.

As shown by Selberg, the analytic properties of $\Xi(z)$ permit the existence of a function $Z(s)$ such that

$$
\ln Z(s)=-\sum_{\{\gamma\}} \sum_{n=1}^{\infty} \frac{\chi^{n}(\gamma)}{S_{N}\left(n ; l_{\gamma}\right) n} e^{-\left(s-\rho_{N}\right) n l_{\gamma}},
$$

then obviously $\Xi(s)=Z^{\prime}(s) / Z(s)$ and Eq. (3.45) becomes a functional equation for $Z(s)$ which reads

$$
Z\left(-s+\rho_{N}\right)=Z\left(s+\rho_{N}\right) \exp \int_{0}^{s} 2 \pi \Omega\left(\mathcal{F}_{N}\right) \Phi_{N}(i z) d z
$$

where the complex integral in the exponent is over any contour not crossing the poles of $\Phi_{N}(i z)$. It can be shown that the residues of the function $2 \pi \Omega\left(\mathcal{F}_{N}\right) \Phi_{N}(i z)$ are multiples of the Euler number of the manifold, so the integral is well defined. The poles of $\Xi(s)$ become poles or zeroes of $Z(s)$. More precisely, if $N$ is odd then $Z(s)$ is an entire function of order $N$ and all the poles of $\Xi(s)$ are zeroes of $Z(s)$. If $N$ is even there are the additional poles of the density $\Phi_{N}(i s)$. When the residues $R_{k}>0, Z(s)$ has poles at all negative integers. This occur precisely when $N=4 n$, as can be seen with some calculations. In all the other cases, the negative integers are zeroes of $Z(s)$ [118]. The point $s=0$ is special. In odd dimensions it is always a zero, if $\chi(\gamma)=1$. In even dimensions it can also be a non simple pole, depending on the Euler characteristic of the manifold [118].

\subsubsection{The Selberg trace formula for compact $H^{3} / \Gamma$}

From a physical point of view, this is certainly one of the most important cases. One peculiarity is the explicit realization of the isometry group as $\operatorname{PSL}(2, \mathbb{C})=S L(2, \mathbb{C}) /\{-1,1\}$ and the possibility to make use of complex numbers formalism. A second fact is the possibility to include elliptic elements into the trace formula. When $\Gamma$ is a discrete subgroup of $P S L(2, \mathbb{C})$ containing elliptic elements, the space $H^{3} / \Gamma$ is called the associated 3-orbifold. It is known that $H^{3} / \Gamma$ is always a manifold, but the Riemannian metric is singular along the axis of rotation of elliptic elements. 
Using complex numbers, we can represent $H^{3} \equiv\left\{X=(z, r) \mid z=x^{1}+i x^{2} \in \mathbb{C}, r=x^{3} \in\right.$ $(0, \infty)\}$, with the Riemannian metric $d l^{2}=\left(d \bar{z} d z+d r^{2}\right) / r^{2}$. The group $S L(2, \mathscr{C})$ acts on $H^{3}$ in the following way. Given a matrix $\sigma \in S L(2, \mathscr{C})$

$$
\sigma=\left(\begin{array}{ll}
a & b \\
c & d
\end{array}\right)
$$

and $X \in H^{3}$, one defines the following orientation preserving isometry [119, 120]:

$$
\sigma X=\left(\frac{(a z+b)(\bar{c} \bar{z}+\bar{d})+a \bar{c} r^{2}}{|c z+d|^{2}+|c|^{2} r^{2}}, \frac{r}{|c z+d|^{2}+|c|^{2} r^{2}}\right)
$$

It is evident that the isometry determines $\sigma$ up to sign. Eq. (3.47) is simply a special way to rewrite the generators of $G_{3}$ (see Sec. 3.4).

As in the general case, all elements of $P S L(2, \mathbb{C})$ belong to one of the following conjugacy classes: elliptic, loxodromic and parabolic and, in this case, we also discuss the case corresponding to $\Gamma$ containing elliptic elements. From the normal form of the isometries we have discussed in Sec.(3.4), we see that every loxodromic element $\gamma \in \Gamma$ is conjugate in $P S L(2, \mathbb{C})$ to a unique element $D(\gamma)$ of the form

$$
D(\gamma)=\left(\begin{array}{cc}
a_{\gamma} & 0 \\
0 & a_{\gamma}^{-1}
\end{array}\right), \quad \quad\left|a_{\gamma}\right|>1 .
$$

From Eq. (3.47) we see that $D(\gamma)$ is a dilatation in the vertical line $H^{1}$ with dilatation factor $\left|a_{\gamma}\right|^{2}$. The number $N_{\gamma}=\left|a_{\gamma}\right|^{2}$ is called the norm of $\gamma$ and $l_{\gamma}=\ln N_{\gamma}$. The phase of $a_{\gamma}$ is an $S O(2)$ rotation in $\mathbb{C}$ around $H^{1}$. Every elliptic element $\alpha \in \Gamma$ is conjugate in $P S L(2, \mathbb{C})$ to a unique element $D(\alpha)$ of the form

$$
D(\alpha)=\left(\begin{array}{cc}
\xi_{\alpha} & 0 \\
0 & \xi_{\alpha}^{-1}
\end{array}\right), \quad\left|\xi_{\alpha}\right|=1
$$

From Eq. (3.47), we see that $D(\alpha)$ is a rotation in $\mathbb{C}$ around the origin with angle $\theta=2 \arg \xi_{\alpha}$. Let $C_{\gamma}$ be the centralizer of $\gamma$ in $\Gamma$. Again $C_{\gamma}$ in infinite cyclic but this time it may contain a discrete group of rotations around the axis of $\gamma$ (since these commute with $\gamma$ ). We denote by $R_{\gamma}$ the one with minimal rotation angle and by $m_{\gamma}$ its order, so that $\left(R_{\gamma}\right)^{m_{\gamma}}=1$. Similarly, the centralizer $C_{\alpha}$ of any elliptic element is infinite cyclic so it must contain loxodromic elements [119, 121]. The reason is very simple. From the normal form of $\alpha$ and $\gamma$ given by Eqs. (3.48) and (3.49), it follows that the dilatations in $H^{1}$ commute with $D(\alpha)$. Hence, $C_{\alpha}$ will contain a discrete group of dilatations on $H^{1}$ and it is a known fact that such a group is discontinuous if and only if it is infinitely cyclic. Let us choose a primitive element among the loxodromic elements in $C_{\alpha}$ and call $N_{\alpha}$ its norm. This is uniquely determined by $\alpha$, since the square modulus eliminates the rotational part. Moreover, each elliptic $\alpha$ is the power of a primitive elliptic element whose order is denoted by $m_{\alpha}$ (simply take the rotation with minimal rotation angle around the axis of $\alpha$ ). Finally, let we choose a character $\chi$ for $\Gamma$.

From Eq. (3.39) we have $2 \pi^{2} \Phi_{3}(r)=r^{2}$ and $\rho_{3}=1$. Moreover Eq. (3.41) gives

$$
S_{3}\left(n ; l_{\gamma}\right)=\left|a_{\gamma}^{n}-a_{\gamma}^{-n}\right|^{2},
$$

where the determinant has been written using the $S L(2, \mathcal{C})$ representation. Nevertheless, the contribution of primitive loxodromic elements to the trace is not yet determined if there are elliptic conjugacy classes. This is because in the strictly loxodromic case all the conjugacy classes have been parametrized by the powers $\gamma^{n}$, with $\gamma$ primitive. In the present case, they are parametrized by the powers $\gamma^{n}\left(R_{\gamma}\right)^{j}$, where $0 \leq j<m_{\gamma}$ and $R_{\gamma}$ is the rotation of minimal angle 
around the axis of $\gamma$ whose order was denoted by $m_{\gamma}$. In fact, two different elements among these powers are not conjugate in $\Gamma$ and it is not hard to see that they form a complete representative system of all conjugacy classes, if $\gamma$ runs through all primitive loxodromic elements.

The Selberg trace formula for scalar fields, in the presence of elliptic elements is now [119]

$$
\begin{aligned}
\sum_{j} h\left(r_{j}\right)=\frac{\Omega\left(\mathcal{F}_{3}\right)}{2 \pi^{2}} \int_{0}^{\infty} & h(r) r^{2} d r \\
& +\sum_{\{\alpha\}} \sum_{n=0}^{m_{\alpha}-1} \frac{\chi^{n}(\alpha) \ln N_{\alpha}}{m_{\alpha}\left|\left(\xi_{\alpha}\right)^{n}-\left(\xi_{\alpha}\right)^{-n}\right|^{2}} \hat{h}(0) \\
& +\sum_{\{\gamma\}} \sum_{n=1}^{\infty} \sum_{j=0}^{m_{\gamma}-1} \frac{\chi^{n}(\gamma) l_{\gamma}}{m_{\gamma}\left|\left(a_{\gamma}\right)^{n} \zeta^{j}-\left(a_{\gamma}\right)^{-n} \zeta^{-j}\right|^{2}} \hat{h}\left(n l_{\gamma}\right) .
\end{aligned}
$$

The summations are extended over the primitives elliptic $\{\alpha\}$ and hyperbolic $\{\gamma\}$ conjugacy classes in $\Gamma$, as we have done in arbitrary dimensions. All symbols have been defined above but the complex numbers $\zeta, \zeta^{-1}$. They are the eigenvalues of $R_{\gamma}$ and have modulus one. Because $R_{\gamma}$ has finite order $m_{\gamma}, \zeta^{2 m_{\gamma}}=1$ so $\zeta$ is a primitive $2 m_{\gamma}$-th root of unity. Once more, the integral is the contribution of the identity class with the density $\Phi_{3}(r)$.

The general discussion we have presented in Sec.(3.4.1) about the trace formula remains valid in this case, except for the geometric description. In particular, Eq. (3.42) is the starting point for the trace formula also if elliptic elements are allowed.

For use later on, it is convenient to introduce the compact notation

$$
\begin{aligned}
& E=\sum_{\{\alpha\}} \sum_{n=0}^{m_{\alpha}-1} \frac{\chi^{n}(\alpha) \ln N_{\alpha}}{m_{\alpha}\left|\xi_{\alpha}^{n}-\xi_{\alpha}^{-n}\right|^{2}}, \\
& H(\gamma ; n)=\sum_{j=0}^{m_{\gamma}-1} \frac{\chi^{n}(\gamma) l_{\gamma}}{m_{\gamma}\left|a(\gamma)^{n} \zeta^{j}-a^{-n}(\gamma) \zeta^{-j}\right|^{2}} .
\end{aligned}
$$

The real number $E$ is called the elliptic number of the manifold $H^{3} / \Gamma$. Its meaning in terms of the geometric properties of $H^{3} / \Gamma$ is unclear. However, if any two associated 3-orbifolds have the same eigenvalue spectrum, then the volumes and the elliptic numbers are the same. This is known as (part) of Huber theorem.

The Selberg $\Xi$-function is here defined as in $N$-dimensions by

$$
\Xi(z)=\sum_{\{\gamma\}} \sum_{n=1}^{\infty} \sum_{j=0}^{m_{\gamma}-1} \frac{\chi^{n}(\gamma) l_{\gamma}}{m_{\gamma}\left|\left(a_{\gamma}\right)^{n} \zeta^{j}-\left(a_{\gamma}\right)^{-n} \zeta^{-j}\right|^{2}} e^{-(z-1) n l_{\gamma}}
$$

and again, with indefinite integration $Z(s)=\exp \int \Xi(z) d z$. This function is an entire function of order three [119] and satisfies the functional equation

$$
Z(-s+1)=Z(s+1) \exp \left(-\frac{\Omega\left(\mathcal{F}_{3}\right) s^{3}}{3 \pi}+2 E s\right) .
$$

It may be of interest to mention that if $\lambda=1$ is an eigenvalue of multiplicity $m$, then $s=1$ is a zero of $Z(s)$ of multiplicity $2 m$. 


\subsubsection{The Selberg trace formula for compact $H^{2} / \Gamma$}

Here again the use of complex numbers is useful. We take $H^{2}=\{z \in \mathbb{C} \mid \operatorname{Im} z>0\}$. We shall be interested in the so called Fuchsian group of the first kind, namely a discrete group of motion of $H^{2}$ for which there exists a fundamental domain with a finite invariant measure. The group of all orientation preserving isometries is $\operatorname{PSL}(2, \mathbb{R})=S L(2, \mathbb{R}) /\{1,-1\})$, acting on $H^{2}$ by fractional linear transformations $z \rightarrow(a z+b) /(c z+d)$. According to a classical result due to Fricke, any Fuchsian group of the first kind is finitely generated and a certain standard system of generators and relations may be given (see for example Ref. [122]). The measure of the fundamental domain can be computed in terms of signature $\left(g, m_{1}, \ldots, m_{l}, h\right)$

$$
\Omega\left(\mathcal{F}_{2}\right)=2 \pi\left[2 g-2+\sum_{j=1}^{l}\left(1-\frac{1}{m_{j}}\right)\right],
$$

where $g$ is the genus and the numbers $m_{j}$ and $h$ are associated with elliptic and parabolic generators respectively. If we allow $\Gamma$ to contain elliptic, but no parabolic elements, the orbifold $H^{2} / \Gamma$ will be compact, but the Riemannian metric will be singular at the fixed points (not lines as in $H^{3} / \Gamma$ ) of the elliptic elements. The canonical form of a loxodromic element is easily seen to be a dilatation

$$
\gamma=\left(\begin{array}{cc}
a & 0 \\
0 & a^{-1}
\end{array}\right), \quad \quad a>1 .
$$

The number $N_{\gamma}=a^{2}$ is the dilatation factor of $\gamma$ or the norm of $\gamma$. Since the matrix trace is invariant under conjugation, we see that $\gamma$ is loxodromic if and only if $(\operatorname{Tr} \gamma)^{2}>4$. Every hyperbolic element $\gamma$ is always a power of a primitive one, which is uniquely determined by $\gamma$ itself. This is the dilatation with the smallest possible dilatation factor.

The canonical form of an elliptic element is

$$
\alpha=\left(\begin{array}{cc}
\cos \theta & -\sin \theta \\
\sin \theta & \cos \theta
\end{array}\right) .
$$

This is a non linear rotation around the point $i=(0,1)$. We call $\theta \in[0, \pi]$ the angle of rotation. Thus $\alpha$ is elliptic if and only if $(\operatorname{tr} \alpha)^{2}<4$. This non linear realization of rotations is peculiar of $H^{2}$, since there are no true linear rotations around the vertical line $H^{1}$. Every elliptic element $\alpha$ is also a power of a uniquely determined primitive one. This is also the non linear rotation around the fixed point of $\alpha$ with the smallest possible angle. The angle is $\theta_{0}=\pi / \mathrm{m}$ for some integer $m$ (otherwise the group is not discrete). The order of the corresponding rotation is thus $m$ (actually, the $m$-th power of this primitive rotation is -1 , but this is the identity in $\operatorname{PSL}(2, \mathbb{R}))$. Finally, an element $\beta$ is parabolic if and only if $(\operatorname{tr} \beta)^{2}=4$.

Eq. (3.39) gives $2 \pi \Phi_{2}(r)=r \tanh \pi r$ and $\rho_{2}=1 / 2$. Furthermore, in 2-dimensions the rotation $\Lambda_{\gamma}=1$ for all $\gamma$. Then Eq. (3.41) simply gives

$$
S_{2}\left(n ; l_{\gamma}\right)=2 \sinh \left(\frac{n l_{\gamma}}{2}\right) \text {. }
$$

The contribution of hyperbolic elements is thus determined as in $N$-dimensions. But now we have also elliptic elements. The Selberg trace formula for scalar fields on compact $H^{2} / \Gamma$ including elliptic conjugacy classes is 116, 123

$$
\sum_{j} h\left(r_{j}\right)=\frac{\Omega\left(\mathcal{F}_{2}\right)}{2 \pi} \int_{0}^{\infty} d r h(r) r \tanh \pi r
$$




$$
\begin{aligned}
& +\sum_{\{\gamma\}} \sum_{n=1}^{\infty} \frac{\chi^{n}(\gamma) l_{\gamma}}{2 \sinh \left(\frac{n l_{\gamma}}{2}\right)} \hat{h}\left(n l_{\gamma}\right) \\
& +\sum_{\{\alpha\}} \sum_{j=1}^{m_{\alpha}-1} \frac{\chi^{j}(\alpha)}{2 m_{\alpha} \sin \frac{j \pi}{m_{\alpha}}} \int_{-\infty}^{\infty} \frac{\exp \left(-\frac{2 \pi r j}{m_{\alpha}}\right)}{1+\exp (-2 \pi r)} h(r) d r .
\end{aligned}
$$

The summations are extended over the primitive elliptic $\{\alpha\}$ and hyperbolic $\{\gamma\}$ conjugacy classes in $\Gamma$ and each number $m_{\alpha}$ is the order of the primitive representative $\alpha$.

In 2-dimensions and without elliptic elements, the Selberg $Z$-function admits a simple product representation

$$
Z(s)=\prod_{\{\gamma\}} \prod_{k=0}^{\infty}\left(1-\chi(\gamma) e^{-(s+k) l_{\gamma}}\right)
$$

and $\Xi(s)=Z^{\prime}(s) / Z(s)$. Such infinite product representations also exist in any dimension but they are much more complicated. $Z(s)$ is an entire function of order two, with trivial zeroes at $s_{k}=-k, k=1,2, . ., \infty$, of multiplicity $(2 g-2)(2 k+1)$, where $g$ is the genus of the Riemann surface $H^{2} / \Gamma$. For trivial character, $s=0$ is a zero of multiplicity $2 g-1$ and $s=1$ is a simple zero. Moreover, there are non trivial zeroes at $s_{j}=1 / 2 \pm i r_{j}$, in agreement with the general discussion. The functional equation is

$$
Z(1 / 2-s)=Z(1 / 2+s) \exp \left[-\Omega\left(\mathcal{F}_{2}\right) \int_{0}^{s} z \tan (\pi z) d z\right]
$$

Since $Z(s)$ has a simple zero at $s=1$ for trivial character, it follows that $Z^{\prime}(1)$ is non zero. This quantity is important because it determines the functional determinant of the Laplace operator on a Riemann surface.

\subsubsection{Recurrence relations for heat kernel and $\zeta$-function}

We know that for physical applications heat kernel and $\zeta$-function related to the LaplaceBeltrami operator $\Delta_{N}$ acting on fields in $H^{N} / \Gamma$ are relevant. Then we choose $h(r)=\exp \left(-t\left(r^{2}+\right.\right.$ $\left.\varrho_{N}^{2}\right)$ ), compute the trace of the heat kernel using Eq. (3.38) and then, using Eq. (2.13) we shall also get the $\zeta$-function. Alternatively, we can obtain the $\zeta$-function by choosing $h(r)=$ $\left(r^{2}+\varrho_{N}^{2}\right)^{-s}$ and directly apply the Selberg trace formula. It is convenient to distinguish between the contribution to these quantities coming from the identity and the hyperbolic elements of the isometry group. In 2 and 3-dimensions also the contribution of elliptic elements shall be taken into account.

By a simple calculation we obtain

$$
\begin{aligned}
\operatorname{Tr} e^{-t \mathrm{E}_{N}}= & \Omega\left(\mathcal{F}_{N}\right) e^{-t \alpha^{2}} \int_{0}^{\infty} e^{-t r^{2}} \Phi_{N}(r) d r+\frac{e^{-t \alpha^{2}}}{(4 \pi t)^{1 / 2}} \sum_{\{\gamma\}} \sum_{n=1}^{\infty} \frac{\chi^{n}(\gamma) l_{\gamma}}{S_{N}\left(n ; l_{\gamma}\right)} e^{-\left(n l_{\gamma}\right)^{2} / 4 t}, \\
= & K_{I}\left(t \mid \mathrm{E}_{N}\right)+K_{H}\left(t \mid \mathrm{E}_{N}\right) \\
\zeta\left(s \mid \mathrm{E}_{N}\right)= & \Omega\left(\mathcal{F}_{N}\right) \int_{0}^{\infty}\left(r^{2}+\alpha^{2}\right)^{-s} \Phi_{N}(r) d r \\
& \quad+\frac{1}{\sqrt{\pi} \Gamma(s)} \sum_{\{\gamma\}} \sum_{n=1}^{\infty} \frac{\chi^{n}(\gamma) l_{\gamma}}{S_{N}\left(n ; l_{\gamma}\right)}\left(\frac{2 \alpha}{n l_{\gamma}}\right)^{\frac{1}{2}-s} K_{\frac{1}{2}-s}\left(n l_{\gamma} \alpha\right), \\
= & \zeta_{I}\left(s \mid \mathrm{E}_{N}\right)+\zeta_{H}\left(s \mid \mathrm{E}_{N}\right) .
\end{aligned}
$$

For the sake of generality and for future applications, we again consider the operator $\mathrm{E}_{N}=$ $-\Delta_{N}+\alpha^{2}+\kappa \varrho_{N}^{2}$. Note that in contrast with the spherical case now $\alpha^{2}$ is always positive, since 
$\kappa<0 . \quad \alpha^{2}=0$ and $\alpha^{2}=m^{2}-\kappa \varrho_{N}^{2}$ give the conformal and minimal couplings respectively, while $\alpha^{2}=-\kappa \varrho_{N}^{2}$ corresponds to the massless case (note that we think of $H^{N}$ as the spatial section of an ultrastatic $N+1$ dimensional manifold. Only in this case $\alpha^{2}=0$ corresponds to the conformal coupling).

Using recurrence relation (3.40) we easily get a recurrence formula for $K_{I}\left(t \mid \mathrm{E}_{N}\right)$ which is very similar to the corresponding equation on $S^{N}$, Eq. (3.14). It reads

$$
\frac{K_{I}\left(t \mid \mathrm{E}_{N+2}\right)}{\Omega\left(\mathcal{F}_{N+2}\right)}=-\frac{1}{2 \pi N \Omega\left(\mathcal{F}_{N}\right)}\left[\partial_{t}+\alpha^{2}+\kappa \varrho_{N}^{2}\right] K_{I}\left(t \mid \mathrm{E}_{N}\right) .
$$

We also have recurrence formulae for the spectral coefficients and also for $\zeta_{I}\left(s \mid \mathrm{E}_{N}\right)$, that is

$$
\begin{aligned}
& \frac{K_{n}\left(\mathrm{七}_{N+2}\right)}{\Omega\left(\mathcal{F}_{N+2}\right)}=-\frac{1}{2 \pi N \Omega\left(\mathcal{F}_{N}\right)}\left[\frac{n+N}{2} K_{n}\left(\mathrm{E}_{N}\right)+\left(\alpha^{2}+\kappa \varrho_{N}^{2}\right) K_{n-2}\left(\mathrm{E}_{N}\right)\right], \\
& \frac{\zeta_{I}\left(s \mid \mathrm{E}_{N+2}\right)}{\Omega\left(\mathcal{F}_{N+2}\right)}=-\frac{1}{2 \pi N \Omega\left(\mathcal{F}_{N}\right)}\left[\left(\alpha^{2}+\kappa \varrho_{N}^{2}\right) \tilde{\zeta}_{I}\left(s \mid \mathrm{E}_{N}\right)-\tilde{\zeta}_{I}\left(s-1 \mid \mathrm{E}_{N}\right)\right] .
\end{aligned}
$$

Knowing the partition function on $H^{2}$ and $H^{3}$ and using this latter equation we can obtain the partition function for a scalar field on $H^{N}$. It is easy to see that Eq. (3.52) is valid also for the corresponding regularized functions according to Eq. (2.34). Equations above are formally the same ones that we had in Sec. 3.2 for the spherical case. The only difference is the sign of the curvature. The specific computations for $H^{2} / \Gamma$ and $H^{3} / \Gamma$ are collected in Appendix $\mathrm{G}$.

The topological contribution to $\zeta$ due to hyperbolic elements (the last term in Eq. (3.51)) can be conveniently expressed in terms of the logarithmic derivative of Selberg $Z$-function. In fact, using a suitable integral representation for $K_{\nu}$ (see Appendix D) and definition (3.43) we have

$$
\zeta_{H}\left(s \mid \mathrm{E}_{N}\right)=\frac{\alpha^{1-2 s} \sin \pi s}{\pi} \int_{1}^{\infty}\left(u^{2}-1\right)^{-s} \Xi\left(u \alpha+\varrho_{N}\right) d u,
$$

which gives an integral representation of $\zeta_{H}\left(s \mid \mathrm{E}_{N}\right)$ valid for $\operatorname{Re} s<1$. Deriving the latter expression at the point $s=0$, we obtain the expression, which is relevant in the discussion of the factorization formulae of the next subsection

$$
\zeta_{H}^{\prime}\left(0 \mid \mathrm{E}_{N}\right)=-\ln Z\left(\alpha+\varrho_{N}\right) .
$$

In the particular but important cases in which the hyperbolic section has 2 or 3 dimensions, we are able to compute also the elliptic contributions to the $\zeta$-function. The results are reported in Appendix C, where for reader convenience we collect some representations for $\zeta$-function on $H^{3} / \Gamma$ and $H^{2} / \Gamma$.

\subsubsection{A factorization formula and zero modes}

We finish the section with some considerations on the possible presence of zero modes. First of all we define the numbers $b_{k}^{N}$ by means of

$$
\begin{array}{rlrl}
\Phi_{N}(r) & =\sum_{k=0}^{(N-3) / 2} b_{k}^{N} r^{2 k} \frac{r^{2}}{2 \pi^{2}}, & \text { for odd } N \geq 3, \\
\Phi_{N}(r)=\sum_{k=0}^{(N-2) / 2} b_{k}^{N} r^{2 k} \frac{r \tanh \pi r}{2 \pi}, & \text { for even } N \geq 2 .
\end{array}
$$


These can be easily computed making use of Eq. (3.40). For example we have

$$
\begin{array}{ll}
a_{0}^{3}=1, & a_{0}^{5}=a_{1}^{5}=\frac{1}{6 \pi}, \\
a_{0}^{2}=1, & a_{0}^{4}=\frac{1}{16 \pi},
\end{array}
$$

In this way we can write

$$
\begin{aligned}
& \zeta_{I}\left(s \mid \mathrm{E}_{N}\right)=\Omega\left(\mathcal{F}_{N}\right) \sum_{k=0}^{(N-3) / 2} b_{k}^{N} \frac{\Gamma\left(k+\frac{3}{2}\right) \Gamma\left(s-k-\frac{3}{2}\right)}{4 \pi^{2} \Gamma(s)} \alpha^{3+2 k-2 s}, \\
& \zeta_{I}\left(s \mid \mathrm{E}_{N}\right)=\Omega\left(\mathcal{F}_{N}\right) \sum_{k=0}^{(N-2) / 2} b_{k}^{N}\left[\frac{\Gamma(k+1) \Gamma(s-k-1)}{4 \pi \Gamma(s)} \alpha^{2+2 k-2 s}\right. \\
&\left.-\frac{1}{\pi} \int_{0}^{\infty} \frac{\left(r^{2}+\alpha^{2}\right)^{-s} r^{2 k+1}}{1+e^{2 \pi r}} d r\right],
\end{aligned}
$$

which are valid for odd $N \geq 3$ and even $N \geq 2$ respectively. By setting $\zeta_{I}\left(s \mid \mathrm{E}_{N}\right)=\frac{K_{N}\left(\mathrm{E}_{N}\right)}{\Gamma(s+1)}+$ $\frac{I\left(s \mid \mathrm{Ł}_{N}\right)}{\Gamma(s)}$, for odd $N \geq 3$ we easily obtain

$$
K_{N}\left(\mathrm{E}_{N}\right)=0, \quad I\left(0 \mid \mathrm{E}_{N}\right)=\Omega\left(\mathcal{F}_{N}\right) \sum_{k=0}^{(N-3) / 2} b_{k}^{N} \frac{\Gamma\left(k+\frac{3}{2}\right) \Gamma\left(-k-\frac{3}{2}\right)}{4 \pi^{2}} \alpha^{3+2 k},
$$

while for even $N \geq 2$, after some calculations we get

$$
\begin{array}{r}
K_{N}\left(\mathrm{E}_{N}\right)=\Omega\left(\mathcal{F}_{N}\right) \sum_{k=0}^{(N-2) / 2} b_{k}^{N}\left[\frac{\left(-\alpha^{2}\right)^{k+1}}{4 \pi(k+1)}-\frac{\Gamma(2 k+2)\left(1-2^{-2 k-1}\right) \zeta_{R}(2 k+2)}{\pi(2 \pi)^{2 k+2}}\right], \\
I\left(0 \mid \mathrm{E}_{N}\right)=-\gamma K_{N}\left(\mathrm{E}_{N}\right)+\Omega\left(\mathcal{F}_{N}\right) \sum_{k=0}^{(N-2) / 2} b_{k}^{N}\left[\frac{\left(-\alpha^{2}\right)^{k+1}}{4 \pi(k+1)}\left(C_{k+1}-\ln \alpha^{2}\right)\right. \\
\left.+\frac{1}{\pi} \int_{0}^{\infty} \frac{\ln \left(r^{2}+\alpha^{2}\right) r^{2 k+1}}{1+e^{2 \pi r}} d r\right],
\end{array}
$$

where $\gamma$ is the Euler-Mascheroni constant and $C_{n}=\sum_{k=1}^{n} 1 / k$.

Now we consider the pure Laplacian $L=-\Delta_{N}$ in $H^{N} / \Gamma$ and take into consideration the possible presence of $\mathcal{N}$ zero-modes. In order to deal with them, we have already pointed out in Sec. 2.2 that it is convenient to make use of the operator $L_{\lambda}=L+\lambda$, with $\lambda$ a suitable non negative parameter and define the determinant of $L$ by means of Eq. (2.23), namely

$$
\operatorname{det} L=\frac{e^{-\gamma K_{N}(L)}}{\mathcal{N} !} \frac{d^{\mathcal{N}}}{d \lambda^{\mathcal{N}}} \exp \left[-G\left(0 \mid L_{\lambda}\right)\right]
$$

Recalling the definition of $G(s \mid L)=\Gamma(s) \zeta(s \mid L)-K_{N}(L) / s$ (Eq. (2.20)), Eqs. (3.51) and (3.54) we can write

$$
G\left(0 \mid L_{\lambda}\right)=I\left(0 \mid L_{\lambda}\right)-\ln Z\left(\rho_{N}+\sqrt{\rho_{N}^{2}+\lambda}\right) .
$$

Looking at Eqs. (3.55) and (3.56), we see that $I\left(0 \mid \mathrm{E}_{N}\right)$ is well defined also in the limit $\alpha^{2} \rightarrow \varrho_{N}^{2}$, which corresponds to the pure Laplacian. This means that possible singularities for $\lambda \rightarrow 0$ in the determinant of the Laplacian come from Selberg $Z$-function and this is in 
agreement with the fact that zero-modes come from non trivial topology. In fact we have the factorization formula

$$
Z\left(\rho_{N}+\sqrt{\rho_{N}^{2}+\lambda}\right)=\lambda^{\mathcal{N}} \exp \left[\gamma K_{N}\left(L_{\lambda}\right)+I\left(0 \mid L_{\lambda}\right)-f^{\prime}\left(0 \mid L_{\lambda}\right)\right]
$$

where $f\left(s \mid L_{\lambda}\right)=\zeta\left(s \mid L_{\lambda}\right)-\mathcal{N} \lambda^{-s}$ is well defined in the limit $\lambda \rightarrow 0$. Eq. (3.59) tells us that if $\mathcal{N}$ zero-modes are present, the Selberg $Z(s)$-function has a zero of multiplicity $\mathcal{N}$ at $s=2 \rho_{N}=$ $N-1$, in agreement with the general results reported in Ref. [118] and discussed in Sec. 3.4.2. However, the presence of zero modes depends on the characters $\chi$.

Finally, by Eq. (3.57) we get the regularized determinant for the Laplace operator in the form

$$
\operatorname{det} L=\frac{1}{\mathcal{N} !} \exp \left[-\gamma K_{N}(L)-I(0 \mid L)\right] Z^{(\mathcal{N})}\left(2 \rho_{N}\right) .
$$




\section{Zero temperature quantum properties on ultrastatic mani- folds with constant curvature section}

Here we recall some basic properties concerning quantum field theory in ultrastatic space-times. It has been proved in Ref. 124 that any conformally flat, ultrastatic space-time is locally the Minkowski space, the Einstein static universe or the open Einstein universe, namely $\mathbb{R} \times H^{3}$ with the product metric. Actually this holds true in arbitrary dimensions, in which case we denote by $\mathcal{M}^{\mathcal{N}}$ the corresponding spatial section. The global topology of such spaces however can be very involved and firstly only simply connected space-times have been considered. Later, in Refs. 125, 126, 127] field theories on space-times carrying non-trivial topology have been considered. When $\mathcal{M}^{\mathcal{N}}$ has constant curvature, the spaces $\mathbb{R} \times \mathcal{M}^{\mathcal{N}}$ are all conformally flat. The question arises whether they are globally hyperbolic, due to the importance that this property has for the quantum theory. One can easily see that this cannot be the case if the spatial section is incomplete as a Riemannian manifold. It is a known fact that any ultrastatic space-time is globally hyperbolic provided the spatial section is a complete Riemannian manifold [128]. Furthermore, in the physical 4-dimensional case, we have the Fulling-Narcowich-Wald theorem [129], stating that the Hadamard elementary solution of the wave equation has the singularity structure of the Hadamard form. The consequences of this for the renormalization program are well known. When the spatial section of space-time is simply connected and maximally symmetric, it can be shown that the renormalized vacuum stress tensor takes the general form

$$
T_{i j}=\frac{1}{3} \rho_{v}\left[g_{i j}+4 \xi_{i} \xi_{j}\right]
$$

and thus it is completely determined by the vacuum energy density. Here $\xi_{i}$ is the global timelike killing field which defines the given ultrastatic space-time. Now we discuss these issues for the three possible ultrastatic geometries but admitting non trivial global topology.

\subsection{The vacuum energy for massive scalar fields on $\mathbb{R} \times T^{N}$}

This is a well studied case, see for example Ref. [130]. The regularized vacuum energy for a scalar field on an ultrastatic manifold can be obtained using Eq. (2.72). Here we have to deal with an operator of the kind $L_{D}=-\partial_{\tau}^{2}+\mathrm{E}_{N}$ with $D=N+1$ and $\mathrm{E}_{N}=-\Delta_{N}+m^{2}$ represents an operator acting on fields in $T^{N}$. As in Sec. 3.2, we call $r_{i}(i=1, \ldots, N)$ the radii of the circles. Then, using Eqs. (2.34), (2.72) and (3.4) for the vacuum energy $E_{v}$ we obtain, for even $D$

$$
\begin{aligned}
E_{v}=\nu\left(\frac{-1}{4 \pi}\right)^{\frac{D}{2}} \frac{\Omega_{N} m^{D}}{(D / 2) !} & {\left[\ln \left(m^{2} \ell^{2}\right)-C_{D / 2}\right] } \\
& -\frac{\nu \Omega_{N} m^{D}}{(4 \pi)^{\frac{N}{2}} \Gamma\left(\frac{D+1}{2}\right)} \sum_{\vec{n} \neq 0} \int_{1}^{\infty}\left(u^{2}-1\right)^{\frac{N}{2}} e^{-2 \pi m|\vec{n} \cdot \vec{r}| u} d u,
\end{aligned}
$$

while for odd $D$

$$
E_{v}=-\frac{\nu \Omega_{N} \Gamma(-D / 2) m^{D}}{(4 \pi)^{\frac{D}{2}}}-\frac{\nu \Omega_{N} m^{D}}{(4 \pi)^{\frac{N}{2}} \Gamma\left(\frac{D+1}{2}\right)} \sum_{\vec{n} \neq 0} \int_{1}^{\infty}\left(u^{2}-1\right)^{\frac{N}{2}} e^{-2 \pi m|\vec{n} \cdot \vec{r}| u} d u,
$$

with $C_{D}=\sum_{k=1}^{D} 1 / k$ and $\vec{n} \in \mathbb{Z}^{N}$.

Now we specialize this result to a 3-torus universe and describe the stress tensor for the untwisted, massive scalar field. With the three radii of the torus let us form the four vector $N^{i}=2 \pi\left(0, r_{1}, r_{2}, r_{3}\right)$, the integer vector $\vec{n}=\left(n_{1}, n_{2}, n_{3}\right)$ and let $\xi^{i}$ be the globally time-like Killing field of $\mathbb{R} \times T^{3}$. In the natural coordinate system $(t, \vec{x}), \vec{x} \in T^{3}$ it takes the form 
$\xi^{i}=(1,0,0,0)$. Moreover we denote $\varrho^{i}=(x-y)^{i}, x, y \in \mathbb{R} \times T^{3}$. The Hadamard elementary function on the torus is then given as the images sum

$$
G_{T}^{(1)}(x, y)=:<0|\{\phi(x), \phi(y)\}| 0>=\sum_{\vec{n}} G^{(1)}\left(g_{i j}\left(\varrho^{i}+N^{i}\right)\left(\varrho^{j}+N^{j}\right)\right) .
$$

Here, $\{\phi(x), \phi(y)\}$ is the anticommutator and $G^{(1)}(x, y)$ is the Hadamard function for flat Minkowski space (see for example Ref. [3] and references therein). This function then defines a global vacuum state for the torus universe. For a minimally coupled scalar field, the quantum expectation value of the stress tensor in this vacuum is defined by

$$
T_{i j}=\frac{1}{2} \lim _{y \rightarrow x}\left[\nabla_{x^{i}} \nabla_{y^{j}}-\frac{1}{2} g_{i j}\left(\nabla_{x^{k}} \nabla^{y^{k}}+m^{2}\right)\right] G_{s}^{(1)}(x, y),
$$

where the Minkowski $(\vec{n}=0)$ term has been subtracted from $G_{T}^{(1)}(x, y)$ in order to define the singularity free function $G_{s}^{(1)}(x, y)$. As a result, the stress tensor takes the form

$$
T_{i j}=A g_{i j}+B g_{i \mu} g_{j \nu} N^{\mu} N^{\nu},
$$

in which

$$
\begin{aligned}
& A=-\frac{m^{4}}{4 \pi^{2}} \sum_{n \neq 0} \frac{1}{U_{n}^{2}} K_{2}\left(U_{n}\right), \\
& B=-\frac{m^{6}}{4 \pi^{2}} \sum_{n \neq 0}\left[\frac{3}{U_{n}^{4}} K_{2}\left(U_{n}\right)+\frac{1}{U_{n}^{3}} K_{1}^{\prime \prime}\left(U_{n}\right)\right],
\end{aligned}
$$

where $U_{n}=m \sqrt{g_{i j} N^{i} N^{j}}$. For $m=0$, the stress tensor becomes

$$
T_{i j}=\frac{1}{2 \pi^{2}} \sum_{\vec{n}} \frac{g_{i j} N^{2}-4 g_{i k} g_{j l} N^{k} N^{l}}{\left(g_{\mu \nu} N^{\mu} N^{\nu}\right)^{3}}, \quad \quad N^{2}=: g_{i j} N^{i} N^{j} .
$$

Another important case is a massless field on the equilateral torus for which $r_{1}=r_{2}=r_{3}$. The stress tensor takes the form announced above, i.e.

$$
T_{i j}=\frac{1}{3} \rho_{v}\left[g_{i j}+4 \xi_{i} \xi_{j}\right],
$$

where $\rho_{v}=\Omega_{3}^{-1} E_{v}$ is actually the limit $m \rightarrow 0$ in Eq. (4.3) and is also given by Eq. (4.5) by means of Epstein function (see Appendix D)

$$
\rho_{v}=-\frac{1}{2 \pi^{2}} \sum_{\vec{n}} \frac{1}{\left(g_{i j} N^{i} N^{j}\right)^{2}} .
$$

Hence, the (negative) vacuum energy density determines the stress tensor.

For the non-equilateral torus, one can compute the three principal pressures $P_{(i)}, i=1,2,3$. With a simple calculation we get (here the sum is excluded)

$$
P_{(i)}=-\rho_{v}-r_{i} \frac{\partial \rho_{v}}{\partial r_{i}},
$$

where $\rho_{v}=\Omega_{3}^{-1} A, A$ given by Eq. (4.4). Thus

$$
\rho_{v}-\sum_{i=1}^{3} P_{(i)} \geq 0
$$

and also in this case the energy density determines the stress tensor. We see that the above renormalization ansatz is equivalent to the $\zeta$-function prescription of removing the first term in Eq. (4.2). 


\subsection{The vacuum energy for massive scalar fields on $\mathbb{R} \times S^{N}$}

Now we review Einstein-like static universes, which have been extensively studied, for example, in Refs. [131, 132, 6]. As in the previous section, we consider an operator of the kind $L_{D}=$ $-\partial_{\tau}^{2}+\mathrm{E}_{N}$, but now $\mathrm{E}_{N}=-\Delta_{N}+\alpha^{2}+\kappa \varrho_{N}^{2}$ acts on fields in $S^{N}$. We have $\kappa \varrho_{N}^{2}=\kappa(N-1)^{2} / 4=$ $R(N-1) / 2 N$. We would like to compute vacuum energy $E_{v}^{S}\left(\mathrm{E}_{N}\right)$ for such systems. As a first step, we carry on the computation for the two cases in which the manifold is $\mathbb{R} \times S^{1}$ and $\mathbb{R} \times S^{2}$ with the assumption that $\alpha^{2}+\kappa \varrho_{N}^{2}$ is not vanishing (no zero-modes). Using Eqs. (2.34), (2.72), (C.2) and (3.18) we obtain the results

$$
\begin{aligned}
& E_{v}^{S}\left(\mathrm{E}_{1}\right)=-\frac{\nu \Omega_{1} \alpha^{2}}{4 \pi}\left(\ln \left(\ell^{2} \alpha^{2}\right)-1\right)-\frac{2 \nu \Omega_{1} \alpha^{2}}{\pi} \int_{1}^{\infty} \frac{\sqrt{u^{2}-1}}{e^{2 \pi \alpha r u}-1} d u, \\
& E_{v}^{S}\left(\mathrm{E}_{2}\right)=-\frac{\nu \Omega_{2} \alpha^{3}}{12} \int_{\Gamma}\left(1+\frac{|\kappa| z^{2}}{\alpha^{2}}\right)^{3 / 2} \frac{d z}{\cos ^{2} \pi z} .
\end{aligned}
$$

where in Eq. (4.6) $r$ is the radius of $S^{1}$.

When $\alpha^{2}+\kappa \varrho_{N}^{2}=0$, zero-modes are present. In this case, for $S^{1}$ we have to consider only the right hand side of Eq. (C.2), so that the $\zeta$-function becomes $2 r^{2 s} \zeta_{R}(2 s)$-function and the vacuum energy $E_{v}\left(-\Delta_{S^{1}}\right)=2 \nu \zeta_{R}(-1) / r$, while for $S^{2}$ we have to exclude the branch point $z=|\kappa|^{1 / 2} / 2$ from the path of integration in Eq. (4.7).

Using Eq. (3.15) in any $D+2$ dimensional manifold of the kind $\mathbb{R} \times S^{N+2}$ we get

$$
\frac{E_{v}^{S}\left(\mathrm{E}_{N+2}\right)}{\Omega_{N+2}}=-\frac{1}{2 \pi N \Omega_{N}}\left[\left(\alpha^{2}+\kappa \varrho_{N}^{2}\right) E_{v}^{S}\left(\mathrm{E}_{N}\right)-\nu \zeta^{(r)}\left(-3 / 2 \mid \mathrm{E}_{N}\right)\right] .
$$

Using Eq. 3.15) we also see that vacuum energies $E_{v}^{S}\left(\mathrm{E}_{N}\right)$ are determined by the values of $\zeta^{(r)}\left(s \mid \mathrm{E}_{1}\right)$ or $\zeta^{(r)}\left(s \mid \mathrm{E}_{2}\right)$ at the points $s=-n / 2$, with $n$ any odd integer smaller than or equal to $N$. Note that $\zeta\left(s \mid \mathrm{E}_{2}\right)$ has only a pole at $s=1$ and therefore (as we know) the vacuum energy does not need renormalization.

For the sake of completeness, we report also the expression of vacuum energy in $3+1$ dimensions. This is the Einstein static universe first treated in Ref. [131. It reads

$$
E_{v}^{S}\left(\mathrm{E}_{3}\right)=-\frac{\nu \Omega_{3} \alpha^{4}}{32 \pi^{2}}\left(\ln \left(\ell^{2} \alpha^{2}\right)-\frac{3}{2}\right)+\frac{\nu \Omega_{3} \alpha^{4}}{\pi^{2}} \int_{1}^{\infty} \frac{u^{2} \sqrt{u^{2}-1}}{e^{2 \pi \alpha|\kappa|^{-1 / 2} u}-1} d u .
$$

The renormalization procedure employed in Ref. [131] amounts, in the present treatment, to omit the first term in the formula. The renormalized stress tensor is again given by Eq. (4.1) with $\rho_{v}=\Omega_{3}^{-1} E_{R}$, where $E_{R}$ is the last term in Eq. (4.8).

\subsection{The vacuum energy for massive scalar fields on $\mathbb{R} \times H^{N} / \Gamma$}

This case has recently been studied in Refs. [133, 93, 134]. Here $\mathrm{E}_{N}=-\Delta_{N}+\alpha^{2}+\kappa \varrho_{N}^{2}$ acts on fields in $H^{N} / \Gamma$. Note that in contrast with the case treated above, now $R=N(N-1) \kappa$ is negative. First of all we concentrate on the contribution to vacuum energy due to the identity element of the group $\Gamma$. We shall indicate it by $E_{v}^{I}\left(\mathrm{E}_{N}\right)$. As a first step we carry on the computation for the two cases in which the manifold is $\mathbb{R} \times H^{3} / \Gamma$ and $\mathbb{R} \times H^{2} / \Gamma$ and then we extend the results to any dimension by using the recurrence relations (3.52).

In the first case we have the heat coefficients in a closed form and so, using Eqs. (2.34), (2.72) and (C.6) we obtain

$$
E_{v}^{I}\left(\mathrm{E}_{3}\right)=-\frac{\nu \Omega\left(\mathcal{F}_{3}\right) \alpha^{4}}{32 \pi^{2}}\left(\ln \left(\ell^{2} \alpha^{2}\right)-\frac{3}{2}\right)
$$


which differs from the analog expression in flat space-time for a modification of the mass term by the constant curvature. In the zeta-function treatment, the usual renormalization ansatz (see Ref. [135] and references therein) for the open Einstein universe amounts to remove the entire expression, thus getting a vanishing vacuum energy. The renormalized stress tensor is given by Eq. (4.1) with $\rho_{v}=0$, hence it is zero. The same conclusion is reached in Ref. [136].

In the second case, since the manifold without boundary has odd dimension, all odd coefficients of the heat kernel are vanishing. As a result, there is no $\ell$ ambiguity. Using Eq. (C.10), we get

$$
E_{v}^{I}\left(\mathrm{E}_{2}\right)=-\frac{\nu \Omega\left(\mathcal{F}_{2}\right) \alpha^{3}}{6} \int_{0}^{\infty}\left(1+\frac{|\kappa| r^{2}}{\alpha^{2}}\right)^{3 / 2} \frac{d r}{\cosh ^{2} \pi r} .
$$

Now, using Eq. (3.52) in any $D+2$ dimensional manifold of the kind $\mathbb{R} \times H^{N+2} / \Gamma$ we have

$$
\frac{E_{v}^{I}\left(\mathrm{E}_{N+2}\right)}{\Omega\left(\mathcal{F}_{N+2}\right)}=-\frac{1}{2 \pi N \Omega\left(\mathcal{F}_{N}\right)}\left[\left(\alpha^{2}+\kappa \varrho_{N}^{2}\right) E_{v}^{I}\left(\mathrm{E}_{N}\right)-\nu \zeta_{I}^{(r)}\left(-3 / 2 \mid \mathrm{E}_{N}\right)\right],
$$

from which we see that in order to compute the contribution to vacuum energy due to the identity of the group $\Gamma$, it is sufficient to know $\zeta\left(s \mid \mathrm{E}_{2}\right)$ and $\zeta\left(s \mid \mathrm{E}_{3}\right)$ at the negative half-integers.

The topological contribution due to hyperbolic elements only, can be easily expressed in terms of Selberg $\Xi$-function making use of Eq. (3.53). One has

$$
E_{v}^{H}\left(\mathrm{E}_{N}\right)=-\frac{\nu \alpha^{2}}{\pi|\kappa|^{1 / 2}} \int_{1}^{\infty} \sqrt{u^{2}-1} \Xi_{N}\left(u \alpha|\kappa|^{-1 / 2}+\varrho_{N}\right) d u
$$

Due to the properties of Selberg $\Xi$-function, this contribution to vacuum energy is always negative if one is dealing with untwisted fields, i.e. $\chi=1$ [93, 134].

Finally, in $4(N=3)$ and $3(N=2)$ dimensions we are able to evaluate also the contribution to vacuum energy due to elliptic elements of the $\Gamma$ isometry group. In fact, using Eqs. (C.8) and (C.11) we obtain 137]

$$
\begin{aligned}
& E_{v}^{E}\left(\mathrm{E}_{3}\right)=-\frac{\nu E \alpha^{2}}{4 \pi}\left(\ln \left(\ell^{2} \alpha^{2}\right)-\frac{1}{2}\right), \\
& E_{v}^{E}\left(\mathrm{E}_{2}\right)=4 \nu \alpha \int_{0}^{\infty} \sqrt{1+\frac{|\kappa| r^{2}}{\alpha^{2}}} E_{2}(r) d r
\end{aligned}
$$

and we see that the above renormalization ansatz is not sufficient to get rid of all the arbitrary scale dependences of the vacuum energy.

In the conformal case $(\alpha=0)$ the vacuum energy is trivially vanishing (in $3+1$-dimensions). Note however that if $\lambda=1$ is an eigenvalue of $\mathrm{E}_{3}$, the limit is trickier since the integrand in Eq. (4.9) has a simple pole just at $\alpha=0$.

\subsection{The self-interacting scalar field}

Here we concentrate our attention on a self-interacting scalar field defined on an ultrastatic space-time $\mathcal{M}^{\triangle}$ in which the spatial section is a manifold with constant curvature. For the sake of generality, we shall derive a general expression for the one-loop effective potential on such kind of space-times and then we shall discuss in detail the case in which the spatial section is $H^{3} / \Gamma$, the discrete group of isometries containing hyperbolic elements only. When $\Gamma$ contains elliptic elements, conical-like singularities appear in the manifold, and in this case the renormalization becomes quite delicate. For a discussion of similar situations see Ref. [138]. We analyze the possibility of symmetry breaking (or symmetry restoration) due to topology. We shall see that 
the sign of the square of the topological mass will depend on the character of the field, being positive for trivial character (untwisted fields-possible symmetry restoration). For twisted fields there could exist a mechanism for symmetry breaking. Here we shall use $\zeta$-function regularization but, as we already have pointed out, other regularizations are equally good.

We start with the classical Euclidean action for the scalar field

$$
S[\phi, g]=\int_{\mathcal{M}^{4}}\left[-\frac{1}{2} \phi \Delta \phi+V_{c}(\phi, R)\right] \sqrt{g} d^{4} x,
$$

where the classical potential reads

$$
V_{c}(\phi, R)=\frac{\lambda \phi^{4}}{24}+\frac{m^{2} \phi^{2}}{2}+\frac{\xi R \phi^{2}}{2} .
$$

From Eq. (4.10) we obtain the small disturbance operator

$$
L=-\Delta+V_{c}^{\prime \prime}\left(\phi_{c}, R\right)=-\Delta+m^{2}+\xi R+\frac{\lambda \phi_{c}^{2}}{2},
$$

the prime representing the derivative with respect to $\phi$.

Using $\zeta$-function regularization and Eq. (2.47), we write the one-loop quantum corrections to the effective potential in the convenient form

$$
\begin{aligned}
V^{(1)}\left(\phi_{c}, R\right) & =-\frac{1}{2 V(\mathcal{M})} \zeta^{\prime}\left(0 \mid L \ell^{2}\right) \\
& =\frac{1}{64 \pi^{2}}\left[2 a_{2} \ln \left(M^{2} \ell^{2}\right)-\frac{3}{2} M^{4}\right]+f\left(\phi_{c}, R\right),
\end{aligned}
$$

where $a_{2}$ is the spectral coefficient defined in Appendix B.1 and $M^{2}=-a_{1}$ is a function of (constant) $\phi_{c}$ and $R$. We have introduced the quantity

$$
f\left(\phi_{c}, R\right)=\frac{3 M^{4}}{128 \pi^{2}}-\frac{\zeta^{\prime}\left(0 \mid L / M^{2}\right)}{2 V(\mathcal{M})},
$$

which does not depend on the renormalization parameter $\ell$. Its utility shall become clear in the following.

Specializing Eqs. (B.1) and (B.2) to ultrastatic manifolds with constant curvature spatial section and recalling that, since we are computing the effective potential, $V(x)=V_{c}^{\prime \prime}\left(\phi_{c}, R\right)$ is a constant, we obtain

$$
V^{(1)}\left(\phi_{c}, R\right)=\frac{1}{64 \pi^{2}}\left[\left(M^{4}-\frac{c_{0} R^{2}}{2}\right) \ln \left(M^{2} \ell^{2}\right)-\frac{3}{2} M^{4}\right]+f\left(\phi_{c}, R\right),
$$

with

$$
M^{2}=V_{c}^{\prime \prime}\left(\phi_{c}, R\right)-\frac{R}{6}=m^{2}+\left(\xi-\frac{1}{6}\right) R+\frac{\lambda \phi_{c}^{2}}{2} .
$$

In this way, only $c_{0}$ and $f\left(\phi_{c}, R\right)$ depend on the chosen manifold. Note that only $R^{2}$ appears in the expression for $a_{2}$, since for the manifolds we are considering, all curvature invariants are proportional to $R$. For a more general treatment see Ref. [139].

It follows that the one-loop quantum corrections to the classical action generate quadratic terms in the curvature [45], so for the gravitational contribution we have to take the expression

$$
V_{g}=\Lambda+\alpha_{1} R+\frac{\alpha_{2} R^{2}}{2}
$$


and we must add to the classical potential a counterterm contribution, which reflects the structure of the one-loop divergences. It has the form

$$
\delta V\left(\phi_{c}, R\right)=\delta \Lambda+\frac{\delta \lambda \phi_{c}^{4}}{24}+\frac{\delta m^{2} \phi_{c}^{2}}{2}+\frac{\delta \xi R \phi_{c}^{2}}{2}+\delta \alpha_{1} R+\frac{\delta \alpha_{2} R^{2}}{2},
$$

$\Lambda \sim 0, \alpha_{1}$ and $\alpha_{2}$ being the cosmological and coupling constants respectively. As a result, the renormalized one-loop effective potential reads

$$
V_{e f f}=V_{c}+V_{g}+V^{(1)}+\delta V=V_{c}+V_{g}+V_{e f f}^{(1)} .
$$

When it will be convenient, we shall denote by $\delta \eta_{q} \equiv\left(\delta \lambda, \delta m^{2}, \delta \xi, \delta \alpha_{1}, \delta \alpha_{2}\right)$ the set of whole counterterm coupling constants.

\subsubsection{Renormalization conditions.}

The quantities $\delta \eta_{q}$, which renormalize the coupling constants, are determined by the following renormalization conditions (see for example Refs. [50, 140])

$$
\begin{array}{llrl}
\frac{\partial^{4} V_{\text {eff }}^{(1)}\left(\phi_{1}, R_{1}\right)}{\partial \phi_{c}^{4}}=0, & \frac{\partial V_{\text {eff }}^{(1)}(0,0)}{\partial R}=0, \\
\frac{\partial^{2} V_{\text {eff }}^{(1)}(0,0)}{\partial \phi_{c}^{2}}=0, & \frac{\partial^{2} V_{\text {eff }}^{(1)}\left(\phi_{5}, R_{5}\right)}{\partial R^{2}}=0, \\
\frac{\partial^{3} V_{\text {eff }}^{(1)}\left(\phi_{3}, R_{3}\right)}{\partial R \partial \phi_{c}^{2}}=0, & V_{\text {eff }}^{(1)}\left(\phi_{0}, 0\right)=0 .
\end{array}
$$

The latter equation is equivalent to say that the the cosmological constant is equal to $V_{c}\left(\phi_{0}, 0\right)$, $\phi_{0}=<\phi_{c}>$ being the mean value of the field. The choice of different points $\left(\phi_{c}, R\right) \equiv\left(\phi_{q}, R_{q}\right)$ in the renormalization conditions which define the physical coupling constants, is due to the fact that in general they are measured at different scales, the behaviour with respect to a change of scale being determined by the renormalization group equations (2.53), discussed in Sec. 2.4. By a suitable choice of $\left(\phi_{q}, R_{q}\right)$, Eqs. (4.12) reduce to the conditions chosen by other authors (see for example Refs. [50, 140]).

From such conditions, after straightforward calculations we get the counterterms

$$
\begin{array}{r}
64 \pi^{2}\left(\delta \Lambda+f\left(\phi_{0}, 0\right)\right)=64 \pi^{2}\left[\left(\frac{\partial^{2} f(0,0)}{\partial \phi_{c}^{2}}-m^{2}\right) \frac{\phi_{0}^{2}}{2}+\left(\frac{\partial^{4} f\left(\Phi_{1}\right)}{\partial \phi_{c}^{4}}-\lambda\right) \frac{\phi_{0}^{4}}{24}\right] \\
-\lambda m^{2} \phi_{0}^{2}\left[1-\ln \left(m^{2} \ell^{2}\right)\right]+M_{0}^{4}\left[\frac{3}{2}-\ln \left(M_{0}^{2} \ell^{2}\right)\right] \\
+\frac{\lambda^{2} \phi_{0}^{4}}{4} \ln \left(M_{1}^{2} \ell^{2}\right)-\frac{\lambda^{4} \phi_{0}^{4} \phi_{1}^{4}}{12 M_{1}^{4}}+\frac{\lambda^{3} \phi_{0}^{4} \phi_{1}^{2}}{2 M_{1}^{2}}, \\
64 \pi^{2}\left[\delta \lambda+\frac{\partial^{4} f\left(\phi_{1}, R_{1}\right)}{\partial \phi_{c}^{4}}\right]=-6 \lambda^{2} \ln \left(M_{1}^{2} \ell^{2}\right)+\frac{2 \lambda^{4} \phi_{1}^{4}}{M_{1}^{4}}-\frac{12 \phi_{1}^{2} \lambda^{3}}{M_{1}^{2}}, \\
64 \pi^{2}\left[\delta m^{2}+\frac{\partial^{2} f(0,0)}{\partial \phi_{c}^{2}}\right]=2 \lambda m^{2}\left[1-\ln \left(m^{2} \ell^{2}\right)\right],
\end{array}
$$




$$
\begin{gathered}
64 \pi^{2}\left[\delta \xi+\frac{\partial^{3} f\left(\phi_{3}, R_{3}\right)}{\partial R \partial \phi_{c}^{2}}\right]=-2 \lambda\left(\xi-\frac{1}{6}\right) \ln \left(M_{3}^{2} \ell^{2}\right)-\frac{2 \lambda^{2}\left(\xi-\frac{1}{6}\right) \phi_{3}^{2}}{M_{3}^{2}} \\
+\frac{c_{0} \lambda R_{3}^{2}}{M_{3}^{2}}\left(1-\frac{\lambda \phi_{3}^{2}}{M_{3}^{2}}\right), \\
64 \pi^{2}\left[\delta \eta_{4}+\frac{\partial f(0,0)}{\partial R}\right]=2 m^{2}\left(\xi-\frac{1}{6}\right)\left[1-\ln \left(m^{2} \ell^{2}\right)\right], \\
64 \pi^{2}\left[\delta \eta_{5}+\frac{\partial^{2} f\left(\phi_{5}, R_{5}\right)}{\partial R^{2}}\right]=-\left[2\left(\xi-\frac{1}{6}\right)^{2}-c_{0}\right] \ln \left(M_{5}^{2} \ell^{2}\right)+\frac{2\left(\xi-\frac{1}{6}\right) c_{0} R_{5}^{2}}{M_{5}^{2}},
\end{gathered}
$$

where we introduced the constants $M_{n}^{2}=m^{2}+\left(\xi-\frac{1}{6}\right) R_{n}+\frac{\lambda}{2} \phi_{n}^{2}$.

The renormalized one-loop contribution to the effective potential looks very complicated. We write it in the form

$$
\begin{aligned}
& 64 \pi^{2} V_{e f f}^{(1)}=-32 \pi^{2} m^{2} \phi_{0}^{2}-\frac{8 \pi^{2} \lambda \phi_{0}^{4}}{3}+\lambda m^{2} \phi_{0}^{2}\left(\ln \frac{m^{2}}{M_{0}^{2}}+\frac{1}{2}\right) \\
&-\frac{\lambda^{2} \phi_{0}^{4}}{4}\left[\ln \frac{M_{0}^{2}}{M_{1}^{2}}-\frac{3}{2}-\frac{4\left(M_{1}^{2}-m^{2}\right)\left(2 M_{1}^{2}+m^{2}\right)}{3 M_{1}^{4}}\right] \\
&+m^{4} \ln \frac{M^{2}}{M_{0}^{2}}+2 m^{2}\left(\xi-\frac{1}{6}\right) R\left(\ln \frac{M^{2}}{m^{2}}-\frac{1}{2}\right)+\left(\xi-\frac{1}{6}\right)^{2} R^{2}\left(\ln \frac{M^{2}}{M_{5}^{2}}-\frac{3}{2}\right) \\
&+\left\{\left(\xi-\frac{1}{6}\right) R\left[\ln \frac{M^{2}}{M_{3}^{2}}-\frac{3}{2}-\frac{\lambda \phi_{3}^{2}}{M_{3}^{2}}\right]+m^{2}\left[\ln \frac{M^{2}}{m^{2}}-\frac{1}{2}\right]\right\} \lambda \phi_{c}^{2} \\
&+\left.\left.\left\{\left(\ln \frac{M^{2}}{M_{1}^{2}}-\frac{25}{6}\right)+\frac{4 m^{2}\left(m^{2}+M_{1}^{2}\right)}{3 M_{1}^{4}}\right\} \frac{\lambda^{2} \phi_{c}^{4}}{4} M^{2}-\frac{2\left(\xi-\frac{1}{6}\right) R_{5}^{2}}{M_{5}^{2}}\right] \frac{R^{2}}{2}\right\} \\
&-c_{0}\left\{\frac{\lambda R_{3}^{2}}{2 M_{3}^{2}}\left(\frac{\lambda \phi_{3}^{2}}{M_{3}^{2}}-1\right) R+\left[\ln \frac{M^{2}}{M_{5}^{2}}-\frac{1}{+}\right.\right. \\
&+ 64 \pi^{2} F(f),
\end{aligned}
$$

where $F(f)$ contains all terms depending on $f\left(\phi_{c}, R\right)$. It reads

$$
\begin{aligned}
F(f)=f\left(\phi_{c}, R\right) & -f\left(\phi_{0}, 0\right)+\frac{\partial^{4} f\left(\phi_{1}, R_{1}\right)}{\partial \phi_{c}^{4}} \frac{\phi_{c}^{4}-\varphi_{0}^{4}}{24}+\frac{\partial^{2} f(0,0)}{\partial \phi_{c}^{2}} \frac{\phi_{c}^{2}-\varphi_{0}^{2}}{2} \\
& -\frac{\partial^{3} f\left(\phi_{3}, R_{3}\right)}{\partial \phi_{c}^{2} \partial R} \frac{R \phi_{c}^{2}}{2}-\frac{\partial f(0,0)}{\partial R} R-\frac{\partial^{2} f\left(\phi_{5}, R_{5}\right)}{\partial R^{2}} \frac{R^{2}}{2}
\end{aligned}
$$

We see that the computation of the one-loop effective Lagrangian reduces to the determination of the function $f\left(\phi_{c}, R\right)$.

If we consider a flat space-time and take the limit $m \rightarrow 0$, of course we get the ColemanWeinberg result 43.

$$
V_{e f f}=\frac{\lambda \phi_{c}^{4}}{24}+\frac{\lambda^{2} \phi_{c}^{4}}{256 \pi^{2}}\left(\ln \frac{\lambda \phi_{c}^{2}}{2 M_{1}^{2}}-\frac{25}{6}\right) .
$$

What is relevant for the discussion of the phase transition of the system, is the second derivative of $V_{\text {eff }}$ with respect to the background field $\phi_{c}$. Then we define

$$
V_{e f f}=\Lambda_{e f f}(R)+\frac{m_{e f f}^{2}(R) \phi_{c}^{2}}{2}+O\left(\phi_{c}^{4}\right),
$$


where $\Lambda_{\text {eff }}(R)$ and $m_{\text {eff }}^{2}(R)$ are complicated expressions not depending on $\phi_{c}$. They contain curvature and topological contributions to $\Lambda$ and $m^{2}$. By a straightforward computation we get

$$
\begin{aligned}
m_{\text {eff }}^{2}= & m^{2}+\xi R \\
& +\frac{\lambda}{32 \pi^{2}}\left\{m^{2} \ln \frac{m^{2}+\left(\xi-\frac{1}{6}\right) R}{m^{2}}+\left(\xi-\frac{1}{6}\right) R\left[\ln \frac{m^{2}+\left(\xi-\frac{1}{6}\right) R}{M_{3}^{2}}-\frac{\lambda \phi_{3}^{2}}{M_{3}^{2}}-1\right]\right\} \\
& -\frac{\lambda c_{0} R^{2}}{128 \pi^{2}\left[m^{2}+\left(\xi-\frac{1}{6}\right) R\right]}+\frac{\partial^{2} f(0, R)}{\partial \phi_{c}^{2}}-\frac{\partial^{2} f(0,0)}{\partial \phi_{c}^{2}}-\frac{\partial^{3} f\left(0, R_{3}\right)}{\partial \phi_{c}^{2} \partial R} R
\end{aligned}
$$

When $\phi_{c}=0$ is a minimum for the classical potential, Eqs. (4.14) and (4.15) are useful in the discussion of phase transition.

\subsubsection{The ultrastatic space-time $\mathbb{R} \times H^{3} / \Gamma$.}

For this case we shall give a detailed treatment and in particular, we shall analyze the possibility of symmetry breaking due to topology. At the end of the section, other examples shall be briefly discussed.

For these kind of manifolds we have $c_{0}=0\left(a_{2}=M^{4} / 2\right)$ and moreover, as one can easily check by using Eqs. (2.35) and (C.10), the identity of the isometry group $\Gamma$ does not give contributions to the function $f\left(\phi_{c}, R\right)$. This means that it has only a topological contribution given by

$$
V_{\text {top }}\left(\phi_{c}, R\right)=f\left(\phi_{c}, R\right)=-\frac{M^{2}|\kappa|^{-1 / 2}}{2 \pi \Omega\left(\mathcal{F}_{3}\right)} \int_{1}^{\infty} \sqrt{u^{2}-1} \Xi_{3}\left(1+u M|\kappa|^{-1 / 2}\right) d u,
$$

where we have used Eqs. (2.35) and Eq. (3.53) with $\alpha=M$. The latter equation represents the unrenormalized topological contribution to the one-loop effective potential. Using the properties of the $\Xi$-function one can see that for $M>0, V_{\text {top }}$ is exponentially vanishing when $\kappa=R / 6$ goes to zero. Then, from Eq. (4.13) we obtain

$$
\begin{aligned}
F(f)=V_{\text {top }}\left(\phi_{c}, R\right) & +\frac{\partial^{4} V_{\text {top }}\left(\phi_{1}, R_{1}\right)}{\partial \phi_{c}^{4}}\left(\frac{\phi_{c}^{4}-\phi_{0}^{4}}{24}\right) \\
& -\frac{\partial^{3} V_{t o p}\left(\phi_{3}, R_{3}\right)}{\partial \phi_{c}^{2} \partial R} \frac{R \phi_{c}^{2}}{2}-\frac{\partial^{2} V_{t o p}\left(\phi_{5}, R_{5}\right)}{\partial R^{2}} \frac{R^{2}}{2}
\end{aligned}
$$

and this is the renormalized topological contribution to the one-loop effective potential. We also have from Eq. (4.15)

$$
\begin{aligned}
m_{\text {eff }}^{2}=m^{2}+\xi R+m_{\text {top }}^{2}+ & \frac{\lambda}{32 \pi^{2}}\left\{m^{2} \ln \frac{m^{2}+\left(\xi-\frac{1}{6}\right) R}{m^{2}}\right. \\
& \left.+\left(\xi-\frac{1}{6}\right) R\left[\ln \frac{m^{2}+\left(\xi-\frac{1}{6}\right) R}{M_{3}^{2}}-\frac{\lambda \phi_{3}^{2}}{M_{3}^{2}}-1\right]\right\},
\end{aligned}
$$

where the topological contribution $m_{\text {top }}^{2}$ has been introduced. To simplify the discussion, from now on we choose $R_{1}=R_{3}=R_{5}=0$ like in Ref. [140. In this way $m_{\text {top }}^{2}$ assumes the form

$$
m_{\text {top }}^{2}=\frac{\sqrt{3} \lambda|R|^{-1 / 2}}{4 \pi \Omega\left(\mathcal{F}_{3}\right)} \int_{1}^{\infty} \Xi_{3}\left(1+u \sqrt{6 m^{2}|R|^{-1}+1-6 \xi}\right) \frac{d u}{\sqrt{u^{2}-1}} .
$$


The symmetry breaking. For the discussion on the physical implications of the one-loop effective potential, let us specialize to different cases and to several limits.

Let us first concentrate on the regime $m^{2}+\xi R>0$. Then $\phi_{c}=0$ is a minimum of the classical potential and we can use expansion (4.14) to carry on the analysis. We consider the small and large curvature limits separately. As mentioned before, the topological contribution is negligible when $R \rightarrow 0$ and so the leading orders to $m_{\text {eff }}^{2}$ only including linear curvature terms are easily obtained from Eq. (4.15). We have

$$
m_{e f f}^{2}(R)=m^{2}+\xi R-\frac{\lambda\left(\xi-\frac{1}{6}\right) R}{32 \pi^{2}}\left(\frac{\lambda \phi_{3}^{2}}{M_{3}^{2}}-\ln \frac{m^{2}}{M_{3}^{2}}\right) .
$$

This result is effectively true for any smooth Riemannian manifold. Due to $R<0$ in our example, for $\xi<\frac{1}{6}$ the one loop term will help to break symmetry, whereas for $\xi>\frac{1}{6}$ the quantum contribution acts as a positive mass and helps to restore symmetry. The quantum corrections should be compared to the classical terms which in general dominate, because the one loop terms are suppressed by the square of the Planck mass. But if $\xi$ is small enough the one loop term will be the most important and will then help to break symmetry.

Let us now concentrate on the opposite limit, that is $|R| \rightarrow \infty$ with the requirement $\xi<0$. In that range the leading order of the topological part reads

$$
m_{\text {top }}^{2}=\frac{\sqrt{3} \lambda|R|^{-1 / 2}}{4 \pi \Omega\left(\mathcal{F}_{3}\right)} \int_{1}^{\infty} \Xi_{3}(1+u \sqrt{1-6 \xi}) \frac{d u}{\sqrt{u^{2}-1}}+O\left(R^{0}\right) .
$$

It is linear in the scalar curvature $R$ because $\Omega\left(\mathcal{F}_{3}\right) \sim|R|^{-3 / 2}$. The sign of the contribution depends on the choice of the characters $\chi(\gamma))$. For trivial character $\chi=1$ we can say that the contribution helps to restore symmetry, whereas for nontrivial character it may also serve as a symmetry breaking mechanism. So, if the symmetry is broken at small curvature, for $\chi=1$ a symmetry restoration at some critical curvature $R_{c}$, strongly depending on the non trivial topology, will take place.

Finally, let us say some words about the regime $m^{2}+\xi R<0$, which includes for example the conformal invariant case. The classical potential has two minima at $\pm \sqrt{6\left|m^{2}+\xi R\right| / \lambda}$. So even for $\xi>0$, due to the negative curvature in the given space-time, the classical starting point is a theory with a broken symmetry. As is seen in the previous discussion, in order to analyze the influence of the quantum corrections on the symmetry of the classical potential, a knowledge of the function $\Xi_{3}(s)$ for values $\operatorname{Re} s<2$ is required. But $\Xi_{3}(s)$ has a simple pole at $s=2$ [123]. As may then be seen in equation (4.16), the topological contribution contains a part which behaves like $\sqrt{m^{2}+\xi R}$ near $m^{2}+\xi R=0$ (resulting from the range of integration near $u=1$ ). So for $m^{2}+\xi R<0$ the effective potential becomes complex, which reflects the well known failure of the loop expansion in this range of parameters [70, 141].

Some remarks. In new inflationary models, the effective cosmological constant is obtained from an effective potential, which includes quantum corrections to the classical potential of a scalar field [43]. This potential is usually calculated in Minkowski space-time, whereas to be fully consistent, the effective potential must be calculated for more general space-times. For that reason an intensive research has been dedicated to the analysis of the one-loop effective potential of a self-interacting scalar field in curved space-time. Especially to be mentioned are the considerations on the torus [142, 143, 144, 145, 146], which however do not include nonvanishing curvature, and the quasi-local approximation scheme developed in Ref. [50] (see also Ref. [147]), which however fails to incorporate global properties of space-time. To overcome this deficiency, we were naturally led to the given considerations on the space-time manifold $\mathbb{R} \times H^{3} / \Gamma$. Apart from its physical relevance [106], this manifold combines nonvanishing curvature with highly 
nontrivial topology, still permitting the exact calculation of the one-loop effective potential by the use of the Selberg trace formula. So, at least for $m^{2}+\xi R>0$ and trivial line bundles $(\chi=1)$, we were able to determine explicitly the influence of the topology, namely the tendency to restore the symmetry. Furthermore, this contribution being exponentially damped for small curvature, we saw, in that regime, that for $\xi<\frac{1}{6}$ the quantum corrections to the classical potential can help to break symmetry.

\subsubsection{The ultrastatic space-times $\mathbb{R} \times T^{3}, \mathbb{R} \times S^{3}, \mathbb{R}^{2} \times S^{2}, \mathbb{R}^{2} \times H^{2} / \Gamma$}

For the sake of completeness we shall consider also these examples, but for all of them we shall simply give the value of $c_{0}$ and the explicit expression of the function $f\left(\phi_{c}, R\right)$, which is what one needs for the evaluation of $V_{\text {eff }}$.

Example: $\mathbb{R} \times T^{3}$. For simplicity we consider an equilateral torus $T^{3}$ with radius $r . \mathcal{M}^{\triangle}$ is a flat manifold and so we have $R=0$ in all formulae. Using Eqs. (2.35) and (3.4) for $f\left(\phi_{c}\right)$ we get

$$
f\left(\phi_{c}\right)=\frac{M^{4}}{3 \pi^{2}} \int_{1}^{\infty} \sqrt{u^{2}-1} \sum_{\vec{n} \in \mathbb{Z}^{3}, \vec{n} \neq 0} e^{-2 \pi M r|\vec{n}| u} d u
$$

Example: $\mathbb{R} \times S^{3}$. It is easy to see that if the spatial section is a maximally symmetric space, then $a_{2}=M^{4} / 2$, that is $c_{0}=0$. Again, using Eqs. (2.35) and (C.3) with $\alpha=M>0$ we obtain

$$
f\left(\phi_{c}, R\right)=\frac{M^{4}}{2 \pi^{2}} \int_{1}^{\infty} \frac{u^{2} \sqrt{u^{2}-1}}{e^{2 \pi M|\kappa|^{-1 / 2} u}-1} d u
$$

where $\kappa=R / 6$.

Example: $\mathbb{R}^{2} \times S^{2}$. For this case we have $c_{0}=1 / 180$. Using Eqs. (2.35) and (C.4) with $\alpha^{2}=M^{2}+\kappa / 12=M^{2}+R / 24$, we get

$$
\begin{aligned}
& f\left(\phi_{c}, R\right)=\frac{1}{64 \pi^{2}}\left\{-\frac{\kappa^{2}}{30}+\pi M^{4}\right. \int_{0}^{\infty}\left[1-\frac{\kappa\left(u^{2}-1 / 12\right)}{M^{2}}\right]^{2} \\
&\left.\times \ln \left|1-\frac{\kappa\left(u^{2}-1 / 12\right)}{M^{2}}\right| \frac{d u}{\cosh ^{2} \pi u}\right\} .
\end{aligned}
$$

Example: $\mathbb{R}^{2} \times H^{2} / \Gamma$. We again have $c_{0}=1 / 180$. Using Eqs. (2.35) and (C.10) with $\alpha^{2}=M^{2}+\kappa / 12=M^{2}+R / 24$, the identity contribution is given by Eq. (4.19), however now $\kappa<0$. If the isometry group $\Gamma$ contains only hyperbolic elements, then topological contributions can be easily computed using Eq. (3.53). As a result

$$
\begin{aligned}
& f\left(\phi_{c}, R\right)= \frac{1}{64 \pi^{2}}\left\{-\frac{\kappa^{2}}{30}+\pi M^{4} \int_{0}^{\infty}\left[1-\frac{\kappa\left(u^{2}-1 / 12\right)}{M^{2}}\right]^{2}\right. \\
&\left.\times \ln \left|1-\frac{\kappa\left(u^{2}-1 / 12\right)}{M^{2}}\right| \frac{d u}{\cosh ^{2} \pi u}\right\} \\
&-\frac{\left(M^{2}+k / 12\right)^{3 / 2}|\kappa|^{-1 / 2}}{8 \pi \Omega\left(\mathcal{F}_{2}\right)} \int_{1}^{\infty}\left(u^{2}-1\right) \Xi_{2}\left(\frac{1}{2}+u \sqrt{\frac{M^{2}}{\kappa}+\frac{1}{12}}\right) d u .
\end{aligned}
$$




\section{Quantum $p$-branes in curved space-times}

It is well known the great interest that has been recently arisen in regarding string theory as the Theory of Everything, including possibly also the gravitational interaction [4]. In spite of his successes, soon after it has been proposed a generalization to higher dimensional extended objects, the $p$-branes (strings for $p=1$, membranes for $p=2$, etc.).

There are several reasons for considering relativistic extended objects or $p$-branes $(p>2)$. To begin with, they are the natural generalizations of strings. Furthermore the study of (super)pbranes is a way to better understand, in particular, (super)strings. By the simultaneous reduction of the world-volume and space-time of a supermembrane model (modified in an appropriate way in order to account for the 11-dimensional supergravity background) it is possible to derive the type II A superstring in 10-dimensions [148]. Furthermore, the field theory limit of the underlying string theory is just the supergravity theory which links string and particle physics at low energy. In fact $(\mathrm{D}=11, \mathrm{~N}=1)$-supergravity cannot be obtained from string theories, but it can be obtained from suitable $p$-brane models. These are some of the reasons advocated in starting the study of these higher dimensional extended objects.

The interest in the quantum theory of (super)p-branes, has also been motivated by their mathematical structure as well as by their possible significance for the unification of fundamental interactions. One of the central issues is the nature of the mass spectrum in the effective low energy limit. In order to investigate the massive state one needs the knowledge of the quantum properties of the theory. Unfortunately, within the quantum theory of $p$-branes, there exist many unsolved questions up to now. The main difficulty is a consequence of the inherent non-linearity of the field equations as well as the related quantization of the theory.

In the following, we shall discuss some aspects of quantization of extended objects. These brief considerations do not claim to be a complete description and the aspects of the quantization which will be considered, reside mainly in the interests of the authors. As far as the issue of quantization is concerned, we say again that the main problems come from the non-linearity of $p$-branes models as well as the non-renormalizability in $(p+1)$-dimensions. Also the connection between the dimension of the embedding space $(D)$ and the quantum consistency has to be stressed. We shall aside the problem of the renormalizability and we shall concentrate only on some aspects of the quantizations of these systems, which have some similarity with string theory.

\subsection{Classical properties of (super) $p$-branes}

To begin with we recall some properties of $p$-branes. There are several proposals for the $p$-brane action, which are equivalent at the classical level. The most popular among them are the Dirac [158], Howe-Tucker [159] and conformally invariant actions [153, 160] which look as follows

$$
\begin{aligned}
& S_{D}=k \int\left[-\operatorname{det}\left(\partial_{i} X^{\mu} \partial_{j} X^{\nu} g_{\mu \nu}\right)\right]^{\frac{1}{2}} d^{p+1} \xi \\
& S_{H T}=\frac{k}{2} \int \sqrt{|\gamma|}\left[\gamma^{i j} \partial_{i} X^{\mu} \partial_{j} X^{\nu} g_{\mu \nu}-(p-1)\right] d^{p+1} \xi \\
& S_{C}=k \int \sqrt{|\gamma|}\left(\frac{1}{p+1} \gamma^{i j} \partial_{i} X^{\mu} \partial_{j} X^{\nu} g_{\mu \nu}\right)^{\frac{p+1}{2}} d^{p+1} \xi
\end{aligned}
$$

respectively. Here $k$ is the $p$-brane tension parameter with dimensions of $(\text { mass })^{p+1}$ and $\gamma$ is the determinant of the world metric $\gamma_{i j}=\partial_{i} X^{\mu} \partial_{j} X^{\nu} g_{\mu \nu}$. The indices $i, j, \ldots$ label the coordinates 
$\xi^{i}$ on the world volume (one time-like, $p$ space-like) and the fields $X^{\mu}(\mu=0, \ldots, D-1)$ are the coordinates of space-time. The equations of motion derived from action (5.1) are

$$
\partial_{i}\left(\sqrt{\gamma} \gamma^{i j} \partial_{j} X^{\nu} g_{\mu \nu}\right)=\frac{1}{2} \sqrt{\gamma} \gamma^{i j} \partial_{i} X^{\nu} \partial_{j} X^{\lambda} \frac{\partial g_{\nu \lambda}}{\partial X^{\mu}}, \quad \gamma^{i m} \gamma_{j m}=\delta_{j}^{i}
$$

The Dirac action enjoys general coordinate (reparametrization) invariance. Therefore the Hamiltonian of the model is a linear combination of the $(p+1)$ first class constraints 161

$$
H_{T}=P^{\mu} P_{\mu}+k^{2} G, \quad H_{i}=P_{\mu} \partial_{i} X^{\mu},
$$

where $G$ is the determinant of the space-like part of the metric $\gamma_{i j}$.

For the Howe-Tucker action (5.2) we have equations equivalent to the above ones. It is clear that the world volume cosmological constant $\Lambda=p-1$ vanishes only for the special case $p=1$. This means that only the string action is invariant under the conformal symmetry. Finally, the equations of motion generated by the conformally invariant action (5.3) are

$$
\begin{gathered}
(p+1) \sqrt{\gamma} \gamma^{i j}\left(\gamma^{k l} \partial_{k} X^{\mu} \partial_{l} X^{\nu} g_{\mu \nu}\right)^{\frac{p+1}{2}}= \\
\left(\gamma^{k l} \partial_{k} X^{\mu} \partial_{l} X^{\nu} g_{\mu \nu}\right)^{\frac{p-1}{2}} \sqrt{\gamma} \gamma^{i m} \gamma^{j n} \partial_{m} X^{\rho} \partial_{n} X^{\sigma} g_{\rho \sigma}, \\
\partial_{i}\left(\sqrt{\gamma}\left(\gamma^{k l} \partial_{k} X^{\mu} \partial_{l} X^{\sigma} g_{\mu \sigma}\right)^{\frac{p-1}{2}} \gamma^{i j} \partial_{j} X^{\nu} g_{\mu \nu}\right)= \\
\frac{\sqrt{\gamma}}{2}\left(\frac{\gamma^{k l}}{p+1} \partial_{k} X^{\rho} \partial_{l} X^{\sigma} g_{\rho \sigma}\right)^{\frac{p-1}{2}} \gamma^{i j} \partial_{i} X^{\nu} \partial_{j} X^{\lambda} \frac{\partial g_{\nu \lambda}}{\partial X^{\mu}} .
\end{gathered}
$$

In fact, the latter equations are equivalent to the other two ones provided that $\gamma_{i j}$ is a function of the space-time parameter $\xi^{i}$, namely $\gamma_{i j}=F\left(\xi^{i}\right)$ 160.

A novel $p$-brane action From the above discussion, one can see that there are several reasonable generalizations of the string action. We only mention the Lagrangian for an extended $p$-dimensional object which has been proposed in Ref. 162]. Another example will be discussed here and we propose it only for the sake of completeness. The idea is to generalize to extended objects the following reparametrization invariant action for a relativistic point particle (Euclidean signature):

$$
S=\int\left(\frac{g_{\mu \nu} \dot{X}^{\mu} \dot{X}^{\nu}}{2 \dot{f}}+\frac{m^{2} \dot{f}}{2}\right) d \xi .
$$

Note that in contrast with the well known einbein action [163, where the einbein plays the role of a Lagrangian multiplier, here the additional degree of freedom is represented by means of an auxiliary field $f$, appearing at the same level of the coordinates $X^{\mu}$. Note that the the extended object version of the einbein action is given by the Polyakov-Howe-Tucker action. Coming back to action (5.4), the conjugate momentum of the variable $f$ must vanish. As a result, making use of the equation of motion, the above action reduces to the familiar square root action. Note also that the reparametrization invariance of the action is a direct consequence of the structure of the Lagrangian, since it is a homogeneous function of degree one in the velocities.

For an extended object the natural generalization of the above action requires an enlargement of the configuration space, namely $\left(X^{\mu}, f^{a}\right)$, with $a=0,1,2, \ldots p$. The $p+1$ additional "coordinates " $f^{a}$, are scalar functions. Therefore one may write down an action which is linear in $\gamma$ and which is a generalization of Schild string action 164

$$
S=\int\left[\frac{\gamma}{2 F}+\frac{k^{2} F}{2} d^{p+1} \xi\right],
$$


with $\gamma$ as above $f \equiv\left(f^{0}, f^{1}, \ldots f^{p}\right)$ and $F=\left|\frac{\partial f}{\partial \xi}\right|$. The presence of the Jacobian $F$ makes the Lagrangian a scalar density of weight $-1 / 2$, rendering the action reparametrization invariant.

A classical canonical analysis can be done, but we stop here because we shall be interested in path integral quantization. Here, we shall limit ourselves only to few remarks. The conjugate momenta associated with $X_{\mu}$ and $f^{a}$ are respectively

$$
\begin{aligned}
& P_{\mu}=\frac{\partial L}{\partial \dot{X}_{\mu}}=\frac{\gamma}{F} \gamma^{m 0} \partial_{m} X_{\mu}, \\
& \pi_{a}=\frac{\partial L}{\partial \dot{f}^{a}}=\left(-\frac{\gamma}{2 F^{2}}+\frac{k^{2}}{2}\right)\left|\frac{\partial f}{\partial \xi}\right|_{a},
\end{aligned}
$$

where the time-like variable $f^{0}$ and the generic coordinates $\xi^{a}$ are missing in the Jacobian. At $F^{2}=\gamma k^{-2}$, we obtain $p+1$ primary constraints. Further, for $j \neq 0$ and taking Eq. (5.6) into account, we obtain further $p$ constraints $\partial_{j} X^{\mu} P_{\mu}=0$. As a result, we arrive at the secondary Hamiltonian constraint $P^{2}+k^{2} G=0$. Since the Lagrangian density is a homogeneous function of degree one in the velocities, the canonical Hamiltonian is identically vanishing. This is a general feature of theories which are reparametrization invariant. A direct calculation shows that all the constraints we have obtained are identically preserved in time. As a consequence there are no other constraints and the number of independent degrees of freedom is given by the number of initial Lagrangian coordinates $D+p+1$ minus the number of constraints which are $2 p+2$. As a result we have $D-p-1$ independent degrees of freedom. Furthermore, the constraint algebra of the action (5.5) and the Dirac action are the same and therefore describe the same physics. It should be noted that the action (5.5) allows one to take the limit $k$ goes to zero and permits the study of null $p$-branes which are the higher dimensional counterpart of massless particles.

\subsection{Some remarks on $p$-brane quantization}

We have shown that at a classical level the $p$-brane theory has some problems. In fact, the action of the theory contains a world-volume cosmological constant (the cosmological term) and this constant is vanishing only for $p=1$. As a consequence only the string action is invariant under conformal symmetry. In Ref. 165 an alternative bosonic $p$-brane action with an independent world volume metric and without the cosmological term has been proposed, but difficulties associated with the corresponding tensor calculus are present in this model, even for $p=2$.

We shall briefly consider later the path integral quantization (or Polyakov approach [166]). Here we only limit ourselves to some remarks. One issue is the difficulty related to the connection between any topological characterization of $(p+1)$-manifolds (largely incomplete) and loop diagrams. Another one is the generalization of (super)strings geometrical quantization [167, 168] based on the reparametrization invariance (super)diff $S^{1} / S^{1}$. In a pionering work 169, Hoppe has showed that the algebra diff: $S^{2} / S^{2}$, in the limit $N \rightarrow \infty$ is isomorphic to $S U(N)$. In the linear approximation the simplest possibility is to consider $p$-branes with the topology of the $p$-torus.

The supersymmetric version of Hoppe construction is contained in [170]. The gauge theory of the area-preserving diffeomorphisms of the supermembrane can be obtained as a limit of supersymmetric quantum mechanics. In contrast with the perturbative approach, massless modes are absent in the spectrum of the 11-dimensional supermembrane.

The BRST formalism for bosonic $p$-branes has been developed in [171]. The covariant BRST quantization of the Green-Schwartz superstring has been carried out in [172, 173] and investigations along these lines for $p>1$ are in progress. With regard to the connection between $D$ and the absence of anomalies (quantum consistency), it is well known that for string $D=26$ and for 
super-string $D=10$. In Ref. [174], it is claimed that for the bosonic membrane, $D=27$ is necessary for the quantum consistency (see however Ref. [175]). We also would like to mention the quantization scheme based on $p$-volume functionals [176]. Within this approach, there exists the possibility to find a functional diffusion equation in which the measure of the $p$-volume plays the role analogue of the proper time in the point particle dynamics [177, 178, 179]. This formalism has been introduced for the bosonic string in Refs. [180, 181] and for any $p$ in Ref. [182]. This issue is related to the path integral quantization of Sec. 5.5.

Now we shall consider the supersymmetric $p$-brane. The non-linear action for the supermembrane in 11-dimensional flat space-time may be written as [148]

$$
S=-\int d^{3} \xi\left[\sqrt{-g}\left(g^{i j} \Pi_{i}^{\mu} \Pi_{j \mu}-1\right)+2 \varepsilon^{i j k} \Pi_{i}^{A} \Pi_{j}^{B} \Pi_{k}^{C} B_{C B A}\right] .
$$

Here the first term is the straightforward supersymmetrization of the action for a bosonic membrane, while the second term may be conveniently understood as a Wess-Zumino-Witten term

$$
\Pi_{i}^{A}=\left(\Pi_{i}^{\mu}, \Pi_{i}^{\alpha}\right), \quad \Pi_{i}^{\mu}=\partial_{i} X^{\mu}-i \bar{\psi} \Gamma^{\mu} \partial_{i} \psi, \quad \Pi_{i}^{\alpha}=\partial_{i} \psi^{\alpha} .
$$

Our conventions are: $X^{\mu}$ is the bosonic variable $(\mu=0,1, \ldots, 10), \psi^{\alpha}$ is a 32-component Majorana spinor, the tension membrane is equal to unity, $\left\{\Gamma^{\mu}, \Gamma^{\nu}\right\}=-2 \eta^{\mu \nu}$ with $\eta^{\mu \nu}=\operatorname{diag}(-1,1, \ldots, 1)$, $\varepsilon^{012}=-1$. The 11-dimensional charge conjugation matrix $C$ is given by $C=1 \otimes \sigma_{2} \otimes \sigma_{1}$, $\bar{\psi}_{\alpha}=-\psi^{\beta} C_{\beta \alpha}\left(C_{\alpha \beta}=-C_{\beta \alpha}\right)$ and the super 3-form $B_{C B A}$ satisfies $d B=H$, with all components of $H$ vanishing except $H_{\mu \nu \alpha \beta}=-\frac{1}{3}\left(C \Gamma_{\mu \nu}\right)_{\alpha \beta}$. In addition to Poincaré invariance and world-sheet diffeomorphism invariance, the above action is also invariant under rigid space-time supersymmetry transformations and local Siegel transformations [183, 184].

Solving for $B_{C B A}$, the action may be rewritten in terms of $g^{i j}, \Pi_{i}^{\mu}$ and $\psi$, i.e.

$$
\begin{aligned}
I= & -\int d^{3} \xi\left[\sqrt{-g}\left(g^{i j} \Pi_{i}^{\mu} \Pi_{j \mu}-1\right)+\varepsilon^{i j k} \Pi_{i}^{\mu} \Pi_{j}^{\nu} \bar{\psi} \Gamma_{\mu \nu} \partial_{k} \psi\right. \\
& \left.-i \varepsilon^{i j k} \Pi_{i}^{\mu} \bar{\psi} \Gamma_{\mu \nu} \partial_{j} \psi \bar{\psi} \Gamma^{\nu} \partial_{k} \psi-\frac{1}{3} \varepsilon^{i j k} \bar{\psi} \Gamma_{\mu \nu} \partial_{i} \psi \bar{\psi} \Gamma^{\mu} \partial_{j} \psi \bar{\psi} \Gamma^{\nu} \partial_{k} \psi\right] .
\end{aligned}
$$

The corresponding equations of motion read

$$
\partial_{i}\left(\sqrt{-g} g^{i j} \Pi_{j}^{\mu}\right)+\varepsilon^{i j k} \Pi_{i}^{\nu} \partial_{j} \bar{\psi} \Gamma_{\nu}^{\mu} \partial_{k} \psi=0, \quad(1-\Gamma) g^{i j} \Pi_{i}^{\mu} \Gamma_{\mu} \partial_{j} \psi=0,
$$

in which $\Gamma=i \varepsilon^{i j k} \Pi_{i}^{\mu} \Pi_{j}^{\nu} \Pi_{k}^{\rho} \Gamma_{\mu \nu \rho} / 6 \sqrt{-g}$.

Now let us make some considerations for generic $p$. The variation of the Wess-Zumino-Witten term

$$
\varepsilon^{i_{1} \ldots i_{p+1}} \Pi_{i_{1}}^{A_{1}} \ldots \Pi_{i_{p+1}}^{A_{p+1}} B_{A_{p+1} \ldots A_{1}}
$$

of the global supersymmetric $p$-brane action is a total derivative if and only if the $\Gamma$-matrix identity holds for arbitrary spinors, namely

$$
\varepsilon^{i j k}\left[\Gamma^{\mu_{1}} \psi_{i}\left(\bar{\psi}_{j} \Gamma_{\mu_{1} \ldots \mu_{p}} \psi_{k}\right)+\Gamma_{\mu_{1} \ldots \mu_{p}} \psi_{i}\left(\bar{\psi}_{j} \Gamma^{\mu_{1}} \psi_{k}\right)\right]=0 .
$$

There exists only a finite number of admissible pairs $(p, D)$ for each spinor type. The $\Gamma$-matrix identity holds for arbitrary Majorana spinors only if $(p, D)$ is equal to one of the following pairs: $(1,3),(1,4),(2,4),(2,5),(2,7),(2,11),(3,8)$ or $(4,9)$. If the spinors are both Majorana and Weyl we also have $(p, D)=(1,10)$ and $(p, D)=(5,10)$. Finally for Weyl spinors we have $(p, D)=(1,4),(1,6),(2,4)$ and $(3,6)$. Note that for Dirac spinors the $\Gamma$-matrix identity is never satisfied. The Fermionic degrees of freedom of a $p$-brane are described by spinor of minimal size at each allowed $D$. When $p=1$, it follows the well known result that the classical Green-Schwarz superstring may be formulated only in $D=3,4,6,10$. Each of these string cases extends to other allowed $(p, D)$ pairs by simultaneously increasing $p$ and $D$ in the $(p, D)$ plane 


\begin{tabular}{|c|c|c|c|c|c|c|}
\hline Algebra & \multicolumn{5}{|c|}{$(p, D)$} & Codimension $D-p-1$ \\
\hline$R$ & $(1,3)$ & $(2,4)$ & & & & 1 \\
\hline$C$ & $(1,4)$ & $(2,5)$ & $(3,6)$ & & & 2 \\
\hline$H$ & $(1,6)$ & $(2,7)$ & $(3,8)$ & $(4,9)$ & $(5,10)$ & 4 \\
\hline$O$ & $(1,10)$ & $(2,11)$ & & & & 8 \\
\hline
\end{tabular}

Table 1:

up to $D=11$. The four discrete series are related to the four composition-division algebras $R, C, H$ and $O$ 185, 186, 171] as shown in Table 1. The case $p=0$ (superparticle) has been excluded. In this case, the Wess-Zumino-Witten term can be interpreted as a mass term [187]. Note that the codimension $(D-p-1)$ of the allowed $p$-branes equals the dimension of the related composition-division algebra. The $p$-branes in each series can be obtained from the highest- $p$ one (maximal superimmersion) by simultaneous dimensional reduction of the space-time and world-volume [148].

In order to obtain the simplest stable classical solution of the non-linear equations of motion (5.8), one can consider the supermembrane in a space-time with topology $S^{1} \times S^{1} \times \mathbb{R}^{9}$ [188. As a result the stabilization is carried out by the supermembrane stretching over the 2 -torus. The classical solution takes the form of a purely bosonic background with $\psi=0$ (for more details see Ref. [188]). Then one can quantize the linearized fluctuations around this background.

This can be generalized to a compactified (super) $p$-brane. Such a semiclassical quantization leads to the algebra of number operators $N_{\vec{n}}$ and (anti)commutation relations [188, 189, 190]. Finally, in Ref. [191] the critical dimension for the supermembrane is reported to be $D=11$, namely one of the classically admitted dimensions, which has been described above.

\subsection{Classification of 3-geometries}

The partition function as the fundamental object in the $p$-brane quantization may be expressed by means of a path integral evaluated over all the $p$-dimensional manifolds and the metrics on them. In particular, the functional integration over the 3-dimensional metric can be separated into an integration over all metrics for a 3 -volume of definite topology, followed by a sum over all topologies [192]. But even for a 3-dimensional manifold of fixed topology, the moduli space of all metrics, modulo 3-dimensional diffeomorphisms, is infinite dimensional. Here we shall present a necessarily brief description of the classification (uniformization) and sum over the topology for 3 and 4-dimensional manifolds.

The uniformization concept is one of the main concepts in complex analysis and other areas of mathematics. Here we shall discuss mainly uniformization of complex algebraic or more general analytic curves, i.e. Riemann surfaces and also multi-dimensional real manifolds admitting a conformal structure.

It should be recall that all curves of genus zero can be uniformized by rational functions, all those of genus one can be uniformized by elliptic functions, and all those of genus $g>1$, can be uniformized by meromorphic functions, defined on proper open subsets of $\mathscr{C}$, for example in the disk. This result, due to Klein, Poincaré and Koebe, is one of the deepest achievements in mathematics as a whole. A complete solution of the uniformization problem has not yet been obtained (with the exception of the 1-dimensional complex case). However, there have been essential advances in this problem, which have brought to foundations for topological methods, covering spaces, existence theorems for partial differential equations, existence and distorsion theorems for conformal mappings and so on. 
With regard to one-dimensional complex manifolds, in accordance with Klein-Poincaré uniformization theorem, each Riemann surface can be represented (within a conformal equivalence) in the form $\Sigma / \Gamma$, where $\Sigma$ is one of the three canonical regions, namely the extended plane $\overline{\mathcal{C}}$ (the sphere $\left.S^{2}\right)$, the plane $\mathbb{C}\left(\mathbb{R}^{2}\right)$, or the disk, and $\Gamma$ is a discrete group of Möbius automorphisms of $\Sigma$ acting freely there (without fixed points). Riemann surfaces with such coverings are elliptic, parabolic and hyperbolic type respectively. The theorem given above admits generalization also to surfaces with branching.

A different approach to the solution of the uniformization problem was proposed by Koebe. The general uniformization principle of Koebe asserts that if a Riemann surface $\tilde{\Sigma}$ is topologically equivalent to a planar region $P$, then there also exists a conformal homeomorphism of $\tilde{\Sigma}$ onto $P$. The same problem of analytic uniformization reduces to the topological problem of finding all the (branched, in general) planar coverings $\tilde{\Sigma} \mapsto \Sigma$ of a given Riemann surface $\Sigma$. The solution of this topological problem is given by the theorem of Maskit.

It should be noted that, with the help of standard uniformization theorems and decomposition theorems [193], one can construct and describe all the uniformizations of Riemann surfaces by Kleinian groups. Furthermore, by using the quasiconformal mappings, it is possible to obtain an uniformization theorem of more general character (this fact is related to Techmüller spaces), namely it is possible to prove that several surfaces can be uniformized simultaneously (see for example Ref. [194]).

The Thurston classification In the path integral approach to membranes the two following problems arise. Should one include all 3-dimensional manifolds or only orientable ones? What is known about the classification of manifolds?

From the physical point of view, we shall restrict ourselves only to orientable 3-dimensional manifolds (see for example [192]). Furthermore, in dealing with the evaluation of the vacuum persistence amplitude, we shall consider the sum over all compact orientable manifolds without boundaries. With regard to the classification of the 3-dimensional manifolds, this is a difficult problem but important progress has been made by Thurston [195].

It is well known that for any closed orientable 2-dimensional manifold $\mathcal{M}$ the following result holds: every conformal structure on $\mathcal{M}$ is represented by a constant curvature geometry. The only simply connected manifolds with constant curvature are $\Sigma=S^{2}$ or $\mathbb{R}^{2}$ or $H^{2}$ and $\mathcal{M}$ can be represented as $\Sigma / \Gamma$, where $\Gamma$ is a group of isometries.

Let us now turn to the classification of the 3-geometries following the presentation of Ref. [196]. By a geometry or a geometric structure we mean a pair $(\Sigma, \Gamma)$, that is a manifold $\Sigma$ and a group $\Gamma$ acting transitively on $\Sigma$ with compact point stabilizers. Two geometries $(\Sigma, \Gamma)$ and $\left(\Sigma^{\prime}, \Gamma^{\prime}\right)$ are equivalent if there is a diffeomorphism of $\Sigma$ with $\Sigma^{\prime}$ which throws the action of $\Gamma$ onto the action of $\Gamma^{\prime}$. In particular, $\Gamma$ and $\Gamma^{\prime}$ must be isomorphic. We shall assume:

i) The manifold $\Sigma$ is simply connected. Otherwise it will be sufficient to consider a natural geometry $(\tilde{\Sigma}, \tilde{\Gamma}), \tilde{\Sigma}$ being the universal covering of $\Sigma$ and $\tilde{\Gamma}$ denoting the group of all diffeomorphisms of $\tilde{\Sigma}$ which are lifts of elements of $\Gamma$.

ii) The geometry admits a compact quotient. In another words, there exists a subgroup $G$ of $\Gamma$ which acts on $\Sigma$ as covering group and has compact quotient.

iii) The group $\Gamma$ is maximal. Otherwise, if $\Gamma \subset \Gamma^{\prime}$ then any geometry $(\Sigma, \Gamma)$ would be the geometry $\left(\Sigma, \Gamma^{\prime}\right)$ at the same time.

After these preliminaries we can state the classification theorem.

Theorem 3 (Thurston) Any maximal, simply connected, 3-dimensional geometry admitting a compact quotient is equivalent to one of the geometries $(\Sigma, \Gamma)$, where $\Sigma$ is one of the eight manifolds $\mathbb{R}^{3}, S^{3}, H^{3}, S^{2} \times \mathbb{R}, H^{2} \times \mathbb{R}, S \widetilde{L(2, \mathbb{R}), N i l}$, Sol. 
The group properties and more details of these manifolds may be found in Ref. [196]. The first five geometries are familiar objects, so we explain metric and isometry group of the last three ones.

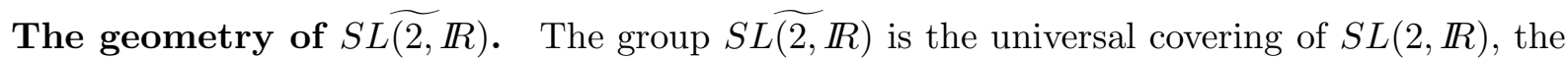
3 -dimensional Lie group of all $2 \times 2$ real matrices with determinant 1 . The standard metric on $S L \widetilde{(2, \mathbb{R})}$ is one of the left (right)-invariant metrics. It is well known that for a Riemannian $\mathrm{N}$-dimensional manifold $\mathcal{M}$ there is a natural $2 \mathrm{~N}$-dimensional metric on the tangent bundle $T \mathcal{M}$ of $\mathcal{M}$. If $f: \mathcal{M} \rightarrow \mathcal{M}$ is an isometry, then $d f: T \mathcal{M} \rightarrow \mathcal{T} \mathcal{M}$ is also an isometry. We shall use this argument for the hyperbolic plane $\mathcal{M}=\mathcal{H}^{\epsilon}$. The unit tangent bundle $U H^{2}$ of $H^{2}$ has a metric induced from the base manifold $T H^{2}$. Since there is a natural identification of $U H^{2}$ with $P S L(2, \mathbb{R})$, the orientation preserving isometry group of $H^{2}$, then we have a metric on $\operatorname{PSL}(2, \mathbb{R})$. Note that $P S L(2, \mathbb{R})$ is doubly covered by $S L(2, \mathbb{R})$, therefore its universal covering is $S L \widetilde{(2, \mathbb{R})}$ and the induced metric on $S L \widetilde{(2, \mathbb{R})}$ is the one in which we are interested. Finally $S L \widetilde{(2, \mathbb{R})}$ is naturally a line bundle over $H^{2}$ since the bundle $U H^{2}$ is a circle bundle over $H^{2}$. The 4-dimensional isometry group of $S L \widetilde{(2, \mathbb{R})}$ preserves this bundle structure and has two components both orientation preserving [196].

The geometry of Nil. Nil is the 3-dimensional Lie group of all $3 \times 3$ real upper triangular matrices of the form

$$
\left(\begin{array}{lll}
1 & x & z \\
0 & 1 & y \\
0 & 0 & 1
\end{array}\right)
$$

with ordinary matrix multiplication, $x, y$ and $z$ being real numbers. It is also known as the nilpotent Heisenberg group. It is easy to write down a metric which is invariant under left multiplication for Nil. A basis of left-invariant 1-forms is

$$
\sigma^{1}=d z-x d y, \quad \sigma^{2}=d x, \quad \sigma^{3}=d y .
$$

Therefore, the standard metrics reads

$$
d s^{2}=\left(\sigma^{1}\right)^{2}+\left(\sigma^{2}\right)^{2}+\left(\sigma^{3}\right)^{2}=d x^{2}+d y^{2}+(d z-x d y)^{2} .
$$

The isometry group has Nil as its subgroup. There is an additional one-parameter family of isometries isomorphic to $U(1)$ which can be written as $(0 \leq \theta<2 \pi)$

$$
S_{\theta}:\left(\begin{array}{l}
x \\
y \\
z
\end{array}\right) \rightarrow\left(\begin{array}{c}
x \cos \theta+y \sin \theta \\
-x \sin \theta+y \cos \theta \\
z+\frac{1}{2}\left[\left(x^{2}-y^{2}\right) \cos \theta-2 x y \sin \theta\right] \sin \theta
\end{array}\right) .
$$

Here the 2-dimensional rotation matrix of angle $\theta$ appears. The 4-dimensional isometry group has two components. A discrete isometry is given by $(x, y, z) \rightarrow(x,-y,-z)$ and besides, all isometries preserve the orientation of $\mathrm{Nil}$.

The geometry of Sol. Sol is the 3-dimensional (solvable) group with the following multiplication rule

$$
\left(\begin{array}{l}
x \\
y \\
z
\end{array}\right)\left(\begin{array}{l}
x^{\prime} \\
y^{\prime} \\
z^{\prime}
\end{array}\right)=\left(\begin{array}{c}
x+e^{-z} x^{\prime} \\
y+e^{z} y^{\prime} \\
z+z^{\prime}
\end{array}\right)
$$


A basis of left-invariant 1 - forms is

$$
\sigma^{1}=e^{z} d x, \quad \sigma^{2}=e^{-z} d y, \quad \sigma^{3}=d z,
$$

while the standard left-invariant metric reads

$$
d s^{2}=\left(\sigma^{1}\right)^{2}+\left(\sigma^{2}\right)^{2}+\left(\sigma^{3}\right)^{2}=e^{2 z} d x^{2}+e^{-2 z} d y^{2}+d z^{2} .
$$

The discrete isometries are

$$
(x, y, z) \rightarrow\left\{\begin{array}{c}
( \pm x, \pm y, z) \\
( \pm y, \pm x,-z)
\end{array}\right.
$$

so the group $\Gamma$ of $S o l$ has eight components. Moreover, four of them, connected to the following elements, are orientation preserving:

$$
(x, y, z) \rightarrow\left\{\begin{array}{c}
(x, y, z) \\
(-x,-y, z) \\
(y, x,-z) \\
(-y,-x,-z)
\end{array}\right.
$$

As for the manifolds modelled on $H^{2} \times \mathbb{R}, S^{1} \times H^{2} / \Gamma$ contains a compact Riemann surface and

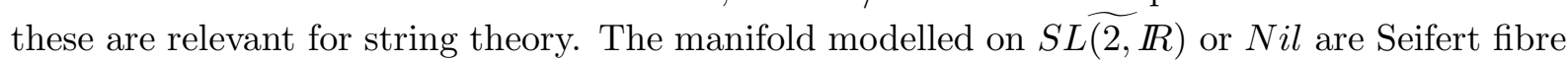
spaces and those modelled on $S o l$ are bundles over $S^{1}$ with fibers the torus or the Klein bottle. A compact 3-manifold without boundary modelled on $\mathbb{R}^{3}, S^{3}, S^{2} \times \mathbb{R}, H^{2} \times \mathbb{R}, S \widetilde{(2, \mathbb{R}), N i l}$ is a Seifert fibre space and vice-versa [196].

\subsection{Classification of 4-geometries}

Unlike the case of compact Riemann surfaces or 3-dimensional manifolds, very little is known about the uniformization of $N$-dimensional manifolds $(N>3)$ by Kleinian groups. The reader can find some results along these lines for conformal manifolds in Ref. [197].

The Donaldson theorem [198] for smooth structure on $\mathbb{R}^{4}$ shows that in the theory of differentiable 4-dimensional manifolds is necessary to use low dimensional methods, in particular geometrical methods. Although in this case there are no decomposition theorems which permit the use of Thurston methods, nevertheless there is a classification of the 4-dimensional geometries $(\Sigma, \Gamma)$ 199]. The reader can find some necessary informations about 4-geometries from the point of view of homogeneous Riemannian manifolds and Lie groups in Refs. [200, 201].

The list of Thurston 3-geometries can be organized in terms of the compact stabilizers $\Gamma_{\sigma}$ of $\sigma \in \Sigma$ isomorphic to $S O(3), S O(2)$ or trivial group $S O(1)$. The analogue list of 4-geometries can be organized (using only connected groups of isometries) as in in Table 2. Here we have the four irreducible 4-dimensional Riemannian symmetric spaces: sphere $S^{4}$, hyperbolic space $H^{4}$, complex projective space $\mathbb{C} P^{2}$ and complex hyperbolic space $\mathbb{C} H^{2}$ (which we may identify with the open unit ball in $\mathbb{C}^{2}$ with an appropriate metric). The other cases are more specific and for the sake of completeness we shall illustrate them.

The geometry of $N i l^{4}, S_{m, n}^{4}, S_{0 l}^{4}$ and $F^{4}$. The nilpotent Lie group $N i l^{4}$ can be presented as the split extension $\mathbb{R}^{3} \oslash_{U} \mathbb{R}$ of $\mathbb{R}^{3}$ by $\mathbb{R}$ (the symbol $\oslash$ denotes semidirect product). The quotient $\mathbb{R}$ acts on the subgroup $\mathbb{R}^{3}$ by means of $U(t)=\exp (t B)$, where

$$
B=\left(\begin{array}{lll}
0 & 1 & 0 \\
0 & 0 & 1 \\
0 & 0 & 0
\end{array}\right) .
$$




\begin{tabular}{||l|l||}
\hline stabilizer $\Gamma_{\sigma}$ & manifold $\Sigma$ \\
\hline$S O(4)$ & $S^{4}, \mathbb{R}^{4}, H^{4}$ \\
$U(2)$ & $\mathbb{C} P^{2}, \mathbb{C} H^{2}$ \\
$S O(2) \times S O(2)$ & $S^{2} \times \mathbb{R}^{2}, S^{2} \times S^{2}, S^{2} \times H^{2}, H^{2} \times \mathbb{R}^{2}, H^{2} \times H^{2}$ \\
$S O(3)$ & $S^{3} \times \mathbb{R}, H^{3} \times \mathbb{R}$ \\
$S O(2)$ & $\left.N i l^{3} \times R, P S \widetilde{L(2,}, \mathbb{R}\right) \times \mathbb{R}, S o l^{4}$ \\
$S^{1}$ & $F^{4}$ \\
trivial & $N i l^{4}, S o l_{m, n}^{4}\left(\right.$ including $\left.S o l^{3} \times \mathbb{R}\right), S o l_{1}^{4}$ \\
\hline
\end{tabular}

Table 2:

In the same way, for the soluble Lie groups one has $S o l_{m, n}^{4}=\mathbb{R}^{3} \oslash_{T_{m, n}} \mathbb{R}$, where $T_{m, n}(t)=$ $\exp \left(t C_{m, n}\right)$ and

$$
C_{m, n}=\left(\begin{array}{ccc}
\alpha & 0 & 0 \\
0 & \beta & 0 \\
0 & 0 & \gamma
\end{array}\right),
$$

with the real numbers $\alpha>\beta>\gamma$ and $\alpha+\beta+\gamma=0$. Furthermore $e^{\alpha}, e^{\beta}$ and $e^{\gamma}$ are the roots of $\lambda^{3}-m \lambda^{2}+n \lambda-1=0$, with $m, n$ positive integers. If $m=n$, then $\beta=0$ and $S_{o l} l_{m, n}^{4}=S o l^{3} \times \mathbb{R}$. In general, if $C_{m, n} \propto C_{m^{\prime}, n^{\prime}}$, then $S_{\text {ol }} l_{m, n}^{4} \cong S_{\text {ol }} m_{m^{\prime}, n^{\prime}}^{4}$. When $m^{2} n^{2}+18=4\left(m^{3}+n^{3}\right)+27$, one has a new geometry, $\mathrm{Sol}_{0}^{4}$, associated with group $S O(2)$ of isometries rotating the first two coordinates.

The soluble group $S o l_{1}^{4}$, is most conveniently represented as the matrix group

$$
\left(\begin{array}{ccc}
1 & b & c \\
0 & \alpha & a \\
0 & 0 & 1
\end{array}\right),
$$

with $\alpha, a, b, c \in \mathbb{R}, \alpha>0$.

Finally the geometry $F^{4}$, related to the isometry group $\mathbb{R}^{2} \oslash P S L(2, \mathbb{R})$ and stabilizer $S O(2)$, is the only geometry which admits no compact model. A connection of these geometries with complex and Kählerian structures (preserved by the stabilizer $\Gamma_{\sigma}$ ) can be found in Ref. 199]

We conclude this subsection with some remarks. It is well known that there are only a finite number of manifolds of the form $\mathbb{R}^{N} / \Gamma, S^{N} / \Gamma$ for any $N$ [103]. A fortiori this holds also for $S^{2} \times \mathbb{R}^{2}, S^{2} \times S^{2}, S^{3} \times \mathbb{R}$ manifolds. Besides, if we make the intuitive requirement that only irreducible manifolds have to be taken into account, then the manifolds modelled on $S^{2} \times \mathbb{R}$, $H^{2} \times \mathbb{R}$ have to be excluded in 3-dimensions, while the ones modelled on

$$
\begin{aligned}
& S^{2} \times \mathbb{R}^{2}, S^{2} \times S^{2}, S^{2} \times H^{2}, H^{2} \times \mathbb{R}^{2}, H^{2} \times H^{2}, \\
& \left.S^{3} \times \mathbb{R}, H^{3} \times \mathbb{R}, N i l^{3} \times \mathbb{R}, P S \widetilde{L(2}, \mathbb{R}\right) \times \mathbb{R}, S o l^{3} \times \mathbb{R}
\end{aligned}
$$

have to be neglected in 4-dimensions. As a consequence it seems that the more important contribution to the vacuum persistence amplitude should be given by the compact hyperbolic geometry, the other geometries appearing only for a small number of exceptions [201]. It has to be noted that gluing of the above geometries, characterizing different coupling constants, by a complicated set of moduli, is a very difficult task (for more details see Refs. [202, 203]). Therefore, in the following physical applications, we shall consider the compact hyperbolic manifolds $H^{N} / \Gamma$. 


\subsection{The path integral associated with loop expansion}

In this subsection a path integral technique for the closed quantum $p$-brane will be considered. Such an approach has been pioneered for the string case $(p=1)$ in Ref. [180]. More recently (see Refs. [121, 204, 205]) it has been used to discuss the quantization of a closed $p$-brane when the extended object sweeps out a compact $(p+1)$-dimensional manifold without boundary.

In the framework of the path integral approach, the idea is to find a classical solution of the equation of motion, expand the action up to quadratic terms in fluctuations around the classical solution and compute the determinants of second order elliptic operators which arise in the Gaussian functional integration. Such operators are always Laplace-Beltrami type operators, acting in different bundles over the above compact manifold. They can be considered as the main building blocks of the $p$-brane path integral.

Note that the usual perturbative expansion methods cannot be applied. It would be nice to quantize covariantly the model, but from the experience with the string case, it is known that this may be extremely difficult. It is also less satisfactory to fix the reparametrization invariance in a particular gauge, checking the covariance afterwards. As far as this issue is concerned, it is known [206] that for $p>1$ there exists no gauge in which the model can be cast in a linear form. As a consequence, as a first step to quantization of a non-linear theory, one can attempt a semiclassical (one-loop) approximation [207.

\subsubsection{The free relativistic point particle}

With regard to the path integral quantization, let us show how action defined by Eq. (5.4) leads quite naturally to the point particle Euclidean relativistic propagator. Indeed it is well known that the propagator related to the relativistic point particle can be written as

$$
A\left(X, f ; X^{\prime}, f^{\prime}\right)=N \int d[X] d[f] \exp \left[-\int_{0}^{1}\left(\frac{g_{\mu \nu} \dot{X}^{\mu} \dot{X}^{\nu}}{2 \dot{f}}+\frac{m^{2} \dot{f}}{2}\right) d \xi\right] .
$$

We may choose $f(0)=0$ and $f(1)=c>0$, the meaning of $c$ being the length of the trajectory. Note that

$$
c=\int_{0}^{1} \frac{d f}{d \xi} d \xi, \quad \int_{0}^{1} \frac{g_{\mu \nu} \dot{X}^{\mu} \dot{X}^{\nu}}{\dot{f}} d \xi=\int_{0}^{c} g_{\mu \nu} \frac{d X^{\mu}}{d f} \frac{d X^{\nu}}{d f} d f .
$$

Let us introduce $f=c \rho$ so that $d[f]=d c d[\rho]$. Thus, we arrive at

$$
A\left(X, f ; X^{\prime}, f^{\prime}\right)=N \int d[\rho] \int_{0}^{\infty}\left[e^{-\frac{m^{2} c}{2}} \int d[X] \exp \left(-\int_{0}^{c} g_{\mu \nu} \frac{d X^{\mu}}{d f} \frac{d X^{\nu}}{d f} d f\right)\right] d c
$$

The true propagator can be obtained factorizing out the infinite measure due to reparametrization invariance of the action. Thus, we get the well known result

$$
A\left(X ; X^{\prime}\right)=\frac{1}{(4 \pi)^{D / 2}} \int_{0}^{\infty} c^{-\frac{D}{2}} e^{-\frac{m^{2} c}{2}-\frac{\left(X-X^{\prime}\right)^{2}}{4 c}} d c .
$$

\subsubsection{The $p$-brane model}

Here we shall mimic the approach used in the point-like case. To start with, the partition function which describes the quantized extended object may be written as

$$
Z=N \int d[f] d[X] \exp \left[-\int\left(\frac{\gamma}{2 F}+\frac{k^{2} F}{2}\right) d^{p+1} \xi\right] .
$$


An argument similar to the one given above for the point particle leads to

$$
Z=N \int d[\rho] \int_{0}^{\infty}\left[\exp \left(-\frac{k^{2} \Omega}{2}\right) \int d[X] \exp \left(-\int \gamma d^{p+1} f\right)\right] d \Omega,
$$

where $\Omega$ is the volume of the $(p+1)$-dimensional closed manifold and the infinite integration associated with reparametrization invariance of the model has been factorized out. This approach to quantization of extended objects has been proposed for strings in Ref. 180 (see also Ref. [208]). The evaluation of the above functional integral is a formidable problem. For $p>2$, one is forced to make use of the Gaussian approximation. With regard to this, we would like to recall that the main issue one has to deal with, is the classification of $(p+1)$-dimensional closed manifolds and the related evaluation of determinant of Laplacian operators. To our knowledge, such a task is far from being solved. We have argued previously that the compact hyperbolic geometries seem to play a significant role among all the possible ones. As a consequence, it seems reasonable to consider within the semiclassical approximation, that the bosonic contribution of the $p$-brane is represented by factors like $(\operatorname{det} L)^{-(D-p-1) / 2}, L$ being a suitable Laplace-Beltrami operator acting over $\mathcal{M}^{\mathcal{N}}=\mathcal{H}^{\mathcal{N}} /$ - Furthermore one should observe that in general, there exist a number of different topologically inequivalent real bundles over $\mathcal{M}^{\mathcal{N}}$, this number being given by the number of elements of $H^{1}\left(\mathcal{M}^{\mathcal{N}} ; \mathbb{Z}_{\in}\right)$, the first cohomology group of $\mathcal{M}^{\mathcal{N}}$ with coefficients in $\mathbb{Z}_{2}$. Thus, one has to try to evaluate such determinants on compact manifolds, the hyperbolic ones, i.e. $\mathcal{M}^{\mathcal{N}}=\mathcal{H}^{\mathcal{N}} /$ - giving the most important contributions.

At the end of Sec. 3.4.6 we have evaluated the regularized determinant on hyperbolic manifolds by taking into account also the possible presence of zero modes, which strictly depends on the characters $\chi$. We have derived the equation (see Eq. (3.60))

$$
\operatorname{det} L_{N}=\frac{1}{\mathcal{N} !} \exp \left[-\gamma K_{N}\left(L_{N}\right)-I\left(0 \mid L_{N}\right)\right] Z_{N}^{(\mathcal{N})}\left(2 \varrho_{N}\right),
$$

where $L_{N}=-\Delta_{N}$ and $\mathcal{N}$ is the number of zero-modes. For example, for $N=2$ and trivial real line bundle $(\chi=1), s=1$ is a zero with multiplicity 1 of $Z_{2}(s)$. In this case (string model), a formula similar to Eq. (5.14) has been discussed in Refs. 209, 210, 93].

For $N=3$ (membrane model), the situation is quite similar and the evaluation of the Laplace determinant has been done in Ref. [121], but with a different technique. Also in this case, for untwisted fields $(\chi=1)$ there exists a zero mode. Moreover, $K_{3}\left(L_{3}\right)=0$ (odd dimension) and from Eq. (3.55), $I\left(0 \mid L_{3}\right)=\Omega\left(\mathcal{F}_{3}\right) / 6 \pi$ easily follows. As a consequence we obtain the simple result for the determinant of the Laplacian

$$
\operatorname{det} L_{N}=Z_{3}^{\prime}(2) \exp \left[-\frac{\left.\Omega_{(} \mathcal{F}_{3}\right)}{6 \pi}\right] \text {. }
$$

As a result, it follows that the leading contribution seems to come from 3-dimensional compact hyperbolic manifolds having the smallest volume.

In this Section, we have discussed in some detail hyperbolic contribution to the one-loop approximation of closed $p$-branes. It has also been proposed a slight variation of Dirac $p$-brane action which however involve a set of new scalar fields necessary for the reparametrization invariance of the action. Even though at the classical level this action leads to some simplification, it is not clear if, at the quantum level, such simplifications still remain. However, within the one-loop approximation the bosonic sectors are all equivalent. The main issue to be solved is the evaluation of a Laplace determinant for scalar fields on a compact $(p+1)$-dimensional manifold swepts out by the $p$-brane.

As far as the extension of these results to super $p$-branes is concerned, we note that at the classical level the fermionic sector may present some difficulties which can be overcome (see Ref. [153]). At the quantum level, one should deal with determinants of the square of the Dirac 
operator on $(p+1)$-dimensional compact hyperbolic manifolds. To our knowledge only the string case has been successfully considered (see Ref. [209, 210]).

\subsection{The Casimir energy for $p$-branes in space-times with constant curvature}

The physical properties of a $p$-brane in the quantum regime may be obtained from a study of the effective action for various $p$-brane configurations. The first attempts along these lines have been performed for bosonic and supersymmetric membranes in Ref. 149, 150, 151, for bosonic membrane in a 1/D approximation in Refs. [211, 212, 213] and for bosonic $p$-branes in Ref. 157]. Open, toroidal and spherical $p$-branes have been considered [214] with the interesting result that the Casimir energy provides a repulsive force which stabilizes the membrane at non zero radius, but the net energy of this stabilized membrane is negative, suggesting that the membrane ground state is tachyonic. Since the Casimir energy is likely to vanish for the supermembrane, it seems unlikely that these results will carry over to that case.

Here we will present a general expression for the static potential (Casimir energy) of $p$-branes compactified on constant curvature Kaluza-Klein space-times [189, 215]. Thus we shall consider $p$-branes which evolve in space-times of the kind $\mathcal{M}=\mathcal{M}^{\mathcal{D}}=\mathcal{M}^{\sqrt{ }} \times \mathbb{R}^{\mathcal{D}-} \sqrt{ }(D>p)$ with $\mathcal{M}^{\vee}=\mathcal{T}^{\vee}, \mathcal{M}^{\vee}=\mathcal{T}^{\mathcal{K}} \times \mathcal{S}^{\mathcal{Q}}(p=K+Q), \mathcal{M}^{\vee}=\mathcal{T}^{\mathcal{K}} \times \mathcal{S}^{\mathcal{Q}} \times \mathcal{H}^{\mathcal{N}} /-(p=K+Q+N)$, $\Gamma$ being a discrete group of isometries of $H^{N}$.

Classical solutions and gauge conditions. In the case of toroidal $p$-brane configurations, i.e. $\mathcal{M} \sqrt{ }=\mathcal{T} \sqrt{ }$, we will consider the following classical solutions of the equation of motion

$$
\begin{array}{rlrl}
\vec{X}_{c l}^{0} \equiv \tau & =\xi_{0}, & \vec{X}_{c l}^{\perp}=0, & \vec{X}_{c l}^{D-1} \equiv \theta_{1}=\xi_{1}, \ldots, X_{c l}^{D-p}=\xi_{p}, \\
\left(\gamma_{c l}\right)_{i j} & =\eta_{i j}, &
\end{array}
$$

where $\vec{X}_{c l}^{\perp}=\left(X^{1}, \ldots, X^{D-p-1}\right)$ and $\left(\xi_{1}, \ldots, \xi_{p}\right) \in U=\left[O, r_{1}\right] \times \ldots \times\left[O, r_{p}\right], r_{i}$ being the circle radii of the space $T^{p}$. In all cases examined below, the fields are taken to be periodic in the imaginary time with period $T$, that is

$$
\vec{X}^{\perp}\left(0, \xi_{1}, \ldots, \xi_{p}\right)=\vec{X}^{\perp}\left(T, \xi_{1}, \ldots, \xi_{p}\right) .
$$

The nontrivial topology of a space-time leads to the existence of the topologically inequivalent field configurations of fields [125, 216]. The number of such configurations is equal to the number of non-isomorphic linear real vector bundles over $\mathcal{M}$, i.e. the number of elements in $H^{1}\left(\mathcal{M} ; \mathbb{Z}_{\in}\right)$, the first cohomology group of $\mathcal{M}$ with coefficients in $\mathbb{Z}_{2}$ [125, 216, 217, 218]. Each field sector is characterized by some quantum number, the Möbius character or twist $h \in H^{1}\left(\mathcal{M} ; \mathbb{Z}_{\in}\right)$. Since $H^{1}\left(\mathcal{M} ; \mathbb{Z}_{\in}\right)$ is always an abelian group, there exists the obvious condition $h^{2}=h+h=0 \in H^{1}\left(\mathcal{M} ; \mathbb{Z}_{\epsilon}\right)$. We introduce the vector $\vec{g}=\left(g_{1}, \ldots, g_{p}\right)$ which defines the type of field (i.e. the corresponding twist h). Depending on the field type chosen in $\mathcal{M}$, we have $g_{i}=0$ (untwisted field) or $1 / 2$ (twisted field). In our case $H^{1}\left(T^{p} ; \mathbb{Z}_{2}\right)=\mathbb{Z}_{2}^{p}$ and the number of configurations of real scalar field is $2^{p}$. Therefore we take as remaining boundary conditions, the $p$ equations

$$
\vec{X}^{\perp}\left(\xi_{0}, \xi_{1}, \ldots, \xi_{i}=0, \ldots, \xi_{p}\right)=\left(1-4 g_{i}\right) \vec{X}^{\perp}\left(\xi_{0}, \xi_{1}, \ldots, R_{T i}, \ldots, \xi_{p}\right),
$$

where the index $i$ runs from 1 to $p$. Eqs. (5.16) generalize the corresponding boundary conditions for the toroidal $p$-brane [157] (see also Refs. [212, 213]).

In the case of space-times with topology $T^{K} \times S^{Q}$ the only non-zero elements of the metric are given by

$$
\begin{aligned}
& g_{D-1, D-1}=R_{S}^{2}, \quad g_{D-j, D-j}=R_{S}^{2} \prod_{l=1}^{j-1} \sin ^{2}\left(\theta_{l}\right), \quad j=2, \ldots, Q, \\
& g_{i i}=1, \quad i=0,1, \ldots, D-(Q+1),
\end{aligned}
$$


where $R_{S}$ is the fixed radius of the hypersphere. We shall generalize the spherical $p$-brane results of Refs. [212, 213, 157] and we shall find the classical solution in the form

$$
\begin{aligned}
& \vec{X}_{c l}^{0}=\xi_{0}, \quad \vec{X}_{c l}^{\perp}=0, \quad X_{c l}^{D-1}=\xi_{1}, \ldots, X_{c l}^{D-p}=\xi_{p}, \\
& \left(\gamma_{c l}\right)_{i j} d \xi^{i} d \xi^{j}=d \tau^{2}+R_{S}^{2} d \Omega_{Q}^{2}+\sum_{l=Q+1}^{K} d \xi^{l} d \xi^{l} .
\end{aligned}
$$

There are two types of boundary conditions for the function $\vec{X}^{\perp}\left(\xi_{0}, \xi_{1}, \ldots, \xi_{K}, \ldots, \xi_{p}\right)$. The first of them (with respect to space-time parameters $\xi_{i}, i=Q+1, \ldots, K$ ) looks like Eqs. (5.16), while the second one (related to $\xi_{i}, i=1, \ldots, Q$ ) is the appropriate boundary conditions for a hypersphere.

Finally in the case $T^{K} \times S^{Q} \times\left(H^{N} / \Gamma\right)$ the nonzero elements of the metric are given by

$$
\begin{array}{rlrl}
g_{D-1, D-1} & =R_{S}^{2}, & g_{D-j, D-j}=R_{S}^{2} \prod_{l=1}^{j} \sin ^{2}\left(\theta_{l}\right), & j=2, \ldots, Q, \\
g_{D-i, D-i} & =R_{H}^{-2} \xi_{2}^{-2}, & i=Q+1, \ldots, Q+1+N, & \\
g_{l, l} & =1, & l=0,1, \ldots, D-(Q+1+N),
\end{array}
$$

where $-R_{H}^{-2}$ is the curvature of hyperbolic metric. The classical solution of Eqs. (5.17) has the form

$$
\begin{aligned}
& \vec{X}_{c l}^{0} \equiv \tau=\xi_{0}, \quad \vec{X}_{c l}^{\perp}=0, \quad X_{c l}^{D-1}=\xi_{1}, \ldots, X_{c l}^{D-p}=\xi_{p}, \\
& \left(\gamma_{c l}\right)_{i j} d \xi^{i} d \xi^{j}=d \tau^{2}+R_{S}^{2} d \omega_{Q}^{2}+\xi_{2}^{-2} R_{H}^{-2} d \Omega_{N}^{2}+\sum_{l=K+1}^{Q+N} d \xi^{l} d \xi^{l} .
\end{aligned}
$$

Let us make some considerations necessary for further calculations. Since the fundamental group of the manifold $\mathcal{M}$ now is $\mathbb{Z}^{K} \times \Gamma$, it follows that the real bundles over $\mathcal{M}$ correspond to multiplets $(\vec{g}, \vec{\chi})$. Here $\vec{\chi}$ is a character of the group $\Gamma$. For example a scalar Laplacian $L_{D}$ in such a bundle is the Kronecker sum of the following Laplacians: a Laplacian $L_{K}$ in the real line bundle labelled by $\vec{g}$ over torus $T^{K}$, the standard Laplacian $L_{Q}$ on the $Q$-dimensional sphere and a Laplacian $L_{N}$ in the real line bundles $\vec{\chi}$ over $H^{N} / \Gamma$. Therefore we have three types of boundary conditions for the functions $\vec{X}^{\perp}\left(\xi_{0}, \ldots, \xi_{p}\right)$. The first of them is the boundary conditions for torus (it looks like Eq. (5.16)). The second and the third types, concerning the parameters $\xi_{i}$ for $i=1, \ldots, Q$ and $i=Q+1, \ldots, Q+N$, are the appropriate boundary conditions for hyperspheres and compact hyperbolic spaces correspondingly. Note that in all cases examined below we shall use the background gauge as in Refs. [211, 212, 213, 157, 215]

$$
X^{0}=X_{c l}^{0}, X^{D-1}=X_{c l}^{D-1}, \ldots, X^{D-p}=X_{c l}^{D-p},
$$

in which there are no Faddeev-Popov ghosts.

\subsubsection{The semiclassical approximation}

Here we shall derive the expression of the static potential keeping only the quadratic quantum fluctuations around a static classical solution and making use of the background field gauge. It is easy to show that in this approximation all the actions we have considered reduce to

$$
S=k T \Omega_{p}+\frac{1}{2} \int_{0}^{T} d \xi_{0} \int_{\mathcal{M}^{p}} \vec{\eta}^{\perp} \cdot L \vec{\eta}^{\perp} d \xi
$$


where $L$ is the Laplace operator acting on the transverse fluctuation fields $\vec{\eta}^{\perp}$. The Euclidean vacuum-vacuum amplitude reads

$$
\begin{aligned}
Z & =\int d\left[\vec{\eta}^{\perp}\right] e^{-S}=e^{-k T \Omega_{p}} \int d\left[\vec{\eta}^{\perp}\right] \exp \left(-\frac{1}{2} \int_{0}^{T} d \xi_{0} \int_{\mathcal{M}^{p}} \vec{\eta}^{\perp} \cdot k L \vec{\eta}^{\perp} d \xi\right) \\
& =e^{-k T \Omega_{p}}\left(\operatorname{det} k L \ell^{2}\right)^{-\frac{D-p-1}{2}} .
\end{aligned}
$$

So one has

$$
\ln Z=-k T \Omega_{p}+\frac{D-p-1}{2} \zeta^{\prime}\left(0 \mid k L \ell^{2}\right) .
$$

The static potential is defined by

$$
V=-\lim _{T \rightarrow \infty} \frac{\ln Z}{T}=k \Omega_{p}-\frac{D-p-1}{2} \lim _{T \rightarrow \infty} \frac{\zeta^{\prime}\left(0 \mid k L \ell^{2}\right)}{T} .
$$

A direct computation gives (see Eq. (2.35) with $p=1$ and $N=p$ )

$$
\zeta(s \mid L)=\frac{T \Gamma\left(s-\frac{1}{2}\right)}{\sqrt{4 \pi} \Gamma(s)} \zeta\left(s-\frac{1}{2} \mid L_{p}\right)+O\left(e^{-T^{2}}\right),
$$

in which $L_{p}$ is the Laplace operator on $\mathcal{M}^{\vee}$. Using Eq. (2.36) we can immediately write down

$$
\begin{aligned}
V & =k \Omega_{p}+\frac{D-p-1}{2} \zeta^{(r)}\left(-\frac{1}{2} \mid k L_{p} \ell^{2}\right) \\
& =k \Omega_{p}+\frac{D-p-1}{2}\left[\frac{\ln \left(4 k \ell^{2}\right)-2}{\sqrt{4 \pi}} K_{p+1}\left(L_{p}\right)+\operatorname{PP} \zeta\left(-\frac{1}{2} \mid L_{p}\right)\right],
\end{aligned}
$$

which formally reduces to 211, 212, 213, 157, 215

$$
V=k \Omega_{p}+\frac{D-p-1}{2} \sum_{i} \lambda_{i}^{\frac{1}{2}}
$$

where $\lambda_{i}$ run through the spectrum of the operator $L_{p}$.

The above general formula for static potential, Eq. (5.18), has been obtained making use of zeta-function regularization. We only remark that if $K_{p+1}\left(L_{p}\right)$ is not vanishing, the analytical continuation of $\zeta\left(s \mid L_{p}\right)$ contains a simple poles at $s=-1 / 2$. As a consequence, a contribution depending from the arbitrary scale parameter $\ell$ appears. We know that such a term is always absent for even $p$.

\subsubsection{The static potential on toroidal spaces}

As an application, we shall consider the Casimir energy of a toroidal $p$-brane evaluated in a spacetime with topology $\mathbb{R}^{D-p} \times T^{p}$. In this case, it is well known that the heat kernel expansion terminates to the Seeley-DeWitt coefficient $K_{p}$ and so there is no $\ell$ ambiguity in the static potential. Moreover, the $\zeta\left(s \mid L_{p}\right)$ reduces to the Epstein $Z$-function (see Eq. (3.2)). Making use of Eq. (5.18) and the functional relation for the Epstein $Z$-function (see Eq. (D.6) in Appendix D) we may rewrite the regularized potential as

$$
V_{\text {reg }}=\Omega_{p}\left[k-\frac{D-p-1}{2} \frac{\Gamma\left(\frac{p+1}{2}\right)}{\left(4 \pi^{3}\right)^{\frac{p+1}{2}}} Z_{\mathcal{R}_{p}}\left(\frac{p+1}{p} ; 0,-\vec{g}\right)\right],
$$

where $\Omega_{p}=(2 \pi)^{p} \operatorname{det} \mathcal{R}_{p}^{1 / 2}=\prod_{i=1}^{p} l_{i}$ is the volume of $T^{p}$. 
For an equilateral torus $r_{i}=r(i=1, \ldots, p)$, we find

$$
\begin{aligned}
V_{\text {reg }} & =k\left[(2 \pi r)^{p}-\frac{\alpha(\vec{g})}{r}\right] \\
\alpha(\vec{g}) & =\frac{D-p-1}{4 \pi k} \frac{\Gamma\left(\frac{p+1}{2}\right)}{\pi^{\frac{p+1}{2}}} Z_{I_{p}}\left(\frac{p+1}{p} ; 0,-\vec{g}\right),
\end{aligned}
$$

where $I_{p}$ is the identity matrix. For untwisted fields $\vec{X}^{\perp}(\vec{g}=0)$ and for any $p \in \mathbb{N}$ one has $Z_{I_{p}}\left(\frac{p+1}{p} ; 0,0\right)>0$ (see for example Refs. [219, 220, 126]). This means that for the untwisted toroidal $p$-brane $\alpha(0)>0$ (the Casimir forces are attractive) and the $p$-brane tends to collapse [157, 189, 215]. The behavior of an untwisted toroidal $p$-brane potential is similar to that of spherical $p$-branes [212, 213, 157]. On the other hand, for a twisted toroidal $p$-brane there are field sectors for which $\alpha(\vec{g})<0$. For example, if we choose $\vec{g} \equiv\left(\frac{1}{2}, \ldots, \frac{1}{2}\right)$, then for any $p \in \mathbb{N}$ $Z_{I_{p}}\left(\frac{p+1}{p} ; 0,-\frac{1}{2}\right)<0$ [219, 220, 126]. In this case the potential has a minimum at finite distance

$$
r_{0}=\left[\alpha\left(\frac{1}{2}, \ldots, \frac{1}{2}\right)\right]^{\frac{1}{p+1}}
$$

and its behaviour is similar to the potential of the open $p$-brane [211, 212, 213, 157].

Similar considerations hold for manifolds of the form $\mathcal{M}^{\sqrt{ }}=\mathcal{T}^{\mathcal{K}} \times \mathcal{M}^{\mathcal{Q}}$, with $p=K+Q$. It should be noted however that in this case, the $\ell$ ambiguity term may be present. With regard to this fact we say only few things. Since the underlying theory is not renormalizable, it is not possible to determine such parameter in the usual way. Furthermore, we are only considering the semiclassical (one-loop) approximation. Following Ref. [95], the dimensional parameter $\ell$ may phenomenologically summarize the unknown physics related to the approximations made. In this context, it should be determined experimentally. 


\section{$6 \quad$ Asymptotic properties of $p$-brane quantum state density}

In order to study the statistical properties of extended objects (we shall be mainly interested in (super)strings), it is necessary to have informations on the asymptotics of the density of states. In the case of field theory (point-like objects), these informations can be obtained from the heat-kernel expansion, which we have illustrated in some detail in Sec. 2.2.1, by making use of the Karamata tauberian theorem. One has to consider the leading term (Weyl term) and the result is the well known polynomial growing of the state density when the energy is going to infinity. For an extended object, the situation is more complicated and we need some preliminary mathematical results, which, in turn, are relevant in number theory. First, we shall discuss the analogue of heat-kernel expansion. Then, the Meinardus theorem will give us the asymptotics of the level degeneracy, which directly leads to the asymptotics of level state density for a generic extended object.

\subsection{Asymptotic properties of generating functions}

We have seen that the semiclassical quantization of a $p$-brane in $\left(S^{1}\right)^{p} \times \mathbb{R}^{D-p}$, is equivalent to deal with to the following "proper time Hamiltonian":

$$
L=\vec{p}^{2}+M^{2}
$$

where the mass operator $M^{2}$ is linearly related to the total number operator

$$
N=\sum_{i=1}^{d} \sum_{\vec{n} \in \mathbb{Z}^{p} /\{0\}} \omega_{\vec{n}} N_{\vec{n}}^{i} .
$$

Here $d=D-p-1$ and the frequencies are given by

$$
\omega_{\vec{n}}^{2}=\sum_{i-1}^{p}\left(\frac{2 \pi n_{i}}{l_{i}}\right)^{2},
$$

with the compactification lenghts $l_{i}=2 \pi r_{i}, i=1, \ldots, p$. The number operators $N_{\vec{n}}$ with $\vec{n}=$ $\left(n_{1}, \ldots, n_{p}\right) \in \mathbb{Z}^{p}$ and the commutation relations for the oscillators can be found for example in Refs. [221, 222, 188, 223, 224, 189]. Due to the linear Regge trajectory relation, one may deal with the number operator $N$. Furthermore, the flat particle Laplace operator $\vec{p}^{2}$ commutes with the mass operator. As a consequence, one may consider the trace of the heat number operator $\exp (-t N)$, the trace being computed over the entire Fock space, namely

$$
\mathcal{Z}(t)=\operatorname{Tr} e^{-t N}=\prod_{j}\left[1-e^{-t \omega_{j}}\right]^{-d},
$$

where $t>0$. For $p=1$ (string case) the function $\mathcal{Z}(z)$ of the complex variable $z=t+i x$ is known as the generating function of the partition function, which is well studied in the mathematical literature [225]. The properties of this generating function have been used to evaluate the asymptotic state density behaviour for $p=1$ [226, 4, 227, 228].

In the following, for the sake of completeness, we shall present some mathematical results we shall use in investigating the heat-kernel expansion of the operator $N$ [229]. We shall be interested in the asymptotics of the partition functions which admit an infinite product as associated generating function. We shall present a general theorem due to Meinardus [230, 231] following Ref. [232] (see also Ref. [233]) and in particular we shall discuss the so called vector-like partition functions, which are relevant in the determination of the asymptotic state density of quantum $p$-branes. 
Let us introduce the generating function

$$
f(z)=\prod_{n=1}^{\infty}\left[1-e^{-z n}\right]^{-a_{n}}=1+\sum_{n=1}^{\infty} v(n) e^{-z n},
$$

where $\operatorname{Re} z>0$ and $a_{n}$ are non-negative real numbers. Let us consider the associated Dirichlet series

$$
D(s)=\sum_{n=1}^{\infty} a_{n} n^{-s}, \quad s=\sigma+i t,
$$

which converges for $0<\sigma<p$. We assume that $D(s)$ can be analytically continued in the region $\sigma \geq-C_{0}\left(0<C_{0}<1\right)$ and here $D(s)$ is analytic except for a pole of order one at $s=p$ with residue $A$. Besides we assume that $D(s)=O\left(|t|^{C_{1}}\right)$ uniformly in $\sigma \geq-C_{0}$ as $|t| \rightarrow \infty$, where $C_{1}$ is a fixed positive real number. The following lemma [230, 231] is useful with regard to the asymptotic properties of $f(z)$ at $z=0$ :

Lemma 1 If $f(z)$ and $D(s)$ satisfy the above assumptions and $z=y+2 \pi i x$ then

$$
f(z)=\exp \left\{A \Gamma(p) \zeta_{R}(1+p) z^{-p}-D(0) \ln z+D^{\prime}(0)+O\left(y^{C_{0}}\right)\right\}
$$

uniformly in $x$ as $y \rightarrow 0$, provided $|\arg z| \leq \pi / 4$ and $|x| \leq 1 / 2$. Moreover there exists a positive number $\varepsilon$ such that

$$
f(z)=O\left(\exp \left\{A \Gamma(p) \zeta_{R}(1+p) y^{-p}-C y^{-\varepsilon}\right\}\right),
$$

uniformly in $x$ with $y^{\alpha} \leq|x| \leq 1 / 2$ as $y \rightarrow 0, C$ being a fixed real number and $\alpha=1+p / 2-p \nu / 4$, $0<\nu<2 / 3$.

Here is a sketch of the proof. The Mellin-Barnes representation of the function $\ln f(z)$ gives

$$
\ln f(z)=\frac{1}{2 \pi i} \int_{1+p-i \infty}^{1+p+i \infty} z^{-s} \zeta_{R}(s+1) \Gamma(s) D(s) d s .
$$

The integrand in the above equation has a first order pole at $s=p$ and a second order pole at $s=0$. Therefore shifting the vertical contour from $\operatorname{Re} z=1+p$ to $\operatorname{Re} z=-C_{0}$ (due to the conditions of the Lemma the shift of the line of integration is permissible) and making use of the theorem of residues one obtains

$$
\begin{aligned}
& \ln f(z)=A \Gamma(p) \zeta_{R}(1+p) z^{-p}-D(0) \ln z+D^{\prime}(0) \\
&+\frac{1}{2 \pi i} \int_{-C_{0}-i \infty}^{-C_{0}+i \infty} z^{-s} \zeta_{R}(s+1) \Gamma(s) D(s) d s .
\end{aligned}
$$

The first part of the Lemma follows from Eq. (6.4), since the absolute value of the integral in the above equation can be estimated to behave as $O\left(y^{C_{0}}\right)$. In a similar way one can prove the second part of the Lemma but we do not dwell on this derivation.

Now we are ready to state the main result, which permits to know the complete asymptotics of $v(n)$.

Theorem 4 (Meinardus) For $n \rightarrow \infty$ one has

$$
\begin{aligned}
& v(n)=C_{p} n^{\frac{2 D(0)-p-2}{2(1+p)}} \exp \left\{\frac{1+p}{p}\left[A \Gamma(1+p) \zeta_{R}(1+p)\right]^{\frac{1}{1+p}} n^{\frac{p}{1+p}}\right\}\left(1+O\left(n^{-k_{1}}\right)\right), \\
& C_{p}=\left[A \Gamma(1+p) \zeta_{R}(1+p)\right]^{\frac{1-2 D(0)}{2(1+p)}} \frac{e^{D^{\prime}(0)}}{[2 \pi(1+p)]^{\frac{1}{2}}}, \\
& k_{1}=\frac{p}{1+p} \min \left(\frac{C_{0}}{p}-\frac{\nu}{4}, \frac{1}{2}-\nu\right) .
\end{aligned}
$$


The proof of this theorem relies on the application of the saddle point method. Cauchy integral theorem gives

$$
v(n)=\frac{1}{2 \pi i} \int_{z_{0}}^{z_{0}+2 \pi i} f(z) e^{n z} d z=\int_{-1 / 2}^{1 / 2} f(y+2 \pi i x) e^{n(y+2 \pi i x)} d x .
$$

Since the maximum absolute value of the integral occurs for $x=0$, the Lemma implies that the integrand is well approximated by

$$
U=\exp \left[A \Gamma(p) \zeta_{R}(1+p) y^{-p}+n y\right] .
$$

Within the saddle point method one has to minimize this expression, i.e. $d U / d y=0$ and therefore

$$
y=n^{-\frac{1}{1+p}}\left[p A \Gamma(1+p) \zeta_{R}(1+p)\right]^{\frac{1}{1+p}} .
$$

The result of Meinardus theorem follows by carefully making the estimation of the integral (6.8) and making use of Eq. (6.9) (for an extensive account of the proof we refer the reader to Ref. [232]).

Coming back to our problem, we note that the quantity $\operatorname{Tr} e^{-t N}$ is a special kind of vector-like generating function, we are going to introduce. Let

$$
F(z)=\prod_{\vec{n} \in Z^{p} /\{0\}}\left[1-e^{-z \omega_{\vec{n}}}\right]^{-d},
$$

be a generating function, $\operatorname{Re} z>0, d>0$ and $\omega_{\vec{n}}$ given by Eq. (6.1). The theorem of Meinardus can be generalized to deal with a such vector valued function. In the formulation of this theorem the Dirichlet series $D(s)$ has been used (see the above discussion). In the case of the generating function (6.10), the role of $D(s)$ is played by the $p$-dimensional Epstein $Z$-function. More precisely, for $F(z)$ one obtains Eq. (6.2), but with $D(s)$ replaced by $d Z_{\mathcal{R}_{p}^{-1}}(s / p ; 0,0)$ and the residue $A$ replaced by $\pi^{p / 2} \operatorname{det} \mathcal{R}_{p}^{1 / 2} / \Gamma(1+p / 2)$, where $\mathcal{R}_{p}$ is the $p \times p$ diagonal matrix diag $\mathcal{R}=$ $\left(r_{1}^{2}, \ldots, r_{p}^{2}\right)$ (see Appendix $\mathrm{D}$ for definitions and properties).

Recalling that $Z_{\mathcal{R}}(0 ; 0,0)=-1$, we get the following asymptotic expansion for the function $\mathcal{Z}(t)$ (for small $t$ )

$$
\begin{aligned}
& \mathcal{Z}(t) \sim t^{d} \exp \left(\frac{d}{p} Z_{\mathcal{R}_{p}^{-1}}^{\prime}(0 ; 0,0)\right) \exp \left(B_{p} t^{-p}\right), \\
& B_{p}=\frac{2^{\frac{p}{2}} \pi^{p} d}{p} \Gamma\left(\frac{p}{2}\right) \zeta_{R}(p) \prod_{i=1}^{p} l_{i} .
\end{aligned}
$$

\subsection{Asymptotic density of $p$-brane quantum states}

In the following, the asymptotic behaviour of the degeneracy of the state density level related to a generic $p$-brane will be discussed. We have already mentioned that the knowledge of such asymptotic behaviour is important in the investigation of thermodynamical properties of extended objects. For ordinary matter fields, the leading term in the heat-kernel expansion, the Weyl term, determines the leading term of the density of states for large values of the energy. Here we would like to present the analogue of it for extended objects. For the sake of completeness we start with an elementary derivation of such asymptotic behaviour for small $t$.

In terms of $\mathcal{Z}$, the total number $q(n)$ of $p$-brane states may be described by

$$
\mathcal{Z}(t)=\sum_{n=0}^{\infty} q(n) e^{-z t} \text {. }
$$


Making use of the "thermodynamical methods" of Ref. [234], $\mathcal{Z}(t)$ may be regarded as a "partition function" and $t$ as the inverse "temperature". Thus, the related "free energy" $F_{t}$, "entropy" $S_{t}$ and "internal energy" $N_{t}$ may be written respectively as

$$
F_{t}=-\frac{1}{t} \ln \mathcal{Z}(t), \quad S_{t}=t^{2} \frac{\partial}{\partial t} F_{t}, \quad N_{t}=-\frac{\partial}{\partial t} \ln \mathcal{Z}(t) .
$$

The limit $n \rightarrow \infty$ corresponds to $t \rightarrow 0$. Furthermore, in this limit the entropy may be identified with $\ln q(n)$, while the internal energy is related to $n$. Hence, one has from Eq. (6.11)

$$
F_{t} \simeq-B_{p} t^{-p-1}, \quad S_{t} \simeq(p+1) B_{p} t^{-p}, \quad N_{t}=p B_{p} t^{-p-1} .
$$

Eliminating the quantity $t$ between the two latter equations one gets

$$
S_{t} \simeq \frac{p+1}{p}\left(p B_{p}\right)^{\frac{1}{p+1}} N_{t}^{\frac{p}{p+1}}
$$

As a result

$$
\ln q(n) \simeq \frac{p+1}{p}\left(p B_{p}\right)^{\frac{1}{p+1}} n^{\frac{p}{p+1}} .
$$

A more complete evaluation based on the result of Meinardus gives

$$
q(n) \sim C_{p} n^{X} \exp \left\{n^{\frac{p}{p+1}}\left(1+\frac{1}{p}\right)\left(p B_{p}\right)^{\frac{1}{p+1}}\right\},
$$

with definitions

$$
C_{p}=e^{\frac{d}{2} \zeta^{\prime}(0 \mid L)}(2 \pi(1+p))^{-\frac{1}{2}}\left(p B_{p}\right)^{\frac{1-2 d K_{0}}{2(1+p)}}, \quad X=\frac{2 d K_{0}-p-2}{2(p+1)} .
$$

In Eq. (6.12) the complete form of the prefactor appears.

Some remarks are in order. First of all let us consider the (super)string case. Then $p=1$ and for the open bosonic string Eqs. (6.12) and (6.13) give

$$
q(n)=C_{1} n^{-\frac{D+1}{4}} \exp \left\{\pi \sqrt{\frac{2 n(D-2)}{3}}\right\}\left(1+O\left(n^{-k_{1}}\right)\right),
$$

where the constant $C_{1}$ is given by

$$
C_{1}=2^{-\frac{1}{2}}\left(\frac{D-2}{24}\right)^{\frac{D-1}{4}} .
$$

The formulae (6.14) and (6.15) coincide with previous results for strings (see for example 226, 4, 235, 227, 228]). The new feature of this considerations is that the constant $C_{1}$ has been calculated now making use of Meinardus results.

The closed bosonic string can be dealt with by taking the constraint $N=\tilde{N}$ into account. As a result the total degeneracy of the level $n$ is simply the square of $q(n)$. In a similar way one can treat the open superstring. Furthermore using the mass formula $M^{2}=n$ (for the sake of simplicity here and in the following we assume a tension parameter, with dimensions of $(\text { mass })^{p+1}$, equal to 1 ) we find for the number of string states of mass $M$ to $M+d M$

$$
\nu_{1}(M) d M \simeq 2 C_{1} M^{\frac{1-D}{2}} \exp \left(b_{1} M\right) d M, \quad b_{1}=\pi \sqrt{\frac{2(D-2)}{3}} .
$$

One can show that the constant $b_{1}$ is the inverse of the Hagedorn temperature. It is also clear that the Hagedorn temperature can be obtained in a similar way for the other (super)string cases. 
Now let us consider the (super) $p$-brane case, namely $p>1$. Using again a linear mass formula $M^{2}=n$, Eqs. (6.12) and (6.13) lead to an asymptotic density of states of the form

$$
\begin{aligned}
& \nu_{p}(M) d M \simeq 2 C_{p} M^{\frac{2 p+1-2 D}{1+p}} \exp \left(b_{p} M^{\frac{2 p}{1+p}}\right), \\
& b_{p}=\frac{1+p}{p}\left[d A \Gamma(1+p) \zeta_{R}(1+p)\right]^{\frac{1}{1+p}} .
\end{aligned}
$$

This result has a universal character for all (super) $p$-branes. It was presented two decades ago in Refs. [234, 162] within the extended models for hadronic matter and more recently in Ref. [236]), but without the complete knowledge of the prefactor. The complete derivation presented here is contained in Ref. [229].

\subsection{Asymptotic density of parabosonic string quantum states}

Here we shall use the Meinardus theorem to evaluate the asymptotics for the level state density of parabosonic string. First, let us briefly recall the parastatistic idea.

It is quite standard nowadays to describe the quantum field theory in terms of operators obeying canonical commutation relations. However, there exists the alternative logical possibility of para-quantum field theory [237, 238], where parafields satisfy tri-linear commutation relations. Later, the paraquantization proposal was investigated in Ref. [239]. We also would like to remind that, in a general study of particle statistics within the algebraic approach to quantum field theory, parastatistics is one of the possibilities found in Refs. 240, 241. Despite the efforts to apply parastatistics for the description of internal symmetries (for example in paraquark models [238]) or even in solid state physics for the description of quasiparticles, no experimental evidence in favour of the existence of parafields has been found so far. Nevertheless, parasymmetry can be of some interest from the mathematical point of view. For example it can be considered as formal extension of the supersymmetry algebra. Furthermore, parasymmetry may find some physical application in string theory, where parastrings [242] have been constructed. It has been shown there, that these parastrings possess some interesting properties, like the existence of critical dimensions different from the standard ones, i.e. $D=10$ and 26 .

In the following, we shall briefly review the paraquantization for parabose harmonic oscillators, which are relevant to the parabosonic string in the limit $\wp \rightarrow \infty$, where $\wp$ is the order of the paraquantization. The Hamiltonian and the zero point energy for the free parabose system has the form

$$
\hat{H}=\sum_{n} \frac{\omega_{n}}{2}\left(a_{n}^{\dagger} a_{n}+a_{n} a_{n}^{\dagger}\right)-E_{0}, \quad E_{0}=\frac{\wp}{2} \sum_{n} \omega_{n} .
$$

The operators $a_{n}$ and $a_{n}^{\dagger}$ obey the following tri-commutation relations 237, 238]

$$
\left[a_{n},\left\{a_{m}^{\dagger}, a_{l}\right\}\right]=2 \delta_{n m} a_{l}, \quad\left[a_{n}\left\{a_{m}, a_{l}\right\}\right]=0 .
$$

The vacuum will be chosen to satisfy the relations

$$
a_{n}\left|0>=0, \quad\left\{a_{n}^{\dagger}, a_{m}\right\}\right| 0>=\wp \delta_{n m} \mid 0>,
$$

so that $\hat{H} \mid 0>=0$. The paracreation operators $a_{n}^{\dagger}$ do not commute and therefore the Fock space is quite complicated [238]. For $D$-dimensional harmonic oscillators $a_{n}^{i}$ of parabosonic string with frequencies $\omega_{n}^{i}=n$ we have the Hamiltonian

$$
\hat{H}=\sum_{i=1}^{D} \sum_{n=1}^{\infty} \frac{n}{2}\left\{a_{n}^{\dagger i}, a_{n}^{i}\right\}-E_{0} .
$$


A closed form for the partition function $\hat{Z}(t)=\operatorname{Tr} e^{-t \hat{H}}$, the trace being computed over the entire Fock space, in the limit $\wp \rightarrow \infty$ reads 243

$$
\hat{Z}(t)=\left\{\prod_{n=1}^{\infty} \frac{1}{\left(1-e^{-t n}\right)}\right\}^{D}\left\{\prod_{n, m=1}^{\infty} \frac{1}{1-e^{-t(n+m)}}\right\}^{\frac{D^{2}}{2}}\left\{\prod_{n=1}^{\infty}\left(1-e^{-2 n t}\right)\right\}^{\frac{D}{2}} .
$$

Our aim is to evaluate, asymptotically, the degeneracy or state level density corresponding to a parabosonic string, in the limit of infinite paraquantization order parameter. As a preliminary result, we need the asymptotic expansion of the partition function for $t \rightarrow 0$. To this aim, it may be convenient to work with the quantity

$$
F(t)=\ln \hat{Z}(t)=-D F_{1}(t)+\frac{D}{2} F_{1}(2 t)-\frac{D^{2}}{2} F_{2}(t),
$$

where we have introduced definitions

$$
F_{1}(t)=\sum_{n=1}^{\infty} \ln \left(1-e^{-t n}\right), \quad F_{2}(t)=\sum_{n, m=1}^{\infty} \ln \left(1-e^{-t(n+m)}\right) .
$$

With regards to the contributions $F_{1}(t)$, one may use a result, known in the theory of elliptic modular function as Hardy-Ramanujan formula [4], that is

$$
F_{1}(t)=-\frac{\pi^{2}}{6 t}-\frac{1}{2} \ln \frac{t}{2 \pi}+\frac{t}{24}+F_{1} \frac{4 \pi^{2}}{t} .
$$

Let us now consider the quantity $F_{2}(t)$. A Mellin representation gives

$$
\ln \left(1-e^{-t a}\right)=-\frac{1}{2 \pi i} \int_{\operatorname{Re} z=c>2} \Gamma(z) \zeta_{R}(1+z)(a t)^{-z} d z .
$$

As a result,

$$
F_{2}(t)=-\frac{1}{2 \pi i} \int_{\operatorname{Re} z=c>2} \Gamma(z) \zeta_{R}(1+z) \zeta_{2}(z) t^{-z} d z,
$$

where $\zeta_{2}(z) \equiv \sum_{n, m=1}^{\infty}(n+m)^{-z}$. Now it is easy to show that (see for example Ref. [244]) $\zeta_{2}(z)=\zeta_{R}(z-1)-\zeta_{R}(z)$ and so we have $F_{2}(t)=G_{2}(t)-F_{1}(t)$, where we have set

$$
G_{2}(t)=-\frac{1}{2 \pi i} \int_{\operatorname{Re} z=c>2} \Gamma(z) \zeta(z+1) \zeta(z-1) t^{-z} d z=\sum_{n=1}^{\infty} \ln \left(1-e^{-t n}\right)^{n},
$$

and the related generating function reads

$$
g_{2}(t)=\prod_{n=1}^{\infty}\left(1-e^{-t n}\right)^{n}
$$

For the estimation of the small $t$ behaviour, let us apply the results of Sec. 6.1 to the generating function $g_{2}(t)$. We have $D(s)=-\zeta_{R}(s-1), \alpha=2$, and $A=-1$. According to Meinardus lemma we arrive at the asymptotic expansions

$$
\begin{aligned}
& G_{2}(t) \simeq-\zeta_{R}(3) t^{-2}-\frac{1}{12} \ln t-\zeta_{R}^{\prime}(-1)+O(t), \\
& F(t) \simeq \frac{D^{2}}{2} \zeta_{R}(3) t^{-2}+\ln \left(\pi^{\frac{D}{4}}(2 \pi)^{\frac{D(D-2)}{4}} t^{\frac{6 D-5 D^{2}}{24}}\right)+\frac{D^{2}}{2} \zeta_{R}^{\prime}(-1)+O\left(t^{-1}\right), \\
& \hat{Z}(t) \simeq A t^{B} \exp \left(C t^{-2}\right),
\end{aligned}
$$


where in the latter equation

$$
A=\pi^{\frac{D}{4}}(2 \pi)^{\frac{D(D-2)}{4}} e^{\frac{D^{2}}{2} \zeta_{R}^{\prime}(-1)}, \quad B=\frac{6 D-5 D^{2}}{24}, \quad C=\frac{D^{2}}{2} \zeta_{R}(3) .
$$

Note that in ordinary string theory, the asymptotic behaviour of $Z_{1}(t)$ is of the kind $\exp \left(c t^{-1}\right)$.

Now, the degeneracy or density of levels can be easily calculated starting from the above asymptotic behaviour. In fact the density $\hat{v}(n)$ of levels for parabosonic strings (for a general discussion on parastrings see Ref. 242]) may be defined by

$$
\hat{Z}(z)=\operatorname{Tr} e^{-z \hat{H}}=1+\sum_{n=1}^{\infty} \hat{v}(n) e^{-z n} .
$$

The Cauchy theorem gives

$$
\hat{v}_{n}=\frac{1}{2 \pi i} \oint e^{z n} \hat{Z}(z) d z,
$$

where the contour integral is a small circle about the origin. For $n$ very large, the leading contribution comes from the asymptotic behaviour for $z \rightarrow 0$ of $\hat{Z}(z)$. Thus, making use of the Eq. (6.18) we may write

$$
\hat{v}_{n} \simeq \frac{A}{2 \pi i} \oint z^{B} e^{z n+C z^{-2}} d z .
$$

A standard saddle point evaluation (or Meinardus main theorem) gives as $n \rightarrow \infty$

$$
\hat{v}_{n} \simeq \hat{C}_{1} n^{-\frac{B+2}{3}} \exp \left(\hat{b}_{1} n^{\frac{2}{3}}\right),
$$

with $B$ as in Eq. 6.19), $\hat{b}_{1}=\frac{3}{2}\left(D^{2} \zeta(3)\right)^{\frac{1}{3}}$ and

$$
\hat{C}_{1}=\frac{1}{\sqrt{6}} 2^{\frac{D(D-2)}{4}} \pi^{\frac{(D+1)(D-2)}{4}} e^{\frac{D^{2}}{2} \zeta_{R}^{\prime}(-1)}\left[D^{2} \zeta_{R}(3)\right]^{\frac{6(D-2)-5 D^{2}}{72}} .
$$

Eqs. (6.18), (6.19), (6.20) and (6.21) have been obtained in [245]. The factor $\hat{b}_{1}$ is in agreement with result in Ref. 243], where however the prefactor $\hat{C}_{1}$ was missing. Here, with the help of Meinardus techniques, we have been able to compute it.

The asymptotic behaviour given by Eq. (6.20) should be compared with the one of the ordinary bosonic string and $p$-brane which we have discussed in previous subsections. As a consequence, one may conclude that the parabosonic string, in the limit of infinite paraquantization parameter, behaves as an ordinary membrane $(p=2)$. We will see that there is some indication that canonical partition function for $p$-branes does not exist. Thus, with regard to Hagedorn temperature, the situation for parabosonic strings may be similar to membranes. Hence, the concept of Hagedorn temperature is likely to be meaningless for parastrings.

\subsection{Extented objects and black holes}

Recently, spacetimes with black $q$-brane solutions, namely singular spacetimes for which the region of singularity assumes the shape of a $q$-brane, have been constructed [246]. Such solutions have attracted much attention in view of the fact that they can represent vacuum solutions of the 10-dimensional superstring for which $q=10-D$ is the dimensionality of an embedded flat space. The problem of finding black $q$-brane solutions of the 10-dimensional superstring theory can be reduced to the problem of finding black hole solutions to the Einstein equations in $D$-dimensions [246, 247]. These solutions have been used to study the statistical mechanics of black holes using the microcanonical ensemble prescription, this prescription being the unique 
reasonable framework for analyzing the problem [248, 249, 250]. In Refs. [249, 250] the approximate semiclassical formula for the neutral black hole degeneracy $\rho(M)$ of states at mass level $M$ has been obtained, which reads

$$
\rho(M) \sim B(M) \exp \left(S_{E}(M)\right) .
$$

Here $S_{E}$ is the Euclidean action (the so called Bekenstein-Hawking entropy)

$$
S_{E}(M)=\sqrt{\pi}\left[\frac{2^{2 D-3} \Gamma\left(\frac{D-1}{2}\right) G}{(D-2)^{D-2}}\right]^{\frac{1}{D-3}} M^{\frac{D-2}{D-3}},
$$

$G$ the generalized Newton constant and the prefactor $B(M)$ represents general quantum field theoretical corrections to the state density. To begin with, firstly, let us consider the quantity $S_{E}(M)$. For $D=4$, it reduces to the well known result [251, 252]

$$
S_{E}(M)=4 \pi G M^{2} \text {. }
$$

The exponential factor of the statistical mechanical density of states (degeneracies) for black holes given in Eqs. (6.23) reveals great similarities with the corresponding exponential factor of the density $\nu_{p}(M)$ of states of quantum $p$-branes derived above. For this reason it has been proposed that black holes might be considered as quantum extended objects like $p$-branes [249, 250]. In fact, the comparison of Eqs. (6.16) and (6.22) yields

$$
p=\frac{D-2}{D-4} .
$$

The only integer solutions of Eq. (6.24) are $p=1(D=\infty)$, the string case corresponds to an infinite dimensional black hole, $p=2(D=6), p=3(D=5)$ and the limit $p \rightarrow \infty$ corresponding to the 4-dimensional black hole. This last result has been pointed out in Ref. [236].

It should be noted that for any fixed $p$, the density of states of a $p$-brane grows slower than the one for 4-dimensional black holes, but faster than the one for strings. So the probability of a $p$-brane being in an interval of mass $(M, M+d M)$ increases with the mass and the total probability diverges. In accordance with the argument of Ref. [248] this divergence indicates a breakdown of the canonical ensemble.

For super $p$-branes the asymptotic behaviour of the state density looks just in the same way. We have seen in the previous subsection that the variation of the Wess-Zumino-Witten term of the globally supersymmetric $p$-brane action is a total derivative if and only if the $\Gamma$-matrix identity holds for arbitrary spinors. Then there exist only several admissible pairs $(p, D), 1 \leq$ $p \leq 5$, associated with the four composition-division algebras for each spinor type [185, 186, 171]. Furthermore, the inclusion of winding modes can be done. The value of this function at $s=0$ does not depend on winding numbers $g_{i}$, but its derivative in general depends on them. Therefore the total degeneracy of the level $n$ and the density of states change in the presence of winding modes.

Finally a warning about the possible identification between $p$-branes and black holes. The first trivial observation stems from the fact that the asymptotic behaviour of two functions does not lead to the conclusion that they are similar. The second one is more subtle. If one naively assume that black holes share, asymptotically, some properties related to quantum extended objects, it is a fact that the nature of these extended object may not be determined by their asymptotic behaviour. In fact, in the previous subsection, we have seen that a parabosonic string, asymptotically, behaves as a membrane. Thus the two original physical systems are quite different from the physical view point, even though they asymptotically have the same behaviour. Finally, there is a third serious fact against the identification of black holes and $p$-branes. As we have shown, for the $p$-branes, the prefactor $B_{p}(M)$ is computable and is finite. For the black 
holes, however, as first pointed out in Ref. [253], the prefactor $B(M)$ seems untractable and turns out to be divergent and this divergence may be regarded as the first quantum correction to the Bekenstein-Hawking entropy (see, for example, 254, 255, 256, 257, 258].)

These divergences appear also in the so called "entanglement or geometric entropy" 259, 260, 261, 262] and are peculiar of space-times with horizons and, in these cases, a possible physical origin of them can be traced back to the equivalence principle [263, 264, 265]. 


\section{Finite temperature quantum properties on ultrastatic space- time with hyperbolic spatial section}

In this section we are going to study some properties of free and self interacting scalar fields on ultrastatic manifolds $\mathcal{M}^{\mathcal{D}}$ with hyperbolic spatial section. In particular we shall give general expressions for free energy and thermodynamic potential and, for the more important 4dimensional case $\mathcal{M}^{\triangle}$, we shall also give low and high temperature expansion of those quantities and we shall discuss in some detail finite temperature effective potential and Bose-Einstein condensation. For the sake of completeness we shall also give free energy and thermodynamic potential for the cases in which the constant curvature spatial section is $T^{N}$ and $S^{N}$.

\subsection{The free energy and thermodynamic potential}

We recall that the complete high temperature expansion for the thermodynamic potential of a relativistic ideal Bose gas was derived and discussed in Refs. [266, 80, 267]. The extension of the formula to a Fermi gas was given first in Ref. [78]. The expansions mentioned above have been done with either trivial (that is $\mathbb{R}^{N}$ ) and toroidal topology. Generalizations of the high temperature expansion to particular curved spaces have been given in Refs. [59, 268, 269]. In Refs. [59, 268] also spin $1 / 2$ fields have been discussed. Only recently space-times with hyperbolic spatial sections have been considered for this purpose [79, 270, 271, 272].

Here we present the evaluation of the thermodynamic potential for massive scalar fields in thermal equilibrium at finite temperature $T=1 / \beta$ on an ultrastatic space-time with constant curvature spatial section of the kind $T^{N}, S^{N}$ and $H^{N} / \Gamma$. Of course, for arbitrary $N, \Gamma$ is assumed to contain only hyperbolic elements. Only for $N=3$ and $N=2$ elliptic elements shall be taken into account.

At very high temperatures, we expect the dominant term of the thermodynamic energy to be insensitive to curvature, because particles at high energy have wave length much smaller than the curvature radius. We have no physical ground for similar conclusions with respect to topological effects, if any. It must also be admitted that there is no clear definition of what a "topological effect" should be, because it is difficult to disentangle curvature from topology. Here we leave it understood that by non trivial topology we really mean the non triviality of the group $\Gamma$, i.e. $\Gamma \neq e$.

Free energy and thermodynamic potential have been defined in Sec. 2.6, where general integral representations valid on any curved ultrastatic manifold have been also given. Here we will specialize Eqs. 2.59 2.61) and 2.65 2.67) to torii, spheres and compact hyperbolic manifolds with elliptic and hyperbolic elements. Moreover, on these particular manifolds we shall be able to give another representation of finite temperature quantities in terms of Mc Donald functions, which is very useful in order to get the low temperature expansion.

The free energy can be derived from the thermodynamic potential in the limit $\mu \rightarrow 0$ and for this reason we directly attach the computation of thermodynamic potential, deriving the free energy as a particular case.

The thermodynamic potential on $\mathbb{R} \times T^{N}$. Here we use the notations of Sec. 3.2. There is no possibility to confuse the volume $\Omega_{N}$ with the thermodynamic potential $\Omega_{\beta}$. Using Eq. (3.1) in Eq. (2.66), after some calculations we obtain

$$
\begin{aligned}
\Omega_{\beta}^{T}\left(\beta, \mu \mid \mathrm{E}_{N}\right)=-\frac{2 \sqrt{2} \Omega_{N}}{\sqrt{\pi}} & \sum_{n=1}^{\infty} \sum_{\vec{k} \neq 0} \frac{\cosh n \beta \mu}{\sqrt{n \beta}} \\
& \times\left(\vec{k} \cdot \mathcal{R}_{N}^{-1} \vec{k}+\alpha^{2}\right)^{1 / 4} K_{1 / 2}\left(n \beta\left[\vec{k} \cdot \mathcal{R}_{N}^{-1} \vec{k}+\alpha^{2}\right]^{1 / 2}\right),
\end{aligned}
$$


where $\vec{k} \in \mathbb{Z}^{N}$ and for simplicity we have written the formula for untwisted fields. Replacing $\vec{k}$ with $\vec{k}+\vec{q}$ one obtains the formula for arbitrary twists. Of course, in the limit $\mu \rightarrow 0$ this gives the representation for the free energy. The latter equation is particularly useful if one is interested in the low temperature expansion. For high temperature expansion it is convenient to use another expression, which can be obtained by the Mellin-Barnes representation, Eq. (2.67).

The thermodynamic potential on $\mathbb{R} \times S^{N}$. As for the vacuum energy it is sufficient to compute the thermodynamic potential for $S^{1}$ and $S^{2}$ and then apply the recurrence relations for the $\zeta$-function, Eq. (3.15). In fact we have

$$
\frac{\Omega_{\beta}^{S}\left(\beta, \mu \mid \mathrm{E}_{N+2}\right)}{\Omega_{N+2}}=-\frac{1}{2 \pi N \Omega_{N}}\left[\left(\alpha^{2}+\kappa \varrho_{N}^{2}\right) \Omega_{\beta}^{S}\left(\beta, \mu \mid \mathrm{E}_{N}\right)-\zeta^{\prime}\left(-1 \mid \mathrm{E}_{N}\right)\right] .
$$

For $S^{1}$ we have simply to take Eq. (7.1) for $N=1$. So we get

$$
\Omega_{\beta}^{S}\left(\beta, \mu \mid \mathrm{七}_{1}\right)=-\frac{2 \sqrt{2} \Omega_{1}}{\sqrt{\pi}} \sum_{n=1}^{\infty} \sum_{k=-\infty}^{\infty} \frac{\cosh n \mu \beta}{\sqrt{n \beta}}\left(k^{2}+\alpha^{2}\right)^{1 / 4} K_{1 / 2}\left(n \beta \sqrt{k^{2}+\alpha^{2}}\right),
$$

while for $S^{2}$ we use Eq. (3.17) in Eq. (2.66). In this way the thermodynamic potential reads

$$
\Omega_{\beta}^{S}\left(\beta, \mu \mid \mathrm{E}_{2}\right)=-\frac{\Omega_{2}}{i \sqrt{2 \pi}} \sum_{n=1}^{\infty} \frac{\cosh n \beta \mu}{(n \beta)^{3 / 2}} \int_{\Gamma} \frac{K_{3 / 2}\left(n \beta \sqrt{z^{2}+\alpha^{2}}\right)\left(z^{2}+\alpha^{2}\right)^{3 / 4}}{\cos ^{2} \pi z} d z .
$$

The thermodynamic potential on $\mathbb{R} \times H^{N} / \Gamma$. In Sec. 2.6 three different representations of the temperature dependent part of the thermodynamic potential have been given by means of Eqs. (2.65-2.67). As we shall see in the following, such representations are useful for low and high temperature expansion. In order to specialize those equations to an ultrastatic space-time with a compact spatial section $H^{N} / \Gamma$, we separate the contributions coming from different kinds of elements of the isometry group and first compute the contributions due to the identity in the two cases $N=3$ and $N=2$.

Using Eqs. (2.66), (C.5) and (C.9) we obtain

$$
\begin{aligned}
& \Omega_{\beta}^{I}\left(\beta, \mu \mid \mathrm{七}_{3}\right)=-\frac{\alpha^{4} \Omega\left(\mathcal{F}_{3}\right)}{\pi^{2}} \sum_{n=1}^{\infty} \frac{\cosh n \beta \mu}{(n \beta \alpha)^{2}} K_{2}(n \beta \alpha), \\
& \Omega_{\beta}^{I}\left(\beta, \mu \mid \mathrm{E}_{2}\right)=-\frac{2 \Omega\left(\mathcal{F}_{2}\right)}{\sqrt{2 \pi}} \sum_{n=1}^{\infty} \frac{\cosh n \beta \mu}{(n \beta)^{3 / 2}} \int_{0}^{\infty} \frac{K_{3 / 2}\left(n \beta \sqrt{r^{2}+\alpha^{2}}\right)\left(r^{2}+\alpha^{2}\right)^{3 / 4}}{\cosh ^{2} \pi r} d r .
\end{aligned}
$$

As for the spherical case, from the recurrence relations for the $\zeta$-function, Eq. (3.52), we have the thermodynamic potential by means of equation

$$
\frac{\Omega_{\beta}^{I}\left(\beta, \mu \mid \mathrm{E}_{N+2}\right)}{\Omega\left(\mathcal{F}_{N+2}\right)}=-\frac{1}{2 \pi N \Omega\left(\mathcal{F}_{N}\right)}\left[\left(\alpha^{2}+\kappa \varrho_{N}^{2}\right) \Omega_{\beta}^{I}\left(\beta, \mu \mid \mathrm{E}_{N}\right)-\zeta_{I}^{\prime}\left(-1 \mid \mathrm{E}_{N}\right)\right] .
$$

The contribution due to hyperbolic elements can be computed in arbitrary dimensions using Eq. (3.54) or (3.50) in Eq. (2.65) or (2.66). So we obtain the two representations

$$
\begin{aligned}
& \Omega_{\beta}^{H}\left(\beta, \mu \mid \mathrm{E}_{N}\right)=\frac{1}{\pi} \sum_{n=1}^{\infty} \int_{-\infty}^{\infty} e^{i n \beta t} \ln Z\left(\varrho_{N}+\sqrt{[t+i \mu]^{2}+\alpha^{2}}\right) d t \\
& \Omega_{\beta}^{H}\left(\beta, \mu \mid \mathrm{E}_{N}\right)=-\frac{2 \alpha^{2}}{\pi} \sum_{n=1}^{\infty} \cosh n \beta \mu \sum_{\{\gamma\}} \sum_{k=1}^{\infty} \frac{\chi^{k}(\gamma) l_{\gamma}}{S_{N}\left(k ; l_{\gamma}\right)} \frac{K_{1}\left(\alpha \sqrt{(n \beta)^{2}+\left(k l_{\gamma}\right)^{2}}\right.}{\alpha \sqrt{(n \beta)^{2}+\left(k l_{\gamma}\right)^{2}}} .
\end{aligned}
$$


As we shall see in a moment, the latter equation is particularly useful in the low temperature expansion.

Finally, the contributions due to elliptic elements for $N=3$ and $N=2 \mathrm{read}$

$$
\begin{aligned}
& \Omega_{\beta}^{E}\left(\beta, \mu \mid \mathrm{E}_{3}\right)=-\frac{2 \alpha^{2} E}{\pi} \sum_{n=1}^{\infty} \frac{\cosh n \beta \mu}{n \beta \alpha} K_{1}(n \beta \alpha), \\
& \Omega_{\beta}^{E}\left(\beta, \mu \mid \mathrm{E}_{2}\right)=-\frac{1}{\pi} \sqrt{\frac{2}{\pi}} \sum_{n=1}^{\infty} \frac{\cosh n \beta \mu}{\sqrt{n \beta}} \int_{-\infty}^{\infty}\left(r^{2}+\alpha^{2}\right)^{1 / 4} K_{1 / 2}\left(n \beta \sqrt{r^{2}+\alpha^{2}}\right) E_{2}(r) d r .
\end{aligned}
$$

The free energy on $\mathbb{R} \times H^{N} / \Gamma$. Putting $\mu=0$ in Eqs. (7.2)-(7.5) we obtain the corresponding formulae for the free energy of a charged scalar field. In the case of a neutral scalar field, we have to multiply all results by a factor $1 / 2$. Here we simply write down the formulae of free energy for $N=3$ and $N=2$. They read

$$
\begin{aligned}
& F_{\beta}\left(\beta \mid \mathrm{E}_{3}\right)=-\nu \sum_{n=1}^{\infty}\{ \frac{\alpha^{4} \Omega\left(\mathcal{F}_{3}\right)}{\pi^{2}} \frac{K_{2}(n \beta \alpha)}{(n \beta \alpha)^{2}}+\frac{2 \alpha^{2} E}{\pi} \frac{K_{1}(n \beta \alpha)}{(n \beta \alpha)} \\
&\left.-\frac{2}{\pi} \int_{0}^{\infty} \cos n \beta t \ln Z\left(\varrho_{3}+\sqrt{t^{2}+\alpha^{2}}\right) d t\right\}, \\
& F_{\beta}\left(\beta \mid \mathrm{E}_{2}\right)=-\nu \sum_{n=1}^{\infty}\left\{\frac{\sqrt{2} \Omega\left(\mathcal{F}_{2}\right)}{\sqrt{\pi}} \int_{0}^{\infty} \frac{K_{3 / 2}\left(n \beta \sqrt{r^{2}+\alpha^{2}}\right)\left(r^{2}+\alpha^{2}\right)^{3 / 4}}{(n \beta)^{3 / 2} \cosh ^{2} \pi r} d r\right. \\
&+\frac{2^{3 / 2}}{\pi^{3 / 2}} \int_{0}^{\infty} \frac{K_{1 / 2}\left(n \beta \sqrt{r^{2}+\alpha^{2}}\right)\left(r^{2}+\alpha^{2}\right)^{1 / 4} E_{2}(r)}{\sqrt{n \beta}} d r \\
&\left.\quad-\frac{2}{\pi} \int_{0}^{\infty} \cos n \beta t \ln Z\left(\varrho_{2}+\sqrt{t^{2}+\alpha^{2}}\right) d t\right\} .
\end{aligned}
$$

In the rest of this section we focus our attention on a 4-dimensional space-time with a hyperbolic spatial part. Then we simplify the notation using $\mathcal{F}$ in place of $\mathcal{F}_{3}$ and we leave to drop the argument of the functions. For example, $\Omega_{\beta}$ has to be understood as $\Omega_{\beta}\left(\beta, \mu \mid \mathrm{E}_{3}\right)$. We also substitute $M$, the effective mass, in place of $\alpha$.

The low temperature expansion of thermodynamic potential on $\mathbb{R} \times H^{3} / \Gamma$. Recalling that for real values of $z$ and $\nu$ the asymptotics for Mc Donald functions reads

$$
K_{\nu}(z) \sim \sqrt{\frac{\pi}{2 z}} e^{-z} \sum_{k=0}^{\infty} \frac{\Gamma(\nu+k+1 / 2)}{\Gamma(k+1) \Gamma(\nu-k+1 / 2)}(2 z)^{-k}
$$

and taking Eqs. (7.2), (7.3) and (7.4) into account we get the low temperature expansion

$$
\begin{array}{r}
\Omega_{\beta} \sim-\sum_{n=1}^{\infty} \sum_{\{\gamma\}} \sum_{j=1}^{\infty} \frac{\chi^{j}(\gamma) l_{\gamma}}{S_{3}\left(j ; l_{\gamma}\right)} \frac{M^{2} e^{-n \beta\left[M \sqrt{1+\left(j l_{\gamma} / n \beta\right)^{2}}-|\mu|\right]}}{(2 \pi)^{1 / 2}\left(M \sqrt{n^{2} \beta^{2}+j^{2} l_{\gamma}^{2}}\right)^{3 / 2}} \\
\times \sum_{k=0}^{\infty} \frac{\Gamma(k+3 / 2)\left(2 M \sqrt{n^{2} \beta^{2}+j^{2} l_{\gamma}^{2}}\right)^{-k}}{\Gamma(k+1) \Gamma(-k+3 / 2)} \\
-\frac{M^{2} E}{(2 \pi)^{1 / 2}} \sum_{n=1}^{\infty} \frac{e^{-n \beta(M-|\mu|)}}{(n \beta M)^{3 / 2}} \sum_{k=0}^{\infty} \frac{\Gamma(k+3 / 2)(2 n \beta M)^{-k}}{\Gamma(k+1) \Gamma(-k+3 / 2)} \\
-\frac{M^{4} \Omega(\mathcal{F})}{(2 \pi)^{3 / 2}} \sum_{n=1}^{\infty} \frac{e^{-n \beta(M-|\mu|)}}{(n \beta M)^{5 / 2}} \sum_{k=0}^{\infty} \frac{\Gamma(k+5 / 2)(2 n \beta M)^{-k}}{\Gamma(k+1) \Gamma(-k+5 / 2)} .
\end{array}
$$


It has to be noted that the leading terms come from the topological part since they dominate the volume part by one power of $1 / \beta$. This is in accord with the intuitive expectation that at very low temperature the quantum field is probing the full manifold because the most occupied states are the low energy ones. The last term of the latter formula, which does not contain topological contributions, gives the correct non relativistic limit if we set $\mu_{N R}=|\mu|-M$ for the non-relativistic chemical potential.

The high temperature expansion of thermodynamic potential on $\mathbb{R} \times H^{3} / \Gamma$. In order to derive the high temperature expansion, it is convenient to use the Mellin-Barnes representation, Eq. (2.67) and integrate it on a closed path enclosing a suitable number of poles. To carry out the integration we recall that the $\zeta$-function in 3-dimensional compact manifold without boundary has simple poles at the points $s=3 / 2-k(k=0,1, \ldots)$ and simple zeros at the points $s=0,-1,-2, \ldots$ 229. On the other hand, $\zeta_{R}(s)$ has a simple pole at $s=1$ and simple zeros at all the negative even numbers. Then the integrand function

$$
\Gamma(s+2 n-1) \zeta_{R}(s) \zeta\left(\frac{s+2 n-1}{2} \mid \mathrm{E}_{3}\right) \beta^{-s},
$$

has simple poles at the points $s=1$ and $s=-2(n+k)(k=0,1,2, \ldots)$. Moreover, for $n=2$ we have another simple pole at $s=0$, for $n=1$ at $s=0$ and $s=2$ and finally for $n=0$ at $s=0,2,4$. In the latter case $s=0$ is a double pole. Hence integrating this function and recalling that in our case the residues of $\zeta\left(s \mid \mathrm{E}_{3}\right)$ are given by Eq. (C.13) we obtain the high temperature expansion

$$
\begin{aligned}
& \Omega_{\beta}=-\frac{\Omega(\mathcal{F}) \pi^{2}}{45} \beta^{-4}+\left[\frac{\Omega(\mathcal{F})}{12}\left(M^{2}-2 \mu^{2}\right)-\frac{\pi E}{3}\right] \beta^{-2} \\
&-\left\{\frac{\Omega(\mathcal{F})\left(M^{2}-\mu^{2}\right)^{3 / 2}}{6 \pi}-E\left(m^{2}-\mu^{2}\right)^{1 / 2}\right. \\
&\left.+\sum_{n=0}^{\infty} \frac{\mu^{2 n}}{\sqrt{\pi} n !} \sum_{\{\gamma\}} \sum_{j=1}^{\infty} \frac{\chi^{j}(\gamma) l_{\gamma}}{S_{3}\left(j ; l_{\gamma}\right.}\left[\frac{j l_{\gamma}}{2 M}\right]^{n-1 / 2} K_{n-1 / 2}\left(M j l_{\gamma}\right)\right\} \beta^{-1} \\
&-\left(\ln \frac{M \beta}{4 \pi}+\gamma-\frac{3}{4}\right)\left[\frac{M^{4} \Omega(\mathcal{F})}{16 \pi^{2}}-\frac{M^{2} E}{2 \pi}\right]-\mu^{2}\left[\frac{M^{2} \Omega(\mathcal{F})}{8 \pi^{2}}-\frac{E}{2 \pi}\right]+\mu^{4} \frac{\Omega(\mathcal{F})}{24 \pi^{2}} \\
&-\frac{M^{2} E}{4 \pi}-\frac{M^{2}}{\pi} \sum_{\{\gamma\}} \sum_{j=1}^{\infty} \frac{\chi^{j}(\gamma) l_{\gamma}}{S_{3}\left(j ; l_{\gamma}\right.} \frac{K_{1}\left(M j l_{\gamma}\right)}{M j l_{\gamma}}+O\left(\beta^{2}\right), \\
& O\left(\beta^{2}\right) \sim-\sum_{k=1}^{\infty} \beta^{2 k} \zeta_{R}^{\prime}(-2 k) \sum_{n=0}^{k} \frac{\mu^{2 n}}{(2 n) !(k-n) !} \times \\
& {\left[\frac{\Omega(\mathcal{F}) M^{4}}{16 \pi^{2}(k-n+2) !}-\frac{E M^{2}}{\pi(k-n+1) !}\right]\left(\frac{M}{2}\right)^{2(k-n)}, }
\end{aligned}
$$

which is in agreement with results of Ref. [80] in the case of a flat and topological trivial space.

From the high-temperature expansion we see that hyperbolic elements enter the thermodynamic potential and the free energy with two main contributions, one linear in $T=1 / \beta$ and one independent of $T$. In sharp contrast are placed elliptic elements, which enter the formulae in all terms of the expansion, but the leading one. Even with zero chemical potential, the elliptic number gives a negative contribution to the coefficient of $T^{2}=1 / \beta^{2}$. Thus the pattern of symmetry breaking in the finite temperature effective potential will be probably modified by the elliptic topology of the manifold. 
By looking at Eq. (7.10), we also note that $\Omega_{\beta}$ in the complex $\mu$-plane presents, as expected, two branch points at $|\mu|=M$ in agreement with flat-space results.

Another form of the high temperature expansion which looks quite similar to the one which one has on a flat space-time, can derived by means of Eq. 2.68) (see Ref. 273]).

The results here obtained can also be extended to fermions once a given spin structure has been chosen on the manifold, the different spin structures being parametrized by the first cohomology group $H^{1}\left(H^{3} / \Gamma, \mathbb{Z}_{2}\right)$. The expansion for fermions can be obtained using the relation $\Omega_{f}(\beta, \mu)=2 \Omega_{b}(2 \beta, \mu)-\Omega_{b}(\beta, \mu)$ [59], where $f / b$ stands for fermion/boson degrees of freedom, by referring to our previous results.

\subsection{The Bose-Einstein condensation on $\mathbb{R} \times H^{3}$}

As a first application of the formalism we have developed in this section we discuss BoseEinstein condensation for a non relativistic ideal gas. The physical phenomenon, which in the non relativistic case has a long story [274], is well described in many text books (see for example Ref. [275]) and a rigorous mathematical discussion of it was given by many authors [276, 277, 278]. The generalization to a relativistic ideal Bose gas is non trivial and only recently has been discussed in a series of papers [266, 30, 267, 269]. The effect of self-interaction has been taken into account, at least in the one-loop approximation with a proper relativistic treatment [279, 280] and the interesting interpretation of Bose-Einstein condensation as a symmetry breaking effect both in flat as well as in curved space-time has been analyzed [267, 279, 281]. Related and recent works on Bose-Einstein condensation in curved space-times have been presented in Refs. 282, 283, 284

It is well known that in the thermodynamic limit (infinite volume and fixed density) there is a phase transition of the first kind in correspondence of the critical temperature at which the condensation manifests itself. At that temperature, the first derivative of some continuous thermodynamic quantities has a jump. If the volume is kept finite there is no phase transition, nevertheless the phenomenon of condensation still occurs, but the critical temperature in this case is not well defined.

As an example here we focus our attention on $\mathbb{R} \times H^{3}$. We shall derive the thermodynamic potential for a free, charged scalar field of mass $m$. As we know, the thermodynamic potential has two branch points when the chemical potential $\mu$ reaches $\pm \omega_{0}, \omega_{0}^{2}=M^{2}=m^{2}+|\kappa|$ being the lower bound of the spectrum of the operator $\mathrm{E}_{3}=-\Delta_{3}+m^{2}$ and $\kappa$ the negative constant curvature of $H^{3}$. The values $\pm \omega_{0}$ will be reached by $\mu=\mu(T)$ of course for $T=0$, but also for some $T=T_{c}>0$. This is the critical temperature at which the Bose gas condensates.

The elementary properties of the Laplace-Beltrami operator on $H^{N}$, that is the spectrum and the density of states has been derived in Sec. 3.3.1. In particular, for the density of zero angular momentum radial functions we have found $\Phi_{3}(r)=r^{2} / 2 \pi^{2}$. The continuum spectrum of the Laplacian on $H^{3}$ has a lower bound at $\lambda=1$ (or $\lambda=|\kappa|$ in standard units) in contrast with which in general happens on the compact manifold $H^{3} / \Gamma$. We also notice that the wave operator propagates the field excitations on the light cone, hence the gap should not be interpreted as a physical mass.

In the following, we shall need low and high temperature expansions of thermodynamic potential. Then we shall use Eqs. (7.8) and (7.10) disregarding topological contributions. As usual, in order to avoid divergences we shall consider a large volume $\Omega$ in $H^{3}$ and the limit $\Omega \rightarrow \infty$ shall be understood when possible. 
The Bose-Einstein condensation. In order to discuss Bose-Einstein condensation we have to analyze the behaviour of the charge density

$$
\varrho=-\frac{\partial \Omega_{\beta}(\beta, \mu)}{\partial \mu}=-\frac{\beta z}{\Omega} \frac{\partial \Omega_{\beta}(\Omega, \beta, z)}{\partial z} \equiv f(z)-f(1 / z)
$$

in the infinite volume limit. Here $z=\exp \beta \mu$ is the activity and

$$
f(z)=\sum_{j} \frac{z}{\Omega\left(e^{\beta \omega_{j}}-z\right)} .
$$

The $\omega_{j}$ in the sum are meant to be the Dirichlet eigenvalues for any normal domain $\Omega \subset H^{3}$. That is, $\Omega$ is a smooth connected submanifold of $H^{3}$ with non empty piecewise $C^{\infty}$ boundary. By the infinite volume limit we shall mean that a nested sequence of normal domains $\Omega_{k}$ has been chosen together with Dirichlet boundary conditions and such that $\bigcup_{k} \Omega_{k} \equiv H^{3}$. The reason for this choice is the following theorem (see for example [114):

Theorem 5 (Mc Kean) if $\omega_{0}^{k}$ denotes the smallest Dirichlet eigenvalue for any sequence of normal domains $\Omega_{k}$ filling all of $H^{3}$ then $\omega_{0}^{k} \geq M$ and $\lim _{k \rightarrow \infty} \omega_{0}^{k}=M$.

Although the above inequality is also true for Neumann boundary conditions, the existence of the limit in not assured to the authors knowledge.

Now we can show the convergence of the finite volume activity $z_{k}$ to a limit point $\bar{z}$ as $k \rightarrow \infty$. To fix ideas, let us suppose $\varrho \geq 0$ : then $z_{k} \in\left(1, \exp \beta \omega_{0}^{k}\right)$. Since $\varrho(\Omega, \beta, z)$ is an increasing function of $z$ such that $\varrho(\Omega, \beta, 1)=0$ and $\varrho(\Omega, \beta, \infty)=\infty$, for each fixed $\Omega_{k}$ there is a unique $z_{k}(\bar{\varrho}, \beta) \in\left(1, \beta \exp \omega_{0}^{k}\right)$ such that $\bar{\varrho}=\varrho\left(\Omega_{k}, \beta, z_{k}\right)$. By compactness, the sequence $z_{k}$ must have at least one fixed point $\bar{z}$ and as $\omega_{0}^{k} \rightarrow M^{2}$ as $k$ goes to infinity, by Mc Kean theorem, $\bar{z} \in[1, \exp \beta M]$.

From this point on, the mathematical analysis of the infinite volume limit exactly parallels the one in flat space for non relativistic systems, as it is done for example in Refs. [277, 285, 278]. An accurate analysis for the relativistic ideal gas in Minkowski space can be found in Ref. [266]. The difference between $R \times H^{3}$ and $R^{4}$ is simply due to the fact that the mass $m$ is replaced by $M$. For this reason, here we skip all details of computation and refer the reader to the literature.

We recall that there is a critical temperature $T_{c}$, related to $\varrho$ by a complicated integral equation, over which there are no particles in the ground state. The solution of such an equation states that for $T>T_{c}$ one always has $|\mu|<M$ and a vanishing charge density $\varrho_{0}$ of the particles in the ground state, while for $T \leq T_{c}|\mu|$ remains equal to $M$ and the charge density of the particles in the ground state is non vanishing. That is, below $T_{c}$ one has Bose-Einstein condensation.

The critical temperature can be easily obtained in the two cases $\beta M \gg 1$ and $\beta M \ll 1$ (in the case of massive bosons these correspond to non relativistic and ultrarelativistic limits respectively). In fact one has

$$
\begin{array}{ll}
T_{c}=\frac{2 \pi}{M}\left(\frac{\varrho}{\zeta_{R}(3 / 2)}\right)^{2 / 3}, \quad \beta M \gg 1, \\
T_{c}=\left(\frac{3 \varrho}{M}\right)^{1 / 2}, \quad \beta M \ll 1
\end{array}
$$

and the corresponding charge densities of particles in the ground state

$$
\begin{aligned}
& \varrho_{0}=\varrho\left[1-\left(T / T_{c}\right)^{3 / 2}\right], \quad \quad \beta M \gg 1, \\
& \varrho_{0}=\varrho\left[1-\left(T / T_{c}\right)^{2}\right], \quad \quad \beta M \ll 1 .
\end{aligned}
$$


It has to bo noted that for massless bosons, the condition $|\mu| \leq M$ does not require $\mu=0$ like in the flat space, because $M>0$ also for massless particles. This implies that the critical temperature is always finite and so, unlike in the flat case, $\varrho$ is always different from $\varrho_{0}$. As has been noticed in Ref. 266] that on a flat manifold the net charge of massless bosons resides in the Bose-Einstein condensed ground state. This never happens if the spatial manifold is $H^{3}$. In fact, because of constant curvature massive and massless bosons have a similar behaviour.

As it is well known, at the critical temperature, continuous thermodynamic quantities may have a discontinuous derivative (first order phase transition). This stems from the fact that the second derivative with respect to $T$ of the chemical potential is a discontinuos function for $T=T_{c}$. This implies that the first derivative of the specific heat $C_{V}$ has a jump for $T=T_{c}$ given by

$$
\left.\frac{d C_{V}}{d T}\right|_{T_{c}^{+}}-\left.\frac{d C_{V}}{d T}\right|_{T_{c}^{-}}=\Omega T_{c}^{+} \mu^{\prime \prime}\left(\left.T_{c}^{)} \frac{\partial \varrho(T, \mu)}{\partial T}\right|_{T=T_{c}^{+}}\right.
$$

where by the prime we indicate the total derivative with respect to $T$.

Now we again consider the low and high temperature limits and compute the discontinuity of the derivative of the specific heat using Eq. (7.17). The charge density as given in Eq. (7.11) can be obtained by deriving $-\Omega_{\beta}(\beta, \mu) / \Omega$ with respect to $\mu$. For $T>T_{c}$ this gives $\mu$ as an implicit function of $T$ and $\varrho$.

Deriving Eq. (7.8) with respect to $\mu$ and taking only leading terms into account (of course disregarding topological contributions), we get an expansion for $\varrho$ valid for small $T$. It reads

$$
\varrho \simeq\left(\frac{M T}{2 \pi}\right)^{3 / 2} \sum_{n=1}^{\infty} \frac{e^{-n \beta(M-|\mu|)}}{n^{3 / 2}} .
$$

Of course, for $|\mu|=M$ this gives Eq. (7.13). From Eq. (7.18) we obtain

$$
\mu^{\prime \prime}\left(T_{c}^{+}\right) \sim-\frac{2.44}{T_{c}}, \quad T \frac{\partial \varrho}{\partial T}=\frac{3 \varrho}{2}
$$

and the standard result

$$
\left.\frac{d C_{V}}{d T}\right|_{T_{c}^{+}}-\left.\frac{d C_{V}}{d T}\right|_{T_{c}^{-}}=-\frac{3.66 Q}{T_{c}}
$$

immediately follows $(Q=\varrho \Omega$ is the total charge).

In a similar way, deriving Eq. (7.10) with respect to $\mu$ (always disregarding topological contributions), we get the expansion for the charge density

$$
\begin{aligned}
\varrho \simeq & \frac{\mu T^{2}}{3}-\frac{\mu T\left(M^{2}-\mu^{2}\right)^{1 / 2}}{2 \pi}+\frac{\mu\left(3 M^{2}-2 \mu^{2}\right)}{12 \pi^{2}} \\
& +\sum_{k=1}^{\infty} \beta^{2 k} \zeta_{R}^{\prime}(-2 k) \sum_{n=1}^{k} \frac{\mu^{2 n-1}}{(2 n-1) !(k-n) !} \frac{M^{4}}{16 \pi^{2}(k-n+2) !}\left(\frac{M}{2}\right)^{2(k-n)},
\end{aligned}
$$

valid for $T>T c$. For $\mu=M$, the leading term of the latter expression gives again the result (7.14). From Eq. (7.21), by a straightforward computation and taking only leading terms into account we get

$$
\begin{aligned}
& \mu^{\prime \prime}\left(T_{c}^{+}\right) \simeq-\frac{16 \pi^{2}}{9 M}, \\
& \left.\frac{d C_{V}}{d T}\right|_{T_{c}^{+}}-\left.\frac{d C_{V}}{d T}\right|_{T_{c}^{-}} \simeq-\left.\frac{\partial 2 Q \pi^{2}}{9 M}\right|_{T=T_{c}^{+}} \simeq 2 \varrho,
\end{aligned}
$$


in agreement with the result on $\mathbb{R}^{4}$ given in Ref. [266].

We have shown that both massless and massive scalar fields on the Lobachevsky space $H^{3}$ exhibits Bose-Einstein condensation at a critical temperature depending on the curvature of the space. The higher is the curvature radius the higher is the critical temperature. The treatment is not intended to be complete in any sense but we have not been able to display a curvature effect on thermodynamic quantities at the most elementary level. In particular, due to curvature, massless charged bosons have a finite $T_{c}$ in contrast with the flat space result. The difference can be traced back to the existence of a gap in the spectrum of the Laplace operator on $H^{3}$. We also pointed out that the infinite volume limit is under good control only for Dirichlet boundary conditions, for which the smallest eigenvalue has its limit value precisely at the gap of the continuous spectrum.

\subsection{The finite temperature effective potential for a self-interacting scalar field on $\mathbb{R} \times H^{3} / \Gamma$}

As a last example, we shall consider finite temperature effects associated with a self-interacting scalar field on ultrastatic space-time of the form $\mathbb{R} \times H^{3} / \Gamma$. We shall give low and high temperature expansions and we shall obtain the one-loop, finite temperature effective potential as a by-product of free energy.

To start with, let $\phi$ a neutral scalar field non-minimally coupled to the gravitational field and with a self-interacting term of the kind $\lambda \phi^{4}$. We concentrate on the temperature dependent part of the one loop effective potential, the zero temperature contributions, which require renormalization, being computed in Sec. 4.4.2. We shall also assume the isometry group to contain only hyperbolic elements.

After the compactification in the imaginary time $\tau$, the classical action for such a system reads

$$
S_{c}[\phi, g]=\int_{0}^{\beta} d \tau \int_{H^{3} / \Gamma}\left[-\frac{1}{2} \phi\left(\frac{\partial^{2}}{\partial \tau^{2}}+\Delta_{3}\right)+V(\phi, R)\right]|g|^{\frac{1}{2}} d^{3} x
$$

and the small disturbance operator $A=-\partial_{\tau}^{2}+\mathrm{七}_{3}$, with $\mathrm{七}_{3}=-\Delta_{3}+V^{\prime \prime}\left(\phi_{c}, R\right)$. As usual $\phi_{c}$ represents a classical solution around which we expand the action. The potential is assumed to be of the form

$$
V(\phi, R)=\frac{m^{2} \phi^{2}}{2}+\frac{\xi R \phi^{2}}{2}+\frac{\lambda \phi^{4}}{24}
$$

which for $\phi=\phi_{c}$ becomes the classical potential $V_{c}\left(\phi_{c}, R\right)$. From now on we shall leave understood the explicit dependence on $R$.

Assuming a constant background field $\phi_{c}$ the concept of one-loop, finite temperature effective potential is well defined [140] and given by the one-loop free energy density. As in Sec. 2.4 we formally have $V_{\text {eff }}\left(\phi_{c}\right)=V_{c}\left(\phi_{c}\right)+V^{(1)}\left(\phi_{c}\right)$, with the one-loop quantum corrections

$$
V^{(1)}\left(\phi_{c}\right)=\frac{1}{2 \beta \Omega(\mathcal{F})} \ln \operatorname{det}\left(A \ell^{2}\right)=V_{0}^{(1)}\left(\phi_{c}\right)+V_{\beta}^{(1)}\left(\phi_{c}\right)
$$

where we have separated the zero $\left(V_{0}^{(1)}\left(\phi_{c}\right)\right)$ and finite $\left(V_{\beta}^{(1)}\left(\phi_{c}\right)\right)$ temperature contributions.

Using Eqs. (2.63) and (7.6) we directly have

$$
\begin{aligned}
V_{\beta}^{(1)}\left(\phi_{c}\right)= & \frac{1}{\beta \Omega(\mathcal{F})} \operatorname{Tr} \ln \left(1-e^{-\beta Q}\right) \\
= & -\frac{M^{4}}{2 \pi^{2}} \sum_{n=1}^{\infty} \frac{K_{2}(n \beta M)}{(n \beta M)^{2}} \\
& +\frac{1}{\pi \Omega(\mathcal{F})} \sum_{n=1}^{\infty} \int_{0}^{\infty} \cos n \beta t \ln Z\left(1+\sqrt{\left[t^{2}+M^{2}\right] /|\kappa|}\right) d t,
\end{aligned}
$$


where $Q=\left|L_{3}\right|^{1 / 2}$ and

$$
M^{2}=V^{\prime \prime}\left(\phi_{c}\right)+\kappa \varrho_{3}^{2}=m^{2}+\left(\xi-\frac{1}{6} R\right)+\frac{\lambda \phi_{c}^{2}}{2} .
$$

Of course, other representations can be derived from the results concerning the thermodynamic potential in the limit $\mu \rightarrow 0$. In particular we write down the low and high temperature expansions, which we need in the following. They read respectively

$$
\begin{aligned}
V_{\beta}^{(1)}\left(\phi_{c}\right) \sim & -\frac{1}{2 \Omega(\mathcal{F})} \frac{M^{2}}{(2 \pi)^{1 / 2}} \sum_{n=1}^{\infty} \sum_{\{\gamma\}} \sum_{k=1}^{\infty} \frac{\chi^{k}(\gamma) l_{\gamma} e^{-n \beta M \sqrt{1+\left(k l_{\gamma} / n \beta\right)^{2}}}}{S_{3}\left(k ; l_{\gamma}\right)\left(n \beta M \sqrt{1+\left(k l_{\gamma} / n \beta\right)^{2}}\right)^{3 / 2}} \\
& \times\left[1+\frac{3}{4\left(n \beta M \sqrt{1+\left(k l_{\gamma} / n \beta\right)^{2}}\right)}\right]-\frac{1}{2} \frac{M^{4}}{(2 \pi)^{3 / 2}} \sum_{n=1}^{\infty} \frac{e^{-n \beta M}}{(n \beta M)^{5 / 2}} . \\
V_{\beta}^{(1)}(\hat{\phi}) \sim & -\frac{\pi^{2}}{90 \beta^{4}}+\frac{M^{2}}{24 \beta^{2}}-\left[\frac{M^{3}}{12 \pi}-\frac{M}{2 \Omega(\mathcal{F})} \ln Z(1+M)\right] \frac{1}{\beta} \\
& -\frac{M^{4}}{32 \pi^{2}}\left[\ln \frac{\beta M}{4 \pi}+\gamma-\frac{3}{4}\right] \\
& -\frac{M^{2}}{2 \pi \Omega(\mathcal{F})|\kappa|^{1 / 2}} \int_{1}^{\infty} \Xi\left(1+t M|\kappa|^{-1 / 2}\right) \sqrt{t^{2}-1} d t+O\left(\beta^{2}\right),
\end{aligned}
$$

where only leading terms have been written. Of course, also in this case the coefficients of the positive powers of $\beta$ do not depend on the topology. It is interesting to observe that the topological term independent of $\beta$ in the latter formula is the same, but the sign, as the topological contribution to the zero temperature effective potential, also after renormalization (see Eq. (4.17) in Sec. 4.4.2 and Ref. [286]). This means that in the high temperature expansion of the one-loop effective potential only the term proportional to $T=1 / \beta$ feels the non trivial topology (hyperbolic elements only).

Phase transitions. The relevant quantity for analyzing the phase transitions of the system is the mass of the field. The quantum corrections to the mass are defined by means of equation

$$
V_{e f f}\left(\phi_{c}\right)=\Lambda_{e f f}+\frac{1}{2}\left(m_{0}^{2}+m_{\beta}^{2}\right) \phi_{c}^{2}+O\left(\phi_{c}^{4}\right)
$$

where $\Lambda_{\text {eff }}$ (the cosmological constant) in general represents a complicated expression not depending on the background field $\phi_{c}$ while $m_{0}$ and $m_{\beta}$ represent the zero and finite temperature quantum corrections to the mass $m$. As has been shown in Sec. 4.4.2 (see also Ref. 273]), $m_{0}$ has curvature and topological contributions, which help to break symmetry (for $\xi<1 / 6$ ). On the contrary, here we shall see that $m_{\beta}$ always helps to restore the symmetry.

By evaluating the second derivative with respect to $\phi_{c}$ at the point $\phi_{c}=0$ of Eqs. (7.24) and (7.25), we obtain for $m_{\beta}$ the two equations

$$
\begin{aligned}
m_{\beta}^{2}= & \frac{\lambda}{2 \Omega(\mathcal{F})} \operatorname{Tr} \frac{e^{-\beta Q_{0}}}{Q_{0}\left(1-e^{\left.-\beta Q_{0}\right)}\right.} \\
= & \frac{\lambda M_{0}^{2}}{4 \pi^{2}} \sum_{n=1}^{\infty} \frac{K_{1}\left(n \beta M_{0}\right)}{n \beta M_{0}} \\
& +\frac{\lambda}{2 \pi \Omega(\mathcal{F})|\kappa|} \sum_{n=1}^{\infty} \int_{0}^{\infty} \cos n \beta t \frac{\Xi\left(1+\sqrt{\left[t^{2}+M_{0}^{2}\right] /|\kappa|}\right)}{\sqrt{\left[t^{2}+M_{0}^{2}\right] /|\kappa|}} d t,
\end{aligned}
$$


where $Q_{0}=Q_{\phi=0}=\left|-\Delta_{3}+m^{2}+\xi R\right|^{1 / 2}$ and $M_{0}=M_{\phi=0}=\left|m^{2}+(\xi-1 / 6) R\right|^{1 / 2}$. From the exact formula $(7.28)$ we see that the finite temperature quantum corrections to the mass are always positive, their strength mainly depending on the smallest eigenvalues of the operator $Q_{0}$. This means that such a contribution always helps to restore the symmetry. The second expression (7.29) gives the mass in terms of geometry and topology of the manifold. In fact, wew recall that the $\Xi$-function is strictly related to the isometry group $\Gamma$, which realizes the non trivial topology of $\mathcal{M}$.

To go further, we compute the corrections to the mass in the high temperature limit. Using Eq. (7.27) we obtain

$$
\begin{aligned}
m_{\beta}^{2} \sim & \frac{\lambda}{24 \beta^{2}}-\frac{3 M_{0} \lambda}{8 \pi \beta} \\
& +\frac{|\kappa|^{-1 / 2}}{4 M_{0}^{2} \Omega(\mathcal{F})}\left[\ln Z\left(1+M_{0}|\kappa|^{-1 / 2}\right)+M_{0}|\kappa|^{-1 / 2} \Xi\left(1+M_{0}|\kappa|^{-1 / 2}\right)\right] \frac{\lambda M_{0}}{\beta},
\end{aligned}
$$

from which we see that if temperature is high enough, quantum corrections always help to restore symmetry. By using Eq. (7.26) one can also compute the quantum corrections to the mass in the low temperature limit. Of course one obtains exponentially damped corrections dominated by the topological part 272]. 


\section{Strings at finite temperature}

Since the early days of dual string models, it was been known that an essential ingredient of string theory at non-zero temperature was the so-called Hagedorn temperature [287, 288, 226]. It was soon recognized that the appearance of the Hagedorn temperature was a consequence of the fact that the asymptotic form of the state level density had an exponential dependence on the mass. A naive argument led to the conclusion that above such a temperature the free energy was diverging. After these pioneering works, the success of string theory yielded a lot of investigations on finite temperature effects for these extended objects [289, 290, 291, 292], [293, 294, 295, 296], 297, 298, 299]. In Refs. [300, 301] the possible occurrence of a first order transition above the Hagedorn temperature has been discussed. Further recent references are 302, 303, 304, 305. One of the main reasons for these investigations is connected with the thermodynamic of the early universe (see Refs. [306, 307] and references therein) as well as with the attempts to use extended objects for the description of the high temperature limit of the confining phase of large N-SU(N) Yang-Mills theory [308, 309].

We shall try to investigate the finite temperature effects in string theory within the canonical ensemble approach. The existence of the Hagedorn spectrum (the exponentially growing density of states) leads to the breakdown of the correspondence between canonical and microcanonical ensemble above the Hagedorn temperature. Thus our considerations are only valid below this critical temperature. We also mention that the microcanonical ensemble, in some sense more appropriate for the study of strings at finite temperature, has been advocated and used in Ref. 227].

In this section we put the Regge slope parameter $\alpha=1 / 2$, thus the string tension is normalized at $T=1 / \pi$.

\subsection{The Mellin-Barnes representation for one-loop string free energy}

We start by recalling that there are different representations for the string free energy. One of these, which is very useful for formal manipulations, gives a modular invariant expression for the free energy [294, 295, 310]. However, this and all other well known representations (see for example Ref. [305]) are integral ones, in which the Hagedorn temperature appears as the convergence condition in the ultraviolet limit of a certain integral. These may be called propertime representations. In order to discuss high- or low-temperature limits in such representations one has to expand the integral in terms of a corresponding series. Thus, a specific series expansion appears in string theory at non-zero temperature. Here, making use of the so-called Mellin-Barnes representation, we shall exhibit a Laurent representation for the one-loop open (super)string free energy [311, 312].

We have shown how to arrive at Mellin-Barnes representation for field theory free energy at finite temperature in Sec. 2.6. Recalling that the $\zeta$-function density related to the operator $L_{N}=-\Delta_{N}+m^{2}=\vec{p}^{2}+m^{2}$ acting on functions in $\mathbb{R}^{N}$ reads

$$
\tilde{\zeta}\left(s \mid L_{N}\right)=\frac{\Gamma\left(s-\frac{N}{2}\right) m^{N-2 s}}{(4 \pi)^{\frac{N}{2}} \Gamma(s)}
$$

and making use of Eq. (2.61), for the statistical sum of a free massive field in a $D=N+1$ dimensional, flat space-time one has ( $b$ stands for bosons and $f$ for fermions)

$$
\tilde{F}_{\beta}^{b, f}=-\frac{(4 \pi)^{-\frac{D}{2}}}{4 \pi i} \int_{c-i \infty}^{c+i \infty}\left(\frac{\beta}{2}\right)^{-s} \Gamma\left(\frac{s}{2}\right) \Gamma\left(\frac{s-D}{2}\right) \zeta_{b, f}(s) m^{D-s} d s .
$$

Here $\zeta_{b}(s)=\zeta_{R}(s), \zeta_{f}(s)=\left(1-2^{1-s}\right) \zeta_{R}(s)$ and the mass $m$ is a c-number. In order to generalize the above representation to (super)strings, it is sufficient to note that in that case one has to 
deal with a mass operator $M^{2}$. As a consequence, for (super)string theory one can generalize the representation (8.1) in the form

$$
\begin{aligned}
& \mathcal{F}_{\text {string }}=-\frac{(4 \pi)^{-\frac{D}{2}}}{4 \pi i} \int_{c-i \infty}^{c+i \infty}\left(\frac{\beta}{2}\right)^{-s} \Gamma\left(\frac{s}{2}\right) \Gamma\left(\frac{s-D}{2}\right) \zeta_{R}(s) \operatorname{Tr}\left[M^{2}\right]^{\frac{D-s}{2}} d s \\
& \mathcal{F}_{\text {superstring }}=-\frac{(4 \pi)^{-\frac{D}{2}}}{2 \pi i} \int_{c-i \infty}^{c+i \infty}\left(\frac{\beta}{2}\right)^{-s} \Gamma\left(\frac{s}{2}\right) \Gamma\left(\frac{s-D}{2}\right)\left(1-2^{-s}\right) \zeta_{R}(s) \operatorname{Tr}\left[M^{2}\right]^{\frac{D-s}{2}} d s
\end{aligned}
$$

where $D=26$ for strings and $D=10$ for superstrings. The symbol Tr means trace over boson and fermion fields. The quantity $\operatorname{Tr}\left[M^{2}\right]^{(D-s) / 2}$ which appears in above equations requires a regularization because a naive definition of it leads to a formal divergent expression. For this reason we make use of the Mellin transform

$$
\operatorname{Tr}\left[M^{2}\right]^{-s}=\frac{1}{\Gamma(s)} \int_{0}^{\infty} t^{s-1} \operatorname{Tr} e^{-t M^{2}} d t
$$

and the heat-kernel expansion of $\operatorname{Tr} e^{-t M^{2}}$.

For bosonic strings the mass operator contains both infrared (due to the presence of the tachyon in the spectrum) and ultraviolet divergences, while for superstrings it contains only ultraviolet divergences. Furthermore, for closed (super)strings the constraints should be introduced via the usual identity (see for example Ref. 305]). Hence the consideration of open superstrings is simpler from a technical point of view and in the following we shall consider them in some detail.

A simple and standard way to arrive at the heat-kernel expansion is the following. For open superstrings (without gauge group) the mass operator is given by (see for example Ref. [4])

$$
M^{2}=2 \sum_{i=1}^{D-2} \sum_{n=1}^{\infty} n\left(N_{n i}^{b}+N_{n i}^{f}\right), \quad D=10 .
$$

This leads to [四]

$$
\operatorname{Tr} e^{-t M^{2}}=8 \prod_{n=1}^{\infty}\left(\frac{1-e^{-2 t n}}{1+e^{-2 t n}}\right)^{-8}=8\left[\theta_{4}\left(0 \mid e^{-2 t}\right)\right]^{-8},
$$

where $\theta_{4}(x, y)$ is the Jacobi elliptic theta-function (see Appendix $D$ for definition and properties) and the presence of the factor 8 is due to the degeneracy of the ground states.

Using Eq. (D.8) or alternatively Meinardus theorem in Eq. (8.3) we obtain the asymptotics for small $t$

$$
\left[\theta_{4}\left(0, e^{-2 t}\right)\right]^{-8} \sim \frac{t^{4}}{(2 \pi)^{4}} e^{\pi^{2} / t}-\frac{t^{4}}{2 \pi^{4}}+O\left(e^{-\pi^{2} / t}\right) .
$$

So we may define the regularized trace of the complex power $\operatorname{Tr}\left(M^{2}\right)^{\frac{D-s}{2}}(D=10)$ in the following way

$$
\begin{aligned}
\operatorname{Tr}\left(M^{2}\right)^{5-\frac{s}{2}}=\frac{8}{\Gamma\left(\frac{s}{2}-5\right)} \int_{0}^{\infty} t^{\frac{s}{2}-6} & {\left[\left[\theta_{4}\left(0, e^{-2 t}\right)\right]^{-8}-\frac{t^{4}}{(2 \pi)^{4}}\left(e^{\pi^{2} / t}-8\right)\right] d t } \\
& +\frac{8}{(2 \pi)^{4} \Gamma\left(\frac{s}{2}-5\right)} \int_{0}^{\infty} t^{\frac{s}{2}-2}\left(e^{2 \pi^{2} / t}-8\right) d t,
\end{aligned}
$$

where the latter integral has to be understood in the sense of analytical continuation. This means that $\int_{0}^{\infty} t^{s / 2-2} d t=0$, while

$$
\int_{0}^{\infty} t^{\frac{s}{2}-2} e^{\pi^{2} / t}=\left(-\pi^{2}\right)^{\frac{s}{2}-1} \Gamma\left(1-\frac{s}{2}\right) .
$$


The final result then assumes the form

$$
\operatorname{Tr}\left(M^{2}\right)^{5-\frac{s}{2}}=\frac{1}{2 \pi^{6} \Gamma\left(\frac{s}{2}-5\right)}\left[(-1)^{\frac{s}{2}-1} \pi^{s} \Gamma\left(1-\frac{s}{2}\right)+\pi^{3 / 2} G(s, \Lambda)\right],
$$

where we have set

$$
G(s, \Lambda)=\sqrt{\pi}\left(\frac{\pi}{2}\right)^{\frac{s}{2}-1} \int_{0}^{\Lambda} t^{\frac{s}{2}-6}\left\{\left[\frac{1}{2} \theta_{4}\left(0, e^{-\pi t}\right)\right]^{-8}-t^{4}\left(e^{2 \pi / t}-8\right)\right\} d t .
$$

In Eq. (8.5) the infrared cutoff parameter $\Lambda$ has been introduced. On the next stage of our calculations this regularization will be removed (i.e. we will take $\Lambda \rightarrow \infty$ ).

Making use of Eqs. (8.2) and (8.4) we get

$$
\begin{aligned}
& \mathcal{F}_{\text {superstring }}=-\frac{(2 \pi)^{-11}}{2 \pi i} \int_{c-i \infty}^{c+i \infty}[\varphi(s)+\psi(s)] d s, \\
& \varphi(s)=(-1)^{\frac{s}{2}-1}\left(1-2^{-s}\right) \zeta_{R}(s) \frac{\pi}{\sin \frac{\pi s}{2}}\left(\frac{\beta}{2 \pi}\right)^{-s}, \\
& \psi(s)=\left(1-2^{-s}\right) \pi^{\frac{3}{2}-s} \zeta_{R}(s) \Gamma\left(\frac{s}{2}\right) G(s, \Lambda)\left(\frac{\beta}{2 \pi}\right)^{-s} .
\end{aligned}
$$

The meromorphic function $\varphi(s)$ has first order poles at $s=1$ and $s=2 k, k=1,2, \ldots$ The pole of $\varphi$ at $s=1$ has imaginary residue. The meromorphic function $\psi(s)$ has first order poles at $s=1$. One can see that the regularization cutoff parameter can be removed. If $c>1$, closing the contour in the right half-plane, we obtain

$$
\begin{aligned}
\mathcal{F}_{\text {superstring }}= & -\frac{2}{(2 \pi)^{11}}\left[\sum_{k=1}^{\infty}\left(1-2^{-2 k}\right) \zeta_{R}(2 k) x^{2 k}-\frac{\pi x}{4} G(1, \infty)\right] \\
& +I_{R}(x)+I_{R}(x, \Lambda),
\end{aligned}
$$

where $x=\beta_{c} / \beta, \beta_{c}=2 \pi$, while $I_{R}(x)$ and $I_{R}(x, \Lambda)$ are the contributions coming from the contour integrals of the functions $\phi(s)$ and $\varphi(s)$ respectively along the arc of radius $R$ in the right half-plane. The series converges when $\beta>\beta_{c}=2 \pi, \beta_{c}$ being the Hagedorn temperature (see for example Ref. [297]). The sum of the series can be explicitly evaluated and the result is

$$
\sum_{k=1}^{\infty}\left(1-2^{-2 k}\right) \zeta_{R}(2 k) x^{2 k}=\frac{\pi x}{4} \tan \frac{\pi x}{2}, \quad|x|<1 .
$$

As a consequence the statistical sum contribution to the one-loop free energy is given by

$$
\mathcal{F}_{\text {superstring }}=-\frac{\pi x}{2(2 \pi)^{11}}\left[\tan \frac{\pi x}{2}-G(1, \infty)\right]+I_{R}(x)+I_{R}(x, \Lambda) .
$$

If $|x|<1$ then the value of the contour integral $I_{R}(x)$ is vanishing when $R \rightarrow \infty$. With regard to the contour contribution $I_{R}(x, \Lambda)$, we observe that we can remove the cut-off $\Lambda$ and it has the corrected low temperature limit (see for example Ref. [228]). For high temperature it is negligible.

We conclude by observing that for the open bosonic string one can repeat all the steps and arrive at the series representation

$$
\sum_{k=1}^{\infty} \zeta_{R}(2 k) y^{2 k}=\frac{1}{2}-\frac{\pi y}{2} \cot (\pi y), \quad|y|<1 .
$$


As a result, for $y<1$ one can show that the high temperature expansion assumes the form

$$
\mathcal{F}_{\text {bosonic string }} \simeq-\frac{1}{2^{23} \pi^{16}}\left[-y \cot (\pi y)-\frac{2 y}{\beta_{c}} D(1, \Lambda)+\frac{1}{\pi} D(0, \Lambda)\right] .
$$

Here $y=\beta_{c} / \beta$, the related Hagedorn temperature is $\beta_{c}=\sqrt{8} \pi$,

$$
D(s, \Lambda)=2 \pi \int_{0}^{\Lambda} t^{\frac{s}{2}-14}\left[\eta(i t)^{-24}-t^{12} e^{2 \pi / t}\right] d t
$$

and finally $\eta(\tau)=e^{i \pi \tau / 12} \prod_{n=1}^{\infty}\left(1-e^{2 \pi i n \tau}\right)$ is the Dedekind eta function. However in this case, the infrared cutoff parameter $\Lambda$ cannot be removed for the presence of a tachyon in the spectrum. We observe that a similar asymptotic behaviour was already been pointed out in Ref. [226], but in a different context.

The Laurent series have been obtained for $|x|<1$ and $|y|<1$, namely for $\beta>\beta_{c}$. The right hand sides of the above formulae, Eqs. (8.8) and (8.9), may be understood as an analytic continuations of those series for $|x|>1,|y|>1$ (i.e. $\beta<\beta_{c}$ ). As a consequence we have exhibited a kind of periodic structure for the one-loop free energy of (super)strings.

The results we have obtained here are based on the Mellin-Barnes representation for the one-loop free energy of the critical (super)strings. Such a novel representation for the lowest order in string perturbation theory has permitted to obtain explicit thermodynamic expressions in term of a Laurent series. The critical temperature arises in this formalism as the convergence condition (namely the radius of convergence) of these series. Furthermore, the explicit analytic continuation of the free energy for temperatures beyond the critical Hagedorn one $\left(\beta \leq \beta_{c}\right)$ has been found. As a result, there might be the possibility to analyze the breakdown of the canonical ensemble and possible new string phases.

It is somewhat surprising that exists such a finite temperature periodic structure in the behaviour of (super)string thermodynamic quantities. The typical widths of the periodic sectors depends on the Regge slope parameter $\alpha$. The widths of the sectors grow together with the parameter $\alpha$ and in the limit $\alpha \rightarrow 0$ (string tension goes to $\infty$ ), the thermodynamic system can be associated with ideal massless gas of quantum fields present in the normal modes of the string (see for example Ref. [289]). In addition, from Eq. (8.6) it follows that $\mathcal{F}_{\text {superstring }} \sim \beta^{-10}$ and such behaviour is consistent with the ordinary statistical mechanics results (see for example Ref. [228]).

\subsection{High genus contributions to string free energy}

The physical meaning of the Hagedorn temperature as the critical one corresponding to the behaviour of thermodynamic ensembles, may also be grasped by investigating the interplay between free strings and their interactions (i.e. higher loops). In this subsection we would like to generalize to arbitrary genus- $g$ strings the Mellin-Barnes representation previously obtained. Such a generalization will allow us to identify the critical temperature at arbitrary loop order. Early attempts to study the critical temperature for multi-loop strings has been presented in Refs. 313, 314, 315. Here we closely follow Ref. 316].

It is well-known that the genus- $g$ temperature contribution to the free energy for the bosonic string can be written as 313

$$
F_{g}(\beta)=\sum_{m_{i}, n_{j}=-\infty}^{\infty} \int(d \tau)_{W P}\left(\operatorname{det} P^{\dagger} P\right)^{1 / 2}\left(\operatorname{det} \Delta_{g}\right)^{-13} e^{-\Delta S(\beta, \vec{m}, \vec{n})},
$$

where $(d \tau)_{W P}$ is the Weil-Petersson measure on the Teichmüller space. This measure as well as the factors $\operatorname{det}\left(P^{\dagger} P\right)$ and $\operatorname{det} \Delta_{g}$ are each individually modular invariant [313]. In addition

$$
I_{g}(\tau)=\left(\operatorname{det} P^{\dagger} P\right)^{1 / 2}\left(\operatorname{det} \Delta_{g}\right)^{-13}=e^{C(2 g-2)} Z^{\prime}(1)^{-13} Z(2),
$$


where $Z(s)$ is the Selberg zeta function and $C$ an universal constant [317]. Furthermore, the winding-number factor has the form of a metric over the space of windings, namely

$$
\Delta S(\beta, \vec{m}, \vec{n})=\frac{T \beta^{2}}{2}\left[m_{l} \Omega_{l i}-n_{i}\right]\left[(\operatorname{Im} \Omega)^{-1}\right]_{i j}\left[\bar{\Omega}_{j k} m_{k}-n_{j}\right]=g^{\mu \nu}(\Omega) N_{\mu} N_{\nu}
$$

$T$ being the string tension (which here is explicitly written) and $\mu, \nu=1,2, \ldots, 2 g,\left\{N_{1}, \ldots, N_{2 g}\right\} \equiv$ $\left\{m_{1}, n_{1}, \ldots, m_{g}, n_{g}\right\}$. The periodic matrix $\Omega$ corresponding to the string world-sheet of genus $g$ is a holomorphic function of the moduli, $\Omega_{i j}=\Omega_{j i}$ and $\operatorname{Im} \Omega>0$. The matrix $\Omega$ admits a decomposition into real symmetric $g \times g$ matrices, that is $\Omega=\Omega_{1}+i \Omega_{2}$. As a result

$$
g\left(\Omega_{1}+i \Omega_{2}\right)=\left(\begin{array}{cc}
\Omega_{1} \Omega_{2}^{-1} \Omega_{1}+\Omega_{2} & -\Omega_{1} \Omega_{2}^{-1} \\
-\Omega_{2}^{-1} \Omega_{1} & \Omega_{2}^{-1}
\end{array}\right) .
$$

Besides, $g(\Omega)=\hat{\Lambda}^{t} g(\Lambda(\Omega)) \hat{\Lambda}$ [317], where $\Lambda$ is an element of the symplectic modular group $S p(2 g, \mathbb{Z})$ and the associated transformation of the periodic matrix reads $\Omega \mapsto \Omega^{\prime}=\Lambda(\Omega)=$ $(A \Omega+B)(C \Omega+D)^{-1}$. As a consequence, the winding factor

$$
\sum_{\vec{m}, \vec{n}}^{\prime} \exp [-\Delta S(\beta, \vec{m}, \vec{n})]
$$

is also modular invariant.

It can be shown that the $2 g$ summations present in the expression for $F_{g}(\beta)$ can be replaced by a single summation together with a change in the region of integration from the fundamental domain to the analogue of the strip $S_{a_{1}}$ related to the cycle $a_{1}$, whose choice is entirely arbitrary [313]. Then, one has

$$
F_{g}(\beta)=\sum_{r=1}^{\infty} \int(d \tau)_{W P} I_{g}(\tau) \exp \left[-\frac{T \beta^{2} r^{2}}{2} \Omega_{1 i}\left[(\operatorname{Im} \Omega)^{-1}\right]_{i j} \bar{\Omega}_{j 1}\right] .
$$

Let us now consider the Mellin-Barnes representation for the genus- $g$ free energy. A simple way to arrive at it is to make use of the Mellin transform of the exponential factor, i.e.

$$
e^{-v}=\frac{1}{2 \pi i} \int_{c-i \infty}^{c+i \infty} \Gamma(s) v^{-s} d s
$$

with $\operatorname{Re} v>0$ and $c>0$. Therefore one gets

$$
\begin{aligned}
\sum_{\vec{m}, \vec{n}}{ }^{\prime} \exp [-\Delta S(\beta, \vec{m}, \vec{n})] & =\sum_{\vec{m}, \vec{n}}{ }^{\prime} \frac{1}{2 \pi i} \int_{c-i \infty}^{c+i \infty} \Gamma(s)(\Delta S(\beta, \vec{m}, \vec{n}))^{-s} d s \\
& =\frac{1}{2 \pi i} \int_{c-i \infty}^{c+i \infty} \Gamma(s)\left(\frac{\beta^{2}}{2 \pi}\right)^{-s} G_{g}(s ; \Omega) d s
\end{aligned}
$$

where

$$
\begin{aligned}
& G_{g}(s ; \Omega) \equiv \sum_{\vec{N} \in \mathbb{Z}^{2 g} /\{0\}}\left(\vec{N}^{t} \Omega \vec{N}\right)^{-s}, \\
& \sum_{r=1}^{\infty} \exp \left[-\frac{\beta^{2} r^{2}}{2 \pi} \Omega_{1 i}\left[(\operatorname{Im} \Omega)^{-1}\right]_{i j} \bar{\Omega}_{j 1}\right]= \\
& \\
& \quad \frac{1}{2 \pi i} \int_{c-i \infty}^{c+i \infty} d s \Gamma(s) \zeta_{R}(2 s)\left(\frac{\beta^{2}}{2 \pi}\right)^{-s}\left\{\Omega_{1 i}\left[(\operatorname{Im} \Omega)^{-1}\right]_{i j} \bar{\Omega}_{j 1}\right\}^{-s} .
\end{aligned}
$$


Finally, using the formulae (8.13) and 8.14) in Eqs. (8.10) and 8.12) respectively, one obtains

$$
\begin{aligned}
& F_{g}(\beta)=\frac{1}{2 \pi i} \int_{c-i \infty}^{c+i \infty} d s \Gamma(s)\left(\frac{\beta^{2}}{2 \pi}\right)^{-s}\left\{\int d(\tau)_{W P} I_{g}(\tau) G_{g}(s ; \Omega)\right\}_{(\operatorname{Reg})}, \\
& F_{g}(\beta)=\frac{1}{2 \pi i} \int_{c-i \infty}^{c+i \infty} d s \Gamma(s) \zeta_{R}(2 s)\left(\frac{\beta^{2}}{2 \pi}\right)^{-s} \\
& \times\left\{\int(d \tau)_{W P} I_{g}(\tau)\left[\Omega_{1 i}\left[(\operatorname{Im} \Omega)^{-1}\right]_{i j} \bar{\Omega}_{j 1}\right]^{-s}\right\}_{(\operatorname{Reg})} .
\end{aligned}
$$

These are the main formulae which will be used for the evaluation of the genus- $g$ string contribution. In order to deal with such expressions, the integrals on a suitable variable in $(d \tau)_{W P}$ should be understood as the regularized ones. In this way the order of integration may be interchanged.

For the sake of simplicity let us reconsider briefly the $g=1$ case. It is well known that 317]

$$
(d \tau)_{W P}=\frac{d \tau_{1} d \tau_{2}}{2 \tau_{2}^{2}}, \quad I_{1}(\tau)=-\frac{\operatorname{Vol}\left(\mathbb{R}^{26}\right)}{(2 \pi)^{13}}\left[\tau_{2}|\eta(\tau)|^{4}\right]^{-12},
$$

where $\eta(\tau)$ is Dedekind eta function defined above and $\operatorname{Vol}\left(\mathbb{R}^{26}\right)$ is the volume of a large region in $\mathbb{R}^{26}$. In the case of an open bosonic string we have $\Omega_{1}=0, \Omega_{2}=\tau_{2}$ and $\Omega=\operatorname{diag}\left(\tau_{2}, \tau_{2}^{-1}\right)$. In the limit $\tau_{2} \rightarrow 0$ we get

$$
\begin{aligned}
& \exp \left[-\frac{\beta^{2}}{2 \pi}\left(\vec{N}^{t} \Omega \vec{N}\right)^{-s}\right] \mapsto \exp \left[-\frac{\beta^{2}}{2 \pi} n^{-2 s} \tau_{2}^{-1}\right], \\
& G_{1}(s ; \Omega) \mapsto \sum_{n=1}^{\infty}\left(n^{2} \tau_{2}^{-1}\right)^{-s}=\tau_{2}^{s} \zeta_{R}(2 s) .
\end{aligned}
$$

The corresponding contribution to free energy is given by

$$
\frac{1}{2 \pi i} \int_{c-i \infty}^{c+i \infty} d s \Gamma(s)\left(\frac{\beta^{2}}{2 \pi}\right)^{-s} \zeta_{R}(2 s)\left\{\int_{0}^{\infty} d \tau_{2} \tau_{2}^{s-14} \eta\left(i \tau_{2}\right)^{-24}\right\}_{(R e g)} .
$$

After having regularized the ultraviolet region $\left(\tau_{2} \rightarrow 0\right)$ one has again Eq. (8.9) of previous Subsection.

By analogy with the above one-loop evaluation, now we shall consider the open string genus$g$ contribution to the free energy. The matrix $\Omega$ may be chosen as $\Omega=\operatorname{diag}\left(\Omega_{2}, \Omega_{2}^{-1}\right)$. In the limit $\Omega_{2} \rightarrow 0$ one has

$$
\begin{aligned}
& \exp \left[-\frac{\beta^{2}}{2 \pi}\left(\vec{N}^{t} \Omega \vec{N}\right)^{-s}\right] \mapsto \exp \left[-\frac{\beta^{2}}{2 \pi} \Omega_{2}^{-1} \vec{N}^{t} \vec{N}\right], \\
& G_{g}(s ; \Omega) \mapsto \Omega_{2}^{s} \sum_{\vec{N} \in \mathbb{Z}^{g} /\{0\}}\left(\vec{N}^{t} \vec{N}\right)^{-s}=\Omega_{2}^{s} Z_{I_{g}}\left(\frac{2 s}{g} ; 0,0\right),
\end{aligned}
$$

where $Z$ is the Epstein zeta function (see Appendix $\mathrm{D}$ for definition and properties) and $I$ the $g \times g$ identity matrix. In this way the contribution to free energy reads

$$
\frac{1}{2 \pi i} \int_{c-i \infty}^{c+i \infty} d s \Gamma(s)\left(\frac{\beta^{2}}{2 \pi}\right)^{-s} Z_{I_{g}}\left(\frac{2 s}{g} ; 0,0\right)\left\{\int d \tau_{W P} \Omega_{2}^{s} I_{g}(\tau)\right\}_{(\operatorname{Reg})} .
$$


Since a tachyon is present in the spectrum, the total free energy will be divergent for any $g$. The infrared divergence may be regularized by means of a suitable cutoff parameter. Such a kind of behaviour can be associated with the procedure of pinching a cycle non homologous at zero (see for example Ref. 315]). It is well known that the behaviour of the factor $(d \tau)_{W P} I_{g}(\tau)$ is given by the Belavin-Knizhnik double-pole result and it has a universal character for any $g$. It should also be noticed that this divergence is $\beta$-independent and the meromorphic structure is similar to the genus-one case. As a consequence, the contribution to the free energy relative to high temperature, may be obtained again in terms of a Laurent-like series and the whole genus dependence of the critical temperature is encoded in the analogue of Riemann zeta-function, namely Epstein zeta function. For this reason we have to determine the asymptotic properties of $Z$. To this aim we make use of the following general result:

$$
C_{g} \equiv \lim _{\operatorname{Re} s \rightarrow \infty} \frac{Z_{I_{g}}\left(\frac{2(s+1)}{g} ; \vec{b}, 0\right)}{Z_{I_{g}}\left(\frac{2 s}{g} ; \vec{b}, 0\right)}=\left[\left(\hat{b}_{1}-\eta_{1}\right)^{2}+\cdots+\left(\hat{b}_{g}-\eta_{g}\right)^{2}\right]^{-1},
$$

where at least one of the $b_{i}$ is noninteger. By $\hat{b}_{i}=b_{i}-\left[b_{i}\right]<1$ we indicate the decimal part of $b_{i}$, while $\eta_{i}$ is equal to 0 or 1 according to whether $\hat{b}_{i} \leq 1 / 2$ or $\hat{b}_{i} \geq 1 / 2$. Furthermore, if $\vec{b}=(0,0, \ldots, 0)$ then $C_{g}=1$. This is just our case. Then we arrive at the conclusion that the interactions of bosonic strings do not modify the critical Hagedorn temperature in full agreement with other computations 313, 315.

One can consider also different linear real bundles over compact Riemann surfaces and spinorial structures on them. The procedure of evaluation of the free energy in terms of the path integral over the metrics $g_{\mu \nu}$ does not depend on whatever type of real scalars are considered. This fact leads to new contributions to the genus- $g$ integrals (8.10) and Eq. (8.11). On the other hand, one can investigate the role of these contributions for the torus compactification [317, 318]. In this case, the sum in Eq. (8.10) should be taken over the vectors on the lattice on which some space dimensions are compactified. The half-lattice vectors can be labelled by the multiplets $\left(b_{1}, . ., b_{p}\right)$, with $b_{i}=1 / 2$. The critical temperature related to the multiplet $\vec{b}=\left(b_{1}, \ldots b_{p}, 0, \ldots, 0\right)$ can be easily evaluated by means of Eq. (8.16), which gives $C_{p}=4 p^{-1}$. As a result

$$
T_{c, p}=\frac{\sqrt{p}}{2} T_{c}
$$

We note that the three particular multiplets in which only one or two or three components of $\vec{b}$ are equal to $1 / 2$ and all the others are vanishing are associated with "minimal" critical temperatures given by $T_{c, 1}=T_{c} / 2, T_{c, 2}=T_{c} / \sqrt{2}$ and $T_{c, 3}=T_{c} / \sqrt{3}$ respectively.

\section{Acknowledgments}

We would like to thank Alfred A. Actor, Roberto Camporesi, Emilio Elizalde, Klaus Kirsten and Sergei D. Odintsov for useful discussions and suggestions. A.A. Bytsenko thanks the Istituto Nazionale di Fisica Nucleare and the Dipartimento di Fisica dell'Università di Trento for financial support and Prof. Marco Toller for the kind hospitality at the Theoretical Group of the Department of Physics of the Trento University. 


\section{Appendices}

\section{A Admissible regularization functions for the determinant}

For the sake of completeness we give in this appendix a list of admissible regularization functions for the logarithm of the determinant discussed in Sec. 2.3, which are often used in the literature (see also Ref. [42]). We limit our analysis to the physically interesting case $N=4$. The $N$ dimensional case can be treated along the same line.

1. Let us start with

$$
\varrho_{1}(\varepsilon, t)=\frac{d}{d \varepsilon} \frac{t^{\varepsilon}}{\Gamma(\varepsilon)}=\frac{t^{\varepsilon}}{\Gamma(\varepsilon)}\{\ln t-\psi(\varepsilon)\},
$$

where $\psi(\varepsilon)$ is the logarithmic derivative of $\Gamma(\varepsilon)$. This is the zeta-function regularization introduced in Refs. [22] and popularized in the physical literature in the seminal paper [8]. All requirements are satisfied. The related $B_{e}(\varepsilon, y)$ and $V(\varepsilon, f)$, say $B_{1}(\varepsilon, y)$ and $V_{1}(\varepsilon, f)$ read

$$
\begin{aligned}
B_{1}(\varepsilon, y) & =y^{-\varepsilon} \ln y=\ln y+O(\varepsilon) \\
V_{1}(\varepsilon) & =-\frac{\mu^{4} y^{2}}{32 \pi^{2}} \frac{d}{d \varepsilon} \frac{\Gamma(\varepsilon-2) y^{-\varepsilon}}{\Gamma(\varepsilon)}=\frac{\mu^{4} y^{2-\varepsilon}}{64 \pi^{2}} \frac{\Gamma(\varepsilon-2)}{\Gamma(\varepsilon)}[\ln y-\psi(\varepsilon-2)+\psi(\varepsilon)] \\
& =\frac{M^{4}}{64 \pi^{2}}\left[\ln \frac{M^{2}}{\mu^{2}}-\frac{3}{2}\right]+O(\varepsilon) .
\end{aligned}
$$

We see that the effective potential is finite, the divergent terms being removed by the particular structure of $\varrho_{1}$. This is the regularization we have been used throughout the paper.

2. The next regularization we shall consider is closely related to the above one, and in some sense is associated with the familiar dimensional regularization in momentum space. It is defined by 94

$$
\varrho_{2}(\varepsilon, t)=t^{\varepsilon} .
$$

From this,

$$
\begin{aligned}
B_{2}(\varepsilon, y) & =-y^{-\varepsilon} \Gamma(\varepsilon)=\ln y+\gamma-\frac{1}{\varepsilon}+O(\varepsilon), \\
V_{2}(\varepsilon) & =-\frac{\mu^{4} y^{2-\varepsilon}}{32 \pi^{2}} \Gamma(\varepsilon-2) \\
& =\frac{M^{4}}{64 \pi^{2}}\left[\ln \frac{M^{2}}{\mu^{2}}-\frac{3}{2}+\gamma\right]-\frac{M^{4}}{64 \pi^{2} \varepsilon}+O(\varepsilon),
\end{aligned}
$$

easily follow, again in agreement with the general result. Within this regularization, only one divergent term is present. 
3. Another often used regularization is the ultraviolet cut-off regularization, defined by

$$
\varrho_{3}(\varepsilon, t)=\theta(t-\varepsilon) .
$$

In this case we have

$$
\begin{aligned}
B_{3}(\varepsilon, y) & =-\Gamma(0, y \varepsilon)=\ln y+\gamma+\ln \varepsilon+O(\varepsilon) \\
V_{3}(\varepsilon) & =-\frac{\mu^{4} y^{2} \Gamma(-2, \varepsilon y)}{32 \pi^{2}} \\
& =\frac{M^{4}}{64 \pi^{2}}\left[\ln \frac{M^{2}}{\mu^{2}}-\frac{3}{2}+\gamma\right]-\frac{\mu^{4}}{64 \pi^{2} \varepsilon^{2}}+\frac{\mu^{2} M^{2}}{32 \pi^{2} \varepsilon}+\frac{M^{4}}{64 \pi^{2}} \ln \varepsilon+O(\varepsilon) .
\end{aligned}
$$

Here we have three divergent terms. From these examples, it is obvious that they depend on the regularization function.

4. The fourth regularization reads

$$
\varrho_{4}(\varepsilon, t)=e^{-\varepsilon / 4 t} \text {. }
$$

We have

$$
\begin{aligned}
B_{4}(\varepsilon, y) & =-2 K_{0}(\sqrt{\varepsilon y})=\ln y+2 \gamma-2 \ln 2+\ln \varepsilon+O(\varepsilon) \\
V_{4}(\varepsilon) & =-\frac{\mu^{4} y}{4 \varepsilon \pi^{2}} K_{2}(\sqrt{\varepsilon y}) \\
& =\frac{M^{4}}{64 \pi^{2}}\left[\ln \frac{M^{2}}{\mu^{2}}-\frac{3}{2}+2 \gamma-2 \ln 2\right]-\frac{\mu^{4}}{2 \pi^{2} \varepsilon^{2}}+\frac{\mu^{2} M^{2}}{8 \pi^{2} \varepsilon}+\frac{M^{4}}{64 \pi^{2}} \ln \varepsilon+O(\varepsilon),
\end{aligned}
$$

$K_{\nu}$ being the Mc Donald functions.

5. The last regularization we would like to consider is presented as an example of the freedom one has. It is similar to a Pauli-Villars type and it is defined by ( $\alpha$ being an arbitrary positive constant)

$$
\varrho_{5}(\varepsilon, t)=\left(1-e^{-\alpha t / \varepsilon}\right)^{3},
$$

the power 3 being related to the fact that we are working in four dimensions. This is a general feature of the Pauli-Villars regularization. We have

$$
\begin{aligned}
& B_{5}(\varepsilon, y)= \ln \frac{\varepsilon y}{\varepsilon y+3 \alpha}+3 \ln \frac{\varepsilon y+2 \alpha}{\varepsilon y+\alpha}=\ln y+\ln \frac{8}{3 \alpha}+\ln \varepsilon+O(\varepsilon) \\
& V_{5}(\varepsilon)=\frac{\mu^{4} y^{2}}{64 \pi^{2}}\left\{\left[\ln \frac{\varepsilon y}{\alpha}-\frac{3}{2}\right]-3\left(1+\frac{\alpha}{\varepsilon y}\right)^{2}\left[\ln \left(1+\frac{\varepsilon y}{\alpha}\right)-\frac{3}{2}\right]\right. \\
&\left.\quad+3\left(1+\frac{2 \alpha}{\varepsilon y}\right)^{2}\left[\ln \left(2+\frac{\varepsilon y}{\alpha}\right)-\frac{3}{2}\right]-\left(1+\frac{3 \alpha}{\varepsilon y}\right)^{2}\left[\ln \left(3+\frac{\varepsilon y}{\alpha}\right)-\frac{3}{2}\right]\right\} \\
&=\frac{M^{4}}{64 \pi^{2}}\left[\ln \frac{M^{2}}{\mu^{2}}-\frac{3}{2}+\ln \frac{8 \alpha}{3}\right]+\frac{3 \alpha^{2} \ln (16 / 27) \mu^{4}}{64 \pi^{2} \varepsilon^{2}} \\
&+\frac{3 \alpha \ln (16 / 9) \mu^{2} M^{2}}{64 \pi^{2} \varepsilon}+\frac{M^{4}}{64 \pi^{2}} \ln \varepsilon+O(\varepsilon) .
\end{aligned}
$$

With this example we conclude the list of possible regularization functions. 


\section{B The heat kernel on a Riemannian manifold without boundary}

\section{B.1 Spectral coefficients for a Laplace-like operator}

In the paper we need some coefficients of the heat kernel expansion for a second order elliptic differential operator $A=-\Delta+V(x)$ acting on neutral scalar fields on Riemannian manifold without boundary. In this case some coefficients have been computed by many authors [32, 27]. They are given by

$$
\begin{aligned}
& a_{0}(x)=1, \quad a_{1}(x)=-V(x)+\frac{1}{6} R(x), \\
& a_{2}(x)=\frac{1}{2}\left(a_{1}\right)^{2}+\frac{1}{6} \Delta a_{1}(x)+\frac{1}{180}\left(\Delta R+R^{i j r s} R_{i j r s}-R^{i j} R_{i j}\right), \\
& a_{3}(x)=\frac{1}{6}\left(a_{1}\right)^{3}+a_{1}\left[a_{2}-\frac{1}{2}\left(a_{1}\right)^{2}\right] \\
& +\frac{1}{12} V^{; i} V_{; i}-\frac{1}{60} \Delta^{2} V+\frac{1}{90} R^{i j} V_{; i j}-\frac{1}{30} R^{; i} V_{; i} \\
& +\frac{1}{7 !}\left\{18 \Delta^{2} R+17 R_{; i} R^{; i}-2 R_{i j ; r} R^{i j ; r}-4 R_{i j ; r} R^{i r ; j}\right. \\
& +9 R_{i j r s ; q} R^{i j r s ; q}-8 R_{i j} \Delta R^{i j}+24 R_{i j} R_{r}^{i r ; j}+12 R_{i j r s} \Delta R^{i j r s} \\
& -\frac{208}{9} R_{i j} R^{i r} R_{r}^{j}+\frac{64}{3} R_{i j} R_{r s} R^{i r j s}-\frac{16}{3} R_{i j} R_{r s q}^{i} R^{j r s q} \\
& \left.+\frac{44}{9} R_{i j r s} R^{i j m n} R_{m n}^{r s}+\frac{80}{9} R_{i j r s} R^{i m r n} R_{m n}^{j}{ }_{n}\right\} .
\end{aligned}
$$

We refer the interested reader to Ref. [37] for the spectral coefficients on a Riemannian manifold with boundary and to Ref. [38] for the spectral coefficients on a Riemann-Cartan manifold.

In some physical problems it may be convenient to factorize the exponential $\exp \left(t a_{1}\right)$ and consider an expansion (as introduced in Refs. [10, 11]) very closely related to Eq. (2.12), that is

$$
K_{t}^{0}(x, x) \sim \frac{e^{t a_{1}}}{(4 \pi t)^{N / 2}} \sum_{n=0}^{\infty} b_{n}(x) t^{n},
$$

with $b_{0}=1, b_{1}=0$ and more generally

$$
b_{n}(x)=\sum_{l=0}^{n} \frac{(-1)^{l} a_{n-l} a_{1}^{l}}{l !}, \quad a_{n}(x)=\sum_{l=0}^{n} \frac{b_{n-l} a_{1}^{l}}{l !} .
$$

In this way, as proved in Ref. [12], all coefficients $b_{n}$ do not depend explicitly on $V(x)$, but eventually on its derivatives. The coefficients $b_{n}$ up to $b_{3}$ have been computed in Ref. [12] and read

$$
\begin{aligned}
& b_{2}(x)=\frac{1}{36} \Delta R-\frac{1}{6} \Delta V+\frac{1}{180}\left(\Delta R+R_{i j r s} R^{i j r s}-R_{i j} R^{i j}\right), \\
& b_{3}(x)=\frac{1}{12} V^{; i} V_{; i}-\frac{1}{60} \Delta^{2} V+\frac{1}{90} R^{i j} V_{; i j}-\frac{1}{30} R^{; i} V_{; i} \\
& +\frac{1}{7 !}\left\{18 \Delta^{2} R+17 R_{; i} R^{; i}-2 R_{i j ; r} R^{i j ; r}-4 R_{i j ; r} R^{i r ; j}\right. \\
& +9 R_{i j r s ; q} R^{i j r s ; q}-8 R_{i j} \Delta R^{i j}+24 R_{i j} R^{i r ; j}{ }_{r}+12 R_{i j r s} \Delta R^{i j r s} \\
& -\frac{208}{9} R_{i j} R^{i r} R_{r}^{j}+\frac{64}{3} R_{i j} R_{r s} R^{i r j s}-\frac{16}{3} R_{i j} R_{r s q}^{i} R^{j r s q} \\
& \left.+\frac{44}{9} R_{i j r s} R^{i j m n} R_{m n}^{r s}+\frac{80}{9} R_{i j r s} R^{i m r n} R_{m n}^{j}{ }_{n}\right\} \text {. }
\end{aligned}
$$




\section{B.2 Heat kernel exact solutions on constant curvature manifolds}

Here we derive recurrence relations for the heat kernel related to the Laplacian of functions on spheres and hyperbolic manifolds. Then we just write down some known results concerning $S^{N}$, $H^{N}$ and $T^{N}$. Other exact solutions on homogeneous spaces and bibliography on this subject can be found for example in Ref. [6].

The recurrence relation. As simple examples of manifolds in which the heat kernel related to the Laplacian $\Delta_{N}$ on functions can be exactly computed and given in a closed form, here we consider Riemannian manifolds with constant curvature. We use normal coordinates $y^{k}\left(x, x^{\prime}\right)$ about the point $x$ and indicate by $\sigma$ the geodesic distance between $x$ and $x^{\prime}$. Because of homogeneity, we expect the heat kernel $K_{t}\left(x, x^{\prime} \mid-\Delta_{N}\right)$ to depend only upon the geodesic distance between $x$ and $x^{\prime}$. Then, choosing a scalar density $\tilde{f}(\sigma)$ of weight $-1 / 2$ depending only upon $\sigma$, a direct computation shows (note that $\tilde{f}(\sigma)=\left[g_{N}(\sigma)\right]^{1 / 4} f(\sigma), f(\sigma)$ being a true scalar and $g_{N}(\sigma)$ the determinant of the metric tensor)

$$
\Delta_{N} \tilde{f}(\sigma)=\tilde{f}^{\prime \prime}(\sigma)+\frac{N-1}{\sigma} \tilde{f}^{\prime}(\sigma)-\left(\beta_{N}^{\prime}+\beta^{2} N+\frac{N-1}{\sigma} \beta_{N}\right) \tilde{f}(\sigma),
$$

where by the prime we indicate the derivative with respect to $\sigma$ and $\beta_{N}(\sigma)=\partial_{\sigma} \ln \left[g_{N}(\sigma)\right]^{1 / 4}$. For $g_{N}(\sigma)$ one has

$$
\begin{array}{ll}
g_{N}(\sigma)=\left(\frac{\sin \sigma}{\sigma}\right)^{2(N-1)}, & \text { for } S^{N}, \\
g_{N}(\sigma)=\left(\frac{\sinh \sigma}{\sigma}\right)^{2(N-1)}, & \text { for } H^{N} .
\end{array}
$$

For convenience and without loss of generality, we have normalized the constant curvature $\kappa$ to 1 for the sphere $S^{N}$ and to -1 for the hyperbolic space $H^{N}$. In this way $\sigma$ is dimensionless. The curvature can be restored in all the formulae by simple dimensional arguments.

It is known (unpublished result of Millson reported in Ref. [114], proved in Ref. [6]), that the heat kernel $K_{t}\left(\sigma \mid-\Delta_{N+2}\right)$ on a $N+2$ dimensional space of constant curvature can be obtained from $K_{t}\left(\sigma \mid-\Delta_{N}\right)$ by applying what is called "intertwining operator" in Ref. [6]. In our notation and taking into account that we are working with scalar densities, such an operator is given by

$$
D_{N}=-\frac{1}{2 \pi} g_{N}(\sigma)^{1 / 4} \frac{\partial}{\sigma \partial \sigma} g_{N}(\sigma)^{-1 / 4}=-\frac{1}{2 \pi \sigma}\left(\partial_{\sigma}-\beta_{N}(\sigma)\right) .
$$

The following properties of $D_{N}$ can be directly proved:

$$
\begin{aligned}
& D_{N}\left[\Delta_{N}+\kappa N\right] \tilde{f}(\sigma)=\Delta_{N+2} D_{N} \tilde{f}(\sigma), \\
& D_{N}\left[g_{N}(\sigma)\right]^{1 / 4} \delta_{N}=\left[g_{N+2}(\sigma)\right]^{1 / 4} \delta_{N+2},
\end{aligned}
$$

where $\delta_{N}$ is the Dirac delta function on $S^{N}$ or $H^{N}$. From Eq. (B.8) we then obtain

$$
\begin{aligned}
& \left(\partial_{t}-\Delta_{N+2}-\kappa N\right) D_{N} K_{t}\left(\sigma \mid-\Delta_{N}\right)=0, \\
& \lim _{t \rightarrow 0_{+}} D_{N} K_{t}\left(x, x^{\prime} \mid-\Delta_{N}\right)=\left[g_{N+2}(x)\right]^{1 / 4} \delta_{N+2}\left(x, x^{\prime}\right)\left[g_{N+2}\left(x^{\prime}\right)\right]^{1 / 4},
\end{aligned}
$$

from which directly follows

$$
K_{t}\left(\sigma \mid-\Delta_{N+2}\right)=e^{t \kappa N} D_{N} K_{t}\left(\sigma \mid-\Delta_{N}\right),
$$

where $N \geq 1$ for $S^{N}(\kappa=1)$ and $N \geq 2$ for $H^{N}(\kappa=-1)$. The integral version of such recurrence relation, Eq. (B.12), was obtained in Secs. 3.2 and 3.4.5. Iterating this equation 
we obtain the heat kernel for the Laplacian on $S^{N}$ by knowing the kernels $K_{t}^{S^{1}}(\sigma)$ or $K_{t}^{S^{2}}(\sigma)$ according to whether $N$ is odd or even. In a similar way we get the kernel on $H^{N}$ starting from $K_{t}^{H^{2}}(\sigma)$ or $K_{t}^{H^{3}}(\sigma)$.

It has been shown in Ref. [6] that $K_{t}\left(\sigma \mid-\Delta_{N}\right)$ can be related to $K_{t}\left(\sigma \mid-\Delta_{N-1}\right)$ by introducing fractional derivatives of semi-integer order. In this manner, the knowledge of $K_{t}^{S^{1}}(\sigma)$ or $K_{t}^{H^{2}}(\sigma)$ is sufficient in order to get the heat kernel in any dimensional smooth Riemannian space of constant curvature.

The torus case. Because of flatness, the solution of the heat equation for the Laplacian on $S^{1}=T^{1}$ can be easily derived from the solution on $\mathbb{R}$, making use of the method of images. The heat kernel on $\mathbb{R}^{N}$ is well known to be

$$
K_{t}^{\mathbb{R}^{N}}\left(x, x^{\prime}\right)=\frac{1}{(4 \pi t)^{N / 2}} e^{-\left(x-x^{\prime}\right)^{2} / 4 t} .
$$

Putting $N=1$ and replacing $\left(x-x^{\prime}\right)^{2}$ with $(\sigma+2 \pi n r)^{2}(r>0$ being the radius of curvature of $S^{1}$ ), we obtain an expression which of course satisfy the heat equation on $S^{1}$. By summing over all $n$, we have

$$
K_{t}^{S^{1}}(\sigma)=\frac{1}{\sqrt{4 \pi t}} \sum_{n=-\infty}^{\infty} e^{-(\sigma+2 \pi n r)^{2} / 4 t}
$$

The heat kernel for the Laplacian on a $N$-dimensional torus can be directly obtained by observing that $T^{N}=S^{1} \times \ldots \times S^{1}$ is the direct product of $N$ circles. Then using Eqs. (B.14) and (2.11) one gets

$$
K_{t}^{T^{N}}(\vec{\sigma})=\frac{1}{(4 \pi t)^{N / 2}} \sum_{\vec{k}} e^{-(\vec{\sigma}+2 \pi \vec{k}) \cdot \mathcal{R}(\vec{\sigma}+2 \pi \vec{k}) / 4 t},
$$

where $\vec{k}, \vec{\sigma} \in \mathbb{Z}^{N}$ and $\mathcal{R}$ is the diagonal matrix $\mathcal{R}=\operatorname{diag}\left(r_{1}^{2}, \ldots, r_{N}^{2}\right), r_{i}$ being the radii of the $N$ circles $S^{1}$.

The sphere case. Looking at Eq. (B.12), it follows that the solution on the odd dimensional spheres looks quite similar to Eq. (B.14). For example, for $N=3$ we immediately get

$$
K_{t}^{S^{3}}(\sigma)=\frac{e^{t a^{2}}}{(4 \pi t)^{3 / 2}} \sum_{n=-\infty}^{\infty}\left(1+\frac{2 \pi n / a}{\sigma}\right) e^{-(\sigma+2 \pi n / a)^{2} / 4 t},
$$

where $a^{2}=\kappa$ has been put. Note that this expression looks different from the one given, for example, in Ref. [6], because we are working with scalar densities.

The solution on $S^{2}$ looks very differently and can be expressed for example in terms of Legendre polynomials. It has also the integral representation [6]

$$
K_{t}^{S^{2}}(\sigma)=\frac{\sqrt{2} e^{t a^{2} / 4}}{(4 \pi t)^{3 / 2}}\left(\frac{\sin a \sigma}{a \sigma}\right)^{1 / 2} \sum_{n=-\infty}^{\infty}(-1)^{n} \int_{\sigma}^{\pi / a} \frac{\left(a \sigma^{\prime}+2 \pi n\right) e^{-\left(\sigma^{\prime}+2 \pi n / a\right)^{2} / 4 t}}{\left(\cos a \sigma-\cos a \sigma^{\prime}\right)^{1 / 2}} d \sigma^{\prime} .
$$

The hyperbolic case. The solution on $H^{N}$ can be derived from the corresponding solution on $S^{N}$, noting that the passage from $S^{N}$ to $H^{N}$ is formally given by the replacement $|\kappa| \rightarrow-|\kappa|$, that is $a \rightarrow i a(a=\sqrt{|\kappa|})$. Of course, due to the non compactness of $H^{N}$, one has to take into account only the "direct path" $n=0$. Then, from Eqs. (B.16) and (B.17) for $H^{3}$ and $H^{2}$ we get respectively

$$
K_{t}^{H^{3}}(\sigma)=\frac{e^{-t a^{2}} e^{-\sigma^{2} / 4 t}}{(4 \pi t)^{3 / 2}}
$$




$$
K_{t}^{H^{2}}(\sigma)=\frac{\sqrt{2} e^{-a^{2} t / 4}}{(4 \pi t)^{3 / 2}}\left(\frac{\sinh a \sigma}{a \sigma}\right)^{1 / 2} \int_{\sigma}^{\infty} \frac{a \sigma^{\prime} e^{-\sigma^{\prime 2} / 4 t}}{\left(\cosh a \sigma^{\prime}-\cosh a \sigma\right)^{1 / 2}} d \sigma^{\prime}
$$

\section{The explicit computation of $\zeta$-function on compact manifolds without boundary}

Here we collect the explicit representations for $\zeta$-function on several manifolds which are used in the text. The operator is assumed to be of the form $\mathrm{七}_{N}=-\Delta_{N}+\alpha^{2}+\kappa \varrho_{N}^{2}$, with $\varrho_{N}=(N-1) / 2$ and $\alpha$ an arbitrary constant.

Example: $S^{1}$. Using Eq. (3.9) and Poisson summation formula (D.1) (or alternatively Eq. (B.14)) in Eq. (2.13) we get

$$
\begin{aligned}
\zeta\left(s \mid \mathrm{E}_{1}\right)-\frac{\Omega_{1} \Gamma(s-1 / 2) \alpha^{1-2 s}}{\sqrt{4 \pi} \Gamma(s)} & =\frac{2 \Omega_{1} \alpha^{1-2 s}}{\sqrt{\pi} \Gamma(s)} \sum_{n=1}^{\infty} \frac{K_{1 / 2-s}(2 \pi n \alpha)}{(\pi n \alpha)^{1 / 2-s}} \\
& =\frac{2 \Omega_{1} \alpha^{1-2 s} \sin \pi s}{\pi} \int_{1}^{\infty} \frac{\left(u^{2}-1\right)^{-s}}{e^{2 \pi \alpha u}-1} d u,
\end{aligned}
$$

from which we directly read off the residues of the poles of $\zeta$-function at the points $s=1 / 2-k$ $(k=0,1, \ldots)$. As for the torus, the representation of $\zeta$ in terms of Mc Donald functions is valid for any $s$, while the last integral is convergent only for $\operatorname{Re} s<1$, but this is sufficient for our aims. We incidentally note that the last term in Eq. (C.2) looks quite similar to the contribution

of hyperbolic elements on a compact hyperbolic manifold (see Eq. (3.53)). The right hand sides of the above formula, in the limit $\alpha \rightarrow 0$, give the $\zeta$-function in the massless case.

Example: $S^{3}$. Using Eqs. (3.15) (C.1) and (C.2) we obtain

$$
\begin{aligned}
\zeta\left(s \mid \mathrm{E}_{3}\right)-\frac{\Omega_{3} \Gamma(s-3 / 2) \alpha^{3-2 s}}{(4 \pi)^{3 / 2} \Gamma(s)} & \\
=\frac{\Omega_{3} \alpha^{3-2 s}}{(\pi)^{3 / 2} \Gamma(s)} \sum_{n=1}^{\infty}[ & {\left[\frac{(s-1) K_{3 / 2-s}(2 \pi n \alpha)}{(\pi n \alpha)^{3 / 2-s}}-\frac{K_{1 / 2-s}(2 \pi n \alpha)}{(\pi n \alpha)^{1 / 2-s}}\right] } \\
& =-\frac{\Omega_{3} \alpha^{3-2 s} \sin \pi s}{\pi^{2}} \int_{1}^{\infty} \frac{u^{2}\left(u^{2}-1\right)^{-s}}{e^{2 \pi \alpha u}-1} d u .
\end{aligned}
$$

A similar representation in terms of Mc Donald functions has been derived in Refs. [99, 100].

Example: $S^{2}$. Choosing the path of integration $z=r e^{ \pm \pi / 2}$ in Eq. (3.18) we get

$$
\zeta\left(s \mid \mathrm{E}_{2}\right)=\frac{\Omega_{2}}{4(s-1)}\left[\int_{0}^{\alpha} \frac{\left(\alpha^{2}-r^{2}\right)^{-(s-1)}}{\cosh ^{2} \pi r} d r-\cos \pi s \int_{\alpha}^{\infty} \frac{\left(r^{2}-\alpha^{2}\right)^{-(s-1)}}{\cosh ^{2} \pi r} d r\right],
$$

which is valid for $\operatorname{Re} s<2$ and $\alpha>0$.

Example: $H^{3} / \Gamma$. Here we only report identity and elliptic contributions to heat kernel and $\zeta$-function, hyperbolic contributions being expressed in terms of Selberg $Z$-function for any $N$ (see Sec. 3.4.5). We have

$$
K_{I}\left(t \mid \mathrm{七}_{3}\right)=\frac{\Omega\left(\mathcal{F}_{3}\right) e^{-t \alpha^{2}}}{(4 \pi t)^{3 / 2}},
$$




$$
\begin{aligned}
\zeta_{I}\left(s \mid \mathrm{E}_{3}\right) & =\frac{\Omega\left(\mathcal{F}_{3}\right) \Gamma\left(s-\frac{3}{2}\right)}{(4 \pi)^{\frac{3}{2}} \Gamma(s)} \alpha^{3-2 s}, \\
\zeta_{I}^{\prime}\left(0 \mid \mathrm{E}_{3}\right) & =\frac{\Omega\left(\mathcal{F}_{3}\right) \alpha}{6 \pi} \\
K_{E}\left(t \mid \mathrm{E}_{3}\right) & =\frac{E e^{-t \alpha^{2}}}{\sqrt{4 \pi t}} \\
\zeta_{E}\left(s \mid \mathrm{E}_{3}\right) & =\frac{E \Gamma\left(s-\frac{1}{2}\right)}{\sqrt{4 \pi} \Gamma(s)} \alpha^{1-2 s}, \\
\zeta_{E}^{\prime}\left(0 \mid \mathrm{E}_{3}\right) & =-E \alpha .
\end{aligned}
$$

Example: $H^{2} / \Gamma$. Again, identity and elliptic contributions read

$$
\begin{aligned}
K_{I}\left(t \mid \mathrm{E}_{2}\right) & =\frac{\Omega\left(\mathcal{F}_{2}\right) e^{-t \alpha^{2}}}{4 \pi t} \int_{0}^{\infty} \frac{\pi e^{-t r^{2}}}{\cosh ^{2} \pi r} d r \\
& =\frac{\Omega\left(\mathcal{F}_{2}\right) e^{-t \alpha^{2}}}{4 \pi t} \sum_{n=0}^{\infty} \frac{B_{2 n}}{n !}\left(2^{1-2 n}-1\right) t^{n}, \\
\zeta_{I}\left(s \mid \mathrm{E}_{2}\right) & =\Omega\left(\mathcal{F}_{2}\right)\left[\frac{\alpha^{2-2 s}}{4 \pi(s-1)}-\frac{1}{\pi} \int_{0}^{\infty} \frac{r\left(r^{2}+\alpha^{2}\right)^{-s}}{1+e^{2 \pi r}} d r\right], \\
\zeta_{I}^{\prime}\left(0 \mid \mathrm{E}_{2}\right) & =\Omega\left(\mathcal{F}_{2}\right)\left[\frac{\alpha^{2}}{4 \pi}\left(\ln \alpha^{2}-1\right)+\frac{1}{\pi} \int_{0}^{\infty} \frac{r \ln \left(r^{2}+\alpha^{2}\right)}{1+e^{2 \pi r}} d r\right], \\
K_{E}\left(t \mid \mathrm{E}_{2}\right) & =\frac{e^{-t \alpha^{2}}}{2 \pi} \int_{-\infty}^{\infty} e^{-t r^{2}} E_{2}(r) d r \\
\zeta_{E}\left(s \mid \mathrm{E}_{2}\right) & =\int_{-\infty}^{\infty}\left(r^{2}+\alpha^{2}\right)^{-s} E_{2}(r) d r \\
\zeta_{E}^{\prime}\left(0 \mid \mathrm{E}_{2}\right) & =-\int_{-\infty}^{\infty} \ln \left(r^{2}+\alpha^{2}\right) E_{2}(r) d r
\end{aligned}
$$

where for convenience we have introduced the function

$$
E_{2}(r)=\sum_{\{\alpha\}} \sum_{j=1}^{m_{\alpha}-1}\left[2 m_{\alpha} \sin \frac{\pi j}{m_{\alpha}}\right]^{-1} \frac{\exp \left(-\frac{2 \pi r j}{m_{\alpha}}\right)}{1+\exp (-2 \pi r)} .
$$

In Eq. (C.9), $B_{n}$ are the Bernoulli numbers and the series is convergent for $0<t<2 \pi$.

Looking at the above equations we see that elliptic elements of isometry group modify the heat coefficients. This is due to the fact that $H^{N} / \Gamma$ is not a smooth manifold when elliptic groups are taken into consideration. In particular for $N=3$, from Eqs. (C.5) and (C.7) we easily obtain

$$
K_{2 n}\left(\mathrm{E}_{3}\right)=\frac{\left(-\alpha^{2}\right)^{n}}{n !}\left[\frac{\Omega(\mathcal{F})}{(4 \pi)^{\frac{3}{2}}}-\frac{4 \pi n E}{(4 \pi)^{\frac{1}{2}} \alpha^{2}}\right]
$$

and of course $K_{2 n+1}\left(\mathrm{E}_{3}\right)=0$ since $H^{3} / \Gamma$ is a manifold without boundary. The residues of $\zeta\left(s \mid \mathrm{E}_{3}\right)$ at the poles $s=\frac{3}{2}-n(n \geq 0)$ immediately follow from Eqs. (2.20) and (C.12). We have

$$
\operatorname{Res}\left(\zeta\left(\frac{3}{2}-n \mid \mathrm{E}_{3}\right)\right)=\frac{\left(-\alpha^{2}\right)^{n}}{\Gamma(n+1) \Gamma\left(\frac{3}{2}-n\right)}\left[\frac{\Omega(\mathcal{F})}{(4 \pi)^{\frac{3}{2}}}-\frac{n E}{(4 \pi)^{\frac{1}{2}} \alpha^{2}}\right] .
$$




\section{Useful relations}

For reader convenience we collect some definitions and properties of special functions which we used throughout the article.

The Poisson summation formula. This is one of the most useful summation formulae. It can be regarded as a non-abelian version of the Selberg trace formula. There exist several versions. To begin with, in the sense of distributions we have

$$
\sum_{n=-\infty}^{\infty} \delta(x-2 \pi n)=\frac{1}{2 \pi} \sum_{n=-\infty}^{\infty} e^{i n x}
$$

In general, for any suitable function $f(\vec{x}), \vec{x} \in \mathbb{R}^{N}$ and any $\vec{q} \in \mathbb{R}^{N}$, the Poisson summation formula reads

$$
\sum_{\vec{k} \in \mathbb{Z}^{N}} f(\vec{k}+\vec{q})=\sum_{\vec{k} \in \mathbb{Z}^{N}} \int_{\mathbb{R}^{N}} f(\vec{x}) e^{2 \pi i \vec{k} \cdot(\vec{x}-\vec{q})} d^{N} x .
$$

The Mellin transform. Let $x^{z-1} f(x)$ belong to $L(0, \infty)$ and let $f(x)$ have bounded variation on every finite interval. Then the Mellin transform is defined by

$$
\hat{f}(z)=\int_{0}^{\infty} x^{z-1} f(x) d x .
$$

In the case when $f(x)$ is continuos, the Mellin inversion formula is

$$
f(x)=\frac{1}{2 \pi i} \int_{\operatorname{Re} z=c} x^{-z} \hat{f}(z) d z
$$

$c$ being a real number in the strip in which $\hat{f}(z)$ is analytic. For any pair of functions $f, g$ with Mellin transforms $\hat{f}, \hat{g}$, we have the useful Mellin-Parseval identity

$$
\int_{0}^{\infty} f(x) g(x) d x=\frac{1}{2 \pi i} \int_{\operatorname{Re} z=c} \hat{f}(z) \hat{g}(1-z) d z
$$

where $c$ is in the common strip in which $\hat{f}(z)$ and $\hat{g}(1-z)$ are analytic.

Mc Donald $K_{\nu}(z)$-functions. The following integral representations [107] are frequently used in the paper:

$$
\begin{aligned}
K_{\nu}(z)= & \frac{\left(\frac{z}{2}\right)^{\nu} \Gamma\left(\frac{1}{2}\right)}{\Gamma\left(\nu+\frac{1}{2}\right)} \int_{1}^{\infty} e^{-z t}\left(t^{2}-1\right)^{\nu-\frac{1}{2}} d t \\
& {\left[\operatorname{Re}\left(\nu+\frac{1}{2}\right)>0 \text { and }|\arg z|<\frac{\pi}{2} \quad \text { or } \operatorname{Re} z=0 \text { and } \nu=0\right], } \\
K_{\nu}(z)= & \frac{1}{2}\left(\frac{z}{2}\right)^{\nu} \int_{0}^{\infty} \frac{e^{-t-\frac{z^{2}}{4 t}}}{t^{\nu+1}} d t \quad\left[|\arg z|<\frac{\pi}{2} \text { and } \operatorname{Re} z^{2}>0\right] .
\end{aligned}
$$

Riemann-Hurwitz functions. The prototype of the zeta-functions is the celebrated RiemannHurwitz $\zeta_{H}$-function. For $\operatorname{Re} s>0$, it may defined by means of equation (see for example [107])

$$
\zeta_{H}(s ; q)=\sum_{n=0}^{\infty}(n+q)^{-s}
$$


the sum being extended to all non-negative $n$ such that $n+q \neq 0$. Here $q$ is an arbitrary real number. We have the simple relation $\zeta_{H}(s ; 1)=\zeta_{R}(s)$, where $\zeta_{R}(s)$ is the usual Riemann function. It satisfies the useful functional equation

$$
\pi^{-\frac{s}{2}} \Gamma\left(\frac{s}{2}\right) \zeta_{R}(s)=\pi^{-\frac{1-s}{2}} \Gamma\left(\frac{1-s}{2}\right) \zeta_{R}(1-s) .
$$

The Epstein $Z$-function. The Epstein $Z$-function can be considered as a generalization of the one of Riemann-Hurwitz. We consider three $N$ dimensional vectors $\vec{q}$ and $\vec{h}$ in $\mathbb{R}^{N}$ and $\vec{k} \in \mathbb{Z}^{N}$ and an invertible $N \times N$ matrix $\mathcal{R}$. For $\operatorname{Re} s>1$ the Epstein Z-function is defined by 319

$$
Z_{\mathcal{R}}(s ; \vec{q}, \vec{h})=\sum_{\vec{k}} e^{2 \pi i \vec{k} \cdot \vec{h}}[(\vec{k}+\vec{q}) \mathcal{R}(\vec{k}+\vec{q})]^{-N s / 2},
$$

where the sum run over $\vec{k} \in \mathbb{Z}^{N}$ for which $\vec{k}+\vec{q} \neq 0$. If all components of $\vec{h}$ are not integer, then Eq. (D.5) can be analytically continued to an entire function in the complex plane, otherwise it has a simple pole at $s=1$ with residue $\pi^{N s / 2} \operatorname{det} \mathcal{R}^{-1 / 2} \Gamma(1+N / 2) . Z_{\mathcal{R}}(s ; \vec{q}, \vec{h})$ satisfies the functional equation

$$
\begin{aligned}
& \pi^{-\frac{N s}{2}} \Gamma\left(\frac{N s}{2}\right) Z_{\mathcal{R}}(s ; \vec{q}, \vec{h})= \\
& \quad \operatorname{det} \mathcal{R}^{-\frac{1}{2}} \pi^{-\frac{N(1-s)}{2}} e^{-2 \pi i \vec{q} \cdot \vec{h}} \Gamma\left(\frac{N(1-s)}{2}\right) Z_{\mathcal{R}^{-1}}(1-s ; \vec{h},-\vec{q}) .
\end{aligned}
$$

The Jacobi elliptic $\theta_{4}$-function. In the paper we shall use just $\theta_{4}(u, q)$, which is defined by (we follow Ref. [107])

$$
\theta_{4}(u, q)=\sum_{n=-\infty}^{\infty}(-1)^{n} q^{n^{2}} e^{2 \pi i u}
$$

and can be represented also as an infinite product. In particular one has

$$
\theta_{4}(0, q)=\prod_{n=1}^{\infty} \frac{1-q^{n}}{1+q^{n}} .
$$

Setting $q=e^{-t}$ and using the dual property

$$
\theta_{4}\left(0, e^{-t}\right)=\sqrt{\frac{\pi}{t}} \sum_{n=-\infty}^{\infty} e^{-\pi^{2}(n-1 / 2)^{2} / t},
$$

we obtain the asymptotic expansion (for small $t$ )

$$
\theta_{4}\left(0, e^{-t}\right) \sim 2 \sqrt{\frac{\pi}{t}}\left[e^{-\frac{\pi^{2}}{4 t}}+e^{-\frac{(3 \pi)^{2}}{4 t}}+e^{-\frac{(5 \pi)^{2}}{4 t}}+\ldots\right] .
$$




\section{References}

[1] B.S. DeWitt. Phys. Rep. 19, 295 (1975).

[2] N.D. Birrell and P.C.W. Davies. Quantum Fields in Curved Space. Cambridge University Press, Cambridge, (1982).

[3] S.A. Fulling. Aspects of Quantum Field Theory in Curved Space-Time. Cambridge University Press, Cambridge, (1989).

[4] M.B. Green, J.H. Schwarz and E. Witten. Superstring Theory. Cambridge University Press, Cambridge, (1987).

[5] A. Selberg. J. Indian Math. Soc. 20, 47 (1956).

[6] R. Camporesi. Phys. Rep. 196, 1 (1990).

[7] G.W. Gibbons. Quantum field theory in curved spacetime. In General Relativity. An Einstein Centenary Survey. S.W. Hawking and W. Israel, editors. Cambridge University Press, Cambridge, (1979).

[8] S.W. Hawking. Commun. Math. Phys. 55, 133 (1977).

[9] L. Parker. Aspects of Quantum Field in Curved Spacetime. In Recent Developments in Gravitation. S. Deser and M. Levy, editors, 219-273. Plenum Publishing Corp., (1979). New York.

[10] L. Parker and D.J. Toms. Phys. Rev. D31, 953 (1985).

[11] L. Parker and D.J. Toms. Phys. Rev. D31, 2424 (1985).

[12] I. Jack and L. Parker. Phys. Rev. D31, 2439 (1985).

[13] E. Elizalde, S. D. Odintsov, A. Romeo, A.A. Bytsenko and S. Zerbini. Zeta Regularization Techniques with Applications. World Scientific, (1994).

[14] R.P. Feynmann and A.R. Hibbs. Quantum Mechanics and Path Integrals. McGraw-Hill, New York, (1965).

[15] L.D. Faddeev and V.N. Popov. Phys. Rev. Lett. 25, 30 (1967).

[16] C. Bernard. Phys. Rev. D9, 3312 (1974).

[17] K. Fujikawa. Phys. Rev. Lett. 44, 1733 (1980).

[18] E.S. Abers and B.W. Lee. Phys. Rep. 9, 1 (1973).

[19] S.W. Hawking. Phys. Rev. D18, 1747 (1978).

[20] S.W. Hawking. The path-integral approach to quantum gravity. In General Relativity. An Einstein Centenary Survey. S.W. Hawking and W. Israel, editors. Cambridge University Press, Cambridge, (1979).

[21] J.D. Bekenstein and L. Parker. Phys. Rev. D23, 2850 (1981).

[22] D.B. Ray and I.M. Singer. Advances in Math. 7, 145 (1971).

[23] D.B. Ray and I.M. Singer. Ann. Math. 98, 154 (1973). 
[24] B.S. DeWitt. Quantum gravity: the new syntesis. In General Relativity. An Einstein Centenary Survey. S.W. Hawking and W. Israel, editors. Cambridge University Press, Cambridge, (1979).

[25] D.J. Toms. Phys. Rev. D35, 3796 (1987).

[26] L. Hormander. Acta Math. 121, 193 (1968).

[27] P.B. Gilkey. J. Diff. Geometry 10, 601 (1975).

[28] G. Cognola, L. Vanzo and S. Zerbini. Phys. Lett. B223, 416 (1989).

[29] R.T. Seeley. Am. Math. Soc. Prog. Pure Math. 10, 172 (1967).

[30] P. Greiner. Arch. Rat. Mech. and Anal. 41, 163 (1971).

[31] S. Minakshisundaram and A. Pleijel. Can. J. Math. 1, 242 (1949).

[32] B.S. DeWitt. The Dynamical Theory of Groups and Fields. Gordon and Breach, New York, (1965).

[33] M. Atiyah R. Bott and V.K. Patodi. Inven. Math. 19, 279 (1973).

[34] V.N. Romanov and A.S. Schwarz. Teor. Mat. Fiz. 41, 190 (1979), (in Russian).

[35] J. Schwinger. Phys. Rev. 82, 664 (1951).

[36] H.P. Mckean Jr. and I.M. Singer. J. Diff. Geom. 1, 43 (1967).

[37] T.P. Branson and P.B. Gilkey. Commun. Partial Differ. Equ. 15, 245 (1990).

[38] G. Cognola and S. Zerbini. Phys. Lett. B214, 70 (1988).

[39] G. Cognola, L. Vanzo and S. Zerbini. Phys. Lett. B241, 381 (1990).

[40] D.M. McAvity and H. Osborn. Class. Quantum Grav. 8, 603 (1991).

[41] S. Minakshisundaram. Can. J. Math. 1, 320 (1949).

[42] R.D. Ball. Phys. Rep. 182, 1 (1989).

[43] S. Coleman and E. Weinberg. Phys. Rev. D7, 1888 (1973).

[44] J. Iliopoulos, C. Itzykson and A. Martin. Rev. Mod. Phys. 47, 165 (1975).

[45] R. Utiyama and B. DeWitt. J. Math. Phys. 3, 608 (1962).

[46] B.L. Nelson and P. Panangaden. Phys. Rev. D25, 1019 (1982).

[47] I.L. Buchbinder, S.D. Odintsov and I.L. Shapiro. Rivista Nuovo Cimento 12, 1 (1989).

[48] I.L. Buchbinder, S.D. Odintsov and I.L. Shapiro. Effective Action in Quantum Gravity. A. Hilger, Bristol, (1992).

[49] D.J. O'Connor, B.L. Hu and T.C. Shen. Phys. Lett. B130, 31 (1983).

[50] B.L. Hu and D.J. O'Connor. Phys. Rev. D30, 743 (1984).

[51] S.W. Hawking and G.F. Ellis. The Large Scale Structure of Space-Time. Cambridge University Press, Cambridge, (1973). 
[52] G.W. Gibbons and M.J. Perry. Proc. R. Soc. Lond A358, 467 (1978).

[53] J.S. Dowker and G. Kennedy. J. Phys. A11, 895 (1978).

[54] D. Page. Phys. Rev. D25, 1499 (1982).

[55] M.R. Brown and A.C. Ottewill. Phys. Rev. D31, 2514 (1985).

[56] M.R. Brown, A.C. Ottewill and D. Page. Phys. Rev. D33, 2840 (1986).

[57] V.P. Gusynin and V.V. Roman'kov. Sov. J. Nucl. Phys. 46, 1097 (1987).

[58] J.S. Dowker and J.P. Schofield. Phys. Rev. D38, 3327 (1988).

[59] J.S. Dowker and J.P. Schofield. Nucl. Phys. B327, 267 (1989).

[60] K. Kirsten. Class. Quantum Grav. 8, 2239 (1991).

[61] R. H. Brandenberger. Rev. Mod. Phys. 57, 1 (1985).

[62] B.L. Hu. Phys. Lett. B108, 19 (1982).

[63] L. Parker and S.A. Fulling. Phys. Rev. D9, 341 (1974).

[64] L. Parker, S.A. Fulling and B.L. Hu. Phys. Rev. D10, 3905 (1974).

[65] B.L. Hu. Phys. Lett. B103, 331 (1981).

[66] B.L. Hu. Phys. Lett. B123, 189 (1983).

[67] P. Anderson and L. Parker. Phys. Rev. D36, 2963 (1987).

[68] A.D. Linde. Phys. Lett. B123, 185 (1983).

[69] B. Allen. Nucl. Phys. B226, 228 (1983).

[70] L. Dolan and R. Jackiw. Phys. Rev. D9, 3320 (1974).

[71] S. Weinberg. Phys. Rev. D9, 3357 (1974).

[72] J.I. Kapusta. Finite-Temperature Field Theory. Cambridge University Press, Cambridge, (1989).

[73] B. Allen. Phys. Rev. D33, 3640 (1986).

[74] A.A. Bytsenko, L. Vanzo and S. Zerbini. Mod. Phys. Lett. A7, 397 (1992).

[75] B. Freedman and L. McLerran. Phys. Rev. D16, 1130 (1977).

[76] J. Kapusta. Phys. Rev. D24, 426 (1981).

[77] A. Actor. Phys. Lett. B157, 53 (1985).

[78] A. Actor. Nucl. Phys. B256, 689 (1986).

[79] G. Cognola and L. Vanzo. Mod. Phys. Lett. A7, 3677 (1992).

[80] H.E. Haber and H.A. Weldon. J. Math. Phys. 23, 1852 (1982).

[81] G. Plunien, B. Müller and W. Greiner. Phys. Rep. 134, 87 (1986). 
[82] T.H. Boyer. Phys. Rev. 174, 1764 (1968).

[83] R. Balian and B. Duplantier. Ann. Phys. 112, 165 (1978).

[84] K.A. Milton. Phys. Rev. D22, 1441 (1980).

[85] T. Kunimasa. Nucl. Phys. B177, 528 (1981).

[86] A.M. Cetto and L. de La Peã. Nuovo Cimento B108, 447 (1993).

[87] B.S. Kay. Phys. Rev. D20, 3052 (1979).

[88] L. Vepstas and A.D. Jackson. Phys. Rep. 187, 109 (1990).

[89] C.M. Bender and P. Hays. Phys. Rev. D14, 2622 (1976).

[90] K. Symanzik. Nucl. Phys. B190, 1 (1981).

[91] S.A. Fulling. Phys. Rev. D7, 2850 (1973).

[92] J. Ambjörn and S. Wolfram. Ann. Phys. 147, 1 (1983).

[93] G. Cognola, L. Vanzo and S. Zerbini. J. Math. Phys. 33, 222 (1992).

[94] J.S. Dowker and R. Critchley. Phys. Rev. D13, 3224 (1976).

[95] S.K. Blau, M. Visser and A. Wipf. Nucl. Phys. B310, 163 (1988).

[96] N. Balasz and C. Voros. Phys. Rep. 143, 109 (1986).

[97] M.G. Gutzwiller. Chaos in Classical and Quantum Mechanics. Springer-Verlag, New York, (1990).

[98] P. Chang and J.S. Dowker. Nucl. Phys. B407, 432 (1993).

[99] S. Minakshisundaram. J. Indian Math. Soc. 13, 41 (1949).

[100] S. Minakshisundaram. Can. J. Math. 4, 26 (1952).

[101] R. Camporesi and A. Higuchi. Phys. Rev. D47, 3339 (1993).

[102] R.S. Cahn and J. Wolf. Commun. Math. Helv. 51, 1 (1976).

[103] J.A. Wolf. Spaces of Constant Curvature. Publish or Perish, Berkely, (1977).

[104] J.S. Dowker and R. Critchley. Phys. Rev. D13, 224 (1976).

[105] A.F. Beardon. The Geometry of Discrete Groups. Springer-Verlag, Berlin, (1983).

[106] G.F.R. Ellis. Gen. Relativ. Gravit. 2, 7 (1971).

[107] I.S. Gradshteyn and I.M. Ryzhik. Table of Integrals, Series and Products. Academic Press, New York, (1980).

[108] C. G. Callan and F. Wilczek. Nucl. Phys. B340, 366 (1990).

[109] P. Candelas and S. Weinberg. Nucl. Phys. B237, 397 (1984).

[110] R. Camporesi. Comm. Math. Phys. 148, 283 (1992). 
[111] R. Camporesi and A. Higuchi. Phys. Rev. D45, 3591 (1992).

[112] B. Maskit. Kleinian Groups. Spinger-Verlag, Berlin, (1988).

[113] A. Borel. Topology 2, 111 (1963).

[114] I. Chavel. Eigenvalues in Riemannian Geometry. Academic Press, New York, (1984).

[115] M. G. G. Laidlaw and C. DeWitt-Morette. Phys. Rev. D3, 1375 (1971).

[116] D.A. Hejhal. The Selberg Trace Formula for PSL(2,R). Springer-Verlag, Berlin, (1976).

[117] I.M. Guelfand and G.E. Chilov. Les Distributions. Dunod, Paris, (1972).

[118] R. Gangolli. Illinois J. Math. 21, 1 (1977).

[119] J. Elstrod, F. Grunewald and J. Mennicke. Banach Center Publ. 17, 83 (1985).

[120] J. Elstrodt, F. Grunewald and J. Mennicke. Math. Ann. 277, 655 (1987).

[121] Y.P. Goncharov. Lett. Math. Phys. 19, 73 (1990).

[122] A.B. Venkov. Spectral theory of authomorphic functions and its applications. Kluwer Academic Publishers, Dordrecht, The Netherlands, (1990). Mathematics and Its Applications (Soviet Series) vol. 51.

[123] A.B. Venkov. Russian Math. Surveys 34, 79 (1979).

[124] M.R. Brown, A.C. Ottewill and S.T.C. Siklos. Phys. Rev. D26, 1881 (1982).

[125] C.J. Isham. Proc. R. Soc. London A362, 383 (1978).

[126] J.S. Dowker and R. Banach. J. Phys. A11, 2255 (1978).

[127] R. Banach and J.S. Dowker. J. Phys. A12, 2527 (1979).

[128] B.S. Kay. Commun. Math. Phys. 62, 55 (1978).

[129] S.A. Fulling, F.J. Narcowich and R.M. Wald. Ann. Phys. 136, 243 (1981).

[130] R. Banach and J.S. Dowker. J. Phys. A12, 2545 (1979).

[131] L.H. Ford. Phys. Rev. D14, 3304 (1976).

[132] J.S. Dowker. Class. Quantum Grav. 1, 359 (1984).

[133] A.A. Bytsenko and Y.P. Goncharov. Class. Quantum Grav. 8, 2269 (1991).

[134] A.A. Bytsenko and S. Zerbini. Class. Quantum Grav. 9, 1365 (1992).

[135] T.S. Bunch. Phys. Rev. D18, 1844 (1978).

[136] P. Candelas and J.S. Dowker. Phys. Rev. D19, 2902 (1979).

[137] A.A. Bytsenko, G. Cognola and L. Vanzo. J. Math. Phys. 33, 3108 (1992), Errata: J. Math. Phys., 34, 1614 (1993).

[138] G. Cognola, K. Kirsten and L. Vanzo. Phys. Rev. D49, 1029 (1994). 
[139] G. Cognola, Renormalization of one-loop effective action on an arbitrary curved spacetime: a general method. Phys. Rev. 50D, 909 (1994).

[140] A.L. Berkin. Phys. Rev. D46, 1551 (1992).

[141] R.J. Rivers. Path Integral Methods in Quantum Field Theory. Cambridge University Press, Cambridge, (1987).

[142] L. Ford. Phys. Rev. D21, 933 (1980).

[143] D.J. Toms. Phys. Rev. D21, 2805 (1980).

[144] G. Denardo and E. Spallucci. Nuovo Cimento A58, 243 (1980).

[145] A. Actor. Class. Quantum Grav. 7, 1463 (1990).

[146] E. Elizalde and A. Romeo. Phys. Lett. B244, 387 (1990).

[147] K. Kirsten, G. Cognola and L. Vanzo. Phys. Rev. D48, 2813 (1993).

[148] M.J. Duff. Class. Quantum Grav. 5, 189 (1988).

[149] K. Kikkawa and M. Yamasaki. Prog. Theor. Phys. 76, 1379 (1986).

[150] K. Fujikawa and J. Kubo. Phys. Lett. B199, 75 (1987).

[151] L. Mezincescu, R. Nepomechie and P. van Nieuwenhuizen. Nucl. Phys. B309, 317 (1988).

[152] U. Lindström. Int. J. Mod. Phys. A3, 2401 (1988).

[153] M.J. Duff. Class. Quantum Grav. 6, 1577 (1989).

[154] J. Barcelos-Neto and M. Ruiz-Altaba. Phys. Lett. B228, 193 (1989).

[155] R. Amorim and J. Barcelos-Neto. Int. J. Mod. Phys. A5, 2667 (1990).

[156] J. Barcelos-Neto. Phys. Lett. B245, 26 (1990).

[157] S.D. Odintsov and D.L. Wiltshire. Class. Quantum Grav. 7, 1499 (1990).

[158] P.A.M. Dirac. Proc. R. Soc. A268, 7 (1962).

[159] P.S. Howe and R.W. Tucker. J. Phis. A10, L155 (1977).

[160] M.S. Alves and J. Barcelos-Neto. Europhys. Lett. 7, 395 (1988).

[161] M. Henneaux. Phys. Lett. B120, 179 (1983).

[162] A. Strumia and G. Venturi. Lett. Nuovo Cimento 13, 337 (1975).

[163] L. Brink, P. Di Vecchia and P.S. Howe. Phys. Lett. B65, 471 (1976).

[164] A. Schild. Phys. Rev. D16, 1722 (1977).

[165] D.P. Dolan and D.H. Tchrakian. Phys. Lett. B198, 447 (1987).

[166] A.M. Polyakov. Phys. Lett. B103, 207 (1981).

[167] M.J. Bowick and S.G. Rajeev. Phys. Rev. Lett. 58, 535 (1987). 
[168] M.J. Bowick and S.G. Rajeev. Nucl. Phys. B293, 348 (1987).

[169] J. Hoppe. In Proc. Florence Workshop on Constraint Theory and Relativistic Dynamics. G. Longhi and L. Lusanna, editors. World Scientific, Singapore, (1987).

[170] B. de Wit, J. Hoppe and H. Nicolai. Nucl. Phys. B305, 545 (1988).

[171] J.M. Evans. Nucl. Phys. B298, 92 (1988).

[172] R.E. Kallosh. Phys. Lett. B195, 369 (1987).

[173] S. Carlip. Nucl. Phys. B284, 365 (1987).

[174] U. Marquand and M.Scholl. Phys. Lett. B227, 227 (1989).

[175] I. Bars. Nucl. Phys. B343, 398 (1990).

[176] C. Marshall and P. Ramond. Nucl. Phys. B85, 375 (1975).

[177] Y. Nambu. Phys. Lett. B80, 372 (1979).

[178] A.M. Polyakov. Phys. Lett. B82, 247 (1979).

[179] A.M. Polyakov. Nucl. Phys. B164, 171 (1980).

[180] T. Eguchi. Phys. Rev. Lett. 44, 126 (1980).

[181] A.T. Ogielsky. Phys. Rev. D22, 2407 (1980).

[182] J. Gamboa and M. Ruiz-Altaba. Phys. Lett. B205, 245 (1988).

[183] W. Siegel. Phys. Lett. B128, 397 (1983).

[184] J. Hughes, J. Liu and J. Polchinski. Phys. Lett. B180, 370 (1986).

[185] A. Achúcarro, J.M.Evans, P.K. Townsend and D.L. Wiltshire. Phys. Lett. B198, 441 (1987).

[186] G. Sierra. Class. Quantum Grav. 4, 227 (1987).

[187] J.A. de Azcarraga and J. Lukierski. Phys. Rev. D38, 509 (1988).

[188] M.J. Duff, T. Inami, C.N. Pope, E. Sezgin and K.S. Stelle. Nucl. Phys. B297, 515 (1988).

[189] A.A. Bytsenko and S.D. Odintsov. Class. Quantum Grav. 9, 391 (1992).

[190] A.A. Bytsenko and S.D. Odintsov. Főrtsch. der Physik 41, 1 (1993).

[191] U. Marquand, R. Kaiser and M. Scholl. Phys. Lett. B227, 234 (1989).

[192] E. Bergshoeff, E. Sezgin and P.K. Townsend. Ann. Phys. 185, 330 (1988).

[193] B. Maskit. Acta Math. 130, 243 (1973).

[194] B.N. Apanasov. Soviet Math. Dokl. 17, 1670 (1976).

[195] W.P. Thurston. Bull. Amer. Math. Soc. 6, 357 (1982).

[196] P. Scott. Bull. London Math. Soc. 15, 401 (1983). 
[197] S.L. Krushkal, B.N. Apanasov and N.A. Gusevskii. Kleinian Groups and Uniformization in Examples and Problems. Nauka (Siberian Branch), (1981).

[198] S. K. Donaldson. J. Diff. Geom. 18, 316 (1983).

[199] C.T.C. Wall. Geometries and Geometric Structures in Real Dimension 4 and Complex Dimension 2. In Lecture Notes in Mathematics. Springer Verlag, Berlin, (1986).

[200] S. Ishihara. J. Math. Soc. Japan 7, 345 (1955).

[201] A.L. Besse. Einstein Manifolds. Springer Verlag, Berlin, (1987).

[202] E. Alvarez. Some general problems in quantum gravity. In Quantum Gravity and Cosmology. J. Sola J. Perez-Mercader and E. Verdaguer, editors. World Scientific, Singapore, (1992).

[203] E. Alvarez. Int. J. Mod. Phys. D2, 1 (1993).

[204] A.A. Bytsenko and Y.P. Goncharov. Mod. Phys. Lett. A6, 669 (1991).

[205] A.A. Bytsenko and S. Zerbini. Mod. Phys. Lett. A8, 1573 (1993).

[206] P.A. Collins and R.W. Tucker. Nucl. Phys. B112, 150 (1976).

[207] E.S. Fradkin and A.A. Tseytlin. Ann. Phys. 143, 413 (1982).

[208] J. Gamboa and M. Ruiz-Altaba. Phys. Lett. B205, 145 (1988).

[209] E. D'Hoker and D.H. Phong. Commun. Math. Phys. 104, 537 (1986).

[210] P. Sarnak. Commun. Math. Phys. 110, 113 (1987).

[211] E.G. Floratos. Phys. Lett. B220, 61 (1989).

[212] E.G. Floratos and G.K. Leontaris. Phys. Lett. B223, 37 (1989).

[213] S.D. Odintsov. Europhys. Lett. 10, 439 (1989).

[214] B. Sawhill. Phys. Lett. B202, 505 (1988).

[215] A.A. Bytsenko and S. Zerbini. Nuovo Cimento A9, 1275 (1992).

[216] C.J. Isham. Proc. R. Soc. Lond. A364, 591 (1978).

[217] S.J. Avis and C.J. Isham. Nucl. Phys. B156, 441 (1979).

[218] A. Chockalingham and C.J. Isham. J. Phys. A13, 2723 (1980).

[219] I.J. Zucker. J. Phys. A7, 1568 (1974).

[220] I.J. Zucker. J. Phys. A8, 1734 (1975).

[221] E. Bergshoeff, E. Sezgin and P.K. Townsend. Ann. Phys. 185, 330 (1987).

[222] E. Bergshoeff, E. Sezgin and P.K. Townsend. Phys. Lett. B189, 75 (1987).

[223] S. Gandhi and K. S. Stelle. Class. Quantum Grav. 5, 127 (1988).

[224] E. Bergshoeff, A. Salam, E. Sezgin and Y. Tanii. Phys. Lett. B205, 237 (1988). 
[225] G.H. Hardy and S. Ramanujan. Proc. London Math. Soc. 17, 75 (1918).

[226] K. Huang and S. Weinberg. Phys. Rev. Lett. 25, 895 (1970).

[227] D. Mitchell and N. Turok. Nucl. Phys. B294, 1138 (1987).

[228] N. Matsuo. Z. Phys. C36, 289 (1987).

[229] A.A. Bytsenko, K. Kirsten and S. Zerbini. Phys. Lett. B304, 235 (1993).

[230] G. Meinardus. Math. Z. 59, 338 (1954).

[231] G. Meinardus. Math. Z. 61, 289 (1954).

[232] G.E. Andrews. The Theory of Partitions. In Encyclopedia of Mathematics and its Applications. Addison-Wesley Publishing Company, (1976).

[233] W.J. Le Veque. Reviews in Number Theory. Amer. Math. Soc., Providence, (1974).

[234] S.Fubini, A.J. Hanson and R.Jackiw. Phys. Rev. D7, 1732 (1973).

[235] D. Mitchell and N. Turok. Phys. Rev. Lett. 58, 1577 (1987).

[236] E. Alvarez and T. Ortin. Mod. Phys. Lett. A7, 2889 (1992).

[237] H.S. Green. Phys. Rev. 90, 70 (1953).

[238] Y. Ohnuki and Kamefuchi. Quantum Field Theory and Parastatistics. University of Tokyo Press, Tokyo, (1982).

[239] O.W. Greenberg and A.M.L. Messiah. Phys. Rev. 138, 1155 (1965).

[240] S. Doplicher, R. Haag and J. Roberts. Commun. Math. Phys. 23, 199 (1971).

[241] S. Doplicher, R. Haag and J. Roberts. Commun. Math. Phys. 35, 49 (1974).

[242] F. Ardalan and F. Mansouri. Phys. Rev. D9, 3341 (1974).

[243] M. Hama, M. Sawamura and H. Susuki. Prog. Theor. Phys. 88, 149 (1992).

[244] A.A. Actor. J. Phys. A20, 927 (1987).

[245] A.A. Bytsenko, S.D. Odintsov and S. Zerbini. J. Math. Phys. 2057 (1994).

[246] G. T. Horowitz and A. Strominger. Nucl. Phys. B360, 197 (1991).

[247] G.W. Gibbson and K. Maeda. Nucl. Phys. B298, 741 (1988).

[248] S.W. Hawking. Phys. Rev. D13, 191 (1976).

[249] B.Harms and Y. Leblanc. Phys. Rev. D46, 2334 (1992).

[250] B. Harms and Y. Leblanc. Phys. Rev. D47, 2438 (1993).

[251] J.D. Bekenstein. Phys. Rev. D7, 2333 (1973).

[252] S.W. Hawking. Commun. Math. Phys. 43, 199 (1975).

[253] G.'t Hooft. Nucl. Phys. B256, 727 (1985). 
[254] L. Susskind and J. Uglum. Phys. Rev. D50, 2700 (1994).

[255] J. S. Dowker. Class. Quantum Grav. 11, L55 (1994).

[256] D. V. Fursaev, Black Hole Thermodynamics and Renormalization. DSF-32/94 (1994), hepth/9408066.

[257] S. N. Solodukhin. Phys. Rev. 51, 609 (1995).

[258] G. Cognola, L. Vanzo and S. Zerbini, One-Loop quantum corrections to the entropy for a 4-dimensional eternal black hole. Preprint Trento University UTF 342 (1995), Submitted.

[259] L. Bombelli, R. Koul, J. Lee and R. Sorkin. Phys. Rev. D34, 373 (1986).

[260] M. Srednicki. Phys. Rev. Lett. 71, 666 (1993).

[261] C.G. Callan and F. Wilczek. Phys. Lett. B333, 55 (1994).

[262] D. Kabat and M. J. Strassler. Phys. Lett. B329, 46 (1994).

[263] W. Israel. Phys. Lett. A57, 107 (1976).

[264] D. W. Sciama, P. Candelas and D. Deutsch. Adv. in Physics 30, 327 (1981).

[265] J.L.F. Barbon. Phys. Rev. D50, 2712 (1994).

[266] H.E. Haber and H.A. Weldon. Phys. Rev. Lett. 46, 1497 (1981).

[267] H.E. Haber and H.A. Weldon. Phys. Rev. D25, 502 (1982).

[268] R. Camporesi. Class. Quantum Grav. 8, 529 (1991).

[269] K. Kirsten. J. Phys. A24, 3281 (1991).

[270] A.A. Bytsenko, L. Vanzo and S. Zerbini. Phys. Lett B291, 26 (1992).

[271] A.A. Bytsenko, L. Vanzo and S. Zerbini. Mod. Phys. Lett. A7, 2669 (1992).

[272] G. Cognola, K. Kirsten, L. Vanzo and S. Zerbini, Finite temperature effective potential on hyperbolic spacetimes. Phys. Rev. 49D, 5307 (1994).

[273] G. Cognola and L. Vanzo. Phys. Rev. D47, 4575 (1993).

[274] A. Einstein. Berl. Ber. 22, 261 (1924).

[275] K. Huang. Statistical Mechanics. J. Wiley and Sons, New York, (1963).

[276] H. Araki and E.J. Woods. J. Math. Phys. 4, 637 (1963).

[277] J.T. Lewis and J.V. Pulé. Commun. Math. Phys. 36, 1 (1974).

[278] L.J. Landau and I.F. Wilde. Commun. Math. Phys. 70, 43 (1979).

[279] K. Benson, J. Bernstein and S. Dodelson. Phys. Rev. D44, 2480 (1991).

[280] J. Bernstein and S. Dodelson. Phys. Rev. Lett. 66, 683 (1991).

[281] D.J. Toms. Phys. Rev. Lett. 69, 1152 (1992).

[282] L. Parker and Y. Zhang. Phys. Rev. D44, 2421 (1991). 
[283] L. Parker and Y. Zhang. Phys. Rev. D47, 416 (1993).

[284] L. Parker and Y. Zhang. Phys. Rev. D51, 2703 (1995).

[285] R. Ziff, G.E. Uhlenbeck and M. Kac. Phys. Rep. 32, 169 (1977).

[286] G. Cognola, K. Kirsten and S. Zerbini. Phys. Rev. D48, 790 (1993).

[287] R. Hagedorn. Supplemento Nuovo Cimento 3, 147 (1965).

[288] S. Fubini and G. Veneziano. Nuovo Cimento A64, 1640 (1969).

[289] R. Rhom. Nucl. Phys. B237, 553 (1984).

[290] K. Kikkawa and M. Yamasaki. Phys. Lett. B149, 357 (1984).

[291] M. Gleiser and J.C. Taylor. Phys. Lett. B164, 36 (1985).

[292] M. Bowick and L.C.R. Wijewardhana. Phys. Rev. Lett. 54, 2485 (1985).

[293] E. Alvarez. Phys. Rev. D31, 418 (1985).

[294] N. Sakai and I. Senda. Prog. Theor. Phys. 75, 692 (1986).

[295] J. Polchinski. Commun. Math. Phys. 104, 539 (1987).

[296] I. Antoniadis, J. Ellis and D.V. Nanopoulos. Phys. Lett. B199, 402 (1987).

[297] E. Alvarez and M.A.R. Osorio. Phys. Rev. 36, 1175 (1987).

[298] Y. Aharonov, F. Englert and J. Orloff. Phys. Lett. B199, 366 (1987).

[299] M. Mc Guigan. Phys. Rev. D38, 552 (1988).

[300] E. Witten. Commun. Math. Phys. 117, 353 (1988).

[301] J.J. Atick and E. Witten. Nucl. Phys. B310, 291 (1988).

[302] S.D. Odintsov. Europhys. Lett. 8, 207 (1989).

[303] N. Deo, S. Jain and C.I. Tan. Phys. Rev. D40, 2626 (1989).

[304] I. Antoniadis and C. Kounnas. Phys. Lett. B261, 369 (1991).

[305] S.D. Odintsov. Rivista Nuovo Cim. 15, 1 (1992).

[306] R. Brandenberger and C. Vafa. Nucl. Phys. B316, 391 (1989).

[307] E. Alvarez. Nucl. Phys. B269, 596 (1986).

[308] J. Polchinski. Phys. Rev. Lett. 68, 1267 (1992).

[309] M.B. Green. Phys. Lett. B282, 380 (1992).

[310] S.D. Odintsov. Phys. Lett. B252, 573 (1990).

[311] A.A. Bytsenko, E. Elizalde, S.D. Odintsov and S. Zerbini. Nucl. Phys. B394, 423 (1993).

[312] A.A. Bytsenko, E. Elizalde, S.D. Odintsov and S. Zerbini. Mod. Phys. Lett A8, 1131 (1993). 
[313] B. Mc Clain and B.D.B. Roth. Commun. Math. Phys. 111, 539 (1987).

[314] P. Murphy and S. Sen. Phys. Lett. B233, 322 (1989).

[315] E. Alvarez and T. Ortin. Phys. Lett. B241, 215 (1990).

[316] A.A. Bytsenko, E. Elizalde, S.D. Odintsov and S.Zerbini. Phys. Lett. B311, 87 (1993).

[317] E. D’Hoker and D.H. Phong. Rev. Mod. Phys. 60, 917 (1988).

[318] W. Lerche, A.N. Schellekens and N.P. Warner. Phys. Rep. 177, 1 (1989).

[319] Staff of the Bateman Manuscript Project. Higher Trascendental Functions. In A. Erderlyi. McGraw-Hill Book Company, New York, (1955). Based on the notes of Harry Bateman. 